\title{
Report Title: An Integrated Approach to Modeling And Mitigating SOFC Failure
}

Type of Report: Final Technical Report

Reporting Period Start Date: October 1, 2002

Reporting Period End Date: May 15, 2006

Principal Author(s): Jianmin Qu, Andrei Fedorov, and Comas Haynes

Date Report was Issued: July 1, 2006

DOE Award Number: No. DE-FC26-02NT41571

Name and Address of Submitting Organization: Georgia Tech, Atlanta, GA 30332-

0405

DISCLAIMER* This report was prepared as an account of work sponsored by an agency of the United States Government. Neither the United States Government nor any agency thereof, nor any of their employees, makes any warranty, express or implied, or assumes any legal liability or responsibility for the accuracy, completeness, or usefulness of any information, apparatus, product, or process disclosed, or represents that its use would not infringe privately owned rights. Reference herein to any specific commercial product, process, or service by trade name, trademark, manufacturer, or otherwise does not necessarily constitute or imply its endorsement, recommendation, or favoring by the United States Government or any agency thereof. The views and opinions of authors expressed herein do not necessarily state or reflect those of the United States Government or any agency thereof. 


\begin{abstract}
The specific objectives of this project were:

(1) To develop and demonstrate the feasibility of an integrated predictive computerbased tool for fuel cell design and reliability/durability analysis,

(2) To generate new scientific and engineering knowledge to better enable SECA Industry Teams to develop reliable, low-cost solid-oxide fuel cell power generation systems,

(3) To create technology breakthroughs to address technical risks and barriers that currently limit achievement of the SECA performance and cost goals for solidoxide fuel cell systems, and

(4) To transfer new science and technology developed in the project to the SECA Industry Teams.

Through this three-year project, the Georgia Tech's team has demonstrated the feasibility of the solution proposed and the merits of the scientific path of inquiry, and has developed the technology to a sufficient level such that it can be utilized by the SECA Industry Teams. This report summarizes the project's results and achievements.
\end{abstract}




\section{TABLE OF CONTENTS}

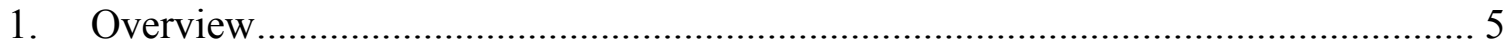

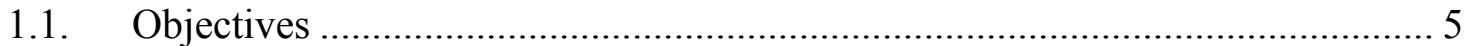

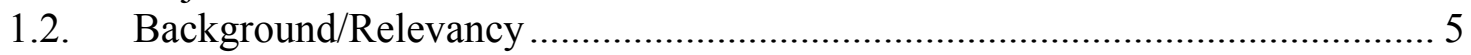

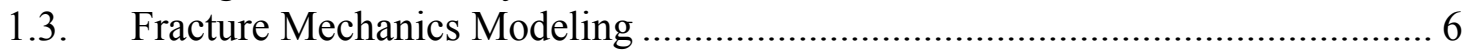

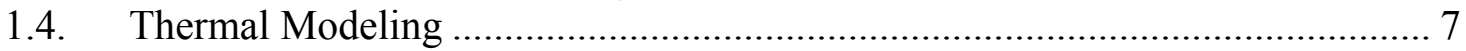

2. Fracture Mechanics Modeling …………………............................................. 8

2.1. Energy release rates for edge delaminations and buckling .............................. 8

2.1.1. Edge Delamination Crack ............................................................ 9

2.1.2. Blister Delamination ........................................................................ 10

2.1.3. Criteria for estimating maximum tolerable fabrication defects in SOFCs 13

2.2. Micromechanics Modeling of Porous Anode ………..................................... 14

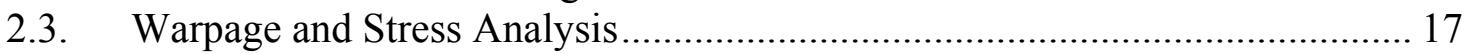

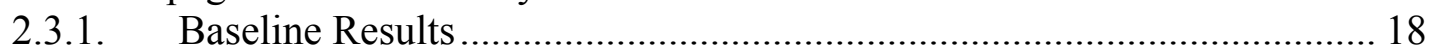

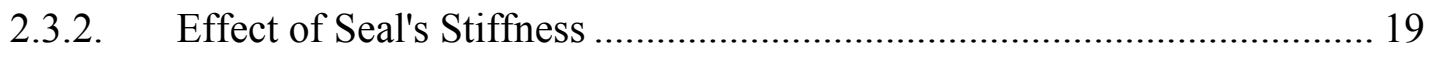

2.3.3. Effect of Nonuniform Anode Porosity.................................................... 21

2.4. Spalling Phenomenon during Thermal Transients and Shock......................... 23

2.5. 2D Finite Element Analysis of Crack Path Selection and Propagation............ 25

2.5.1. Crack Growth within Anode................................................................ 26

2.5.2. Behavior of Edge Delamination............................................................. 28

2.6. Fracture Mechanical Analyzer (FMA) - Theory and Background................... 30

2.6.1. Fracture Mechanics....................................................................... 31

2.6.2. Domain Integral (Energy Release Rate ) ................................................... 33

2.6.3. Thermal Domain Integral......................................................................... 36

2.6.4. Interaction Integral (Stress Intensity Factors)............................................. 36

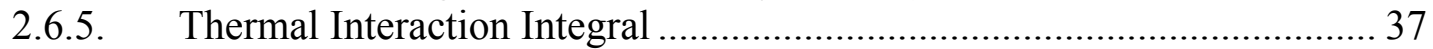

2.6.6. Finite Element Implementation........................................................... 38

2.6.7. Finite Element Calculations ..................................................................... 39

2.6.8. Program Summary .................................................................................. 41

2.7. FMA Verification: Axisymmetric Bimaterial and Thermally Stressed Cracks 42

2.7.1. Bimaterial Penny Shaped Crack ........................................................... 42

2.7.2. Thermally Stressed Straight Crack …………............................................ 46

2.7.3. FMA using MARC FEM Commercial Software...................................... 48

2.8. Creep Prediction in Ni/YSZ Cermet Anodes ................................................ 52

2.8.1. Analytical Creep Model for Ni/YSZ Cermet Anodes............................... 52

2.8.2. FEM creep models for reduced Ni/YSZ anodes ...................................... 58

2.9. First Order Failure Criteria for SOFC PEN Structure ....................................... 61

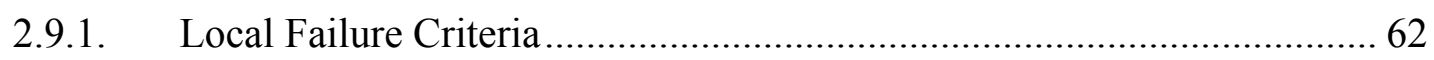

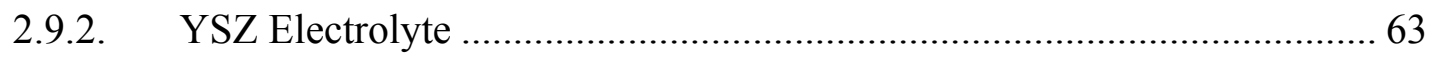

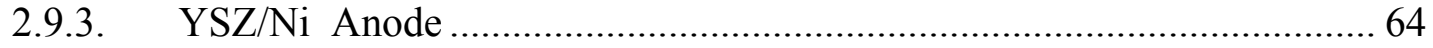

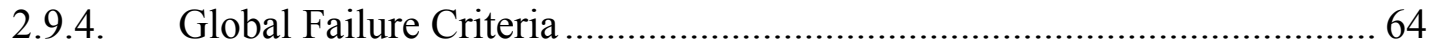

2.10. Thermal Shock induced Failure of Anode Materials ................................... 70

2.11. Develop models for thermal shock-induced failure in SOFC ....................... 77

2.12. Develop models for thermal shock-induced failure in SOFC ....................... 82

3. Thermal Modeling ............................................................................................ 91

3.1. Importance of Thermal Radiation Heat Transfer................................................ 91 
3.2. Two-flux Approximation for Numerical Calculation of Radiative Heat Transfer 92

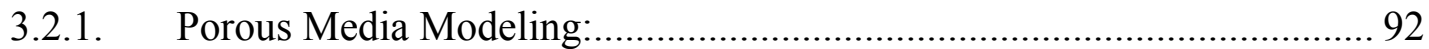

3.3. Engineering Code to Compare Performance and Reliability Trends................ 94

3.4. Theoretical Methodology for Modeling Gas flow, Mass, and Heat transfer.... 97

3.4.1. Binary Mass Diffusion:........................................................................ 97

3.4.2. Knudsen Diffusion:............................................................................ 99

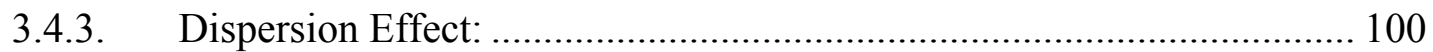

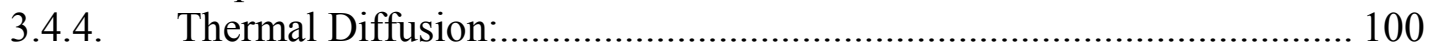

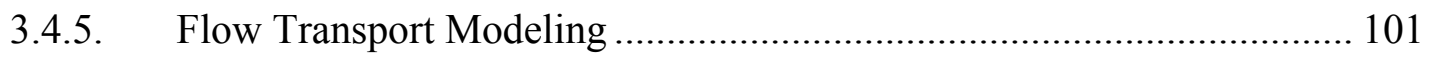

3.5. Summary of Radiative Properties Evaluation................................................. 101

3.5.1. Technical Description of the Experimental Work .................................. 102

3.6. Code validation for solution of radiative heat flux ......................................... 103

3.6.1. Implementation of 2-flux method in SOFC model ................................ 105

3.6.2. Concluding Remarks........................................................................... 106

3.7. Constitutive Models for Applications of the Two-temperature Non-equilibrium

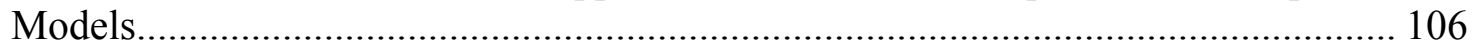

3.7.1. Formulation of first-order transient heating model................................ 107

3.7.2. Further Development Formulation of first-order transient heating model 110

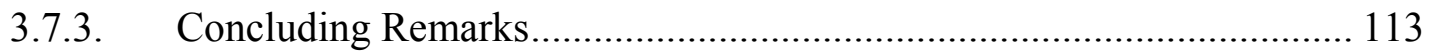

3.8. Second-order transient heating model ........................................................ 113

3.8.1. Formulation and solution of second-order transient heating model ....... 113

3.8.2. Results of second-order transient heating model .................................... 116

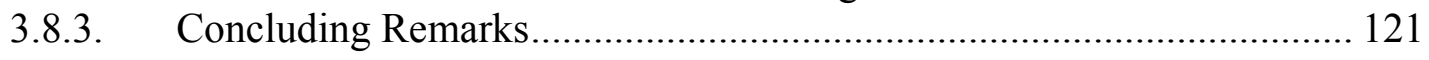

3.9. Formulation and Analysis of CFD model ..................................................... 121

3.9.1. Temperature Gradient Field............................................................ 125

3.10. Simplification of $3^{\text {rd }}$ Order Model: 2-eqn coupled solid-gas model ........... 126

3.10.1. Validation 2-eqn coupled solid-gas model ............................................ 128

3.10.2. Concluding Remarks....................................................................... 130

3.10.3. Two-Equation Coupled Solid-Gas Model ............................................ 130

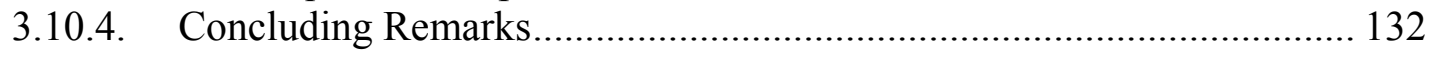

3.11. Evaluation of transient thermal models ................................................. 132

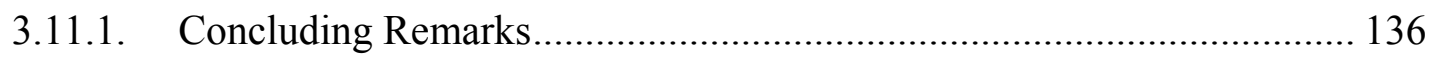

REFERENCES ............................................................. Error! Bookmark not defined.

Appendix A: Thermal Residue Stress Induced Deformation in Functionally Graded

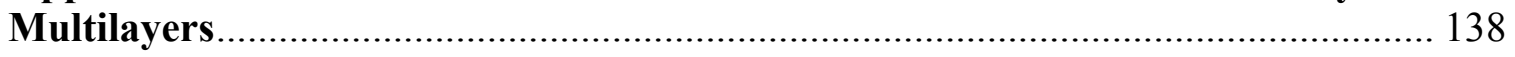

Appendix B: Thermal Shock Induced Microcrack Initiation ................................ 142

Appendix C: Expressions of $\boldsymbol{Y}$ for Various Crack Configurations .......................... 146

Appendix D: Schuster-Schwartzchild Two-Flux Approximation ........................... 149

Appendix E: 1D Derivation of Transient Solution …………………................. 152

Appendix F：2D Transient Derivation ............................................................ 153 


\section{Overview}

\subsection{Objectives}

The Solid State Energy Conversion Alliance (SECA) Core Technology Program consisted of two phases and had the following main objectives:

(1) Generate new scientific and engineering knowledge to better enable SECA Industry Teams to develop low-cost solid-oxide fuel cell power generation systems.

(2) Create technology breakthroughs to address technical risks and barriers that currently limit achievement of the SECA performance and cost goals for solid-oxide fuel cell systems.

(3) Transfer new science and technology developed in the Core Technology Program to the SECA Industry Teams.

Phase I of the Core Technology Program was a one-year effort to investigate and evaluate the feasibility of the solution proposed and/or the merits of the scientific path of inquiry. Phase II projects sought to mature the science and technology developed to a sufficient level, such that the SECA Industry Teams can utilize it.

The specific objective of this project was to develop and demonstrate the feasibility of an integrated, predictive computer-based tool for fuel cell design and reliability/ durability analysis.

\subsection{Background/Relevancy}

In SOFCs, delamination occurs due to differing thermal expansion coefficients between composite layers (i.e., electrodes and electrolyte), and more importantly, between cell and adjoining stack structure. Rapid thermal cycling causes thermal shock issues (e.g., within interconnect interfaces with electrodes, as well as within the bulk support anode.). Finally, excessive temperature gradients along the cell tends to create warpage, which when confined results in stresses. Previous modeling attempts to characterize SOFC failure modes have been limited because of simplified transport models. One dimensional, "bulk" electrochemical and thermal models have been applied, and these models have been decoupled (i.e., interdependency between electrochemistry and thermal transport neglected). In order to create greater fidelity within thermomechanical failure analysis models, interdependency between structural issues and electrochemical/thermal transport phenomena must be characterized. This requires a multi-physics modeling approach as proposed in this project.

It is well known that thermal transients and gradients impose states of stress within SOFC cell materials that may result in crack initiation, propagation and subsequent structural failure or performance degradation in short-term operation; furthermore, due to a variety of mechanisms, performance may significantly decrease with operation time. The commercial viability of SOFC power generation systems is dependent upon making significant progress in the durability and reliability of cell and stack structures. However, no systematic study of the causes or physical drivers of cell failure and degradation have been conducted; as a result, no coherent degradation or useful life prediction modeling methodology is currently available. This project took an integrated approach to modeling and mitigating SOFC failure based upon utilizing micro- 
, meso-, and macro-characterization of SOFC cell constituent layers and stacks in order to reduce the problem of performance evolution down to the evolution of material states and stress states as a function of operating time, cell/stack design and thermal/electrochemical conditions. The material states include changes in physical, mechanical, chemical and thermal properties. This modeling methodology will significantly contribute to SECA SOFC system performance requirements.

\subsection{Fracture Mechanics Modeling}

- Energy release rates for both the edge delamination and buckling driven blister delamination were obtained. Developed criteria for estimating maximum tolerable fabrication defects in SOFCs. The criteria were based on fracture analysis and formulated in a graphic map that can be conveniently used by the industry.

- Developed an analytical formula to predict the properties of porous media such as the anode and cathode. Such an analytical tool can be used to conduct parametric studies to investigate the effects of a material's microstructure on the overall thermomechanical stresses and reliability. Another importance of this analytical tool is that it can reduce significantly the amount of experimental tests to characterize the materials.

- Developed a computational algorithm to analyze the effects of different seal stiffnesses upon cell stresses and deformation.

- Developed a model for the spalling phenomenon and thermal expansion induced stress during thermal transients and shock. The model relates the rate of heat generation in the cell to microcrack initiation. Once the fracture toughness of the electrodes are known, this model can be used predict the maximum allowable heat generation before microcracks are initiated.

- Developed methods for accurately calculating the stress intensity factors and the energy release rate have been developed for a SOFC cell model. It was found that a crack beyond a critical size in the anode will lead to unstable fracture of electrolyte, which may result in fuel leakage. Edge delamination crack, on the other hand, may not be as catastrophic, for such crack will most likely to remain on the anode/electrolyte interface.

- Develop the Fracture Mechanics Analyzer software for cracking failure analysis in SOFCs, based on a domain integration formulation to evaluate crack tip parameters for fracture analysis under non-uniform temperatures. Such computation was not possible using existing commercial software.

- Provided examples of FMA program; including two verification examples and implementation into MARC.

- Developed a set of first order failure criteria for the initial design and optimization of SOFCs.

- Develop a statistic approach to predict thermal shock induced failure of anode materials and implemented this approach into MARC. 
- Developed a computational algorithm to obtain optimized values of the heating rate and air flow velocity for fast start-up without thermal shock induced fracture.

\subsection{Thermal Modeling}

- Identified an importance of thermal radiation heat transfer to accurate prediction of the local temperature field along the SOFC. Found that neglecting thermal radiation heat fluxes results in as much as $150^{\circ} \mathrm{C}$ overprediction of the cell temperature as well as much steeper axial temperature gradients along the cell.

- Developed and validated a simplified two-flux approximation for numerical calculation of radiative heat transfer in the case of gray and optically thin electrolyte of SOFCs. The model was implemented as a subroutine module that is readily integrated within the NETL computational platform for SOFC analysis. Usage of the two-flux approximation allowed for a ten-fold reduction in the required computational time as compared to the standard discrete ordinate method for treating the radiative heat transfer.

- Finished the pilot demonstration of an engineering code, graphical user interface that allows for SECA industry teams to dynamically and interactively compare performance and reliability trends associated with pertinent process design variables. The tool was very well received by industry partners (i.e., at the Sacramento SECA CTP Review) as a viable aid to their design efforts.

- Developed an advanced theoretical methodology for modeling gas flow, mass, and heat transfer in the porous electrodes of SOFCs. It accounts for local thermal non-equilibrium between the porous solid matrix and the flowing gas, the thermal-diffusion and dispersion effects in mass transport, and the effective thermophysical properties of the random porous media of electrodes.

- It was experimentally determined using FTIR spectrometer that the electrode (anode made of $40 \mathrm{vol} \% \mathrm{Ni} ; 60 \mathrm{vol} \%$ 8YSZ and cathode made of Sr-doped Lanthanum ferrite) samples appear to be opaque over the entire near and mid infrared spectra.

- Extended the two-flux solution method for radiative heat transfer in the optically thin electrolyte of the planer SOFC from the gray basis to comprehensive spectral calculations based on measured optical properties of the anode material, and wrote and validated code for implementation of the formulation. Using spectral calculations determined the limiting thickness of the electrolyte layer when radiative effects become significant and need to be accounted for.

- Theoretically demonstrated that a single temperature (local equilibrium) thermal model is sufficiently accurate for predicting the steady state temperature fields in the SOFC porous electrodes. Developed the criteria and constitutive models for 
applications of the two-temperature non-equilibrium models in modeling heat transfer in SOFC electrodes.

- Developed the first-order analytical model of the SOFC heating/cooling during start-up/shut-down in the limit of optically thin PEN assembly and local thermal equilibrium between the layers. Established the design map for safe operation based on the operating conditions and allowed transient uptake. The design map allows one to predict the required rate of air temperature increase at the inlet of the cell that would result in the optimal (minimum) start-up time without thermomechanical failure due to excessive spatial and temporal temperature gradients within the cell layers.

- Developed higher order (second and third) analytical model of the SOFC heating/cooling during start-up/shut-down in the limit of optically thin PEN assembly and local thermal equilibrium between the layers.

- Develop and implement transient, 3-D, CFD model for validation of 1-D, simplified analytical and numerical models.

- Develop and implement $3^{\text {rd }}$ order, coupled numerical model for transient heating of the unit cell. Validate and analyze the $3^{\text {rd }}$ order, coupled numerical model for transient heating of the unit cell and comparison to simplified analytical models and 3-D Fluent model.

- Evaluation of transient thermal models and selection of model that best balances accuracy, simplicity, and computational efficiency.

\section{Fracture Mechanics Modeling}

\subsection{Energy release rates for edge delaminations and buckling}

During this section, two kinds of cracks were considered, an edge delamination crack as shown in Figure 2-1 and a blister crack as shown in Figure 2-2. The anode-supported SOFCs are generally fabricated by co-firing the electrolyte/anode bilayer at 1400 $1500^{\circ} \mathrm{C}$. Because of the thermal mismatch between the electrolyte (YSZ) and the anode $(\mathrm{Ni}+\mathrm{YSZ})$, a compressive residual stress is developed in the electrolyte layer after sintering. Such thermal residual stress may cause the electrolyte layer to delaminate from the anode.

A commonly used parameter to quantify the potential delamination is the interfacial crack energy release rate, which is defined as the amount of energy released per unit crack growth. The energy release rate is an indication of the driving force for crack growth. A delamination initiation criterion is

$$
G \geq G_{i c}
$$

where $G$ is the energy release rate and $G_{i c}$ is the interfacial fracture toughness of the electrolyte/anode interface. The value of $G_{i c}$ must be measured experimentally. In what follows, the energy release rate $G$ is computed for the two kinds of cracks shown in 
Figure 2-1 and Figure 2-2. The material properties used in all calculations are listed in Table 2-1: Material Properties.

Table 2-1: Material Properties.

\begin{tabular}{|l|l|l|l|}
\hline Materials & $\begin{array}{l}\text { Young's } \\
\text { modulus }(\mathrm{GPa})\end{array}$ & Poisson's ratio & $\mathrm{CTE}\left(10-6 /{ }^{\circ} \mathrm{C}\right)$ \\
\hline Cathode $(\mathrm{LSM}+\mathrm{YSZ})$ & 90 & 0.3 & 10.56 \\
\hline Electrolyte $(\mathrm{YSZ})$ & 200 & 0.3 & 10.56 \\
\hline Anode $(\mathrm{Ni}+\mathrm{YSZ})$ & 96 & 0.3 & 12.22 \\
\hline
\end{tabular}

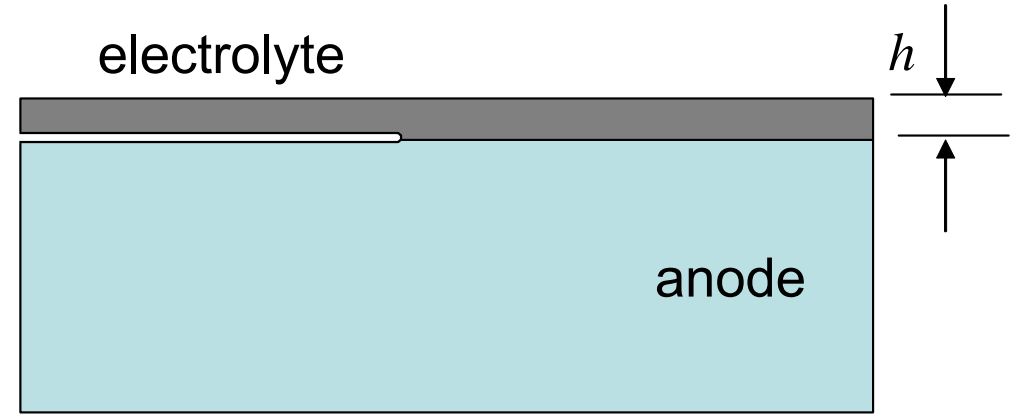

Figure 2-1: Edge Delamination Crack

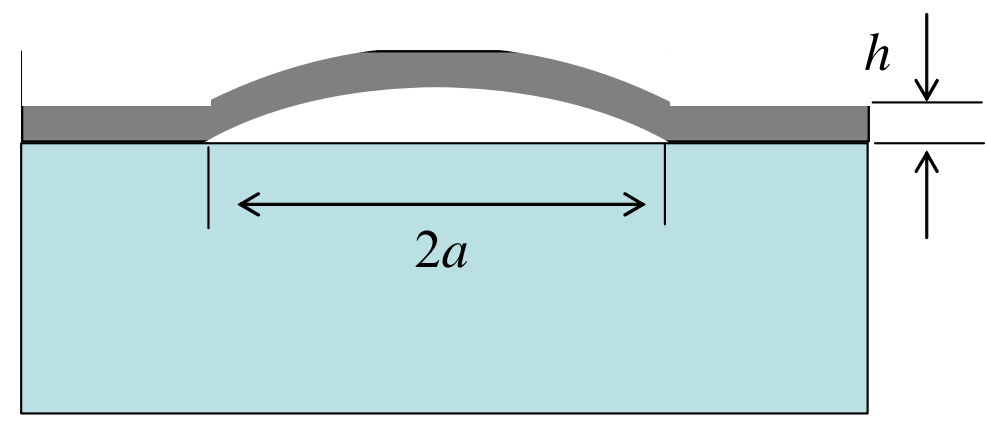

Figure 2-2: Blister Crack

\subsubsection{Edge Delamination Crack}

Based on elasticity theories, the biaxial compressive stress in the electrolyte layer is given by

$$
\sigma=\frac{E}{1-v} \Delta T \Delta \alpha
$$

where $\mathrm{E}$ and $v$ are, respectively, the Young's modulus and Poisson's ratio of the electrolyte, $\Delta T$ is the temperature differential (in our case, it is the different between processing and room temperature), $\Delta \alpha$ is the difference in the coefficient of thermal expansion between the electrolyte and anode materials. It follows from Table 2-1 that

$$
\Delta \alpha=1.66 \times 10^{-6} /{ }^{0} \mathrm{C}
$$


For processing temperature around $1400^{\circ} \mathrm{C}$, the resulting thermal residual stress, based on (2.2), is $664 \mathrm{MPa}$. This is consistent with the experimental values reported in the literature.

The elastic strain energy stored in the electrolyte layer per unit area is

$$
\frac{1-v}{E} \sigma^{2} h
$$

where $\mathrm{h}$ is the electrolyte layer thickness. Since the portion of already delaminated electrolyte layer is stress free, one may say that all the strain energy stored in that portion of the layer has been released due to the edge delamination. Therefore, the energy release rate due to per unit delamination growth should be equal to the strain energy stored in the layer per unit area, i.e.,

$$
G=1.028 \frac{1-v}{E} \sigma^{2} h
$$

where the factor 1.028 is to account for the biaxial stress effect. Substitution of (2.2) into (2.5) yields the energy release rate of the edge delamination when subjected to a temperature change of $\Delta T$,

$$
G=1.028 \frac{E h}{1-v}(\Delta T \Delta \alpha)^{2} .
$$

Clearly, for a given material system and given temperature change, the driving force for delamination is proportional to the electrolyte layer thickness. Thus, thicker electrolyte layers have higher propensity for edge delamination. The critical thickness for delamination is derived from (2.6) by making use of the fracture criterion (2.1),

$$
h_{c}=\frac{G_{i c}(1-v)}{1.028 E(\Delta T \Delta \alpha)^{2}} .
$$

where $G_{i c}$ is the interfacial fracture toughness, which must be measured experimentally.

Now consider a typical case, where the electrolyte layer thickness is $h=20 \mu \mathrm{m}$, and the processing temperature change is $1400^{\circ} \mathrm{C}$. The corresponding energy release rate for an edge delamination is (from (2.6)) $G=31.73 \mathrm{~J} / \mathrm{m}^{2}$. This must be compared with the measured interfacial fracture toughness to assess the tendency for delamination.

\subsubsection{Blister Delamination}

After processing, defects (flaws) may exist along the electrolyte/anode interface. When the flaw size is sufficiently small, local stress fields near the defect are not affected because the electrolyte layer above the flaw remains flat as shown in Figure 2-3. 


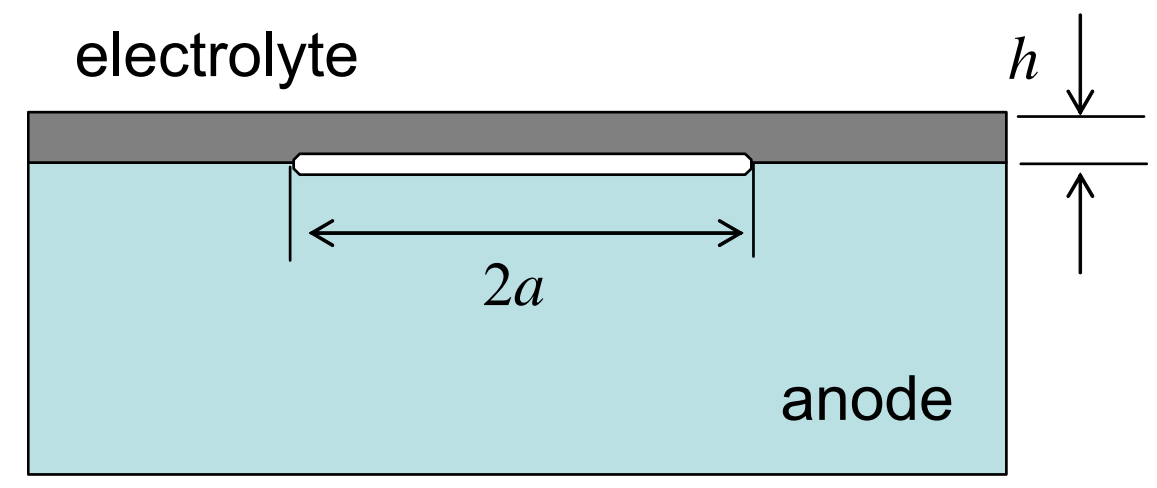

Figure 2-3: A small interfacial flaw

When the flaw size increases, it may reach a critical length such that the electrolyte layer above the flaw buckles under compressive stress after sintering, as shown in Figure 2-2. The buckled electrolyte layer generates significant stresses near the edge of the flaw, which may induce further interfacial fracture.

The following analysis outlines methods to estimate the critical flaw size for buckling and to calculate the energy release rate for interfacial fracture once buckling occurred.

First, consider the critical flaw size for buckling. Assume the initial flaw is a circular delamination of radius $a$ between the electrolyte layer of thickness $h$ and a thick anode substrate. In the analysis, the anode layer is treated as a semi-infinite space in order to simplify the algebra. This assumption introduces very little error for anodesupported cells because the thickness of the anode is orders of magnitude larger.

Based on the von Karman nonlinear plate theory, the critical in-plate compressive stress for buckling of a circular plate of radius $a$ is

$$
\sigma_{c}=1.2235 \frac{E}{1-v^{2}}\left(\frac{h}{a}\right)^{2}
$$

where it is assumed that the edge of the circular plate is clamped. By equating (2.2) and (2.8), we obtain an equation that relates the critical flaw size $a_{c}$ to the processing temperature and the electrolyte layer thickness $h$,

$$
\Delta T \Delta \alpha=1.2235 \frac{1}{1+v}\left(\frac{h}{a}\right)^{2} .
$$

This relationship is graphically shown in Figure 2-4 for the materials considered here, where the relationship between the processing temperature and the corresponding compressive stress in the electrolyte layer is also shown between the bottom and top horizontal axis. For example, $1400^{\circ} \mathrm{C}$ processing temperature corresponds to $\sim 660 \mathrm{MPa}$ of compressive stress. It is seen from Figure 2-4 that the cells are fairly insensible to processing induced initial flaws. For an electrolyte layer of thickness $20 \mu \mathrm{m}$, it can tolerate initial flaws of up to about $700 \mu \mathrm{m}$ in diameter. This seems to indicate that 
buckling driven (blister) delamination is unlikely in SOFCs, or at least it may not be a dominant failure mode. However, as the technology progresses, electrolyte as thin as 5 $\mu \mathrm{m}$ is becoming common. In such cases, blister may become a prominent failure mode since the allowable size is now only about $250 \mu \mathrm{m}$ at $900^{\circ} \mathrm{C}$.

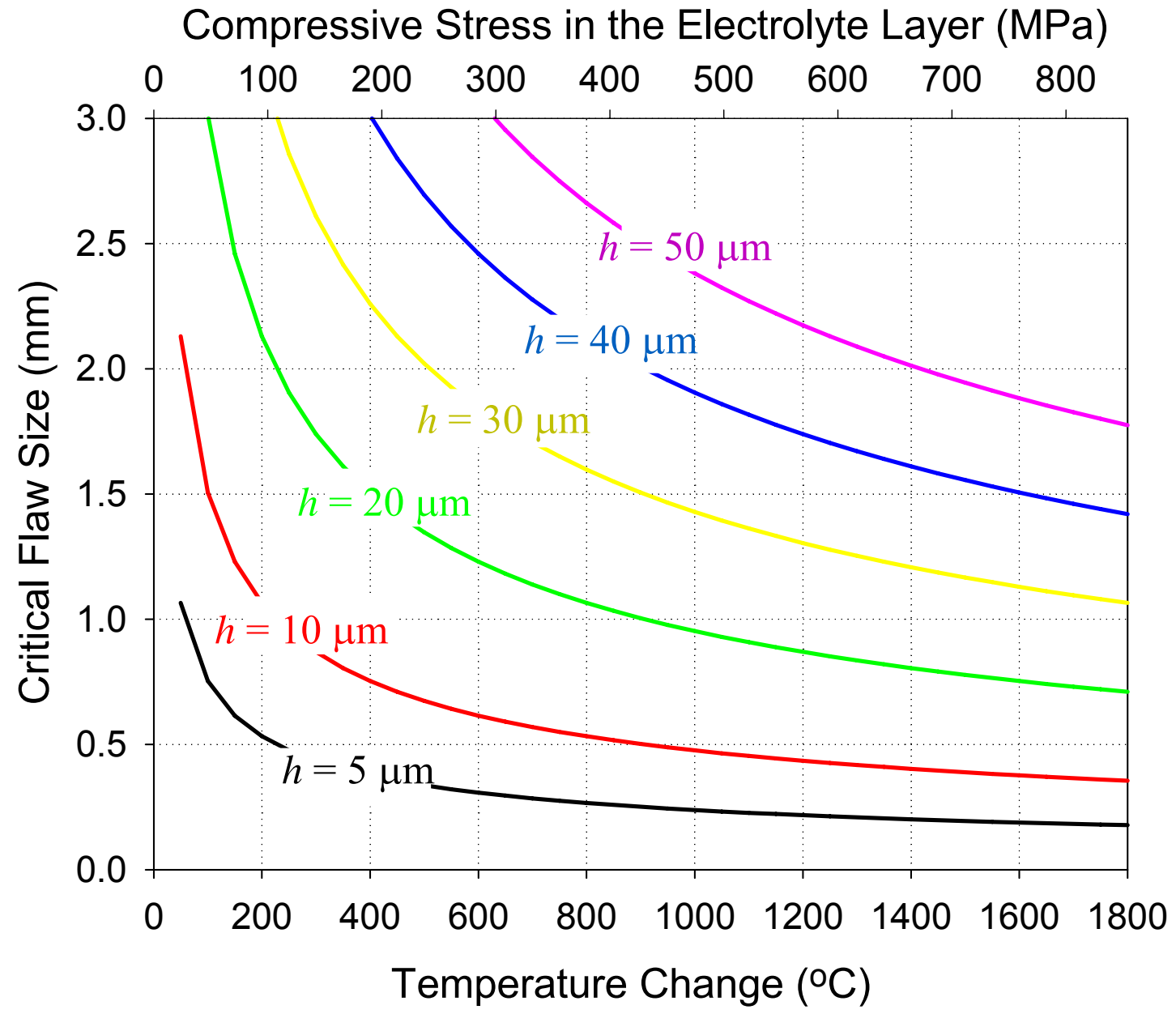

Figure 2-4: Relationships between processing temperature, electrolyte layer thickness and critical flaw size.

Next consider the energy release rate. For the circular blister delamination, the energy release rate is given by

$$
G=\frac{(\Delta T \Delta \alpha)^{2} E h}{(1-v)[1+0.9021(1-v)]}\left\{1-\left[\frac{1.2235}{\Delta T \Delta \alpha(1+v)}\left(\frac{h}{a}\right)^{2}\right]^{2}\right\}
$$

This equation gives the relationship among four important parameters, the energy release rate $(\mathrm{G})$, the processing temperature $(\Delta T)$, the electrolyte layer thickness $(h)$, and the size of the blister delamination $(2 a)$.

For a typical sintering temperature, $\Delta T=1400^{\circ} \mathrm{C}$, the energy release rate of a circular blister delamination as a function of the electrolyte layer thickness is plotted in Figure 2-5 for various initial flaw sizes. It is seen that even for an initial flaw as large as 
$0.5 \mathrm{~mm}$, the energy release rate for blister delamination is practically zero if the electrolyte layer thickness is greater than $25 \mu \mathrm{m}$. This is because, for this electrolyte layer thickness, no buckling occurs. Also shown in Figure 2-5 in dashed line is the energy release rate for an edge delamination crack. It is seen that for the same electrolyte layer thickness, the driving force for delamination is higher for the edge than the blister crack.

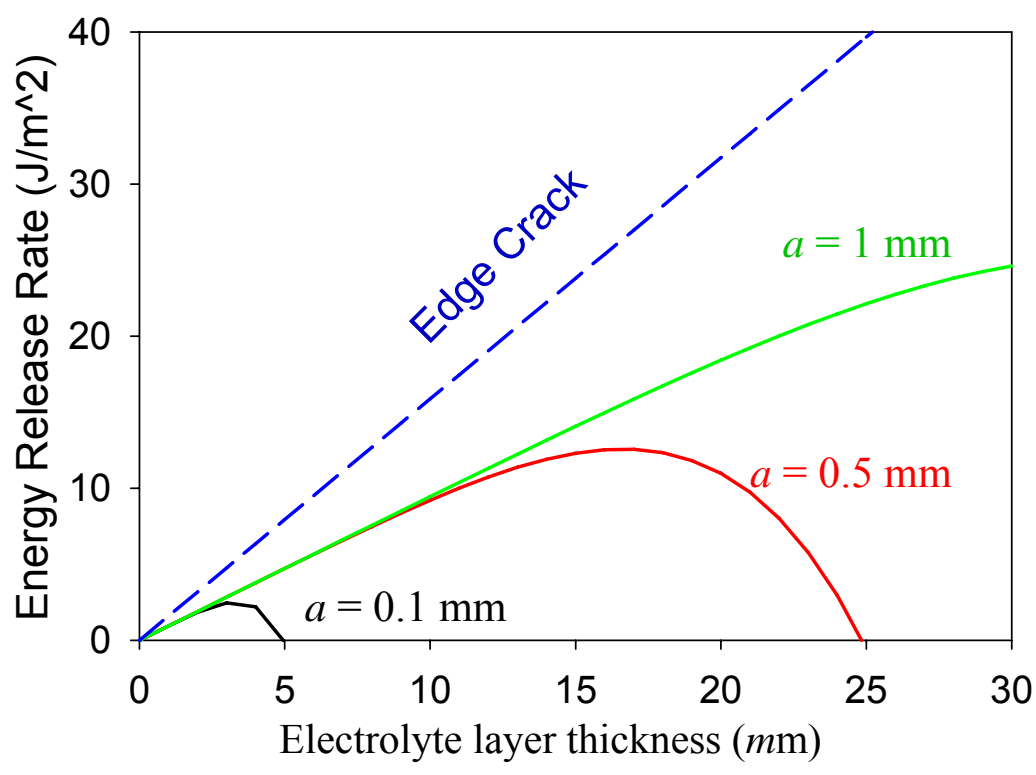

Figure 2-5: Energy release rate of a circular blister delamination vs. electrolyte layer thickness for various initial flaw sizes; the dashed line is for the energy release rate of an edge delamination. The processing temperature is set at $\Delta T=1400^{\circ} \mathrm{C}$

\subsubsection{Criteria for estimating maximum tolerable fabrication defects in SOFCs}

To further investigate the propensity of edge delamination vs. blister delamination, the energy release rate for both failure modes are plotted in Figure 2-6 as a function of initial flaw size for various electrolyte layer thickness. The solid lines are for blister delamination and the dashed lines are for the edge delamination which is independent of the initial flaw size. It is seen that the energy release rate (driving force for fracture) for edge delamination is much higher than that for blister delamination. This further underlines the earlier conclusion that blister delamination is not very likely, or at least is not a dominant failure mode. 


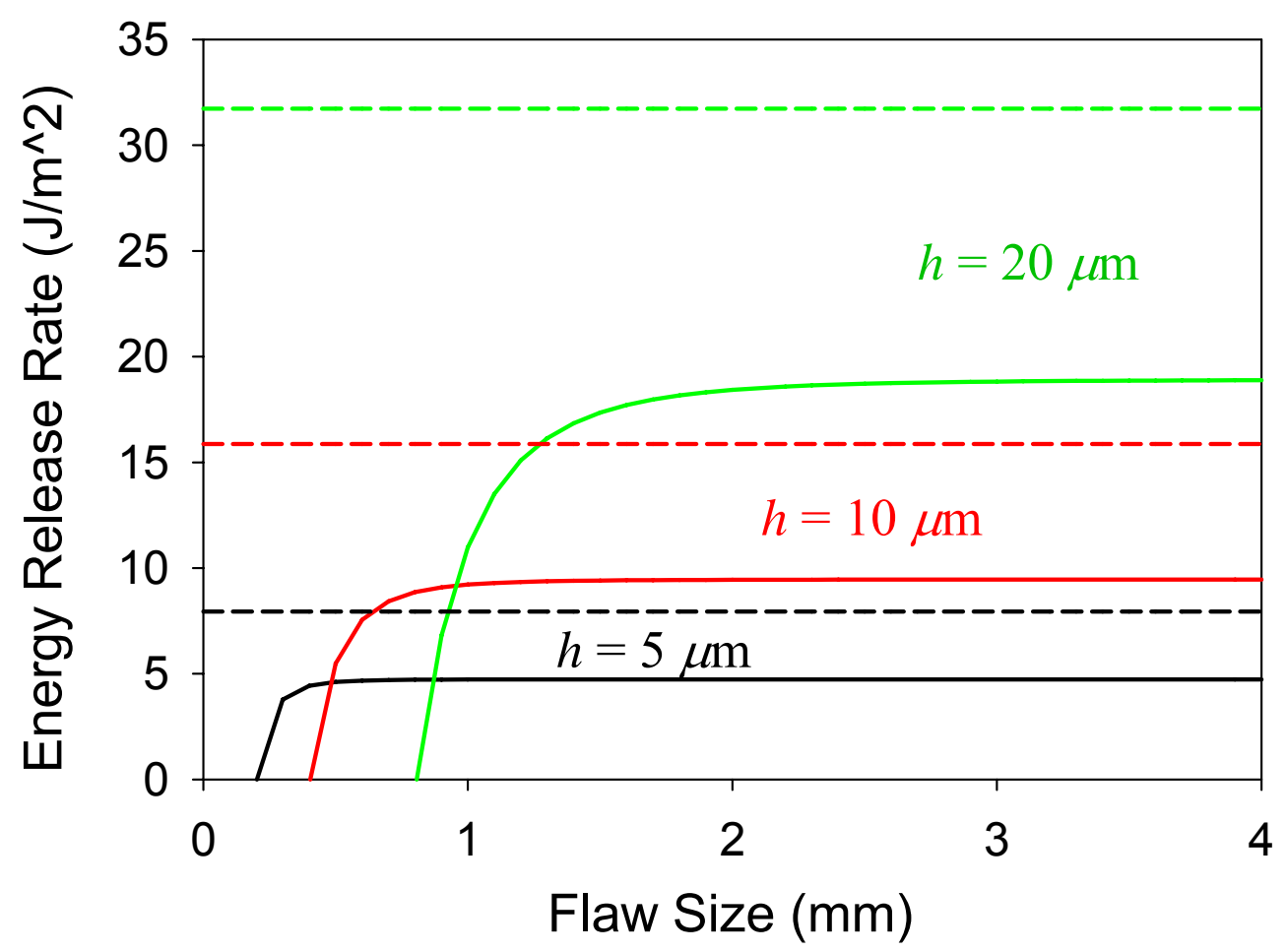

Figure 2-6: Comparison of energy release rate between edge and blister delamination

\subsection{Micromechanics Modeling of Porous Anode}

The Ni/YSZ cermet can be modeled as a porous medium with spherical voids. Its effective Young's modulus and Poisson's ratio were computed based on the Mori-Tanaka method in micromechanics. The predicted results are in good agreement with the experimental data from ORNL. The advantage of this methodology for predicting the effective properties is that one can estimate the graded properties through the thickness of the anode layer when porosity changes. The formulas developed require no fitting parameters. The input to the formulas is the properties of $\mathrm{YSZ}$ and $\mathrm{Ni}$, both are fairly well characterized. In other words, once the properties of Ni and YSZ are known, the properties of the Ni/YSZ cermet can be computed using an analytical expression for various porosity levels. In the following, we will present the results. For detailed derivation, please see the reference [Qu, J., "Effects of Slightly Weakened Interfaces on the Overall Elastic Properties of Composite Materials," Mechanics of Materials, 14, p.269 - 281, 1993]

Let the Young's modulus and the Poisson's ratio for YSZ and Ni be, respectively, $E_{Y}$, $v_{Y}$, and $E_{N}, v_{N}$. Based on the Mori-Tanaka method, the Young's modulus and Poisson's ratio of the porous $\mathrm{Ni} / \mathrm{YSZ}$ anode are given by

$$
E=\frac{2(1-c)\left(5 v_{0}-7\right) E_{0}}{2\left(5 v_{0}-7\right)+c\left(13-2 v_{0}-15 v_{0}^{2}\right)}
$$




$$
v=\frac{2\left(10 v_{0}^{2}-9 v_{0}-7\right)+c\left(25 v_{0}^{2}+6 v_{0}-19\right)}{2\left(5 v_{0}-7\right)+c\left(15 v_{0}^{2}+2 v_{0}-13\right)}
$$

where $c$ is the porosity (volume \%), and

$$
E_{0}=\frac{9 K_{0}}{1+3 K_{0} / \mu_{0}} \quad, \quad v_{0}=\frac{1-2 \mu_{0} / 3 K_{0}}{2+2 \mu_{0} / 3 K_{0}}
$$

are, respectively, the Young's modulus and Poisson's ratio of the fully densified Ni/YSZ mixture. Assume the volume fraction of $\mathrm{Ni}$ in the fully densified Ni/YSZ mixture is $c_{N}$. Then, based on the Mori-Tanaka method, we have,

$$
\begin{aligned}
& \mu_{0}=\mu_{Y}\left\{1+\frac{c_{N}\left(\mu_{N}-\mu_{Y}\right)}{\mu_{Y}+2 \delta\left(1-c_{N}\right)\left(\mu_{N}-\mu_{Y}\right)}\right\} \\
& K_{0}=K_{Y}\left\{1+\frac{c_{N}\left(K_{N}-K_{Y}\right)}{K_{Y}+3 \gamma\left(1-c_{N}\right)\left(K_{N}-K_{Y}\right)}\right\} \\
& \gamma=\frac{1+v_{Y}}{9\left(1-v_{Y}\right)} \quad, \quad \delta=\frac{4-5 v_{Y}}{15\left(1-v_{Y}\right)}
\end{aligned}
$$

were $\mu_{Y}, K_{Y}$, and $\mu_{N}, K_{N}$ are the shear and bulk moduli of YSZ and Ni, respectively. They are related to the Young's modulus and Poisson's ration through

$$
\mu=\frac{E}{2(1+v)} \quad, \quad K=\frac{E}{3(1-2 v)}
$$

Using the data available for YSZ and Ni, see Table 2-2, we computed the effective properties of the porous $\mathrm{Ni} / \mathrm{YSZ}$ for various porosity levels. The results are shown in Figure 2-7 and Figure 2-8. Our modeling predictions agree very well with the experimental data obtained by E. Lara-Curzio from ORNL, see Figure 2-9.

Table 2-2: Properties of Ni and YSZ

\begin{tabular}{|l|l|l|l|}
\hline & $E(\mathrm{MPa})$ & $v$ & $\alpha\left(10^{-6} /{ }^{0} \mathrm{C}\right)$ \\
\hline $\mathrm{Ni}$ & 200 & 0.3 & 16.0 \\
\hline $\mathrm{YSZ}$ & 200 & 0.3 & 10.56 \\
\hline
\end{tabular}




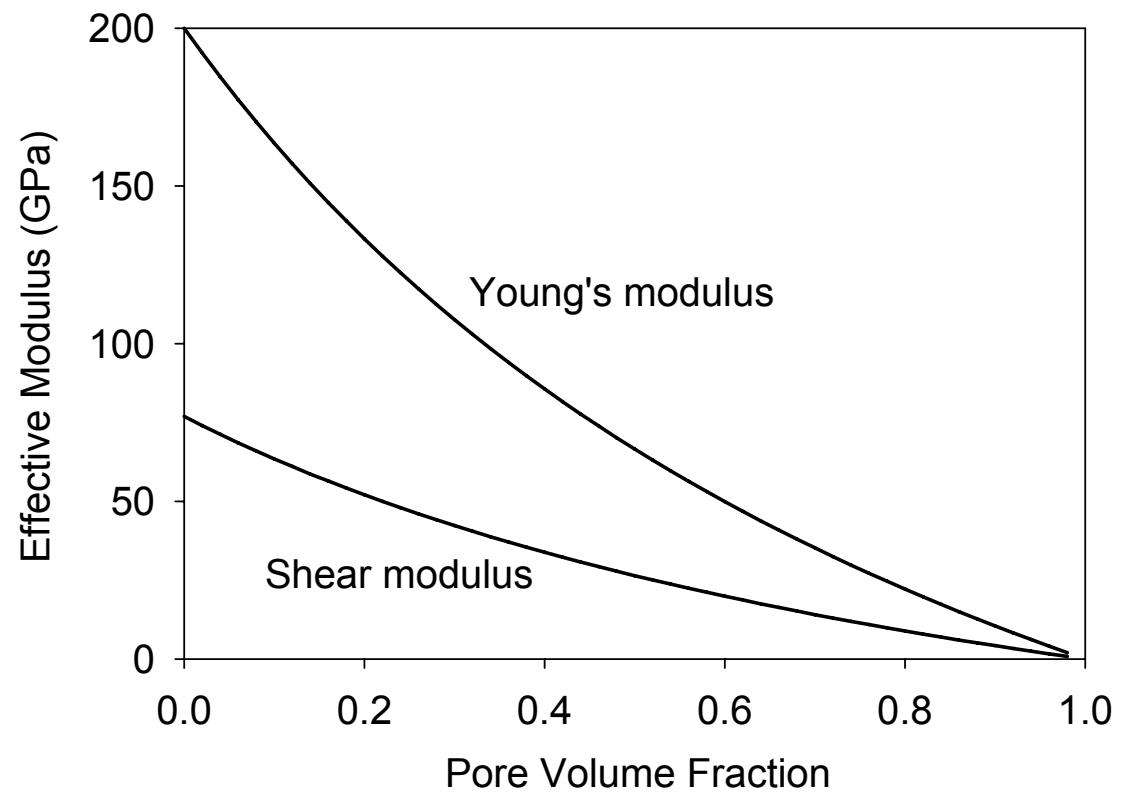

Figure 2-7: Ni/YSZ cermet Young's modulus vs. porosity

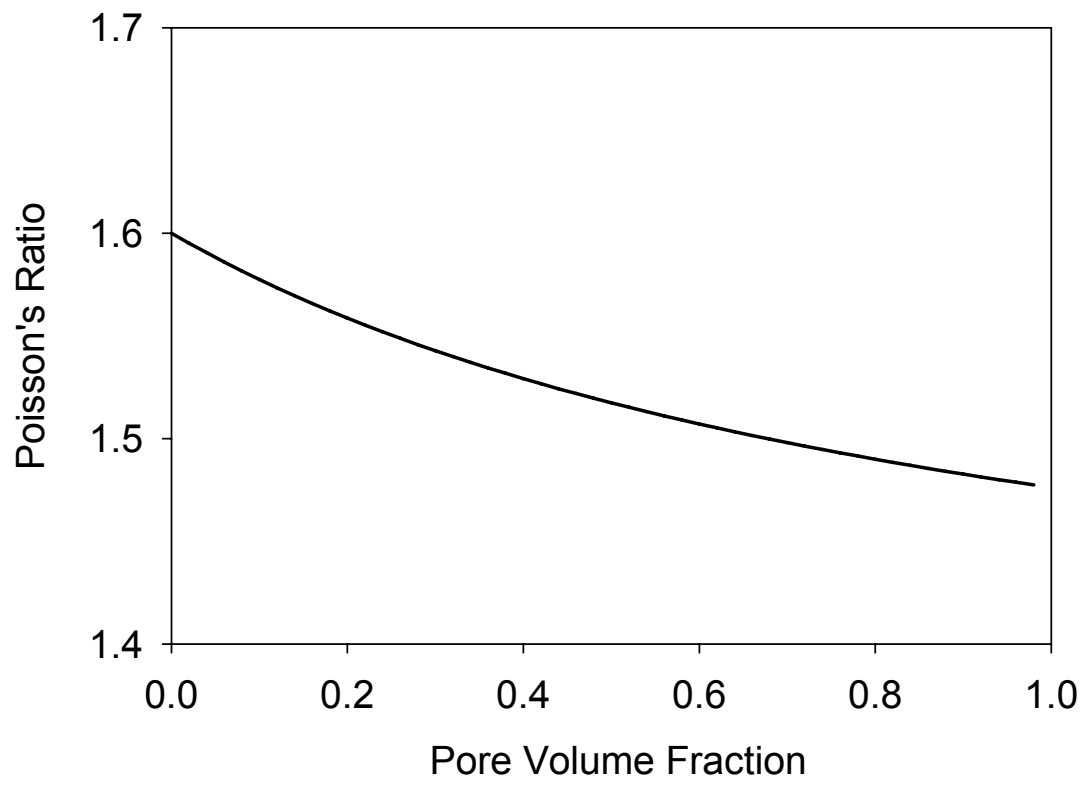

Figure 2-8: Ni/YSZ cermet Poisson's ratio vs. porosity 

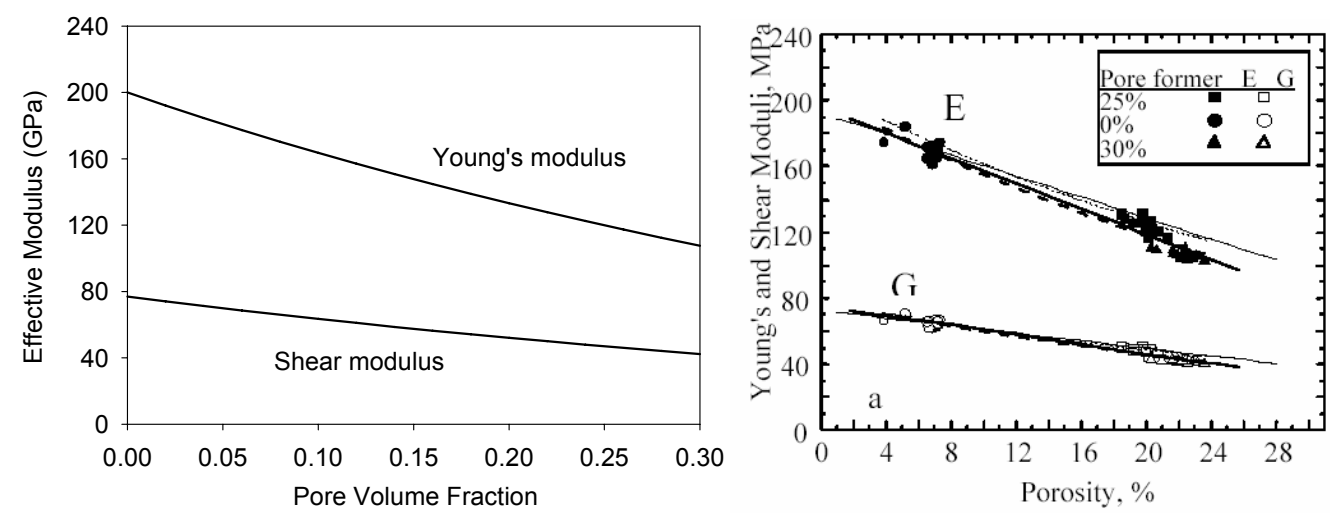

Figure 2-9: Comparison between modeling and experimental results

\subsection{Warpage and Stress Analysis}

We now consider a single square cell of length $2 a$. The seal around the cell is idealized as elastic clamp with modulus $E_{s}$, as shown in Figure 2-10. Let the thickness of the cathode, electrolyte and anode be, respectively, $d_{1}, d_{2}$ and $d_{3}$. Their CTE, modulus and Poisson's ratios are, respectively, $\alpha_{k}, E_{k}$ and $v_{k}(k=1,2,3)$.

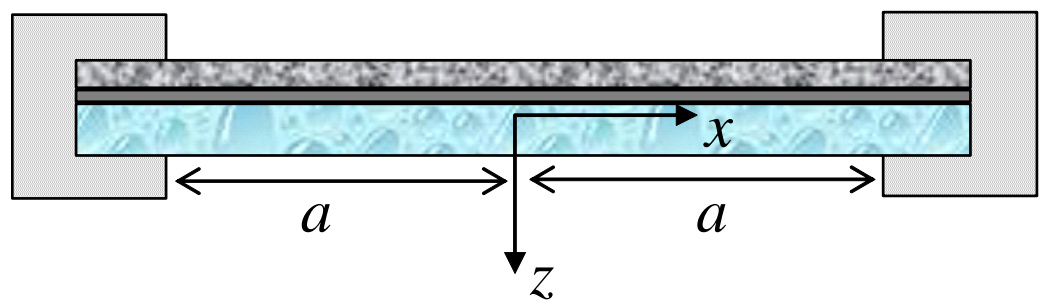

Figure 2-10: A single cell with clamps (seals)

It follows from the derivation in Appendix A that the deflection (warpage) is described by the following equation

$$
w_{0}(x)=\frac{\left(A_{11}+E_{s} h\right) M_{0}-B_{11} N_{0}}{2\left[B_{11}^{2}-\left(D_{11}+a E_{s} h^{2}\right)\left(A_{11}+E_{s} h\right)\right]}\left(a^{2}-x^{2}\right)
$$

where $E_{s}$ is the Young's modulus of the seal, and

$$
A_{11}=\sum_{k=1}^{3} \int_{h_{k}}^{h_{k+1}} \frac{E_{k}}{1-v_{k}^{2}} d z \quad, \quad B_{11}=\sum_{k=1}^{3} \int_{h_{k}}^{h_{k+1}} \frac{E_{k}}{1-v_{k}^{2}} z d z \quad, \quad D_{11}=\sum_{k=1}^{3} \int_{h_{k}}^{h_{k+1}} \frac{E_{k}}{1-v_{k}^{2}} z^{2} d z
$$




$$
\begin{aligned}
& M_{0}=\sum_{k=1}^{3} \int_{h_{k}}^{h_{k+1}} \Delta T(z) \frac{\alpha_{k} E_{k}}{1-v_{k}} z d z, \quad N_{0}=\sum_{k=1}^{3} \int_{h_{k}}^{h_{k+1}} \Delta T(z) \frac{\alpha_{k} E_{k}}{1-v_{k}} d z \\
& h_{1}=-h_{4}=-\frac{d_{1}+d_{2}+d_{3}}{2}, \quad h_{2}=\frac{d_{1}-d_{2}-d_{3}}{2}, \quad h_{3}=\frac{d_{1}+d_{2}-d_{3}}{2}
\end{aligned}
$$

Thus, the corresponding normal stress across the cell thickness follows directly from (C.69),

$$
\sigma_{x}(z)=\frac{E(z)}{1-v^{2}(z)}\left\{\varepsilon_{x}^{0}+z \kappa_{x}-\alpha(z) \Delta T(z)[1+v(z)]\right\}
$$

where the variables that may depend on the z-coordinate are explicitly indicated as functions of $z$, and

$$
\begin{aligned}
& \varepsilon_{x}^{0}=\frac{B_{11} M_{0}-\left(D_{11}+a E_{s} h^{2}\right) N_{0}}{B_{11}^{2}-\left(D_{11}+a E_{s} h^{2}\right)\left(A_{11}+E_{s} h\right)} \\
& \kappa_{x}=\frac{\left(A_{11}+E_{s} h\right) M_{0}-B_{11} N_{0}}{B_{11}^{2}-\left(D_{11}+a E_{s} h^{2}\right)\left(A_{11}+E_{s} h\right)} x^{2}
\end{aligned}
$$

In what follows, numerical results are presented for several specific cases to investigate the effects of different parameters. The parameters used in the baseline mode are listed in Table 2-3.

Table 2-3: Parameters for the baseline mode

\begin{tabular}{|c|c|c|c|c|c|}
\hline & $d(\mathrm{~mm})$ & $E(\mathrm{GPa})$ & $v$ & $\alpha(\mathrm{ppm})$ & $a(\mathrm{~mm})$ \\
\hline $\begin{array}{c}\text { Cathode } \\
(\mathrm{LSM}+\mathrm{YSZ})\end{array}$ & 0.3 & 96 & 0.3 & 10.56 & 50 \\
\hline $\begin{array}{c}\text { Electrolyte } \\
(\mathrm{YSZ})\end{array}$ & 0.01 & 200 & 0.3 & 10.56 & 50 \\
\hline $\begin{array}{c}\text { Anode } \\
(\mathrm{Ni}+\mathrm{YSZ})\end{array}$ & 0.5 & 96 & 0.3 & 12.22 & 50 \\
\hline
\end{tabular}

\subsubsection{Baseline Results}

The baseline model is a free standing single cell. The edges of the cell are not constraint at all. This is equivalent to setting $E_{s}=0$. It is assumed that all the materials are uniform with their dimensions and thermomechanical properties given in Table 2-3. The temperature change $\Delta T=-500^{\circ} \mathrm{C}$ is assumed uniformly through the thickness.

Shown in Figure 2-11 is the deflection curve of the single cell. The maximum deflection is $1.8 \mathrm{~mm}$, occurred in the middle of the cell. Shown in Figure 2-12 is the inplane stress distribution in each layer. It is seen that both the cathode and the electrolyte are subjected to compressive stress, while the anode is under tensile stress. The maximum magnitude of these stresses are $105 \mathrm{MPa}$ in the cathode, $132 \mathrm{MPa}$ in the electrolyte, and $88 \mathrm{MPa}$ in the anode. 


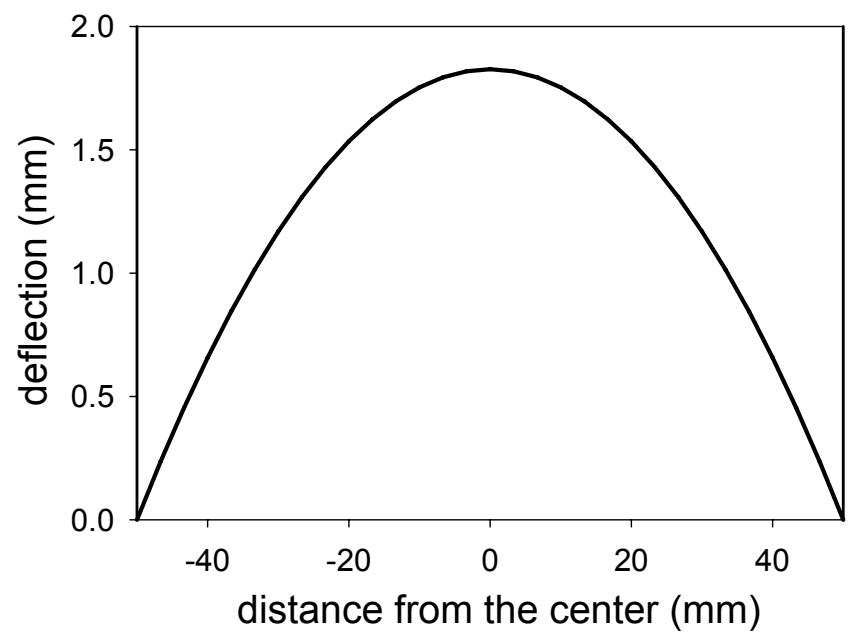

Figure 2-11: Deflection of the cell (baseline model)

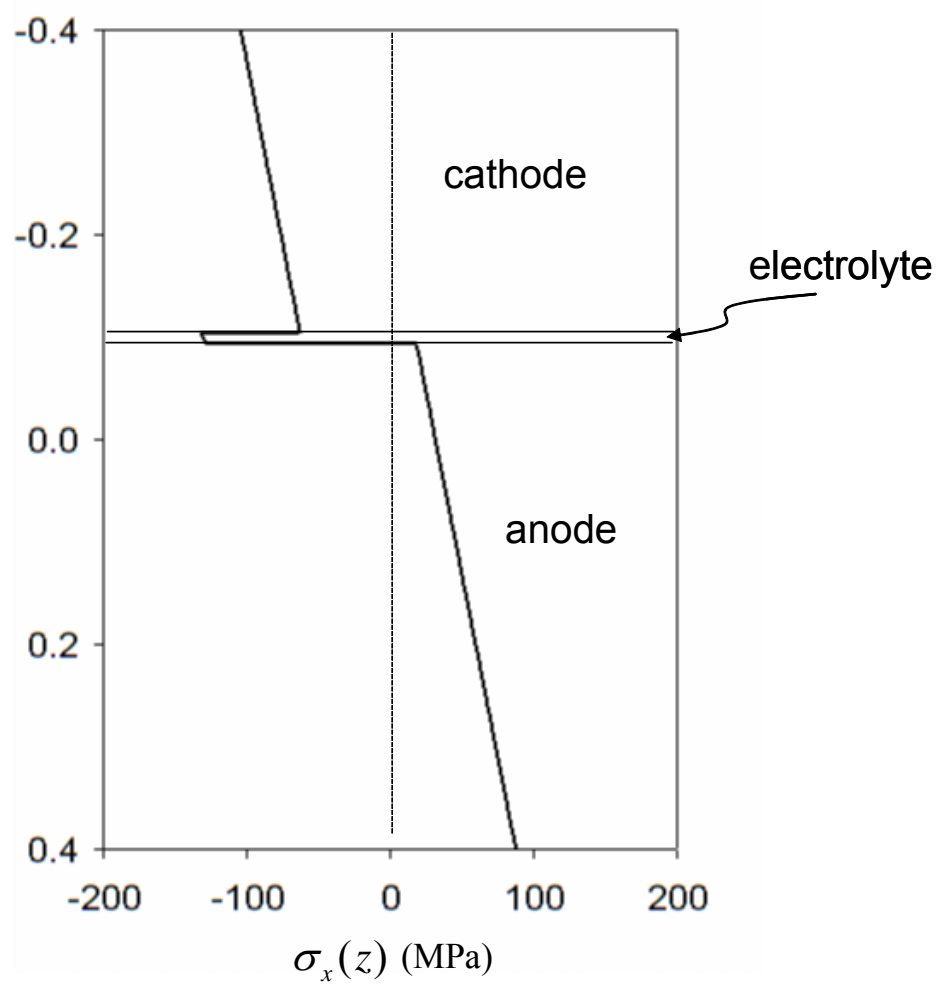

Figure 2-12: In-plane normal stress distribution in each layer (baseline model)

\subsubsection{Effect of Seal's Stiffness}

The maximum deflection of the cell (warpage) was computed for various values of the seal's stiffness, $E_{s}$. Shown in Figure 2-13 is the change of the maximum defection for $E_{s}$ between 0 and $300 \mathrm{GPa}$. Note the vertical axis is plotted in logarithm scale. It can be seen that the deflection reduces dramatically from $\mathrm{mm}$ to several micrometers 
when $E_{s}$ is increase from 0 to $50 \mathrm{GPa}$, indicating that the warpage can be effectively controlled by using stiffer seals, or clamps.

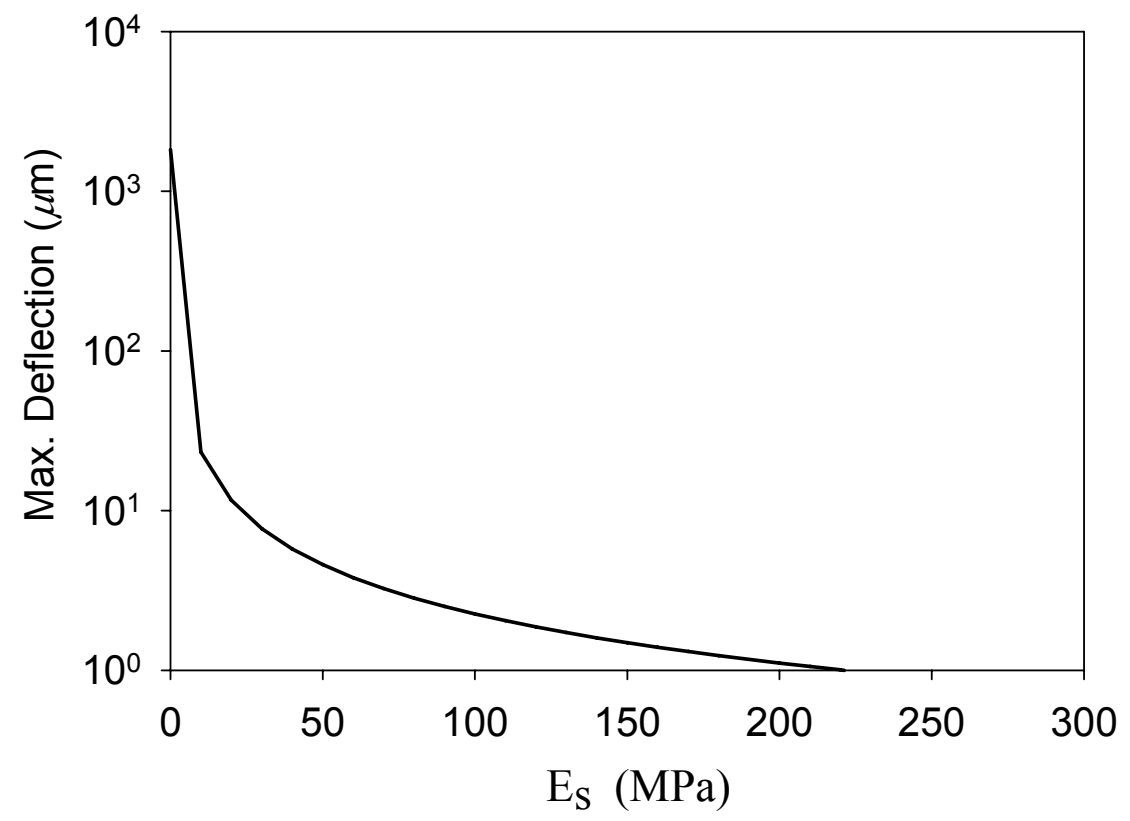

Figure 2-13: Maximum deflection vs. seal's stiffness

However, as the seals stiffness increases, so do the stresses in the cell. Shown in Figure 2-14 are the values of maximum in-plane normal stress, $\sigma_{x}$, in the cell, as functions of the seal's stiffness. It is seen that stresses increase significantly as the seal becomes stiffer.

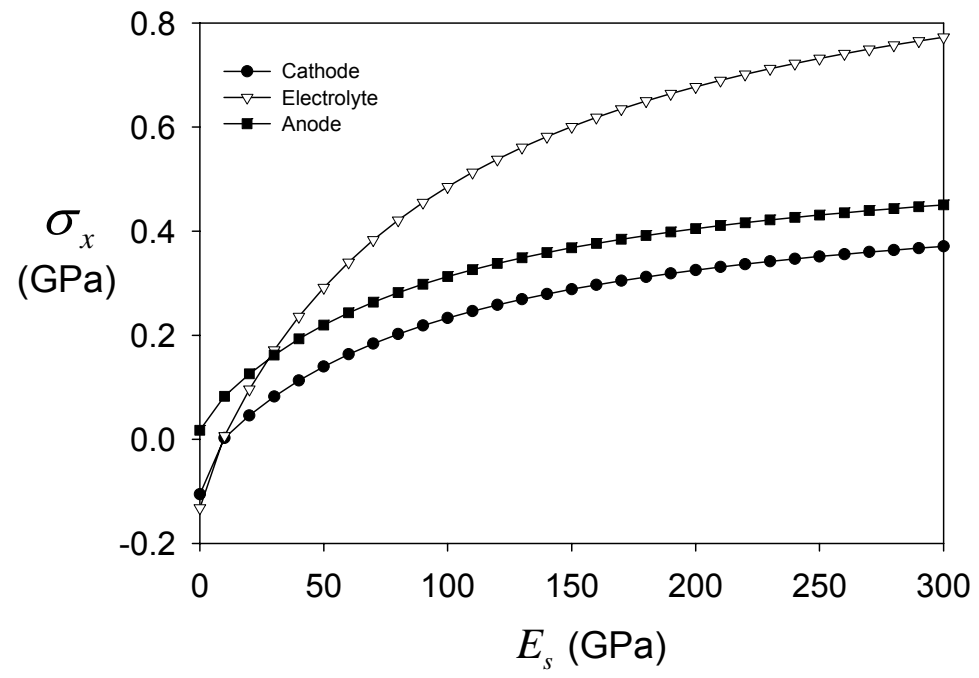

Figure 2-14: Cell stress vs. seal stiffness 


\subsubsection{Effect of Nonuniform Anode Porosity}

To investigate how the graded anode will affect the stresses and warpage, a numerical experiment was conducted. It is assumed that the porosity in the anode changes linearly from $10 \%$ at the anode/electrolyte interface to $50 \%$ at the anode/interconnect interface. The corresponding modulus of the graded anode was computed from (2.11).

Shown in Figure 2-15 is the effect of graded anode on the maximum deflection. Clearly, the graded anode increases the warpage. The maximum stress in each layer is shown in Figure 2-16 through Figure 2-18. It is seen that the maximum stress decreased slightly in both the cathode and electrolyte layers, but increase dramatically in the anode if the anode is graded.

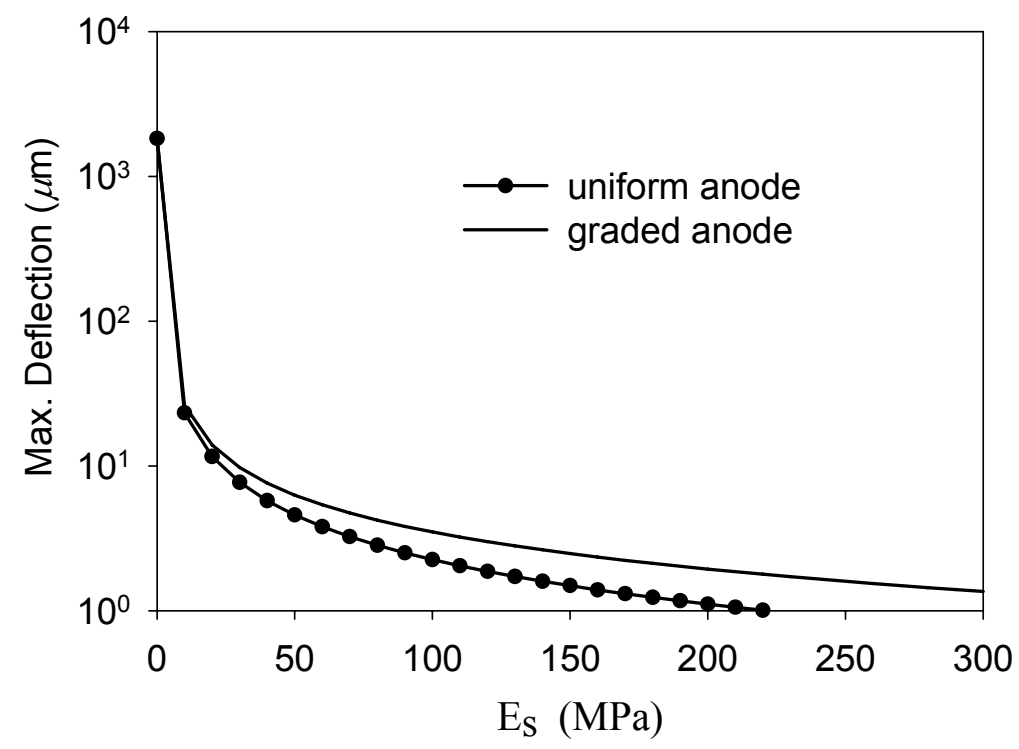

Figure 2-15: Effects of graded anode on the warpage 


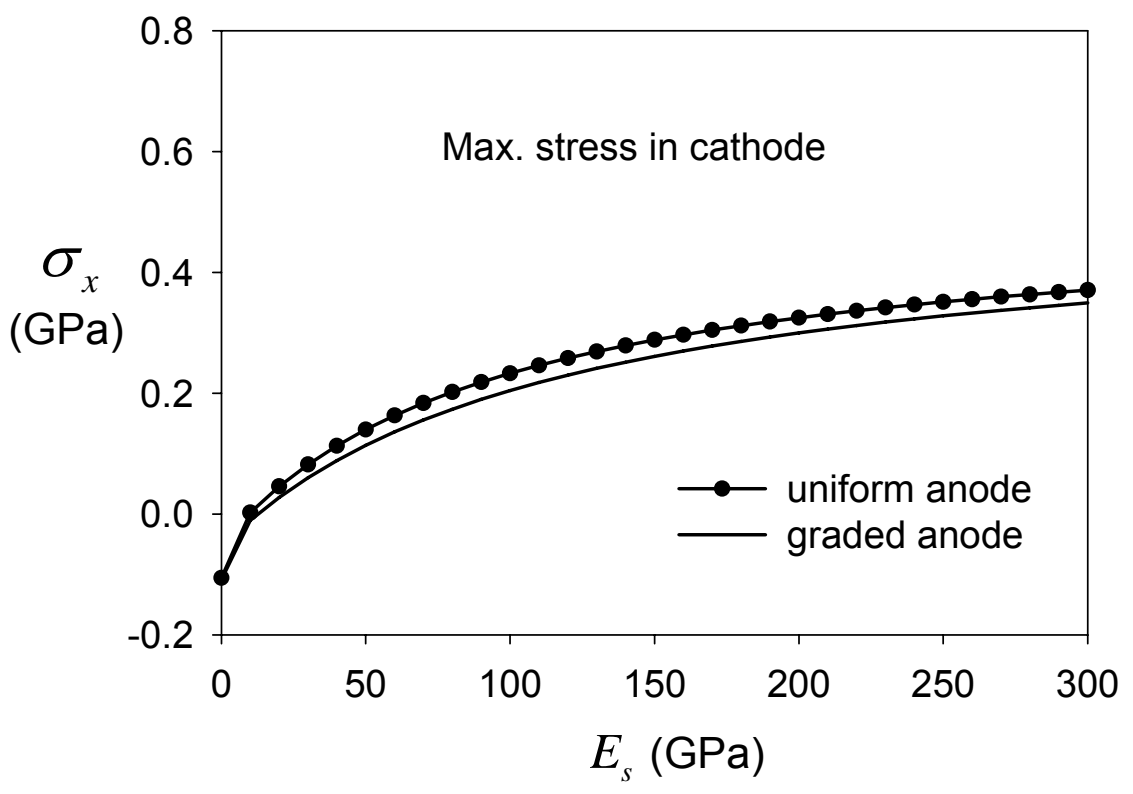

Figure 2-16: Effect of graded anode on the maximum stress in the cathode

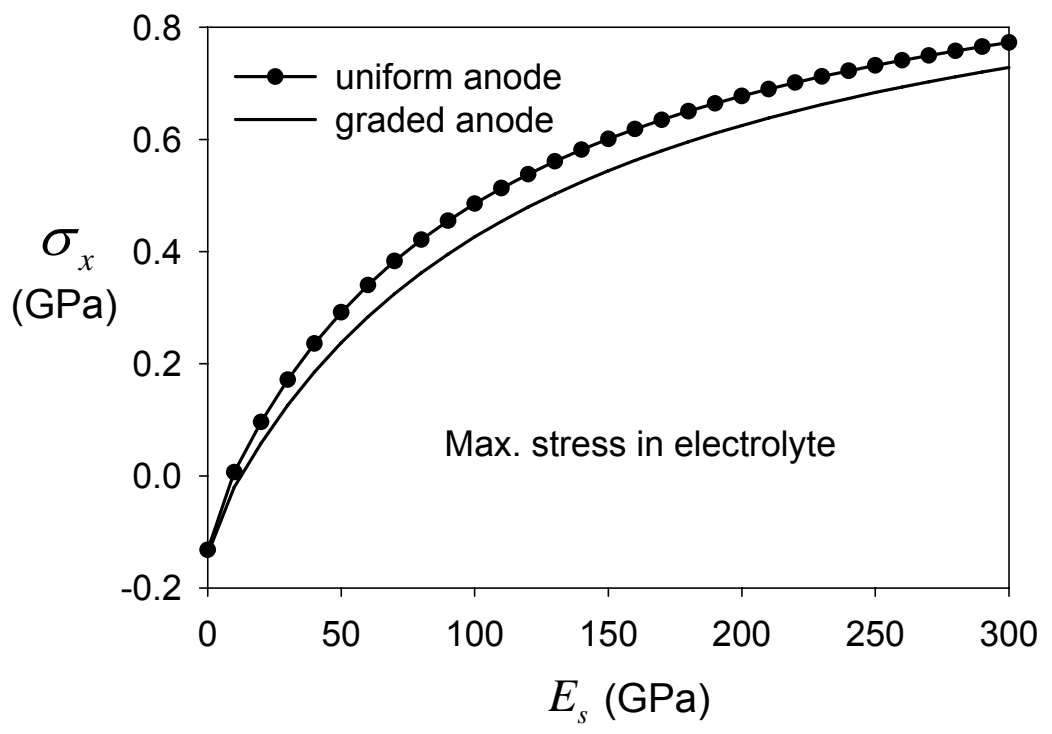

Figure 2-17: Effect of graded anode on the maximum stress in the electrolyte 


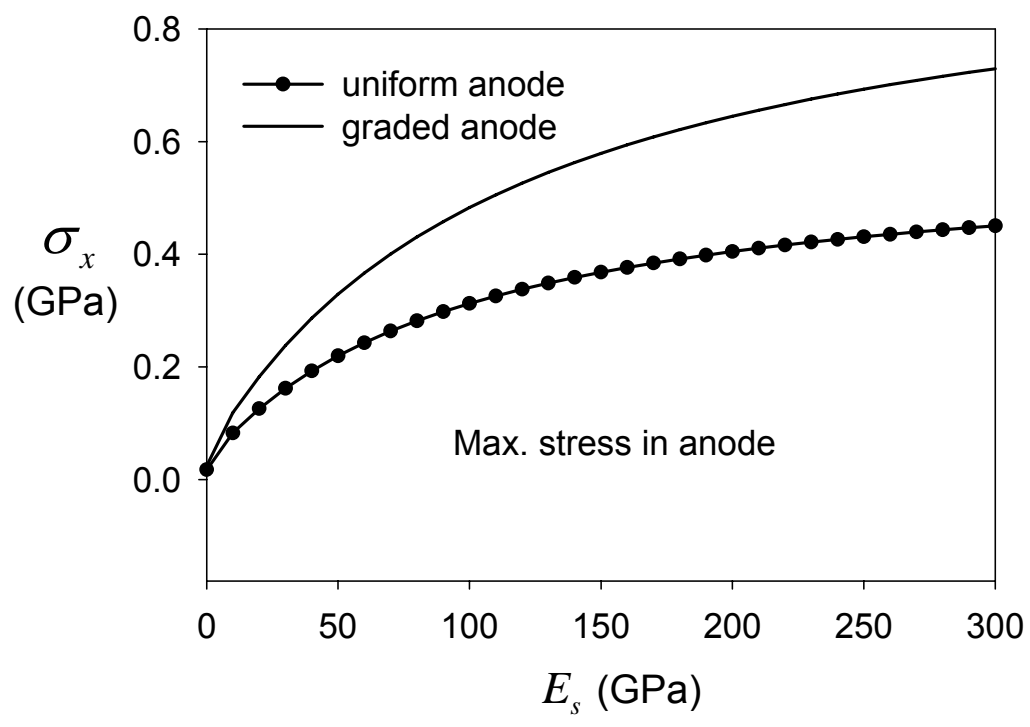

Figure 2-18: Effect of graded anode on the maximum stress in the anode

\subsection{Spalling Phenomenon during Thermal Transients and Shock}

During start-up and shot-down, cells are subjected to thermal shocks. The rapid change of temperature can produce significant thermal stresses in the electrodes. If not managed properly, such thermal stresses may result in microcracks in the electrodes. The objective of this work is to develop a model to quantitatively understand how microcracks in the electrodes are created during transient thermal loading. For simplicity, residual stresses are neglected.

It was derived in Appendix B that microcrack density, $\mathrm{Nb}^{3}$, in a material is related to the heating rate (Joule heating), $q$, through,

$$
q=\frac{3 \pi^{2} k r_{0}}{2 \alpha}\left[1+\frac{16\left(1-v^{2}\right) N b^{3}}{9(1-2 v)}\right] \sqrt{\frac{G_{c} \pi(1-v)}{E_{0} b(1+v)}}
$$

where

$E_{0}=$ Elastic Young's modulus of the un-cracked material

$v=$ Poisson's ratio of the un-cracked material

$G_{c}=$ Fracture toughness of the material

$b=$ crack size

$N=$ number of cracks per unit volume

$k=$ Thermal diffusivity

$\alpha=$ Coefficient of linear thermal expansion 
In the above, $r_{0}$ is a length parameter that characterizes the spatial non-uniformity of the heat source, see Eq. (B.4). Smaller $r_{0}$ means less uniform.

The following can be observed from (2.25).

(a) For a given material and spatial heat source distribution, the microcrack density is proportional to the heating rate. Furthermore, there seems to be a threshold value for heating rate

$$
q_{t h}=\frac{3 \pi^{2} k r_{0}}{2 \alpha} \sqrt{\frac{G_{c} \pi(1-v)}{E_{0} b(1+v)}}
$$

below which the crack density is negligible. This threshold heating rate is proportional to the thermal conductivity, i.e., the faster thermal energy conducts through the heating area, the smaller the tendency of microcrack generation.

Table 2-4: Thermomechanical properties of Ni/YSZ

\begin{tabular}{|l|l|l|l|l|}
\hline$E(\mathrm{~Pa})$ & $v$ & $G_{c}\left(\mathrm{~J} / \mathrm{m}^{2}\right)$ & $k(\mathrm{~J} /(\sec \mathrm{m} \mathrm{K}))$ & $\alpha(1 / \mathrm{K})$ \\
\hline $96 \times 10^{9}$ & 0.3 & 9 & 5.84 & $12.22 \times 10^{-6}$ \\
\hline
\end{tabular}

(b) The allocable heating rate is proportional to the square root of the materials fracture toughness. Shown in Figure 2-19 is the relationship between fracture toughness and maximum allowable heating rate for various values of microcrack densities.

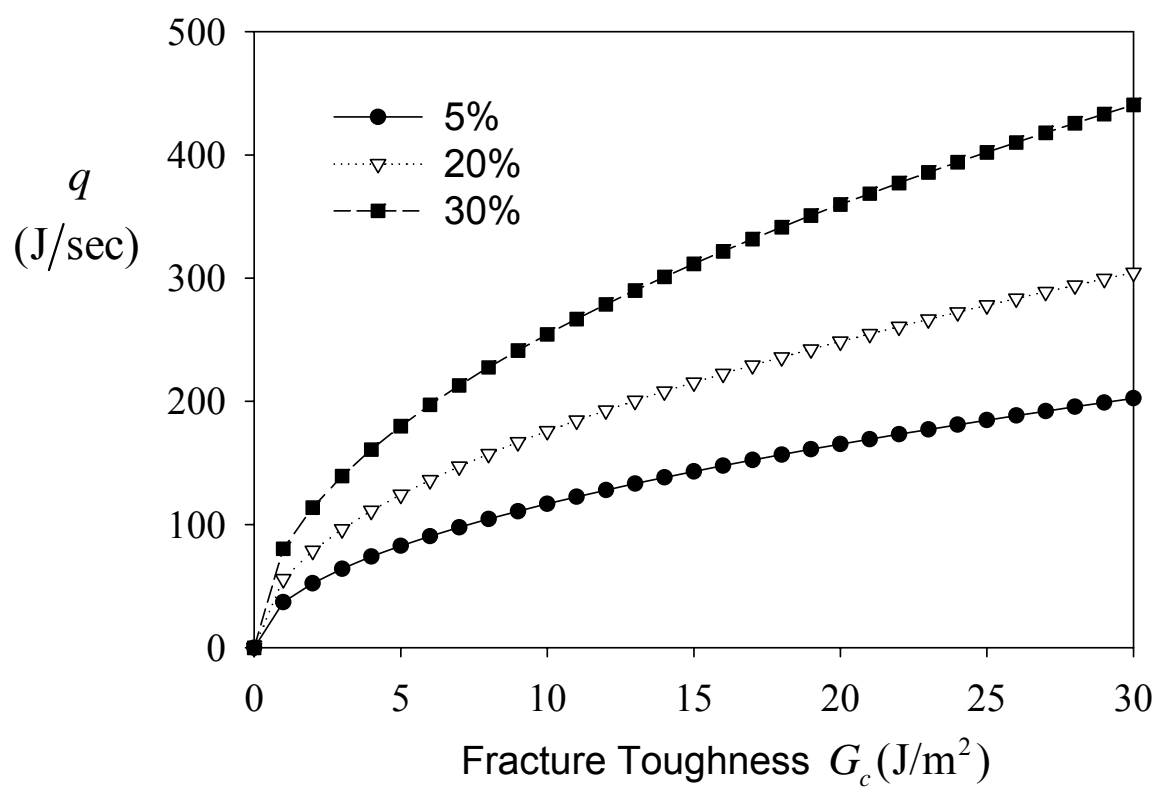

Figure 2-19: Maximum allowable heating rate vs. fracture toughness for various amount of porosity 


\subsection{D Finite Element Analysis of Crack Path Selection and Propagation}

Near the crack tip, stresses are extremely high. This may cause material failure. Crack initiation and growth are the most commonly observed thermomechanical failure modes in SOFCs. In this task, we focus on developing methodologies to (1) calculate crack tip singular stress fields and (2) identify cracking paths.

Based on linear elastic fracture mechanics, the stresses near a crack tip can be written as

$$
\begin{aligned}
\sigma_{x} & =\frac{K_{I}}{\sqrt{2 \pi r}}\left[1-\sin \frac{\theta}{2} \sin \frac{3 \theta}{2}\right] \cos \frac{\theta}{2}-\frac{K_{I I}}{\sqrt{2 \pi r}}\left[2+\cos \frac{\theta}{2} \cos \frac{3 \theta}{2}\right] \sin \frac{\theta}{2}, \\
\sigma_{y} & =\frac{K_{I}}{\sqrt{2 \pi r}}\left[1+\sin \frac{\theta}{2} \sin \frac{3 \theta}{2}\right] \cos \frac{\theta}{2}+\frac{K_{I I}}{\sqrt{2 \pi r}} \sin \frac{\theta}{2} \cos \frac{\theta}{2} \cos \frac{3 \theta}{2} \\
\tau_{x y} & =\frac{K_{I}}{\sqrt{2 \pi r}} \sin \frac{\theta}{2} \cos \frac{\theta}{2} \cos \frac{3 \theta}{2}+\frac{K_{I I}}{\sqrt{2 \pi r}}\left[1-\sin \frac{\theta}{2} \sin \frac{3 \theta}{2}\right] \cos \frac{\theta}{2}
\end{aligned}
$$

where the stress intensity factors, $K_{I}$ and $K_{I I}$, need to be calculated numerically for each set of given boundary conditions and applied loads.

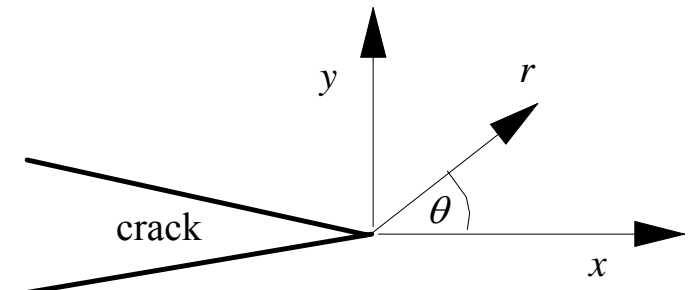

Figure 2-20: Crack-tip coordinate system.

For a given set of boundary conditions and loads, an existing crack will grow if the following condition is met

where

$$
G \geq G_{i c}
$$

$$
G=\frac{1-v^{2}}{E}\left(K_{I}^{2}+K_{I I}^{2}\right)
$$

is called the energy release rate and $G_{i c}$ is the fracture toughness, an intrinsic material property that must be measured experimentally. Literature values of $G_{i c}$ for Ni/YSZ cermat is around $10 \mathrm{~J} / \mathrm{m}^{2}$, for YSZ is about $30 \mathrm{~J} / \mathrm{m}^{2}$.

To calculate the energy release rate, the finite element method was used. In particular, the virtual crack extension method was implemented in the commercial code ANSYS for this purpose. Briefly, to calculate the energy release rate for a given crack of length $a$, a finite element model was generated first. Then calculations were carried out to obtain the total strain energy, $U(a)$, in the model. Next, the crack was virtually 
extended by $\Delta a$ and new calculations were performed to obtain the total strain energy, $U(a+\Delta a)$ in the new model. The crack tip energy release rate is thus given by

$$
G=\frac{U(a+\Delta a)-U(a)}{\Delta a}
$$

In the finite element calculations, attention must be paid to crack tip meshing. Because of the large stress gradient, very fine mesh must be used near the crack tip. Furthermore, due to stress singularities, singular elements must be used around the crack tip. In our analysis, skewed quarter-point quadrilateral elements (degenerated to triangular elements) were used around the crack tip. In addition, the virtual crack extension was realized by shifting (in the direction of crack growth) all the crack-tip nodes within a radius of one-half of the crack length by $1 \%$ of the crack length.

Based on the fracture criterion stated by (2.30), the propensity of crack growth under a given condition can be determined once the energy release rate near the crack tip is calculated. In the following, we will study two crack configurations to determine the crack growth behavior. The properties of the materials used in the calculations are listed in Table 2-1.

Table 2-5: Properties used in the finite element analysis

\begin{tabular}{|c|c|c|c|c|}
\hline Materials & $\begin{array}{c}\text { Young's } \\
\text { modulus (GPa) }\end{array}$ & Poisson's ratio & $\begin{array}{c}\text { CTE } \\
\left.6 /{ }^{\circ} \mathrm{C}\right)\end{array}$ & $\begin{array}{c}\text { Layer thickness } \\
(\mathrm{mm})\end{array}$ \\
\hline $\begin{array}{c}\text { Cathode } \\
(\mathrm{LSM}+\text { YSZ) }\end{array}$ & 96 & 0.3 & 10.56 & 0.3 \\
\hline $\begin{array}{c}\text { Electrolyte } \\
(\mathrm{YSZ})\end{array}$ & 200 & 0.3 & 10.56 & 0.01 \\
\hline $\begin{array}{c}\text { Anode } \\
(\mathrm{Ni}+\mathrm{YSZ})\end{array}$ & 96 & 0.3 & 12.22 & 1.00 \\
\hline
\end{tabular}

\subsubsection{Crack Growth within Anode}

A two dimensional single cell model was created using ANSYS. Due to symmetry, only one-half of the cell was meshed, as shown in Figure 2-21. The line of symmetry is simulated in the model by the "roller condition". The model was meshed with 8 node quadrilateral elements, see Figure 2-22. The stress singularity at each crack tip was modeled with skewed quarter-point quadrilateral elements degenerated to triangular elements. Two rows of elements were created each with radius of $1.25 \mu \mathrm{m}$. The initial crack was placed perpendicular to the electrode layers at a distance of $0.25 \mathrm{~mm}$ from the cell center and $0.89 \mathrm{~mm}$ from the bottom of the anode. The total length of the model was $1.5 \mathrm{~mm}$. The crack growth was only allowed to extend toward the electrolyte since the compressive stresses at the bottom of the anode were shown to halt crack growth.

It should be noted that although the stress field may vary significantly through the thickness of the cell, it is almost uniform along the cell. The existence of a small crack is felt only by the immediate area surrounding the crack. Therefore, when study crack behavior, it suffices to model a small region near the crack. This is why in this 
calculation, as well as in the edge delamination analysis, we only took a small portion of the total cell.

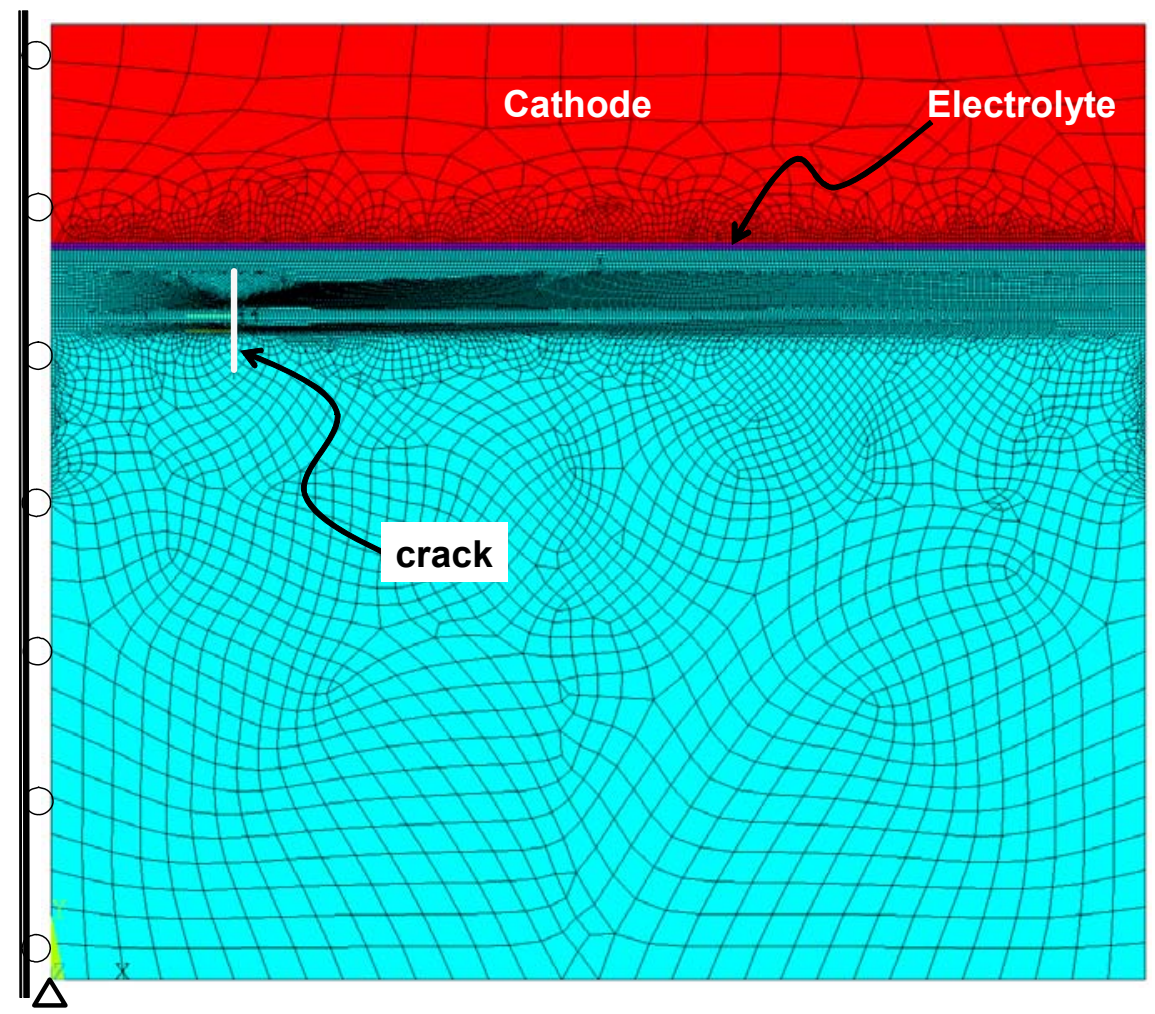

Figure 2-21: A crack embedded in the anode grow towards the electrolyte

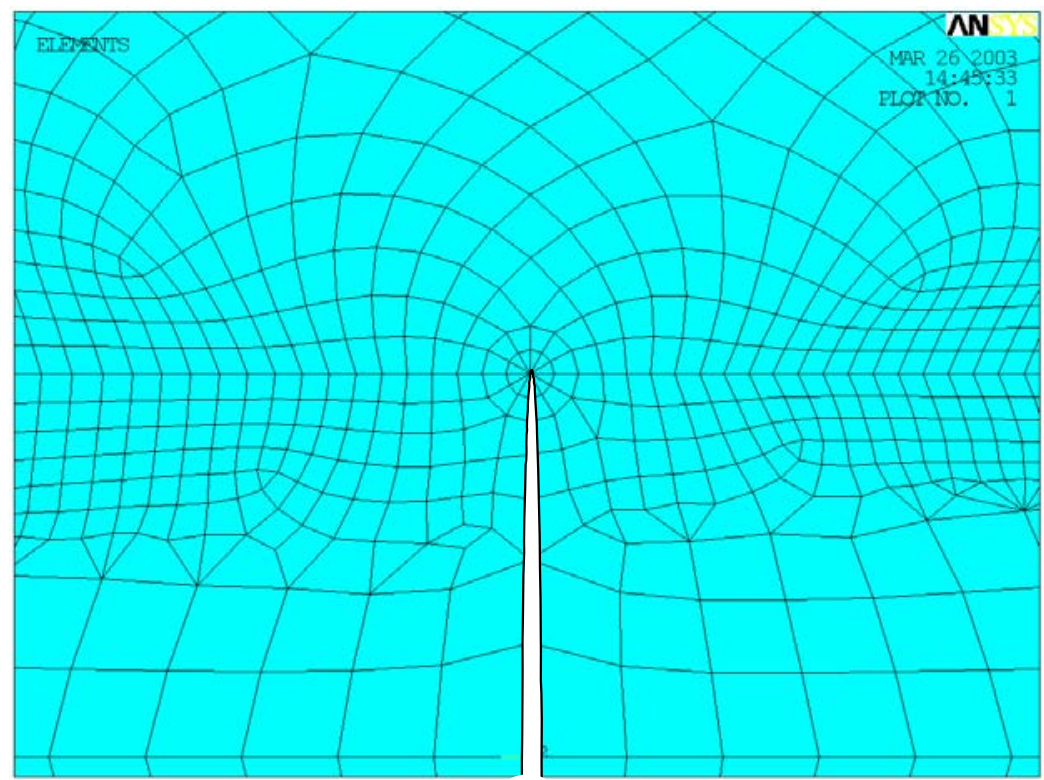

Figure 2-22: An enlarged view of the crack tip mesh shown in Figure 2-21. 
A convergence study was conducted to verify that the above mesh configuration provided consistent stress intensity values and energy release rate. The results for a temperature change of $1000^{\circ} \mathrm{C}$ are given in Table 2-6.

Table 2-6: Crack tip stress intensity factors and energy release rate.

\begin{tabular}{|c|c|c|c|c|}
\hline $\begin{array}{c}\text { Crack Size } \\
(\mu \mathrm{m})\end{array}$ & $\begin{array}{c}\text { Distance from } \\
\text { Electrolyte }(\mu \mathrm{m})\end{array}$ & $\begin{array}{c}K_{I} \\
(\mathrm{MPa} \sqrt{\mathrm{m}})\end{array}$ & $\begin{array}{c}K_{I I} \\
(\mathrm{MPa} \sqrt{\mathrm{m}})\end{array}$ & $\begin{array}{c}G \\
\left(\mathrm{~J} / \mathrm{m}^{2}\right)\end{array}$ \\
\hline 20 & 90 & 0.582 & 0.009 & 3.21 \\
\hline 30 & 80 & 0.725 & 0.011 & 4.98 \\
\hline 40 & 70 & 0.849 & 0.013 & 6.84 \\
\hline 50 & 60 & 0.960 & 0.014 & 8.73 \\
\hline 90 & 20 & 1.338 & 0.019 & 16.97 \\
\hline 105 & 5 & 1.396 & 0.017 & 18.49 \\
\hline
\end{tabular}

Based on the data shown in Table 2-6, two conclusions can be drawn. First, the maximum tolerable initial flaw size is around $50 \mu \mathrm{m}$, if the toughness of the anode is 10 $\mathrm{J} / \mathrm{m}^{2}$, a typical value for $\mathrm{Ni} / \mathrm{YSZ}$. Flaw size greater than $50 \mu \mathrm{m}$ may result in fracture. Secondly, once fracture occurs, the crack will grow toward the electrolyte layer in an unstable fashion, for the energy release rate increases as the crack getting closer to the electrolyte layer.

The existence of crack also alters the local stress fields. Shown in Figure 2-23 is the comparison of the stresses with and without the crack.

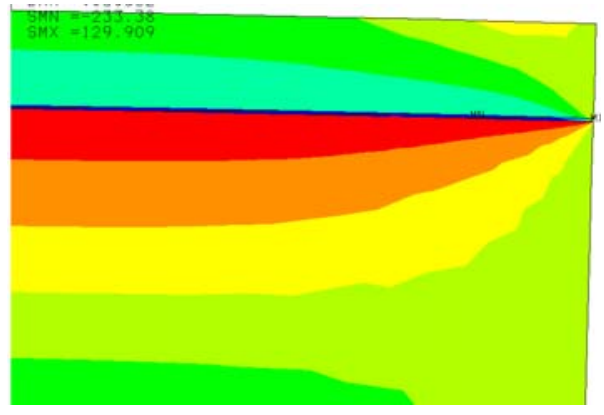

(a)

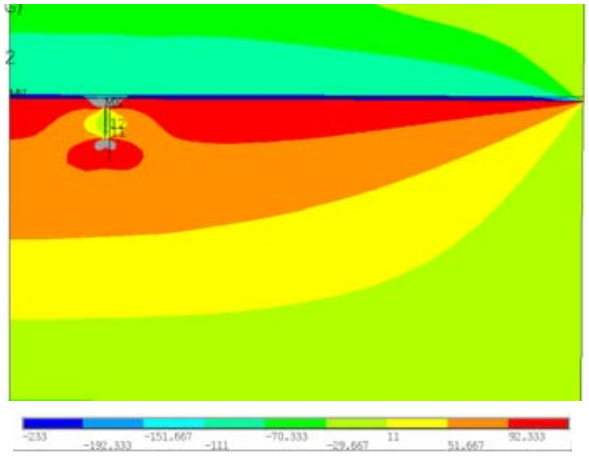

(b)

Figure 2-23: Comparison of stress $\left(\sigma_{x}\right)$ between cells with (b) and without (a) existing crack.

\subsubsection{Behavior of Edge Delamination}

Unlike cracks in the anode that most likely will lead to the fracture of electrolyte, an edge delamination has the propensity of either continuing along the interface, or be deflected into the electrolyte or the anode, as illustrated in Figure 2-24. 

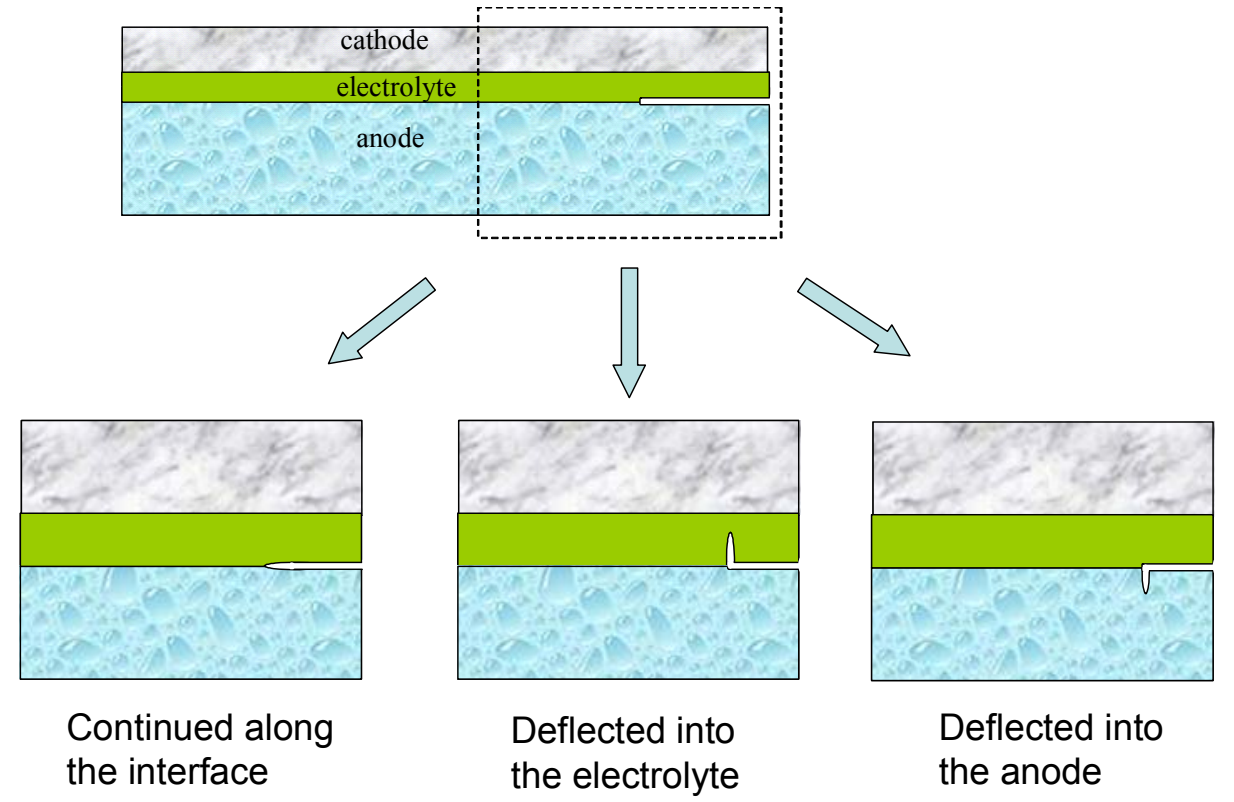

Figure 2-24: Possible cracking paths for an edge delamination.

To determine the crack path, let the fracture toughness of the anode, electrolyte and their interface be denoted by $G_{c}^{a}, G_{c}^{e}$, and $G_{c}^{i}$, respectively. Although the exact values of these material properties need to be experimentally determined, literature data seem to indicate that for typical Ni/YSZ anode and YSZ electrolyte,

$$
G_{c}^{i} \leq G_{c}^{a} \leq G_{c}^{e} .
$$

Let the energy release rates for cracks deflected into the anode, electrolyte and continued along the interface be, respectively, $G^{a}, G^{e}$ and $G^{i}$. Once these values are computed using the finite element method, possible cracking path can be determined based on the following criteria:

(1) between continuing along the interface and going into electrolyte

$$
\frac{G^{e}}{G^{i}}>\frac{G_{c}^{e}}{G_{c}^{i}} \Rightarrow \text { going into electrolyte }
$$

(2) between continuing along the interface and going into anode

$$
\frac{G^{a}}{G^{i}}>\frac{G_{c}^{a}}{G_{c}^{i}} \Rightarrow \text { going into anode }
$$

(3) between going into anode and going into electrolyte

$$
\frac{G^{e}}{G^{a}}>\frac{G_{c}^{e}}{G_{c}^{a}} \Rightarrow \text { going into electrolyte }
$$

To calculate the energy release rates $G^{a}, G^{e}$ and $G^{i}$, ANSYS models were created for a single cell with an existing edge delamination of $50 \mu \mathrm{m}$ along the anode/electrolyte 
interface. The mesh is shown in Figure 2-25. Using the virtual crack extension method, we computed the energy release rate for a temperature loading of $1000^{\circ} \mathrm{C}$. The results are listed in Table 1.3. Make use of these data, in conjunction with the crack path selection criteria, one can conclude that the edge delamination will not cause fracture of the electrolyte unless the fracture toughness of the electrolyte is less than $\sim 30 \%$ of that of the interface or that of the anode. This is typically not the case for Ni/YSZ anode and YSZ electrolyte. Therefore, for YSZ planar cells, edge delamination is likely to grow either along the interface or grow into the anode, both are less catastrophic than electrolyte fracture which leads to fuel leakage.

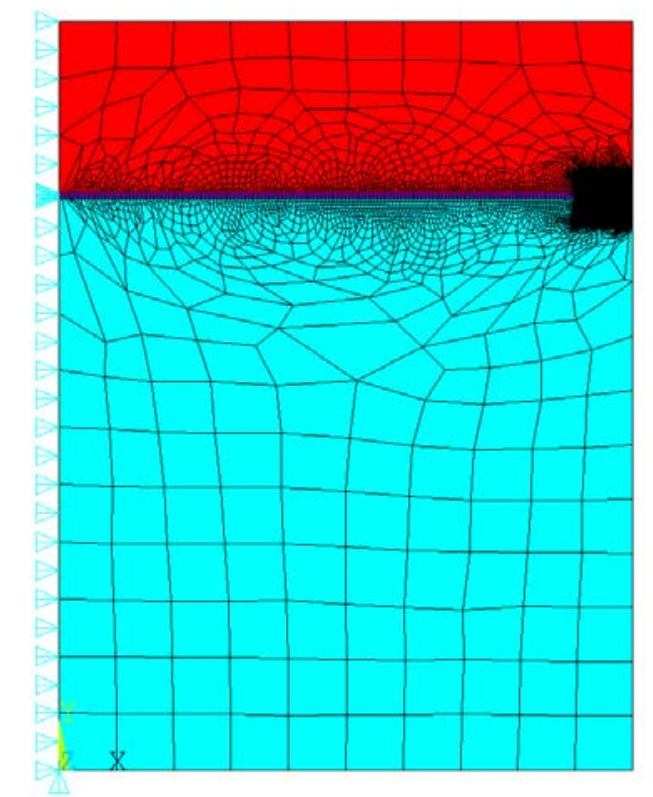

Figure 2-25: Finite element mesh for an edge delamination.

Table 2-7: Energy release rate for various crack paths from an edge delamination

\begin{tabular}{|c|c|}
\hline Path & Energy Release Rate $\left(\mathrm{J} / \mathrm{m}^{2}\right)$ \\
\hline$G^{e}$ & 3.24 \\
\hline$G^{i}$ & 10.12 \\
\hline$G^{a}$ & 11.61 \\
\hline
\end{tabular}

\subsection{Fracture Mechanical Analyzer (FMA) - Theory and Background}

While fuel cells are undergoing renewed development in industry due to their compact configuration, high efficiency and low emissions. The high costs and unproven durability still remain as common industry concerns. This is due to electrochemical and thermomechanical reactions and strict structural requirements, making long term operation of planar solid oxide fuel cells (SOFCs) extremely difficult. To address this, numerical models have successfully incorporated electrochemistry and structural stresses, 
but detailed fracture analyses have yet to be performed [1,2]. The recent shift of SOFCs to anode-supported structures, in which a thin film electrolyte is sintered onto the anode support, has further hampered structural modeling due to the large aspect ratio between the anode/electrolyte layers and cell length. In a fracture analysis there is also difficulty in modeling fracture at areas of interest, i.e. the PEN (anode-electrolyte-cathode layer) region or at the seal interfaces. Yet due to thermal mismatch between these layers and the cell operating conditions, significant thermal stresses are created within the cell structure, which may eventually lead to failure, making fracture analysis a critical part of thermomechanical modeling of SOFCs. Specifically, simulation tools are needed to obtain fracture mechanics parameters such as the stress intensity factors (SIFs), and to understand the influence of thermal gradients on crack behavior.

To meet this need, a computer program called Fracture Mechanical Analyzer (FMA) was developed to calculate the SIFs of 3D cracks, including interfacial cracks in the PEN structure subjected to combined mechanical and thermal loadings [3]. The FMA program, written in MatLab language, is essentially an "add-on" to any commercial finite element software. It computes the energy release rate and the individual SIFs based on the crack-tip displacement fields computed from any commercial finite element software. To illustrate its usage and capabilities, the FMA program is used here in conjunction with the finite element software ANSYS to study various crack geometries occurring in the PEN layer

\subsubsection{Fracture Mechanics}

The study of crack growth in materials is characterized by two different fracture parameters; an energy based parameter and a stress intensity approach [4]. The fracture parameters will vary depending on the loading conditions and the body configuration. Once the fracture parameter is calculated it can be compared to the material's resistance to crack growth to determine if the crack will grow.

An energy based parameter, energy release rate $(G)$, is defined as the energy required for crack growth. This parameter assumes that a crack increases when the energy available for crack extension overcomes the inherent resistance of the material. Equation (2.34) is a mathematical interpretation of this value, where $d \pi$ is the change of potential energy in the system with respect to the differential crack growth $(d a)$ [4].

$$
G=-\frac{d \pi}{d a}
$$

While the energy release rate can predict crack growth it does not allow for the definition of the stress fields near the crack tip. Stresses near the crack tip will be extremely high, or mathematically a stress singularity will occur at the tip of the crack [4]. Characterization of this stress intensity can be used to completely define the stresses occurring near the crack tip. The stress intensity factor is a constant that is proportional to the stresses occurring near the crack tip. The parameter is important because it can be separated into loading modes designating opening $\left(K_{I}\right)$, in-plane shear $\left(K_{I I}\right)$, and out of plane shear $\left(K_{\text {III }}\right)[4]$. 
The energy release rate and the stress intensity factors are related to each other as shown below:

$$
G=\frac{K_{I}^{2}+K_{I I}^{2}}{E^{\prime}}+\frac{K_{I I I}^{2}}{2 \mu}
$$

The Young's modulus $(E)$ and shear toughness $(\mu)$ are defined in (2.36).

$$
\begin{aligned}
& E^{\prime}=E \text { plane stress } \\
& E^{\prime}=\frac{E}{1-v^{2}} \text { for plane strain } \\
& \mu=\frac{E^{\prime}}{2(1+v)}
\end{aligned}
$$

While interfacial cracking can also be characterized by energy release rate and the stress intensity factors, its study is complicated by the oscillatory behavior of the crack tip singularity. This requires introductions of several other fracture parameters. For instance, elastic material parameters are needed to characterize mismatch. The Dunder's parameters $(\alpha, \beta)$ characterize elastic mismatch for isotropic solids, where $k_{i}=3-4 v_{i}$ for plane strain and $k_{i}=\left(3-v_{i}\right) /\left(1+v_{i}\right)$ for plane stress, where $i$ designates the material number.

$$
\begin{array}{r}
\alpha=\frac{\mu_{1}\left(\kappa_{2}+1\right)-\mu_{2}\left(\kappa_{1}+1\right)}{\mu_{1}\left(\kappa_{2}+1\right)+\mu_{2}\left(\kappa_{1}+1\right)} \\
\beta=\frac{\mu_{1}\left(\kappa_{2}-1\right)-\mu_{2}\left(\kappa_{1}-1\right)}{\mu_{1}\left(\kappa_{2}+1\right)+\mu_{2}\left(\kappa_{1}+1\right)}
\end{array}
$$

The bimaterial constant $(\varepsilon)$ is based on the Dunder's constants as is defined in equation (2.37). The bimaterial constant is sometimes referred to as the oscillation index because the size relates to the behavior of the complex stress intensity factor $\left(\mathbf{K}=K_{I}+i K_{I I}\right)$ used by Rice and Sou [5].

$$
\varepsilon=\frac{1}{2 \pi} \ln \left(\frac{1-\beta}{1+\beta}\right)
$$

The stress fields at the crack tip can be written as a function of the intensity factors if the bimaterial constant is used as an oscillation index [5]. If the bimaterial constant is zero, which occurs if material one and two are equivalent, the bimaterial stress field in equation (2.39) will reduce to the homogenous form in (2.27)-(2.29).

$$
\sigma_{i j}=\frac{\operatorname{Re}\left[\mathbf{K} L^{i \varepsilon}\right]}{\sqrt{2 \pi r}} f_{i j}^{(I)}(\theta, \varepsilon)+\frac{\operatorname{Im}\left[\mathbf{K} L^{i \varepsilon}\right]}{\sqrt{2 \pi r}} f_{i j}^{(I I)}(\theta, \varepsilon)+\frac{K_{I I I}}{\sqrt{2 \pi r}} f_{i j}^{(I I I)}(\theta)
$$


The primary change in (2.39) is the bimaterial constant and the complex stress intensity factor $(\mathbf{K})$. The complex stress intensity factor is defined and related to a dimensional form in (2.40). The value $F$ is a complex valued function and $L$ is some characteristic length such as crack length [6].

$$
\mathbf{K}=K_{I}+i K_{I I} \equiv(\text { applied stress }) \times F L^{1 / 2-i \varepsilon}
$$

The energy release rate for interfacial crack problems can now be expressed in the following equation:

$$
G=\frac{1}{E^{*}} \frac{\mathbf{K} \overline{\mathbf{K}}}{\cosh ^{2}(\pi \varepsilon)}+\frac{K_{I I I}^{2}}{2 \mu^{*}} \text { where } E^{*}=\frac{E_{1}^{\prime} E_{2}^{\prime}}{E_{1}^{\prime}+E_{2}^{\prime}}
$$

Even though $K_{I}$ and $K_{I I}$ are now coupled together in a complex form, they can still be used to represent the mode of loading at the crack face. The phase angle of loading can be determined from the following equation.

$$
\psi=\tan ^{-1}\left[\frac{\operatorname{Im}\left[\mathbf{K} L^{i \varepsilon}\right]}{\operatorname{Re}\left[\mathbf{K} L^{i \varepsilon}\right]}\right]
$$

For nonzero values of the bimaterial constant the phase angle is a function of the geometry of the problem, however as $\varepsilon \rightarrow 0$ the angle represents the strengths between the $K_{I}$ and $K_{I I}$ stress intensity factors [7].

\subsubsection{Domain Integral (Energy Release Rate)}

The domain integral is the volume form of the j-integral, which is a path independent line integral used to calculate the nonlinear energy release rate for elasticplastic systems discovered by Rice [8]. For an isotropic elastic homogenous material the $\mathrm{j}$-integral is equal to the energy release $\operatorname{rate}(G)$. This fracture parameter is calculated from the tractions, strains, and the strain energy density $(\mathbf{W})$ of the system normal to a path, $\Gamma$, as shown in Figure 2-26 and Equation (2.43).

$$
J=-\int_{\Gamma}\left(W \delta_{1 j}-\sigma_{i j} \frac{\partial u_{i}}{\partial x_{1}}\right) n_{j} d \Gamma
$$

Equation (2.43) is written in indicial notation. The strain energy density $(W)$ of the system is a scalar quantity that measures the total work per unit volume of the system and $\delta_{i j}$ is the Kronecker's delta. The second part of (2.43) consists of the stress fields $\left(\sigma_{i j}\right)$ and the spatial derivatives of the displacement $\left(u_{i}\right)$ surrounding the crack tip. $n_{j}$ is the vector normal to the path and is illustrated in Figure 2-26. 


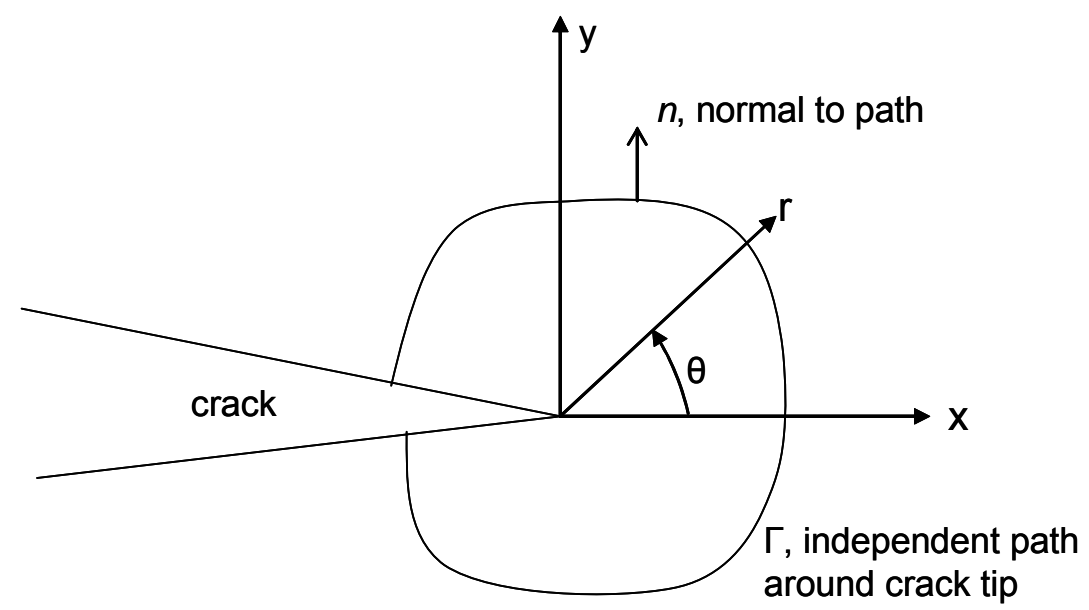

Figure 2-26: Independent path around crack tip

Use of the $\mathrm{j}$-integral for finite element applications is preferred for several reasons. Firstly, it is based on the energy change of the system, and not strictly on calculation of stress or strain fields near the crack tip. This means that the difficult problem of accurately meshing the crack tip is greatly simplified. However, while equation (2.43) is accurate for two dimensional problems, difficulties arise in calculating fracture parameters for three dimensional models. For accurate determination of energy release rates of three dimensional models, it is necessary to convert the line integral to a volume integral. A complete derivation is provided in reference $\mathrm{Li}$ et al. [9] and is summarized below.

Initially equation (2.43) is rewritten such that a closed contour $(S)$ surrounds the crack tip. This is a three dimensional volume in that the contour also includes a section of the crack length and the crack faces. Then an arbitrary test function $(\mathbf{q})$ is created such that

$$
q_{j}=\left\{\begin{array}{l}
l_{j} \text { on the crack tip } \\
0 \text { on the boundary }
\end{array},\right.
$$

where $l_{j}$ is normal to the crack tip and the test function is continuous between the crack tip and boundary.

The $\mathrm{j}$-integral can now be written as,

$$
\begin{gathered}
\bar{G}=-\oint_{\substack{\text { closed } \\
\text { contour }}} P_{j k} q_{j} n_{k} d S \\
P_{j k}=\left(W \delta_{k j}-\sigma_{i k} \frac{\partial u_{i}}{\partial x_{j}}\right) \text { is called the energy momentum tensor and consists of the }
\end{gathered}
$$

same components in (2.43). The bar over the energy release rate $(G)$ represents that this is the total energy decrease for the crack length surrounded by the contour $S$. 
Application of the divergence theorem provides:

$$
\bar{G}=-\int_{V} P_{j k} \frac{\partial q_{j}}{\partial x_{k}} d V
$$

The $V$ in the integral refers to the volume made by the closed contour. Also $\partial q_{j} / \partial x_{k}$ represents the spatial derivatives of the test function defined in (2.44).

The above calculation (2.46) assumes that the crack faces are traction-free, and thermal strains, body forces, and inertia forces are absent. For plane problems the integral is path independent, but for axisymmetric or curved fronts (2.46) is only locally path independent [10]. A complete description of this is found in the Nahta 1993 article.

The test function $\left(q_{j}\right)$ is determined by its value on the boundary and is continuous within the volume. It could be considered as the virtual crack growth. As shown in Figure 2-27, when a crack undergoes growth at a point S, a continuous function can be used to represent the growth for some arc length on the crack tip.

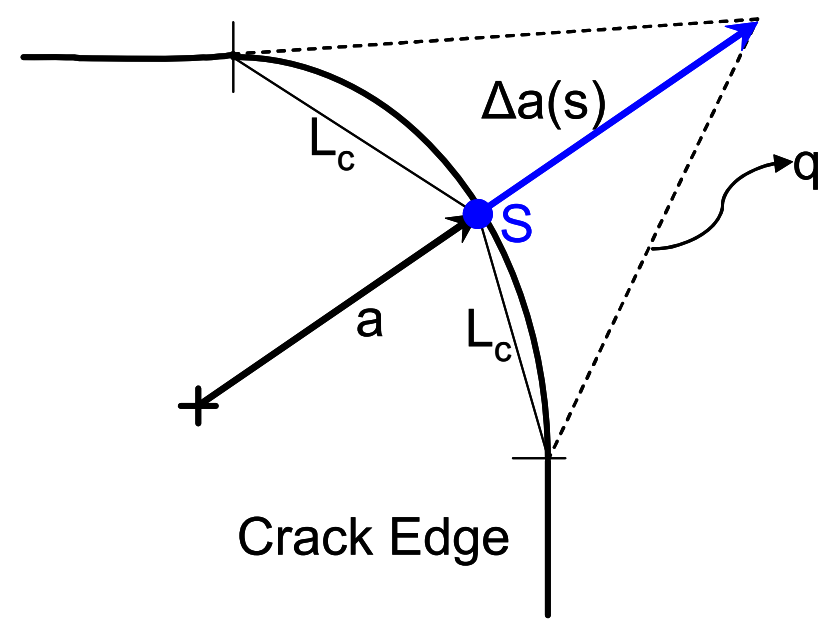

Figure 2-27: Continuous function $\mathbf{q}$ on crack tip

The pointwise value of the energy release rate assumes that for a small chord length $\left(L_{c}\right)$ the integral is the total value for the segment. If the pointwise value is considered constant along the selected chord length then division by the chord length results in the value at point $S$ as shown in equation (2.47). The unit of the pointwise value of the energy release rate $(G(s))$ is energy over area, while $\bar{G}$ has dimension of energy per length.

$$
G(s)=\frac{\bar{G}}{\int_{L_{c}} \Delta a(s) d s}
$$




\subsubsection{Thermal Domain Integral}

When thermal effects are taken into account the domain integral is no longer divergence free. In addition to mechanical strain $\left(\varepsilon_{i j}^{\text {mech }}\right)$, a thermal strain $\left(\varepsilon_{i j}^{\text {th }}\right)$ will be created from the expansion or contraction of the materials. It is now necessary to introduce a total strain which is equal to the sum of the mechanical and thermal strains.

$$
\begin{aligned}
& \varepsilon_{i j}^{\text {tot }}=\varepsilon_{i j}^{\text {mech }}+\varepsilon_{i j}^{\text {th }}, \text { where } \\
& \varepsilon_{i j}^{\text {th }}=\alpha \theta \delta_{i j}
\end{aligned}
$$

In equation (2.49) $\alpha$ is the thermal expansion coefficient, and $\theta$ is the total change in temperature.

Calculation of the strain energy density is modified to specify only mechanical strains, such that

$$
W=\int_{\varepsilon_{i j}^{\text {mech }}} \sigma_{i j} d \varepsilon_{i j}^{\text {mech }}
$$

Now when the divergence of (2.45) is taken, the effect of thermal variations must be taken into account. The final form now includes a thermal part multiplied by the test function. A detailed derivation is in the 1986 paper by Shih.

$$
\begin{aligned}
& \bar{G}=-\int_{V}\left(P_{j k} \frac{\partial q_{j}}{\partial x_{k}}+\frac{\partial P_{k j}}{\partial x_{k}} q_{j}\right) d V, \text { where } \\
& \frac{\partial P_{k j}}{\partial x_{k}}=\alpha \sigma_{i i} \frac{\partial \theta}{\partial x_{j}}
\end{aligned}
$$

\subsubsection{Interaction Integral (Stress Intensity Factors)}

The interaction integral superimposes an auxiliary solution with a known result to extract the stress intensity factors from the volume integral [11]. Initially, a total energy release rate is calculated that combines the auxiliary field values (usually a known 2-D plane strain solution) and the actual model.

$$
G_{\text {total }}=-\int_{\Gamma}\left(\frac{1}{2}\left(\sigma_{m n}+\sigma_{m n}^{a u x}\right)\left(\varepsilon_{m n}+\varepsilon_{m n}^{a u x}\right) \delta_{j k}-\left(\sigma_{i k}+\sigma_{i k}^{a u x}\right) \frac{\partial\left(u_{i}+u_{i}^{a u x}\right)}{\partial x_{j}}\right) n_{k} d \Gamma
$$

The aux superscripts denote the auxiliary stresses, strains, and displacements calculated from analytical equations. Appendix A lists the displacement equations used in calculation of plane strain interface cracks.

The interaction integral is calculated by subtracting the auxiliary and actual energy release rate. The remainder is the interaction of the actual and auxiliary components as shown in equation (2.54). The equation is simplified by using reciprocity which states $\sigma_{i j} \varepsilon_{i j}^{a u x}=\sigma_{i j}^{a u x} \varepsilon_{i j}$. 


$$
\begin{aligned}
& G_{\text {int }}=G_{\text {total }}-G-G_{\text {aux }} \\
& G_{\text {int }}=-\int_{\Gamma} P_{j k}^{\text {int }} n_{k} d \Gamma \text {, where } \\
& P_{j k}^{\mathrm{int}}=\sigma_{m n} \varepsilon_{m n}^{a u x} \delta_{j k}-\sigma_{i k} \frac{\partial u_{i}^{a u x}}{\partial x_{j}}-\sigma_{i k}^{a u x} \frac{\partial u_{i}}{\partial x_{j}}
\end{aligned}
$$

Following the same procedure as before the divergence theorem is applied to the previous equation to create a volumetric form of the interaction integral $(\bar{I})$. However, the divergence portion of the momentum tensor cannot be neglected, even asymptotically, for a curved crack front. The plane strain auxiliary field will violate compatibility for the volume and the integral will no longer be divergence free.

$$
\begin{aligned}
& \bar{I}=\bar{G}_{\text {int }}=-\int_{V}\left(P_{j k}^{\text {int }} \frac{\partial q_{j}}{\partial x_{k}}+\frac{\partial P_{k j}^{\text {int }}}{\partial x_{k}} q_{j}\right) d V \text {, where } \\
& \frac{\partial P_{k j}^{\text {int }}}{\partial x_{k}}=\left[\sigma_{m n} \varepsilon_{m n, j}^{a u x}-\sigma_{i k} u_{i, j k}^{a u x}-\sigma_{i k, k}^{a u x} u_{i, j}\right]
\end{aligned}
$$

The commas in equation (2.57) indicate a partial derivative with respect to the subscript after the comma. Equation (2.56) and (2.57) do not account for thermal strains, crack face tractions, and inertia forces.

By rewriting the first line of equation (2.54) in terms of stress intensity factors the pointwise value of the interaction integral is used to calculate the unknown stress intensity factors using equation (2.58). The specific stress intensity can be found by setting the corresponding auxiliary value equal to one and letting the other values equal zero, for instance the calculation of $K_{I}$ is done by setting $K_{I}=1$ and $K_{I I}=K_{I I I}=0$ in equation (2.59).

$$
\begin{aligned}
& I(s)=\frac{2}{E^{*} \cosh ^{2}(\pi \varepsilon)}\left[K_{I} K_{I}^{a u x}+K_{I I} K_{I I}^{a u x}\right]+\frac{1}{\mu^{*}} K_{I I I} K_{I I I}^{a u x} \\
& K_{I}=\frac{I(s)}{2} E^{*} \cosh ^{2}(\pi \varepsilon)
\end{aligned}
$$

\subsubsection{Thermal Interaction Integral}

Previously, the stress intensity factor was calculated using the interaction integral as described in the 1998 Gosz paper. While that form of the integral can analyze curvilinear bimaterial cracks the integral does not account for thermal strains. The interaction integral was reexamined to include thermal effects in the derivation. The newly derived equation is shown below.

$$
\bar{I}=\bar{G}_{\mathrm{int}}=-\int_{V}\left(P_{j k}^{\mathrm{int}} \frac{\partial q_{j}}{\partial x_{k}}+\frac{\partial P_{k j}^{\mathrm{int}}}{\partial x_{j}} q_{k}\right) d V
$$




$$
\begin{aligned}
& P_{j k}^{\mathrm{int}}=\sigma_{m n} \varepsilon_{m n}^{a u x} \delta_{j k}-\sigma_{i k} \frac{\partial u_{i}^{a u x}}{\partial x_{j}}-\sigma_{i k}^{a u x} \frac{\partial u_{i}}{\partial x_{j}} \\
& \frac{P_{k j}^{\mathrm{int}}}{\partial x_{j}}=\sigma_{i j} \varepsilon_{i j, k}^{a u x}-\sigma_{i j} u_{j, i k}^{a u x}-\sigma_{i j, i}^{a u x} u_{j, k}-\alpha \sigma_{i i}^{a u x} \theta_{, k}
\end{aligned}
$$

The last term in equation (2.62) is the only new term in the interaction integral, and multiplies the thermal expansion coefficient and the trace of the stress by the spatial derivatives of the temperature. The integral assumes zero body forces and crack face tractions, but can be used for curvilinear cracks. The material is also isotropic.

\subsubsection{Finite Element Implementation}

Since most commercial FEM packages have limited or no domain integral capabilites it is more convenient to create a program to calculate the fracture parameters during postprocessing. For the purposes of this study the finite element programs were performed using the software ANSYS 7.1 with the postprocessing of fracture parameters performed using the commercial programming language Matlab 6.5. Development of the postprocessing procedure was based on the procedures described from reference [9] and [12]. Figure 2-29 is a flow chart representing the steps taken during postprocessing.

\section{Post Processing of Domain Integral}

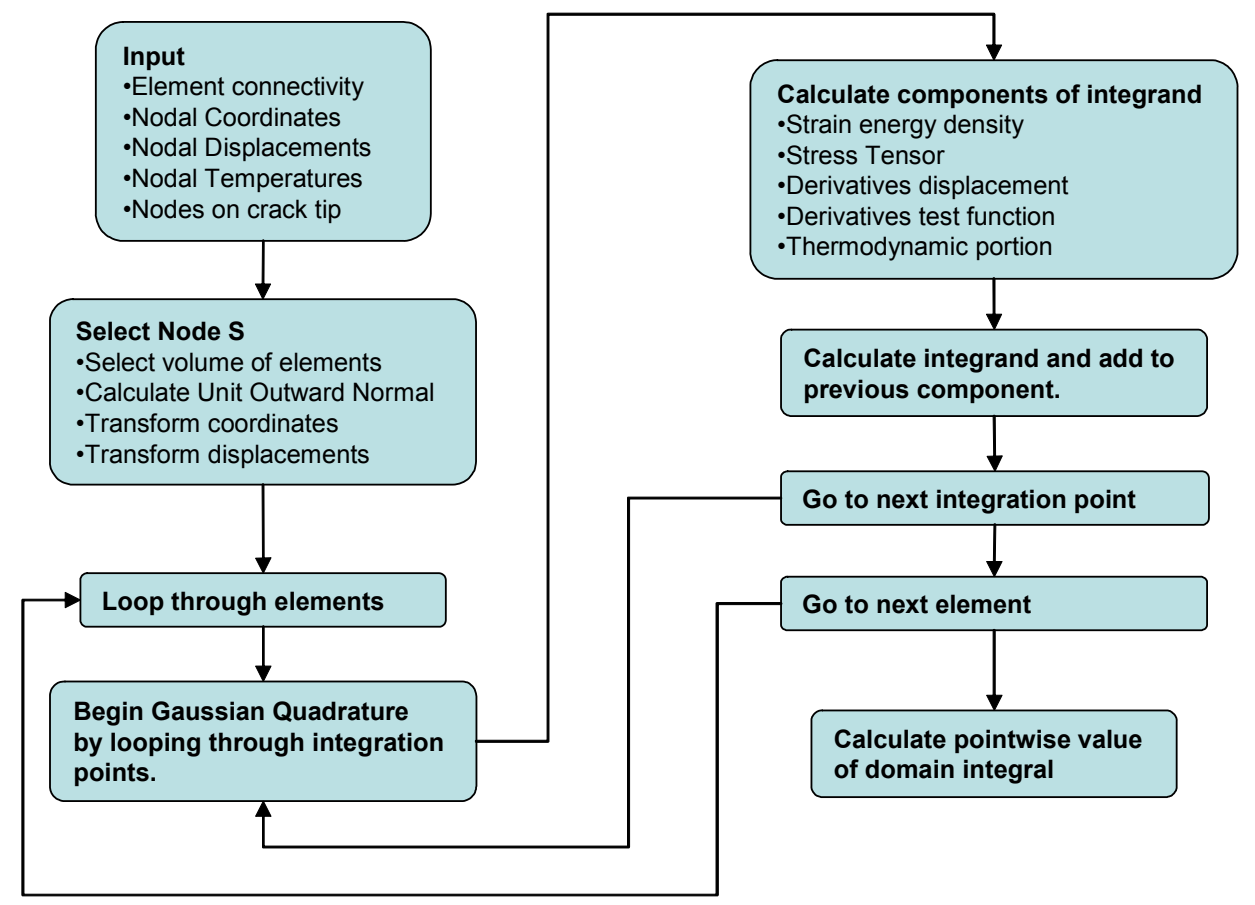

Figure 2-28: Flow chart of steps taken during postprocessing of domain integral

The calculation of the interaction integral can be performed concurrently with the calculation of the energy release rate, but several additional calculations are required. In 
his 1998 paper Gosz describes the creation of a curvilinear coordinate system to eliminate the derivatives with respect to the out of plane component of the auxiliary plane strain solution. A new coordinate system is created for each integration point, which greatly simplifies calculation of the higher order gradients. A detailed description of this process is included in Appendix B. The appendix includes the steps necessary to calculate the higher order gradients that occur in the interaction integral. The additional steps necessary to calculate the interaction integral are listed Table 2-8 and start at the first Gaussian integration point (refer Figure 2-28):

Table 2-8: Additional postprocessing steps for computation of interaction integral

1. Calculate Point $\mathrm{S}$ on the crack tip closet to the integration point.

Set Point $\mathrm{S}$ as the new origin and transform coordinates, displacements,

2. and test function to new system. (The crack plane unit vectors will be normal and tangent to the crack curve at Point S.)

3. Calculate the radius of curvature of the crack edge at point $\mathrm{S}$.

4. Calculate auxiliary values of interaction integral using methods described

4. in appendix A.

5. Input auxiliary values and finite element values into equation (2.56).

6. Continue to next integration point.

\subsubsection{Finite Element Calculations}

Calculation of the volume integrals in Section 2.6.2 can be done using several basic concepts and various constants concerning calculation of stress and strain from finite element methods. Gaussian quadrature is the numerical integration process used for the post processing code. The following paragraphs briefly discuss the steps taken to calculate the domain integral (2.46).

The strain energy density of each element is calculated by dividing the total strain energy of the element by the element's volume. The strain energy $(U)$ is calculated from equation (2.63) where $\mathbf{E}$ is the elasticity matrix. The subscript $(e)$ denotes the value for each element, while $V$ is the volume of the selected element.

$$
U^{(e)}=\int_{V} \frac{1}{2} \varepsilon^{T} \mathbf{E} \varepsilon d V
$$

The second part of the momentum tensor $(\mathbf{P})$ looks at the stress and strain fields within the volume. Using the chain rule the derivatives of the displacements $\left(u_{i}\right)$ can be 
calculated using equation (2.64), where $N_{I}$ is the shape functions for the element and $r_{J}$ represents the natural coordinates.

$$
\frac{\partial u_{i}}{\partial x_{j}}=\sum_{I=1}^{\text {node }} \sum_{J=1}^{3} \frac{\partial N_{I}}{\partial r_{J}} \frac{\partial r_{J}}{\partial x_{j}} u_{i I}
$$

Lastly the test function ( $\mathbf{q}$ ) needs to be fully defined. If the node of interest (S), is taken to be the new origin and the nodal coordinates and transformations are translated as shown in Figure 2-29 the definition of $\mathbf{q}$ is simplified. The nodal values of $\mathbf{q}$ is defined by a piecewise function. Nodal values on the outer boundary are equal to zero while the nodal values within the volume are represented by any continuous vector. Therefore the nodal $(Q)$ value at each node can be defined below:

$$
Q_{I}=\left\{\begin{array}{l}
{\left[\begin{array}{lll}
1 & 0 & 0
\end{array}\right] \text { within volume }} \\
0 \text { on outer boundaries }
\end{array}\right.
$$

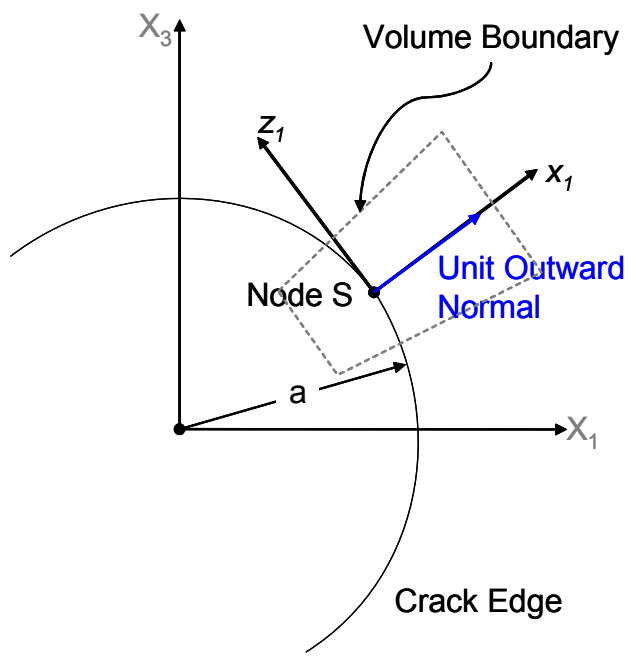

Figure 2-29: Transformation to new coordinate system and nodal Q values

By defining the test function at each node, the isoparametric formulation can be used to calculate the derivatives at each integration point.

$$
\frac{\partial q_{i}}{\partial x_{j}}=\sum_{I=1}^{\text {node }} \sum_{J=1}^{3} \frac{\partial N_{I}}{\partial r_{J}} \frac{\partial r_{J}}{\partial x_{j}} Q_{i I}
$$

It is now possible to calculate the average domain integral and the energy release rate at node $\mathrm{S}$ using the Gaussian Quadrature as shown below. 


$$
\bar{G}=-\sum_{e-V}\left\{\sum_{k=1}^{n} \sum_{l=1}^{n} \sum_{m=1}^{n}(\operatorname{tr}[W \mathbf{I}-\nabla \mathbf{u} \cdot \sigma] \nabla \mathbf{q}-(\alpha \theta \mathbf{I}) \mathbf{q}) \operatorname{det} \mathbf{J} w_{k} w_{l} w_{m}\right\}
$$

Equation (2.67) sums all the elements $(e)$ that appear within the selected volume $(V)$ and is written in tensorial notation. The number of Gaussian points used is designated by $n$ and the Gaussian weighting values are designated by $w$. The differential volume $(d V)$ is represented by the determinant of the Jacobian matrix $(\mathbf{J})$. The Jacobian matrix and is defined below.

$$
d V=\operatorname{det}(J)=\operatorname{det}\left(\frac{\partial x_{i}}{\partial x_{j}}\right)
$$

Before calculating the pointwise value the chord lengths between nodes $\mathrm{S}$ and the nodes $S+1$ and $S-1$ on the crack tip need to be defined as $L_{1}$ and $L_{2}$ (refer to Figure 2-27). If the values are know then:

$$
G(s)=\frac{2 \bar{G}}{L_{1}+L_{2}}
$$

\subsubsection{Program Summary}

For this method of calculation the meshing and volume selection for the domain integral have requirements set by code limitations. A mapped mesh was used to insure that selected nodes were always normal to the crack plane and to simplify definition of the test function $(\mathbf{q})$. Secondly, while the size of the volume is flexible perpendicular and normal to the crack only one row of elements can be included on either side of node $\mathrm{S}$. This is necessary for more accurate calculation of the pointwise value.

Table 2-9 summarizes the capabilities of the post processing MatLab code created. It incorporates the thermal and curvilinear coordinates in the interaction integral to allow for a flexible postprocessing routine.

Table 2-9: Summary of post-processing capabilities.

\begin{tabular}{|c|l|}
\hline \multirow{3}{*}{ Model Types } & -In-plane curvilinear 3d cracks \\
& $\begin{array}{l}\text {-Homogenous cracks } \\
\text {-Bimaterial Interface cracks } \\
\text {-Thermally Stressed }\end{array}$ \\
\hline \multirow{2}{*}{ Programming } & -20 Node brick elements \\
Limitations & $\begin{array}{l}\text {-Mesh normal from crack edge } \\
\text {-Zero body forces } \\
\end{array}$ \\
\hline -Zero crack face tractions \\
\hline Outputs & -Energy Release Rate $(G)$ \\
\hline
\end{tabular}




\begin{tabular}{|l|l|}
\hline & $\begin{array}{l}\text {-Mode I, II, and III stress intensity } \\
\text { factors }\left(K_{I}, K_{I I}, \text { and } K_{I I I}\right)\end{array}$ \\
-Phase Angle $(\psi)$
\end{tabular}

\subsection{FMA Verification: Axisymmetric Bimaterial and Thermally Stressed Cracks}

If the domain and interaction integrals are to be of significance it is necessary to look at fracture parameters at all points on the crack edge. This is especially important with regard to fracture within fuel cells where the electrochemical reactions cause varied stress fields. Initially a standard model of a penny shaped crack was created. This model was used to verify the accuracy of the domain integral calculations under a known solution. Eventually, this same model will be used to analyze fracture in a fuel cell using the global-local modeling techniques.

\subsubsection{Bimaterial Penny Shaped Crack}

A crack was considered to occur between the anode and electrolyte; refer to Table 2-5 for the material constants used. The solution for the problem is in equation (2.70) and was taken from [13].

$$
K_{I}+i K_{I I}=2 \sigma_{o} \sqrt{a} \frac{\Gamma(2+i \varepsilon)}{\Gamma(.5+i \varepsilon)}(2 a)^{-i \varepsilon}
$$

$\sigma_{o}$ is the remote stress applied to the model. The gamma function $(\Gamma)$ can be defined from the power series as shown below [14].

$$
\Gamma(x)=\lim _{n \rightarrow \infty} \frac{n ! n^{x-1}}{x(x+1)(x+2) \ldots(x+n-1)}, \text { for any real or complex } x
$$

The fracture model was developed and analyzed with Ansys 7.0 software and used the 20 node brick element. The crack tip mesh used only brick elements to reduce computation and to simplify meshing of future crack configurations. For a circular mesh the element size (e) along the crack tip is held at a constant ratio with respect to the crack length (a). This element size was held constant for nine layers extending in all four normal directions from the crack tip and crack plane. Outside the layers around the crack tip the element size is allowed to increase as shown in Figure 2-30. Since one-quarter symmetry was used the planar sides were considered to be on rollers. A remote pressure was applied to the top and bottom, while the model was held fixed in the vertical direction at the bottom. 


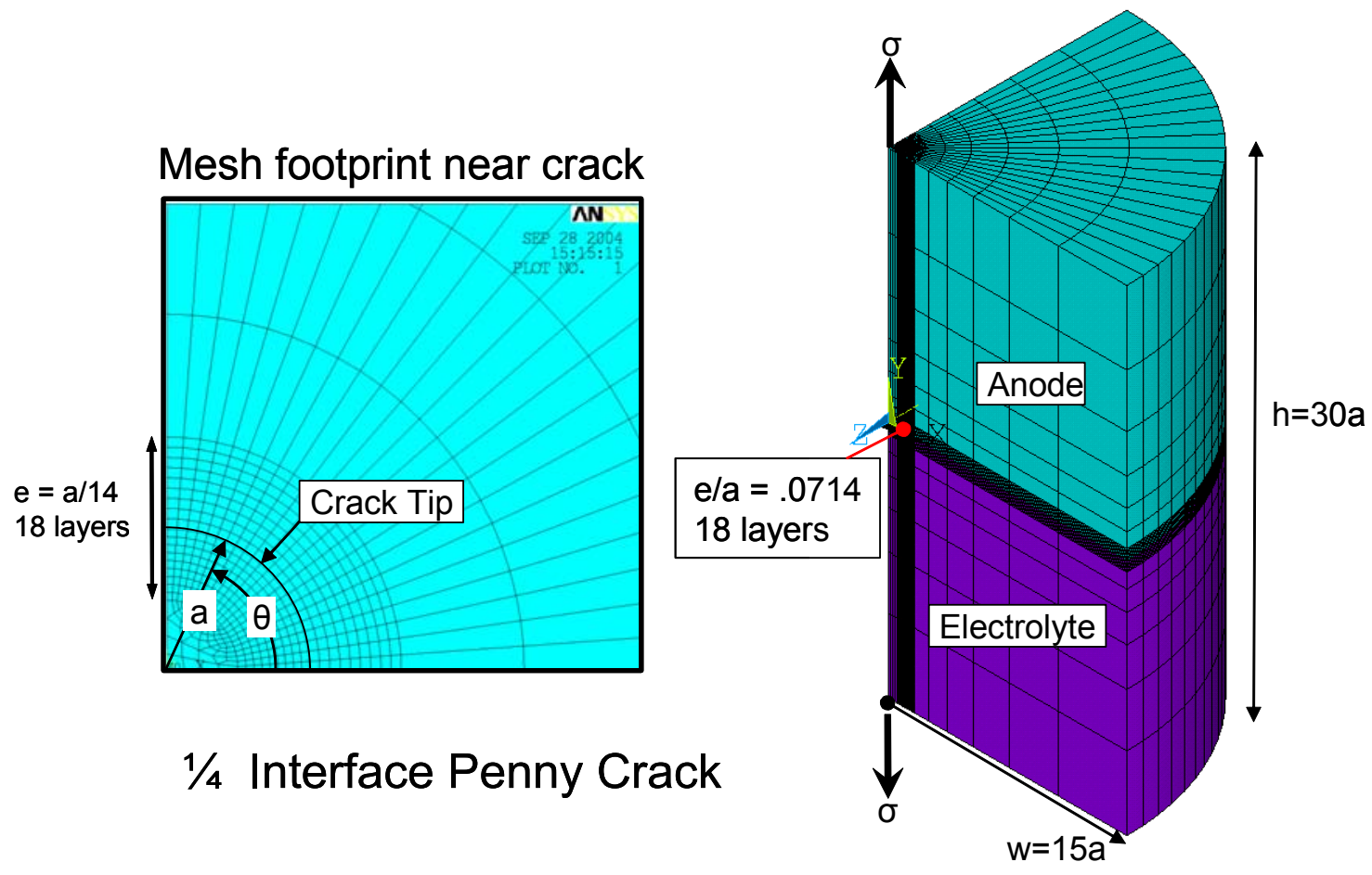

Figure 2-30: Model configuration and boundary conditions for bimaterial penny crack

Several sets of data were calculated from the crack model. The values of the complex stress intensity factors, the energy release rate calculated by both the domain integral and from the stress intensity factors using the stress intensity factors, and finally the value the phase angle.

In Table 2-10 normalized values of the fracture parameters are compared between the analytical result (6.11) and the numerical results obtained in this thesis. The table lists values taken at the maximum location of error. It can be seen that although there is error in both KI and KII, the larger error in KII dominants the results of the phase angle. It can also be seen that the energy release rate when calculated from the numerical stress intensity factors is much more accurate than the energy release rate from the domain integral. This is reasonable since the domain integral calculation does not account for the nonzero divergence of the curvilinear crack.

Table 2-10: Normalized Fracture Parameters at Theta $\sim 90^{\circ}$ (Location of Max Error)

\begin{tabular}{|c|c|c|c|c|c|}
\hline $\begin{array}{c}\text { Crack } \\
\text { Size }(\boldsymbol{\mu m})\end{array}$ & $\frac{\sqrt{\pi} \operatorname{Re}\left[\mathbf{K}(2 a)^{i \varepsilon}\right]}{2 \sigma_{o} \sqrt{a}}$ & $\frac{\sqrt{\pi} \operatorname{Im}\left[\mathbf{K}(2 a)^{i \varepsilon}\right]}{2 \sigma_{o} \sqrt{a}}$ & $\frac{\pi E^{*} \cosh ^{2}(\pi \varepsilon) G}{4 \sigma_{o} a}$ & $\frac{\pi}{4 \sigma_{o} a} \mathbf{K} \overline{\mathbf{K}}$ & $\left.\boldsymbol{\psi} \mathbf{(}^{\mathbf{o}}\right)$ \\
\hline Numerical & 0.992 & 0.074 & 1.077 & 0.989 & 3.00 \\
\hline Analytical & 0.986 & 0.077 & 0.996 & 0.996 & 3.12 \\
\hline
\end{tabular}




\begin{tabular}{|l|l|l|l|l|l|}
\hline$\%$ Error & -.629 & 3.390 & -8.166 & 0.990 & 3.71 \\
\hline
\end{tabular}

For prediction of crack growth in fuel cell models an understanding of error magnitude with respect to mesh location also needs to be developed. Figure 2-31 shows the angle for each point along the crack tip. A plot of the normalized values of KI and KII at each node of the crack tip and a plot of the magnitude of error for KI, KII and the phase angle are shown in Figure 2-32 through Figure 2-34 with respect to the angle of Figure 2-31. In the case of Figure 2-34 it can be seen how the error of KI and KII help magnify error for the phase angle calculation.

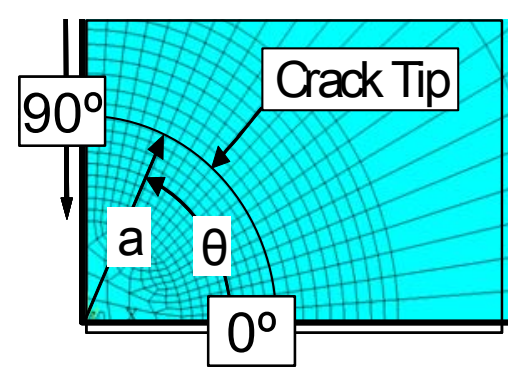

Figure 2-31: Angle coordinates along crack tip

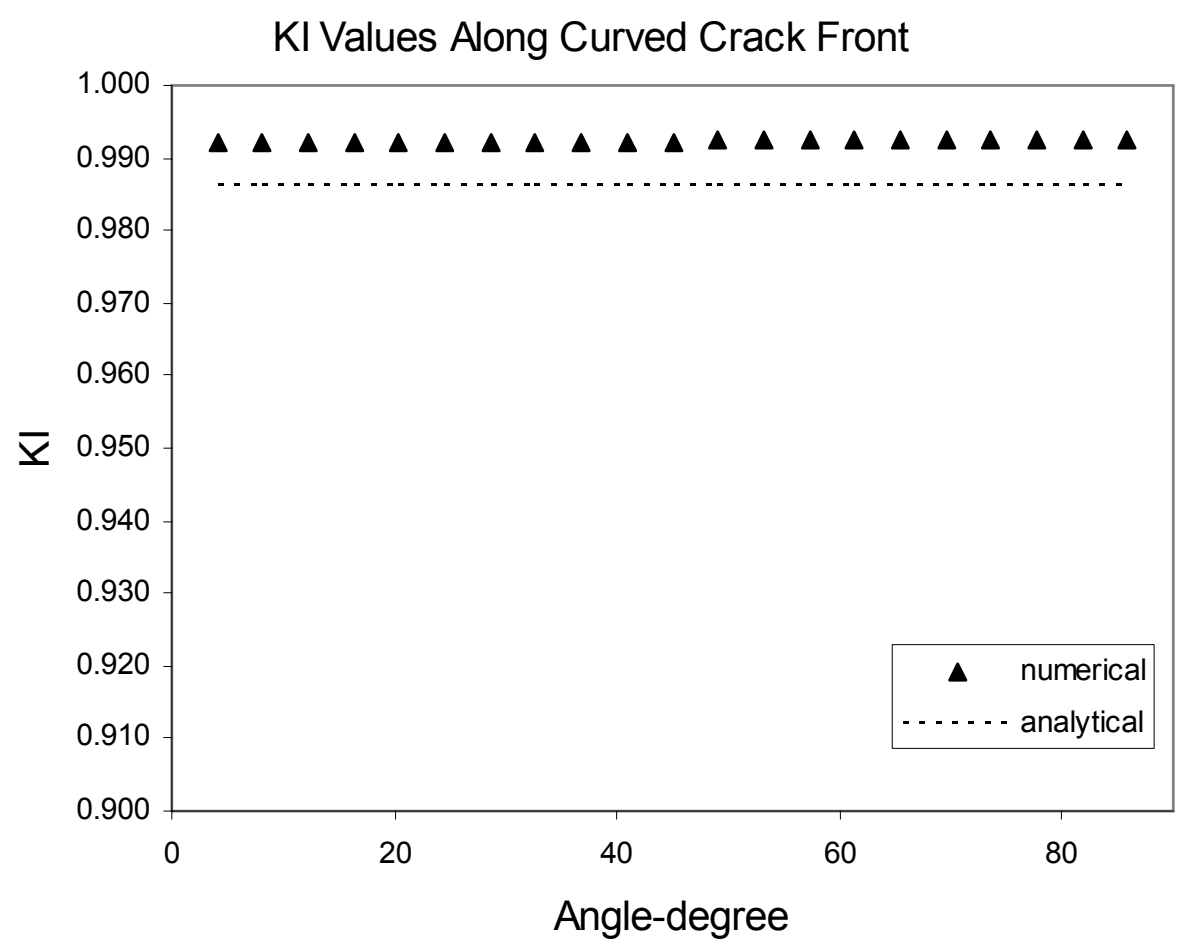

Figure 2-32: Normalized Values of KI with respect to angle 


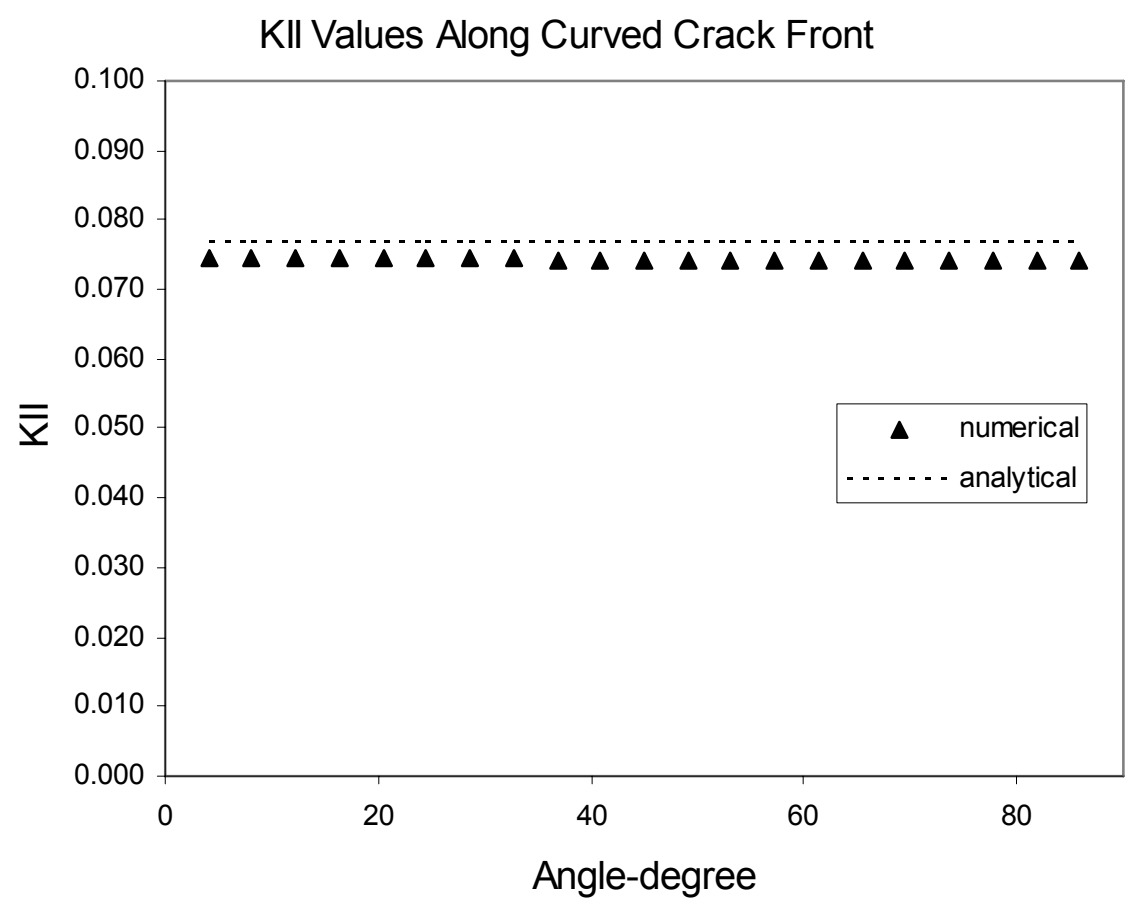

Figure 2-33: Normalized Values of KII with respect to angle

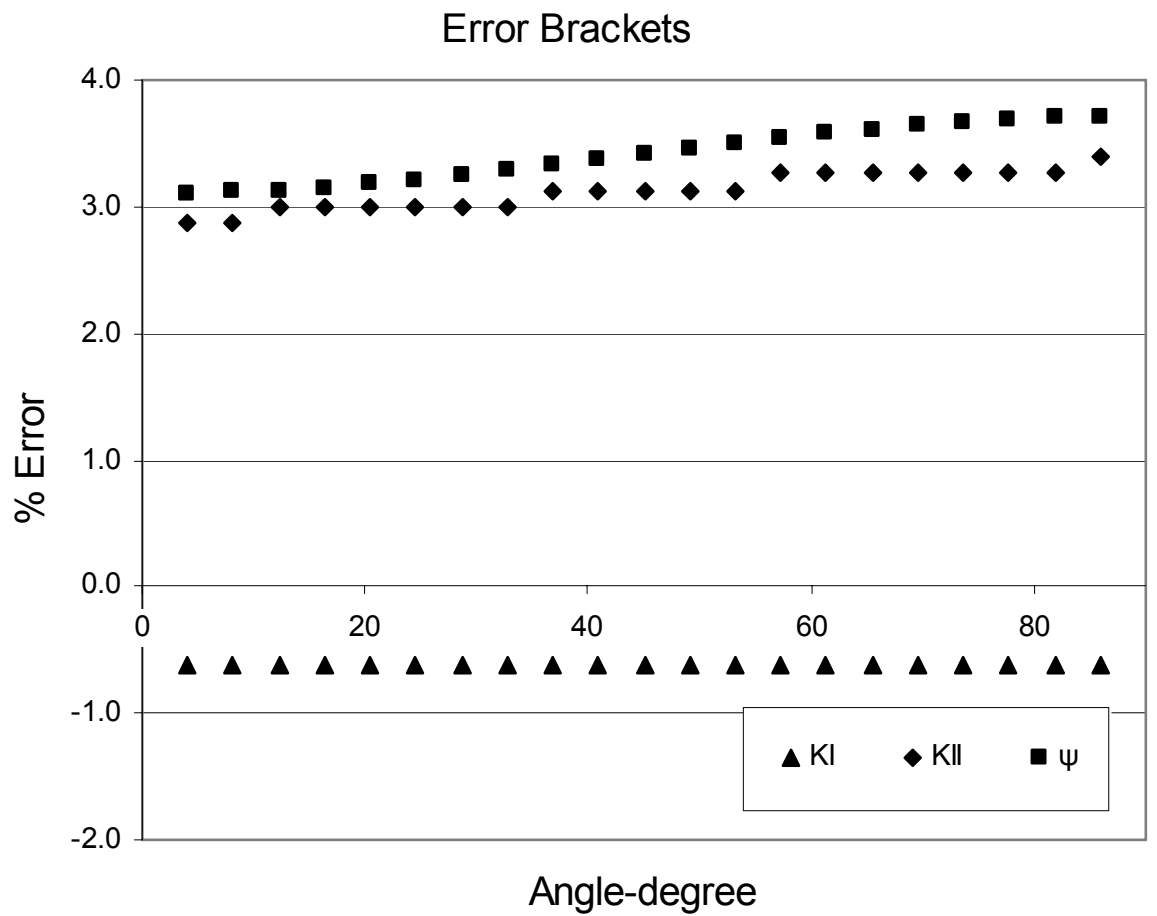

Figure 2-34: \% Error along curved crack front 


\subsubsection{Thermally Stressed Straight Crack}

A straight crack was used to examine the accuracy of the interaction integral because of the availability of a known solution. The fracture parameters of this model can be compared to an isothermal model in which the crack face is loaded by tractions equal to those that appear on a non-cracked model under the same thermal load [15].

The solution was verified by two different methods. First it was compared to the FEM solution of the 2-D plane strain crack loaded with crack face tractions. An analytical solution for the crack traction loading in an infinite body is also examined, refer to Tada's The Stress Analysis of Cracks Handbook [14]. The analytical solution of a crack face under a linear stress distribution is shown in equation (2.72). The pressure $(p)$ is the maximum traction on the crack face and is calculated from the maximum temperature $\left(\theta_{f}\right)$ and thermal expansion coefficient $(\alpha)$ in equation (2.73).

$$
\begin{aligned}
& K_{I}=\left(1-\frac{2}{\pi}\right) p \sqrt{\pi a} \\
& p=\frac{E \alpha \theta_{f}}{1-v}
\end{aligned}
$$

Figure 2-35 shows the material properties used and the model configuration. One-quarter symmetry is applied such that the symmetry planes lie on the crack plane and along the xy-plane. Finite element boundary conditions placed these planes on rollers. The external displacement conditions placed the top edge on rollers with one corner fixed. The crack length to width ratio $(a / w)$ is set to 0.5 , while the height to width ratio $(h / w)$ is 2.0 . The model thickness is equal to one-half the width. The entire model was meshed with 20 node brick elements with a 1:1 aspect ratio and a characteristic length of $a / 10$. The final boundary condition applied is the temperature variation. The stress free temperature was set to zero degrees. The linear temperature condition is proportional to the $\mathrm{x}$-coordinate and equals zero at the origin. The maximum and minimum temperatures occurred at the outer edges. 


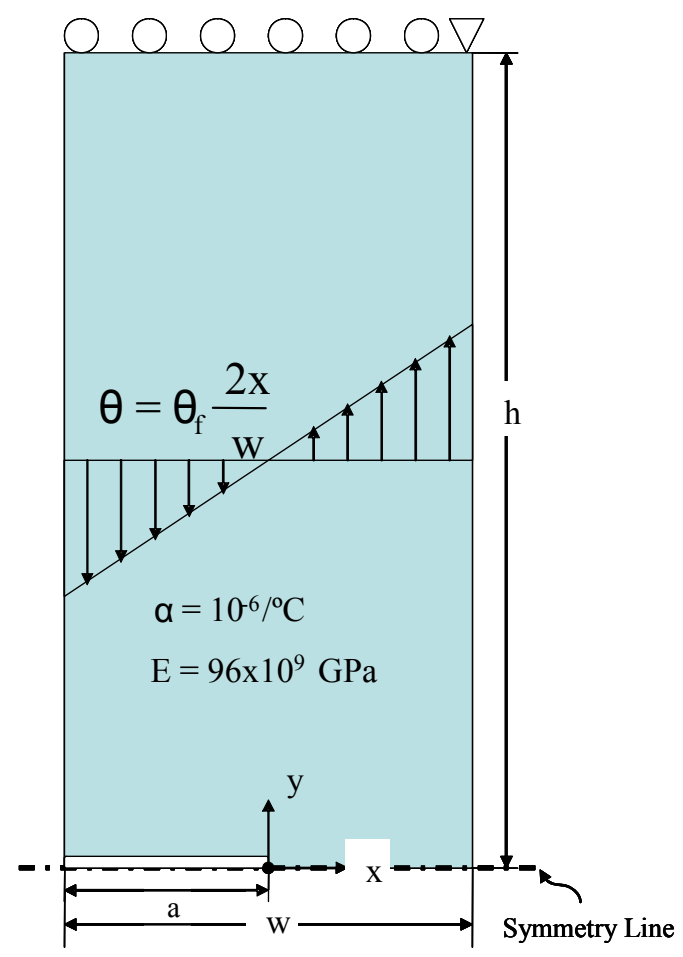

Figure 2-35: Thermally stressed crack model

The stress intensity factors should be independent of the domain of the volume integral. To verify this, the stress intensity factors were calculated for increasing integration volumes using equation (2.60). Only one point along the width of the crack was examined; at the center of the body (the mid-width symmetry line). The values were calculated at this point to maximize plane strain conditions for comparison to the two dimensional analytical solution. The normalized values for each volume are shown in Table 2-11. Each volume is designated by the number of elements selected for analysis and the stress intensity values are normalized by the maximum pressure seen in the body.

Table 2-11: Normalized values of KI stress intensity

\begin{tabular}{|c|c|c|}
\hline \# Elements & $\frac{K_{I}}{p(\pi a)^{1 / 2}}$ & $\frac{K_{I}}{p(\pi a)^{1 / 2}}$ \\
in domain & from FEM & from eqn. (2.72) \\
\hline 4 & .3615 & \multirow{2}{*}{.36} \\
\hline 16 & .3651 & .3630 \\
\hline 36 & .3651 & \\
\hline 70 & .3651 & \\
\hline
\end{tabular}




\subsubsection{FMA using MARC FEM Commercial Software.}

The Fracture Mechanical Analyzer (FMA) program was combined with MARC software to calculate the stress intensity factors for bimaterial axisymmetric cracks. The program is also used to calculate these values at multiple locations along the crack front. The following numerical example demonstrates the FMA/MARC capability of calculating the complex stress intensity factor. This is of importance in studying fuel cells, due to the bonded structure of the PEN layers and seals.

The numerical example shown here consists of a penny shaped crack placed within a large cylinder. The upper half of the cylinder is the electrolyte material and the bottom half is the anode material. The material properties used were a Young's Modulus of $200 \mathrm{GPa}$ for the YSZ electrolyte, while the Ni-YSZ anode has a Modulus of $96 \mathrm{GPa}$. Both materials had a Poisson's ratio of 0.3. The materials are considered to be perfectly bonded except at the location of a circular crack at the center of the model. The diameter and total height of the cylinder were large enough so that the crack can be considered to be in an infinite body and boundary effects are neglected. The cylinder was placed under uniform tension, normal to the crack plane. The model will be analyzed using MARC software and then the FMA program will be used to calculate an important fracture parameter, i.e. the complex stress intensity factor.

FEM Model: The fracture model was imported from a previous ANSYS fracture model and used 20 node brick elements. The crack tip mesh used only brick elements to reduce computation and to simplify meshing of future crack configurations. For a circular mesh the element size (e) along the crack tip is held at a constant ratio with respect to the crack length (a). For this model the ratio of crack size to element size was 8:1. This element size was held constant for seven layers extending in all four normal directions from the crack tip and crack plane. Outside the layers surrounding the crack tip, the element size is allowed to increase as shown in Figure 2-36. Since one-quarter symmetry was used the planar sides were considered to be on rollers. A uniform pressure of $100 \mathrm{MPa}$ was applied to the cylinder top to create the remote pressure applied to normal to the crack plane. The positive y-axis is the electrolyte material. 


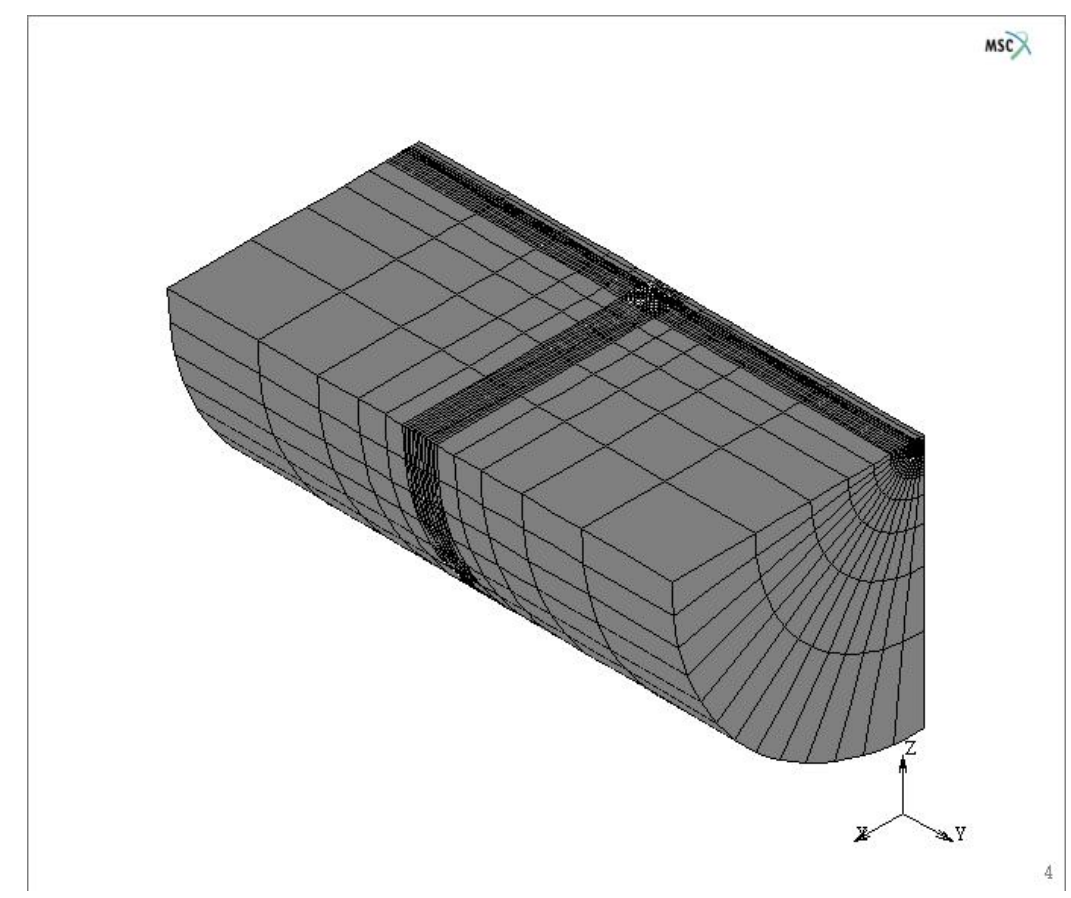

Figure 2-36: Fracture Model (Penny Shaped Crack with 1/4 Symmetry)

Results: The analytical solution for the MARC model is the listed in equation(2.70). The analytical solution for the combined MARC/FMA model is listed in Table 2-12. Figure 2-37 and Figure 2-38 plot the components of the stress intensity factor along the crack front. The $\mathrm{K}_{\mathrm{II}}$ value is negative for this model due to the location of the electrolyte; if the materials were reversed $\mathrm{K}_{\mathrm{II}}$ would be positive. Figure 2-39 plots the error variation along the crack front. Contour plots of the model can be found in Figure 2-40 and Figure 2-41.

Table 2-12: Analytical Results for Combined MARC and FMA analysis

\begin{tabular}{|c|c|}
\hline Applied Stress $\left(\sigma_{\mathrm{o}}\right)$ & $10 \mathrm{MPa}$ \\
\hline Crack Size $(\mathrm{a})$ & $.1 \mathrm{~m}$ \\
\hline Material Mismatch $(\varepsilon)$ & .0321 \\
\hline Marc Result for $\mathrm{K}_{\mathrm{I}}$ & $35.260 \mathrm{MPa} \bigvee_{\mathrm{m}}$ \\
\hline Marc Result for $\mathrm{K}_{\mathrm{II}}$ & $4.535 \mathrm{MPa} \bigvee_{\mathrm{m}}$ \\
\hline
\end{tabular}




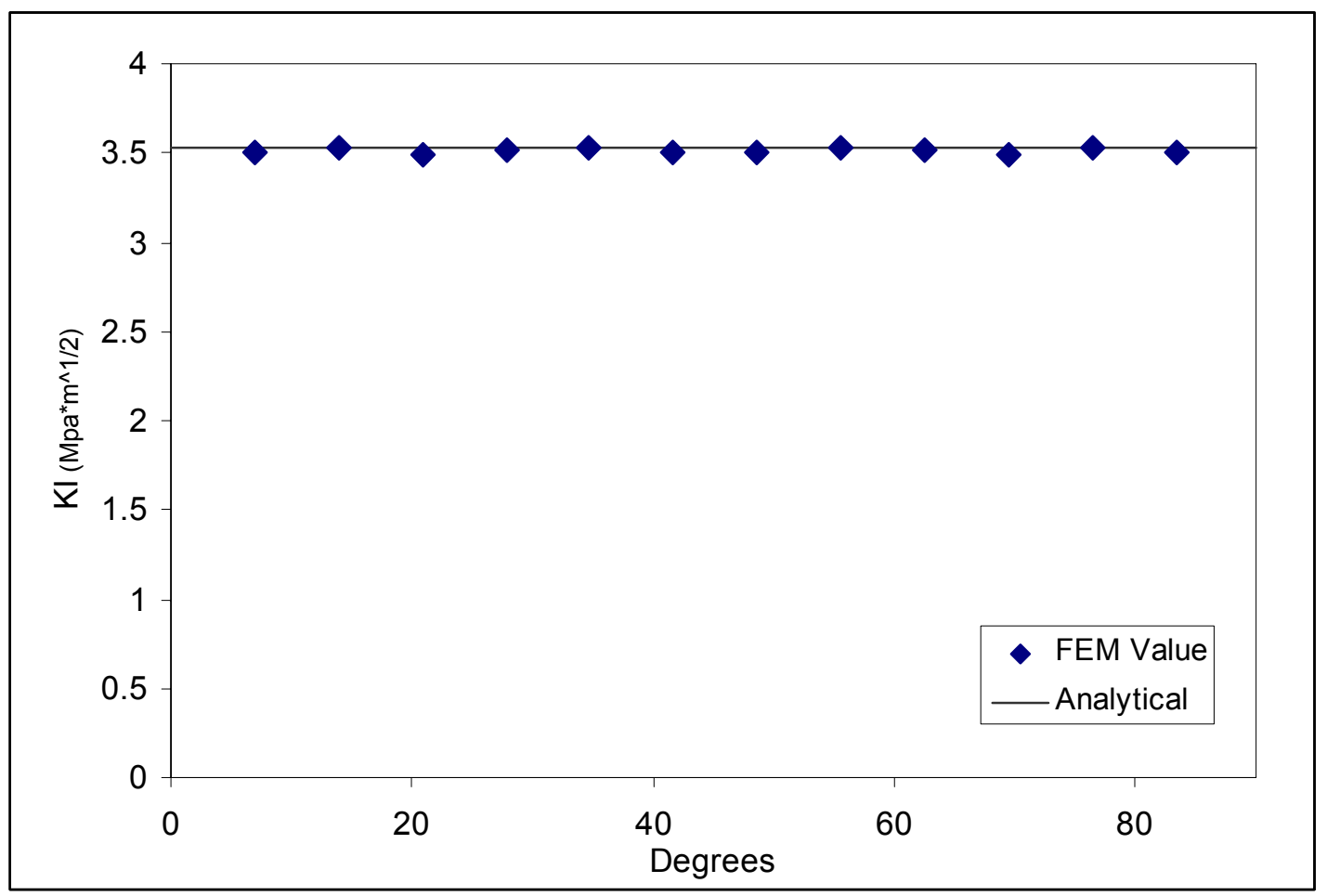

Figure 2-37: Variation of calculated $K_{I}$ values along crack tip

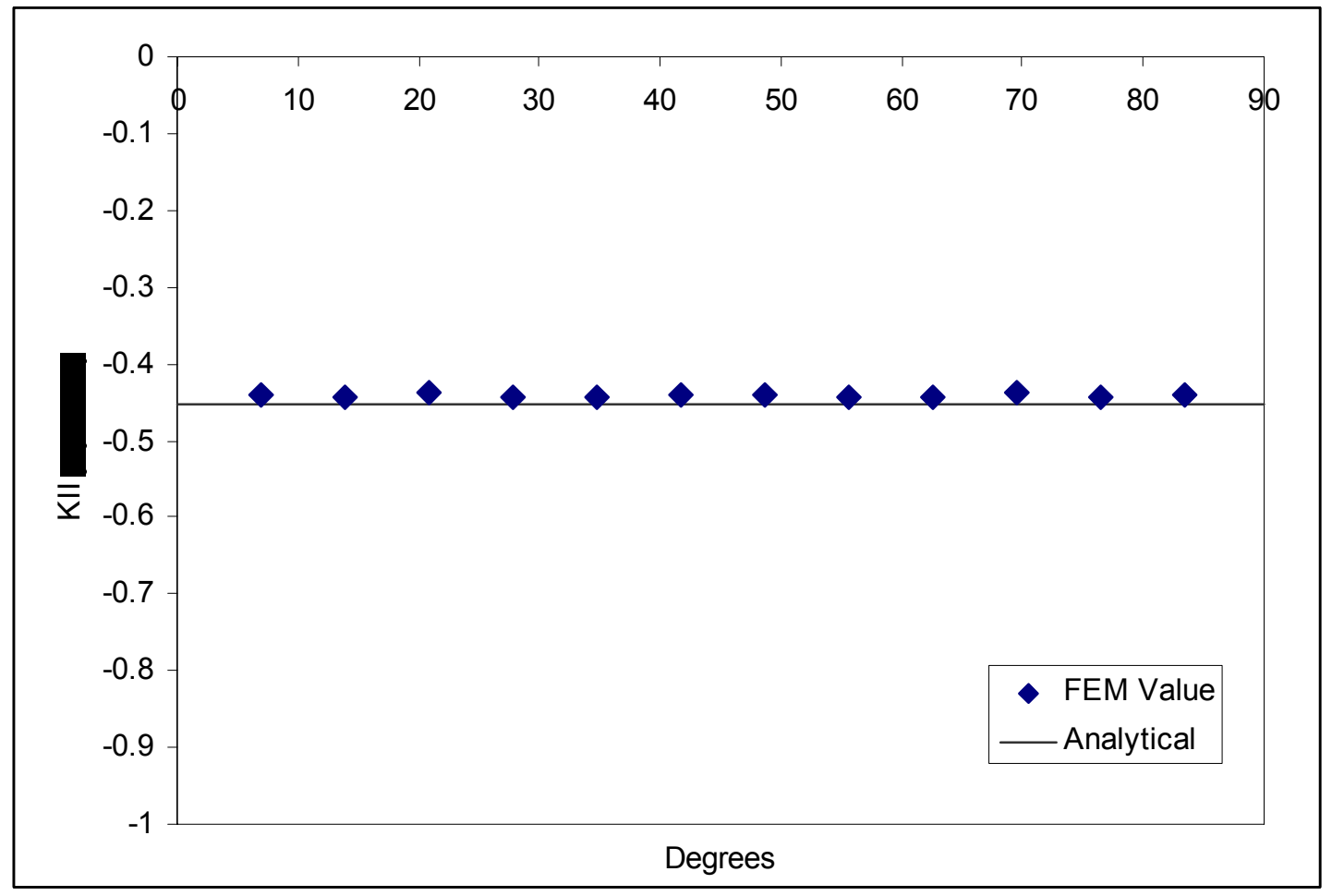

Figure 2-38: Variation of calculated $\mathrm{K}_{\mathrm{II}}$ values along crack tip 


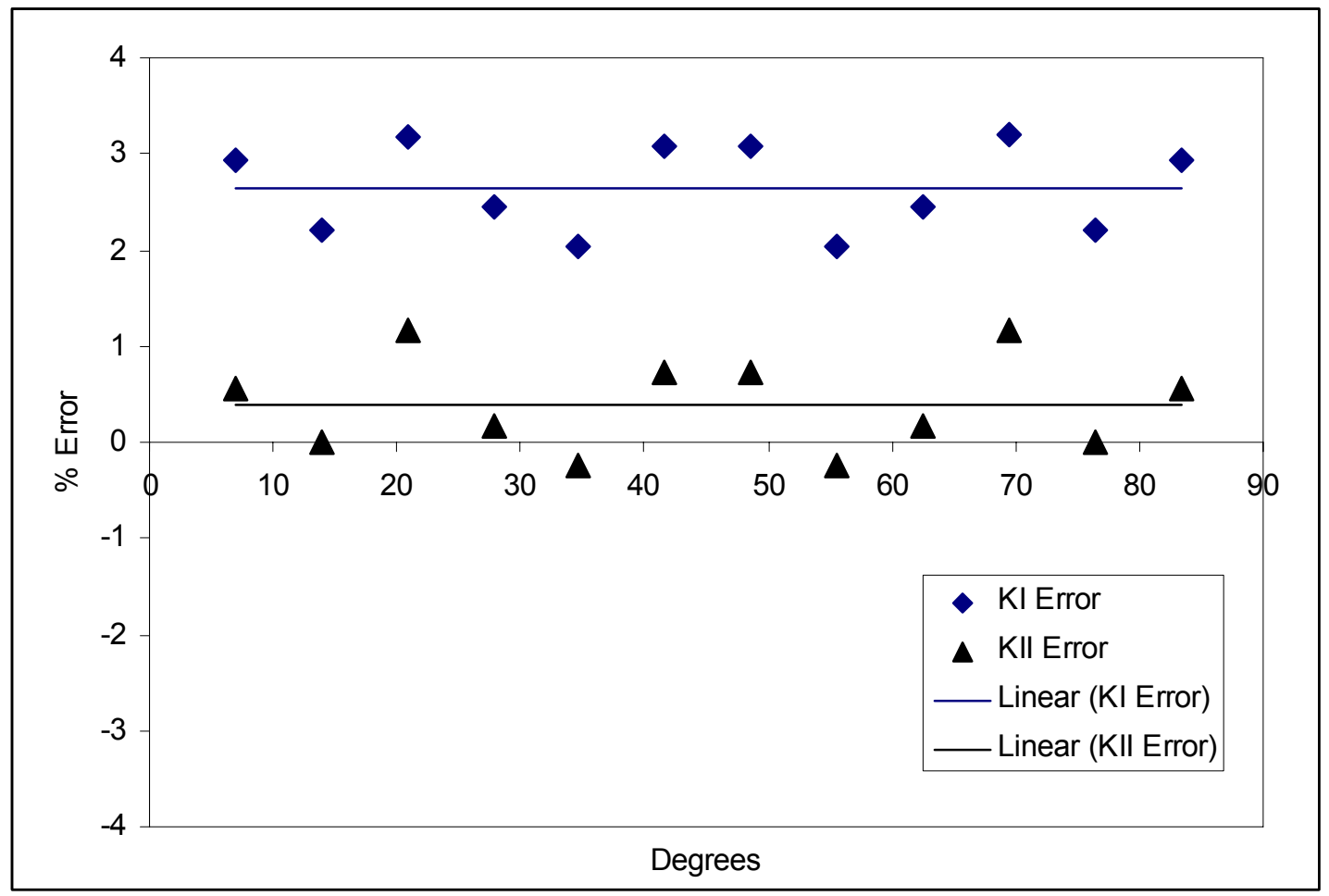

Figure 2-39: Variation of percent error along crack tip

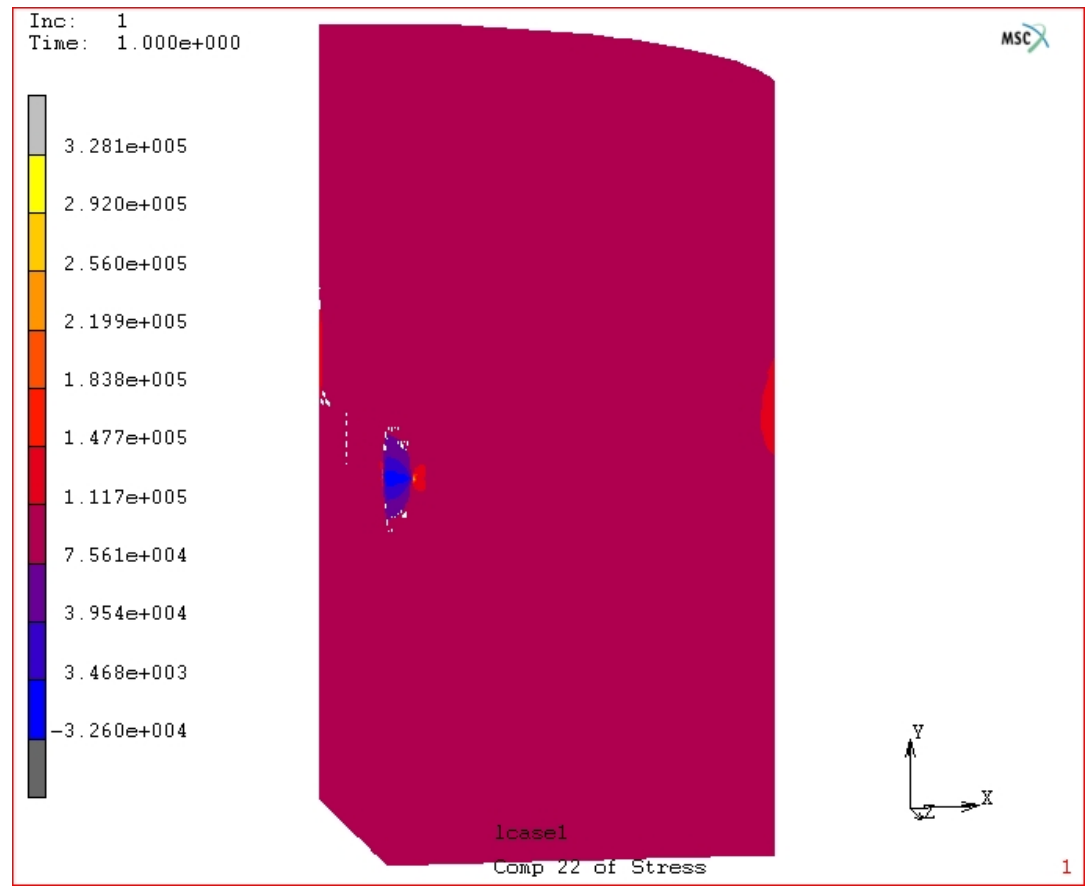

Figure 2-40: Sigma-yy stresses for entire model 


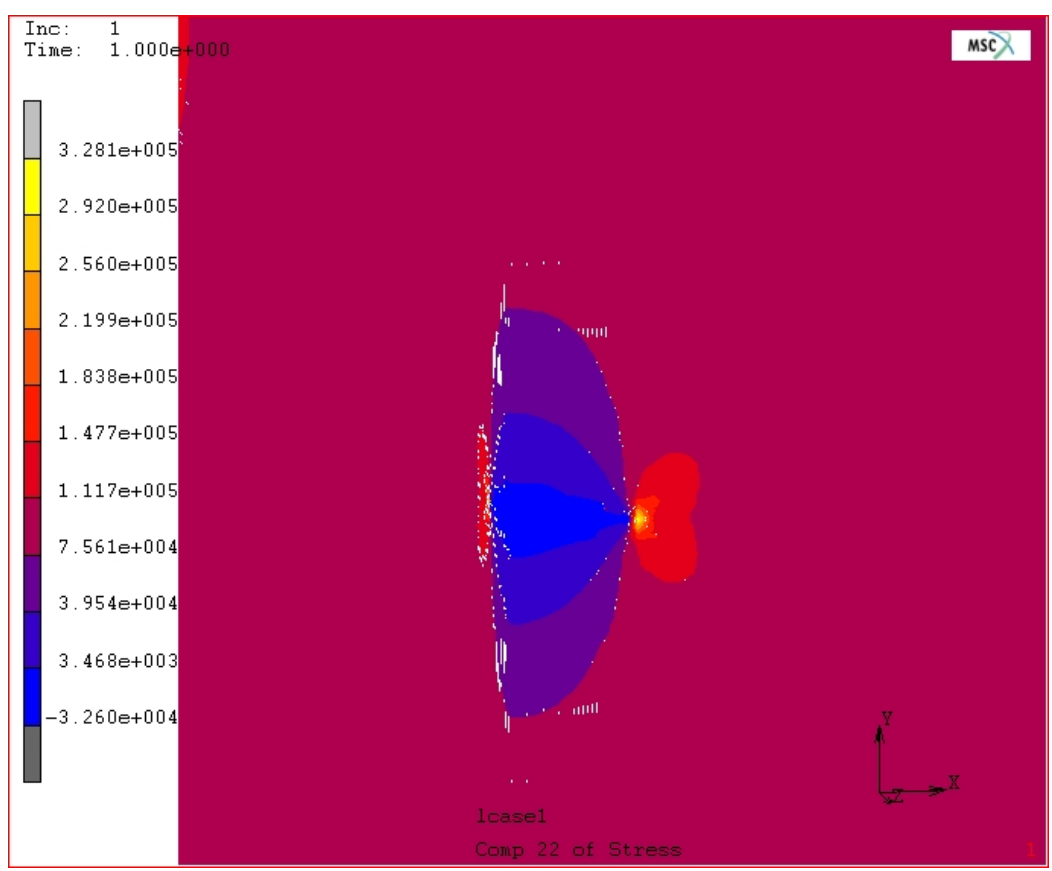

Figure 2-41: Sigma-yy stresses near crack tip

In conclusion the methodology needed to apply the post-processing code FMA to the MARC finite element software has been described and verified for bimaterial cracks at multiple locations in a FEM model. A benefit of the FMA program is its flexibility in use for different finite element codes and also its accuracy. It provides enhanced fracture analysis capabilities for any software.

\subsection{Creep Prediction in Ni/YSZ Cermet Anodes}

\subsubsection{Analytical Creep Model for Ni/YSZ Cermet Anodes}

Our attention is continuously focused on the effects of the particle distribution in the anode. The Ni/YSZ anode is a mixture of $\mathrm{Ni}$ and $\mathrm{YSZ}$, or a cermet. Due to $\mathrm{NiO}$ reduction, the Ni/YSZ anode also contains a large number of voids, making it a porous cermet. In this complicated microstructure, creep deformation will occur predominately in the Ni phase. However, the creep behavior of the anode will be very different from that of bulk $\mathrm{Ni}$, due to the presence of YSZ particles and voids. A constitutive creep law needs to be developed for the anode. In this report, several distributions of Ni and YSZ in the anode are investigated in order to gain better understanding of the creep of $\mathrm{Ni}$ on the creep of mixture of Ni and YSZ. The objective is to understand how creep of $\mathrm{Ni}$ in the anode will affect the creep of the mixture of Ni and YSZ.

To this end, let us consider a Ni/YSZ cermet consists of Ni, YSZ particles and voids. Their volume fractions are, $c_{N}, c_{Y}$ and $c_{V}$, respectively, where we have

$$
c_{N}+c_{Y}+c_{V}=1 .
$$

Since the voids can be viewed as particles with vanishing elastic modulus, the average stress in the cermet and in the Ni and YSZ phases, respectively, can be written as 


$$
\begin{aligned}
& \bar{\sigma}_{i j}=\frac{1}{V} \int_{V} \sigma_{i j} d v=c_{N} \bar{\sigma}_{i j}^{N}+c_{Y} \bar{\sigma}_{i j}^{Y}, \\
& \bar{\sigma}_{i j}^{N}=\frac{1}{V_{N}} \int_{V_{N}} \sigma_{i j} d v, \bar{\sigma}_{i j}^{Y}=\frac{1}{V_{Y}} \int_{V_{Y}} \sigma_{i j} d v .
\end{aligned}
$$

The average strain in the cermet can be written as

$$
\bar{\varepsilon}_{i j}=\frac{1}{V} \int_{V} \varepsilon_{i j} d v=c_{N} \bar{\varepsilon}_{i j}^{N}+c_{Y} \bar{\varepsilon}_{i j}^{Y},
$$

where $V$ is the total volume of the cermet and

$$
\bar{\varepsilon}_{i j}^{N}=\frac{1}{V_{N}} \int_{V_{N}} \varepsilon_{i j}^{N} d v, \quad \bar{\varepsilon}_{i j}^{Y}=\frac{1}{V_{Y}} \int_{V_{Y}} \varepsilon_{i j}^{Y} d v
$$

where the overbar on the strain indicates their averages.

Note that the total strain can be written as the sum of the elastic $\varepsilon_{i j}^{e}$ strain, plastic $\varepsilon_{i j}^{p}$ strain and creep strain $\varepsilon_{k l}^{c}$. Therefore, for each phase (Ni and YSZ), Hooke's law yield

$$
\sigma_{i j}=C_{i j k l} \varepsilon_{k l}^{e}=C_{i j k l}\left(\varepsilon_{k l}-\varepsilon_{k l}^{p}-\varepsilon_{k l}^{c}\right)
$$

where $C_{i j k l}$ is the elasticity tensor. Making use of the Hooke's law, we can rewrite the average stress in each phase as

$$
\begin{aligned}
& \bar{\sigma}_{i j}^{N}=C_{i j k l}^{N}\left(\bar{\varepsilon}_{k l}^{N}-\bar{\varepsilon}_{k l}^{p, N}-\bar{\varepsilon}_{k l}^{c, N}\right) \\
& \bar{\sigma}_{i j}^{Y}=C_{i j k l}^{Y}\left(\bar{\varepsilon}_{k l}^{Y}-\bar{\varepsilon}_{k l}^{p, Y}-\bar{\varepsilon}_{k l}^{c, Y}\right)=C_{i j k l}^{Y} \bar{\varepsilon}_{k l}^{Y}
\end{aligned}
$$

where it was assumed that the plastic and creep strains in the YSZ phase are negligible. The above equations (2.80) and (2.81) may be rewritten as

$$
\begin{aligned}
& \bar{\varepsilon}_{i j}^{N}=S_{i j k l}^{N} \bar{\sigma}_{k l}^{N}+\bar{\varepsilon}_{i j}^{p, N}+\bar{\varepsilon}_{i j}^{c, N} \\
& \bar{\varepsilon}_{i j}^{Y}=S_{i j k l}^{Y} \bar{\sigma}_{k l}^{Y}
\end{aligned}
$$

where

$$
S_{i j k l}^{N}=\left(C_{i j k l}^{N}\right)^{-1}, \quad S_{i j k l}^{Y}=\left(C_{i j k l}^{Y}\right)^{-1}
$$

In the last report, we assume that during the deformation, the total strain in the $\mathrm{Ni}$ and the YSZ phases are the same, i.e.,

$$
\bar{\varepsilon}_{i j}=\frac{1}{V_{Y}} \int_{V_{Y}} \varepsilon_{k l} d v=\frac{1}{V_{N}} \int_{V_{N}} \varepsilon_{k l} d v
$$

Without the consideration of the plasticity of $\mathrm{Ni}$, this leads to 


$$
\bar{\sigma}_{i j}=\left(c_{N} C_{i j k l}^{N}+c_{Y} C_{i j k l}^{Y}\right) \bar{\varepsilon}_{k l}-c_{N} C_{i j k l}^{N} \bar{\varepsilon}_{k l}^{c}=\bar{C}_{i j k l} \bar{\varepsilon}_{k l}-c_{N} C_{i j k l}^{N} \bar{\varepsilon}_{k l}^{c}
$$

where

$$
\bar{C}_{i j k l}=c_{N} C_{i j k l}^{N}+c_{Y} C_{i j k l}^{Y}
$$

is clearly the rule of mixture average of the effective elasticity tensor of the cermet.

Under fixed load, i.e., $\bar{\sigma}_{i j}=$ const., one may obtain the relationship between the effective strain rate and effective stress of the $\mathrm{Ni} / \mathrm{YZS}$ mixture, for isotropic materials, as

$$
\dot{\bar{\varepsilon}}=c_{N} \frac{\mu_{N}}{\bar{\mu}} A \exp \left(-\frac{Q}{R T}\right)\left[\frac{-3 c_{Y} A \mu_{N} \mu_{Y}\left(1-n_{c}\right) t}{2 \bar{\mu}} \exp \left(-\frac{Q}{R T}\right)+\left(\frac{\mu_{N}}{\bar{\mu}} \bar{\sigma}\right)^{1-n_{c}}\right]^{\frac{n_{c}}{1-n_{c}}}
$$

for $n_{c} \neq 1$, and

$$
\dot{\bar{\varepsilon}}=c_{N}\left(\frac{\mu_{N}}{\bar{\mu}}\right)^{2} A \exp \left(-\frac{Q}{R T}\right) \exp \left(\frac{-3 c_{Y} A \exp \left(-\frac{Q}{R T}\right) \mu_{N} \mu_{Y} t}{2 \bar{\mu}}\right) \bar{\sigma}
$$

for $n_{c}=1$. The equations (2.88) and (2.89) give the strain rate (relaxation of) the entire $\mathrm{Ni} / Y S Z$ mixture as a whole as a function of the overall effective stress.

Now, we assume that during the deformation, the stresses in the Ni and the YSZ phases are the same.

$$
\bar{\sigma}_{i j}^{N}=\bar{\sigma}_{i j}^{Y}=\bar{\sigma}_{i j}
$$

Further, we assume that the plastic strain in the Ni phase also can be neglected, which yields

$$
\bar{\varepsilon}_{i j}=\left(c_{N} S_{i j k l}^{N}+c_{Y} S_{i j k l}^{Y}\right) \bar{\sigma}_{k l}+c_{N} \bar{\varepsilon}_{k l}^{c, N}=\bar{S}_{i j k l} \bar{\sigma}_{k l}+c_{N} \bar{\varepsilon}_{k l}^{c, N}
$$

where

$$
\bar{S}_{i j k l}=c_{N} S_{i j k l}^{N}+c_{Y} S_{i j k l}^{Y}
$$

is the rule of mixture average of the effective compliance tensor of the cermet.

Under fixed load, i.e., $\bar{\sigma}_{i j}=$ const., one may take the time derivative of (2.91) to obtain

$$
\dot{\bar{\varepsilon}}_{i j}=C_{N} \dot{\bar{\varepsilon}}_{i j}^{c, N}
$$

It is obvious that the creep of the cermet is completely dominated by the Ni phase in the cermet. The above equation (2.85) may be expressed by Mises effective strain rate as

$$
\dot{\bar{\varepsilon}}=c_{N} \dot{\bar{\varepsilon}}^{c, N}
$$


where the Mises effective and strain are defined by the standard formulas

$$
\begin{aligned}
& \dot{\bar{\varepsilon}}=\sqrt{\frac{2}{3}\left(\dot{\bar{\varepsilon}}_{i j}-\frac{1}{3} \dot{\bar{\varepsilon}}_{k k} \delta_{i j}\right)\left(\dot{\bar{\varepsilon}}_{i j}-\frac{1}{3} \dot{\bar{\varepsilon}}_{k k} \delta_{i j}\right)}, \\
& \dot{\bar{\varepsilon}}^{c}=\sqrt{\frac{2}{3}\left(\dot{\bar{\varepsilon}}_{i j}^{c}-\frac{1}{3} \dot{\bar{\varepsilon}}_{k k}^{c} \delta_{i j}\right)\left(\dot{\bar{\varepsilon}}_{i j}^{c}-\frac{1}{3} \dot{\bar{\varepsilon}}_{k k}^{c} \delta_{i j}\right)} .
\end{aligned}
$$

With the same assumption given in the last report, the Ni phase follows the exponential creep law,

$$
\dot{\bar{\varepsilon}}^{c, N}=B\left(\bar{\sigma}^{N}\right)^{n_{c}}=B \bar{\sigma}^{n_{c}}
$$

with

$$
B=A \exp \left(-\frac{Q}{R T}\right) .
$$

Substitution of (2.90) into (2.86) yields

$$
\dot{\bar{\varepsilon}}=c_{N} A \exp \left(-\frac{Q}{R T}\right) \bar{\sigma}^{n_{c}} .
$$

This equation gives the strain rate (relaxation of) the entire Ni/YSZ mixture as a whole as a function of the overall effective stress.

In deriving the above, it has been assumed that strains in the Ni and YSZ phases uniform, respectively. Consequently, the result shown in (2.88) or (2.89) provides only a lower bound for the rate of relaxation and the result in (2.99) presents an upper bound for rate of relaxation. In an actual Ni/YSZ mixture, the rate of relaxation will be residing between the lower and upper bounds. We may represent the creep of the mixture with the combination of the equations obtained above as

$$
\dot{\bar{\varepsilon}}=c_{N} A \exp \left(-\frac{Q}{R T}\right)\left(\alpha_{1} \frac{\mu_{N}}{\bar{\mu}}\left[\frac{-3 c_{Y} A \mu_{N} \mu_{Y}\left(1-n_{c}\right) t}{2 \bar{\mu}} \exp \left(-\frac{Q}{R T}\right)+\left(\frac{\mu_{N}}{\bar{\mu}} \bar{\sigma}\right)^{1-n_{c}}\right]^{\frac{n_{c}}{1-n_{c}}}+\alpha_{2} \bar{\sigma}^{n_{c}}\right)
$$

for $n_{c} \neq 1$, and

$$
\dot{\bar{\varepsilon}}=c_{N} A \exp \left(-\frac{Q}{R T}\right)\left[\alpha_{1}\left(\frac{\mu_{N}}{\bar{\mu}}\right)^{2} \exp \left(\frac{-3 c_{Y} A \exp \left(-\frac{Q}{R T}\right) \mu_{N} \mu_{Y} t}{2 \bar{\mu}}\right)+\alpha_{2}\right] \bar{\sigma}
$$

for $n_{c}=1$. Here $\alpha_{1}$ and $\alpha_{2}$ are the influence parameters of the two distribution of the particles for the creep of the mixture of Ni and YSZ.

To obtain the influence parameters mentioned above, we consider the mixture of $\mathrm{Ni}$ and YSZ as Ni matrix with the spherical inclusions of YSZ in the following section. 
The linear visco-elastic problem may be transferred into the elastic problem in the transformed domain by means of Laplace transformation. The visco-elastic solution can be obtained by the Laplace inversion of the elastic solution obtained in the transformed domain.

Here, the creep of $\mathrm{Ni}$ is characterized by Maxwell model as

$$
\dot{\varepsilon}^{N}=\frac{1}{3 \eta_{N}} \sigma^{N}
$$

Comparison of (1.29) with (1.24) when $n_{c}=1$ yields

$$
\frac{1}{3 \eta_{N}}=A e^{-Q / R T}
$$

The relationships of the hydrostatic and deviator stress strain are expressed as

$$
\begin{aligned}
& \varepsilon_{k k}=\frac{1}{3 \kappa} \sigma_{k k}, \\
& \varepsilon_{i j}-\frac{1}{3} \varepsilon_{k k}=\frac{1}{2 \mu}\left(\sigma_{i j}-\frac{1}{3} \sigma_{k k}\right)
\end{aligned}
$$

For the elastic problem of Ni matrix with YSZ spherical inclusions, the overall bulk and shear modulus may be written as

$$
\begin{gathered}
\kappa=\kappa_{N}\left(1+\frac{a}{1-\gamma_{1} a}\right), \\
\mu=\mu_{N}\left(1+\frac{b}{1-\gamma_{2} b}\right),
\end{gathered}
$$

where

$$
\begin{aligned}
& \gamma_{1}=\frac{3 \kappa_{N}}{3 \kappa_{N}+4 \mu_{N}}, \\
& \gamma_{2}=\frac{6}{5} \frac{\kappa_{N}+2 \mu_{N}}{3 \kappa_{N}+4 \mu_{N}}, \\
& a=\frac{c_{Y}\left(\kappa_{Y}-\kappa_{N}\right)}{\gamma_{1}\left(\kappa_{Y}-\kappa_{N}\right)+\kappa_{N}}, \\
& b=\frac{c_{Y}\left(\mu_{Y}-\mu_{N}\right)}{\gamma_{2}\left(\mu_{Y}-\mu_{N}\right)+\mu_{N}} .
\end{aligned}
$$

Substation of (2.106) and (2.107) into (2.102) and (2.103), ones may get expression as

$$
\begin{aligned}
& \kappa=\kappa_{N}\left(1+\frac{c_{Y}\left(\kappa_{Y}-\kappa_{N}\right)}{c_{N} \gamma_{1}\left(\kappa_{Y}-\kappa_{N}\right)+\kappa_{N}}\right) \\
& \mu=\mu_{N}\left(1+\frac{c_{Y}\left(\mu_{Y}-\mu_{N}\right)}{c_{N} \gamma_{2}\left(\mu_{Y}-\mu_{N}\right)+\mu_{N}}\right)
\end{aligned}
$$

By means of the correspondence principle the effective stress-strain relations of an isotropic viscoelastic composite in the transformed domain can be written in terms of its effective transformed bulk and shear moduli as 


$$
\begin{aligned}
& \hat{\varepsilon}_{k k}=\frac{1}{3 \kappa^{T D}} \hat{\sigma}_{k k}, \\
& \hat{\varepsilon}_{i j}-\frac{1}{3} \hat{\varepsilon}_{k k}=\frac{1}{2 \mu^{T D}}\left(\hat{\sigma}_{i j}-\frac{1}{3} \hat{\sigma}_{k k}\right)
\end{aligned}
$$

where

$$
\begin{aligned}
& \kappa^{T D}=\kappa_{N}^{T D}\left(1+\frac{c_{Y}\left(\kappa_{Y}^{T D}-\kappa_{N}^{T D}\right)}{c_{N} \gamma_{1}^{T D}\left(\kappa_{Y}^{T D}-\kappa_{N}^{T D}\right)+\kappa_{N}^{T D}}\right) \\
& \mu^{T D}=\mu_{N}^{T D}\left(1+\frac{c_{Y}\left(\mu_{Y}^{T D}-\mu_{N}^{T D}\right)}{c_{N} \gamma_{2}^{T D}\left(\mu_{Y}^{T D}-\mu_{N}^{T D}\right)+\mu_{N}^{T D}}\right)
\end{aligned}
$$

with

$$
\begin{aligned}
& \gamma_{1}^{T D}=\frac{3 \kappa_{N}^{T D}}{3 \kappa_{N}^{T D}+4 \mu_{N}^{T D}}, \\
& \gamma_{2}^{T D}=\frac{6}{5} \frac{\kappa_{N}^{T D}+2 \mu_{N}^{T D}}{3 \kappa_{N}^{T D}+4 \mu_{N}^{T D}},
\end{aligned}
$$

The subscript (.) ${ }^{T D}$ refers to transformed domain.

Equation (2.115) can be rewritten in term of Mises effective form as

$$
\hat{\bar{\varepsilon}}=\frac{1}{3 \mu^{T D}} \hat{\bar{\sigma}}
$$

After Laplace inversion of (2.120)(1.47), ones get

$$
\dot{\bar{\varepsilon}}=\frac{1}{3 \eta_{\mu}} \bar{\sigma}
$$

where

$$
\frac{1}{\eta_{\mu}(t)}=\frac{T_{0}}{\mu}\left[\frac{d_{6}}{d_{8}}+\frac{\left(s_{3}-s_{1}\right)\left(s_{3}-s_{2}\right)}{s_{3}\left(s_{3}-s_{4}\right)}\left(1-s_{3}\right) e^{-s_{3} T_{0} t}+\frac{\left(s_{4}-s_{1}\right)\left(s_{4}-s_{2}\right)}{s_{4}\left(s_{4}-s_{3}\right)}\left(1-s_{4}\right) e^{-s_{4} T_{0} t}\right]
$$

with

$$
\begin{aligned}
& d_{1}=\frac{c_{Y}\left(\kappa_{Y}-\kappa_{N}\right)}{c_{N} \gamma_{1}\left(\kappa_{Y}-\kappa_{N}\right)+\kappa_{N}} \\
& d_{2}=\frac{c_{N} \kappa_{Y}+c_{Y} \kappa_{N}}{c_{N} \gamma_{1}\left(\kappa_{Y}-\kappa_{N}\right)+\kappa_{N}} \gamma_{1} \\
& d_{3}=\frac{d_{2}+d_{1} \gamma_{1}}{1+d_{1}} \\
& d_{4}=\frac{\mu_{Y}-\mu_{N}}{c_{N} \gamma_{2}\left(\mu_{Y}-\mu_{N}\right)+\mu_{N}} \\
& d_{5}=\left[c_{N} \gamma_{2}\left(\frac{\kappa_{N}}{\kappa_{N}+2 \mu_{N}}+\frac{\mu_{Y}}{\mu_{Y}-\mu_{N}}\right)+\frac{\mu_{N}}{\mu_{Y}-\mu_{N}} \gamma_{1}\right] d_{4}
\end{aligned}
$$




$$
\begin{aligned}
& d_{7}=\frac{\mu_{N}}{\mu}\left[d_{5}+c_{Y} d_{4}\left(\frac{\mu_{Y}}{\mu_{Y}-\mu_{N}}+\gamma_{1}\right)\right] \\
& d_{8}=\frac{\mu_{N}}{\mu}\left[d_{6}+c_{Y} d_{4}\left(\frac{\mu_{Y}}{\mu_{Y}-\mu_{N}}+\gamma_{1}\right)\right] \\
& s_{1,2}=\frac{1}{2}\left[d_{5} \pm\left(d_{5}^{2}-4 d_{6}\right)^{1 / 2}\right] \\
& s_{3,4}=\frac{1}{2}\left[d_{7} \pm\left(d_{7}^{2}-4 d_{8}\right)^{1 / 2}\right] \\
& T_{0}=\frac{\mu_{N}}{\eta_{N}}
\end{aligned}
$$

Parameters $\alpha_{1}$ and $\alpha_{2}$ may be determined by means of the comparison of (2.121)( and (2.102) as

$$
\begin{aligned}
& \alpha_{1}=\frac{\eta_{N}}{c_{N}} \frac{T_{0}}{\mu}\left(\frac{\bar{\mu}}{\mu_{N}}\right)^{2} \frac{h_{1} e^{-s_{3} T_{0} t}+h_{2} e^{-s_{4} T_{0} t}}{e^{-c_{Y} \mu_{N} \mu_{Y} T_{0} t / 2 \bar{\mu} \eta_{N}}}, \\
& \alpha_{2}=\frac{\eta_{N}}{c_{N}} \frac{T_{0}}{\mu} \frac{d_{6}}{d_{8}}
\end{aligned}
$$

with

$$
\begin{aligned}
& h_{1}=\frac{\left(s_{3}-s_{1}\right)\left(s_{3}-s_{2}\right)}{s_{3}\left(s_{3}-s_{4}\right)}\left(1-s_{3}\right), \\
& h_{2}=\frac{\left(s_{4}-s_{1}\right)\left(s_{4}-s_{2}\right)}{s_{4}\left(s_{4}-s_{3}\right)}\left(1-s_{4}\right) .
\end{aligned}
$$

With the parameters $\alpha_{1}$ and $\alpha_{2}$ obtained above, we can determine the creep of the mixture by means of equation (2.101) or (2.102) for $n_{c} \neq 1$ or $n_{c}=1$, respectively.

\subsection{2. $\quad$ FEM creep models for reduced $\mathrm{Ni} / \mathrm{YSZ}$ anodes}

Previously, an analytical model was developed for the creep deformation of $\mathrm{Ni} / Y S Z$ cermet. To verify the accuracy of the analytical model, the finite element method is used to numerically investigate the creep behavior of the $\mathrm{Ni} / \mathrm{YSZ}$ cermet.

To this end, the actual microstructure of a Ni/YSZ is considered here. Figure 2-42 shows an SEM micrograph of a Ni/YSZ cermet. Figure 2-42a gives the Ni phase, and Figure 2-42b gives the YSZ phase. The contents of Ni and YSZ are $39 \%$ and $31 \%$, respectively. The rest is void, taking $30 \%$ of the total volume. Based on the gray scale of the SEM micrograph, a solid model is created for the Ni and YSZ phases, respectively. Figure 2-43 shows the superposition of Figure 2-42 a and b. Figure 2-45 shows the mesh used in FEA with 32400 elements. Figure 2-46 show the curves of the creep strain over time under constant loading. It is shown that in the real structure, creep of Ni/YSZ obtained by FEM is larger than that predicted by our analytical model. In Figure 2-47, the curve of creep model is obtained by analytical expression with $\beta=0.44$. It shows that the curves obtained by creep model and FEM take good agreement. 


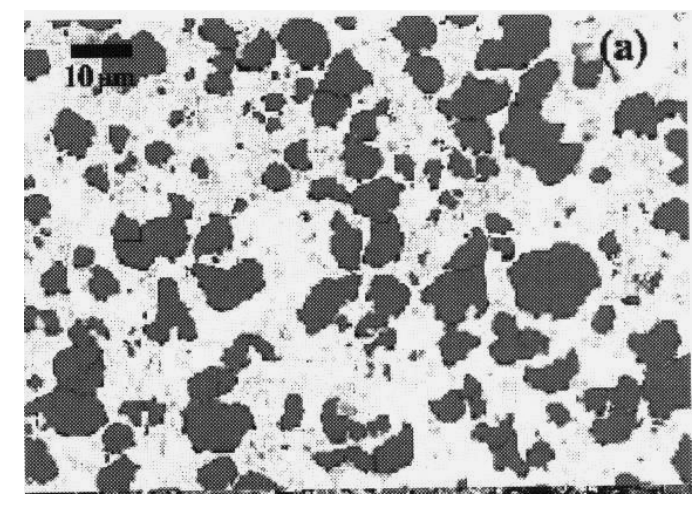

(a) Ni phase

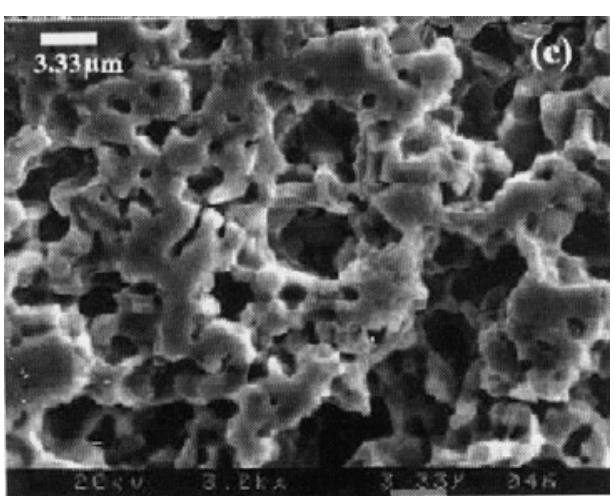

(b) YSZ phase

Figure 2-42: Distribution of Ni and YSZ

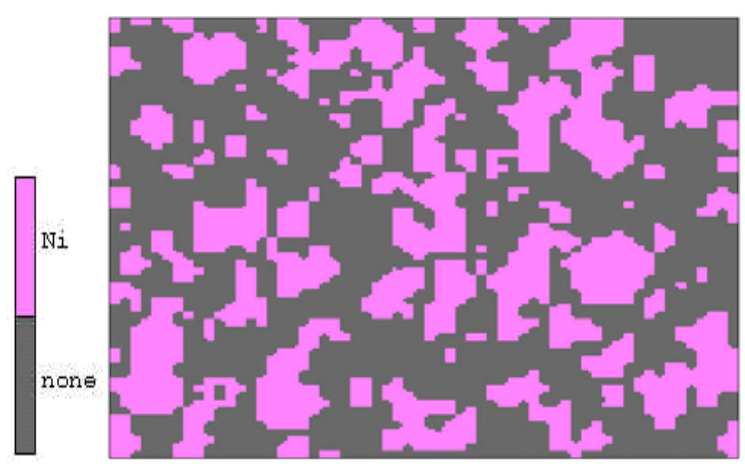

(a) Ni phase

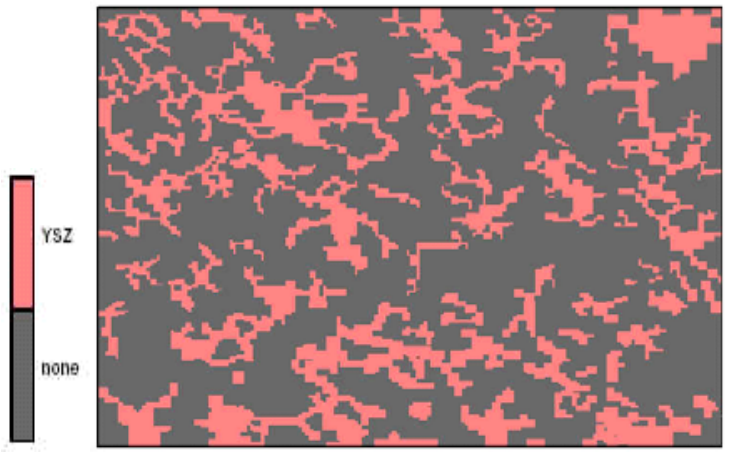

(b) YSZ phase

Figure 2-43: Approximation of Ni and YSZ particles used in FEA 


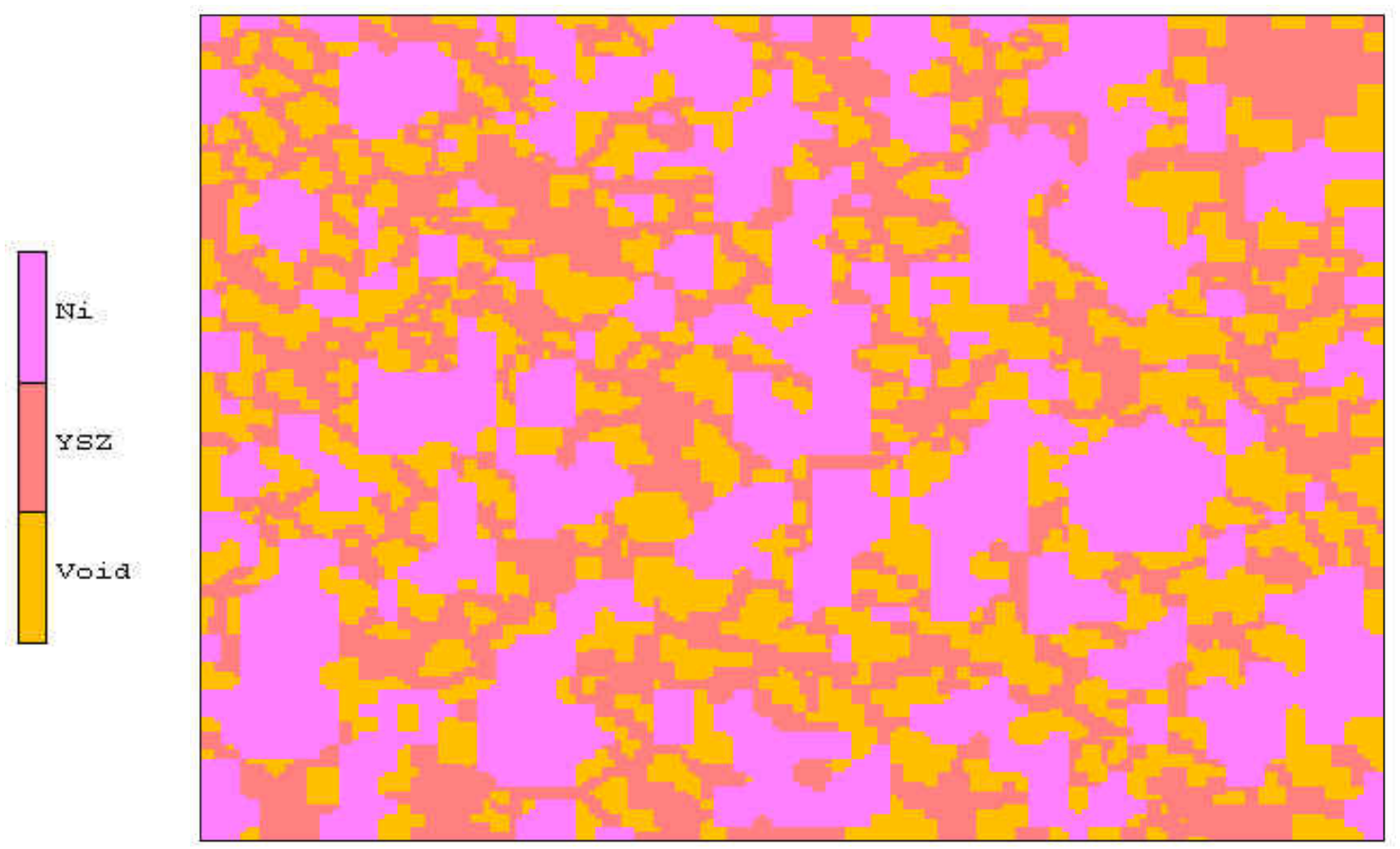

Figure 2-44: Overall distribution of Ni, YSZ and void

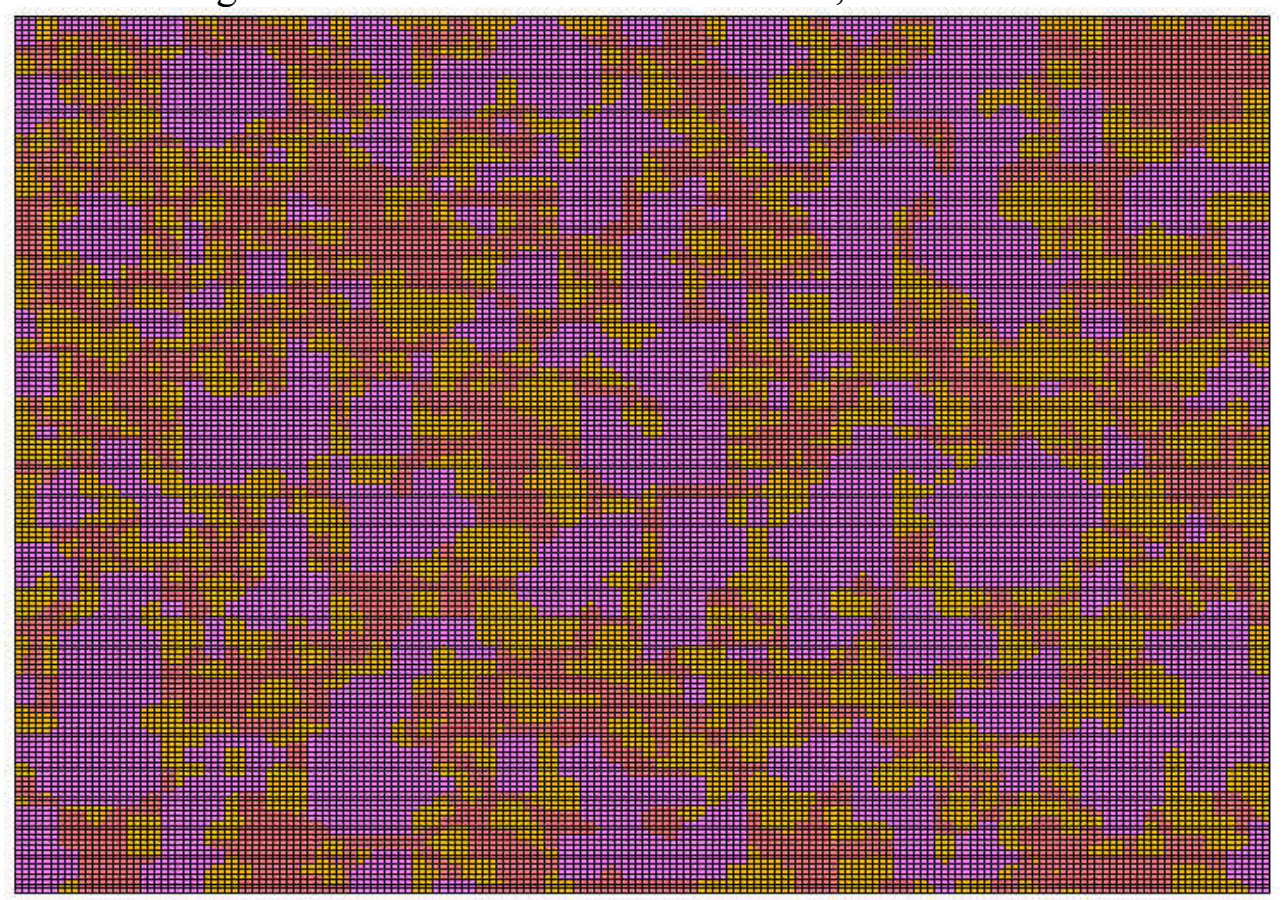

Figure 2-45: Mesh used in FEA 


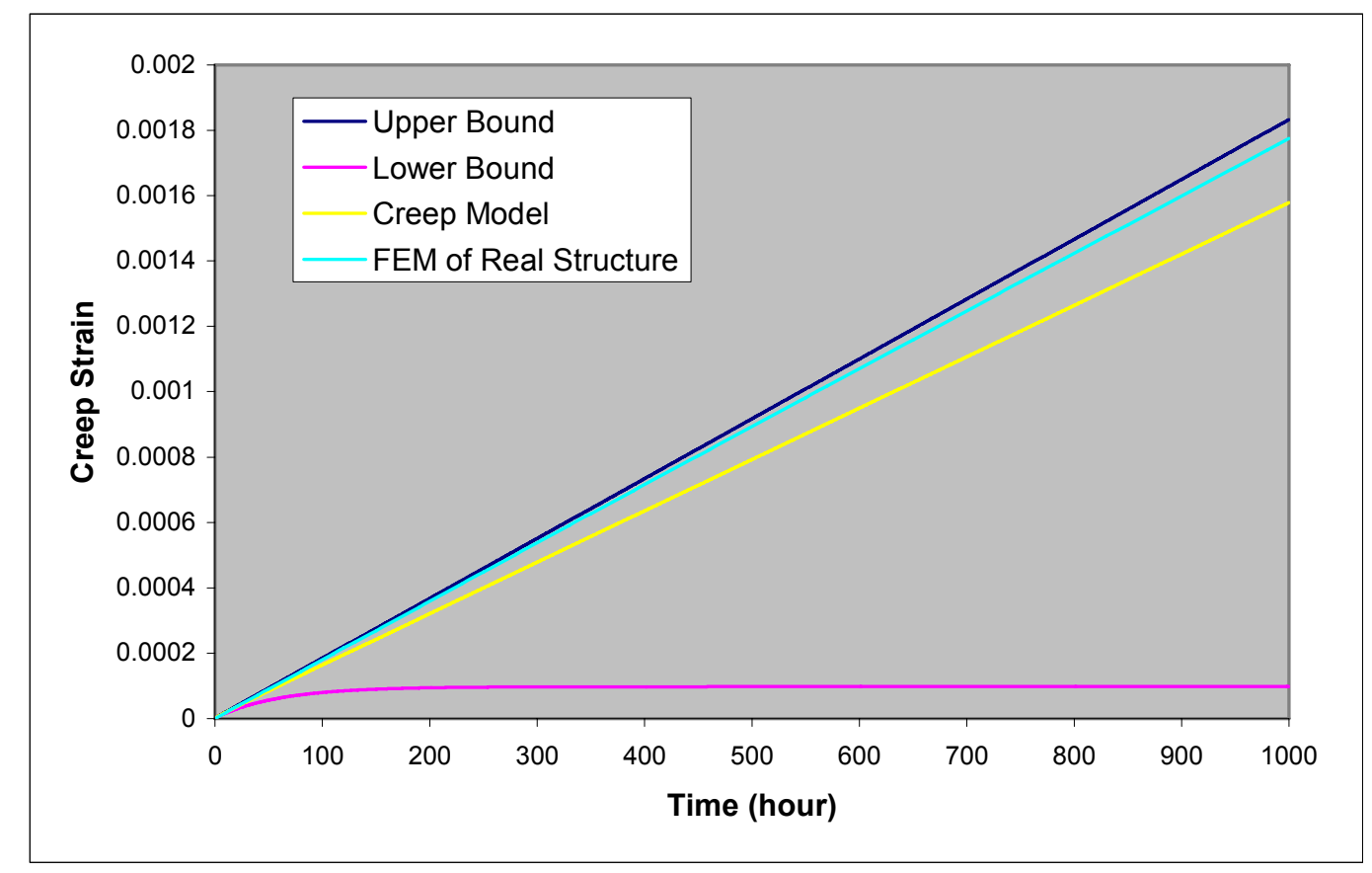

Figure 2-46: Curves of creep over time

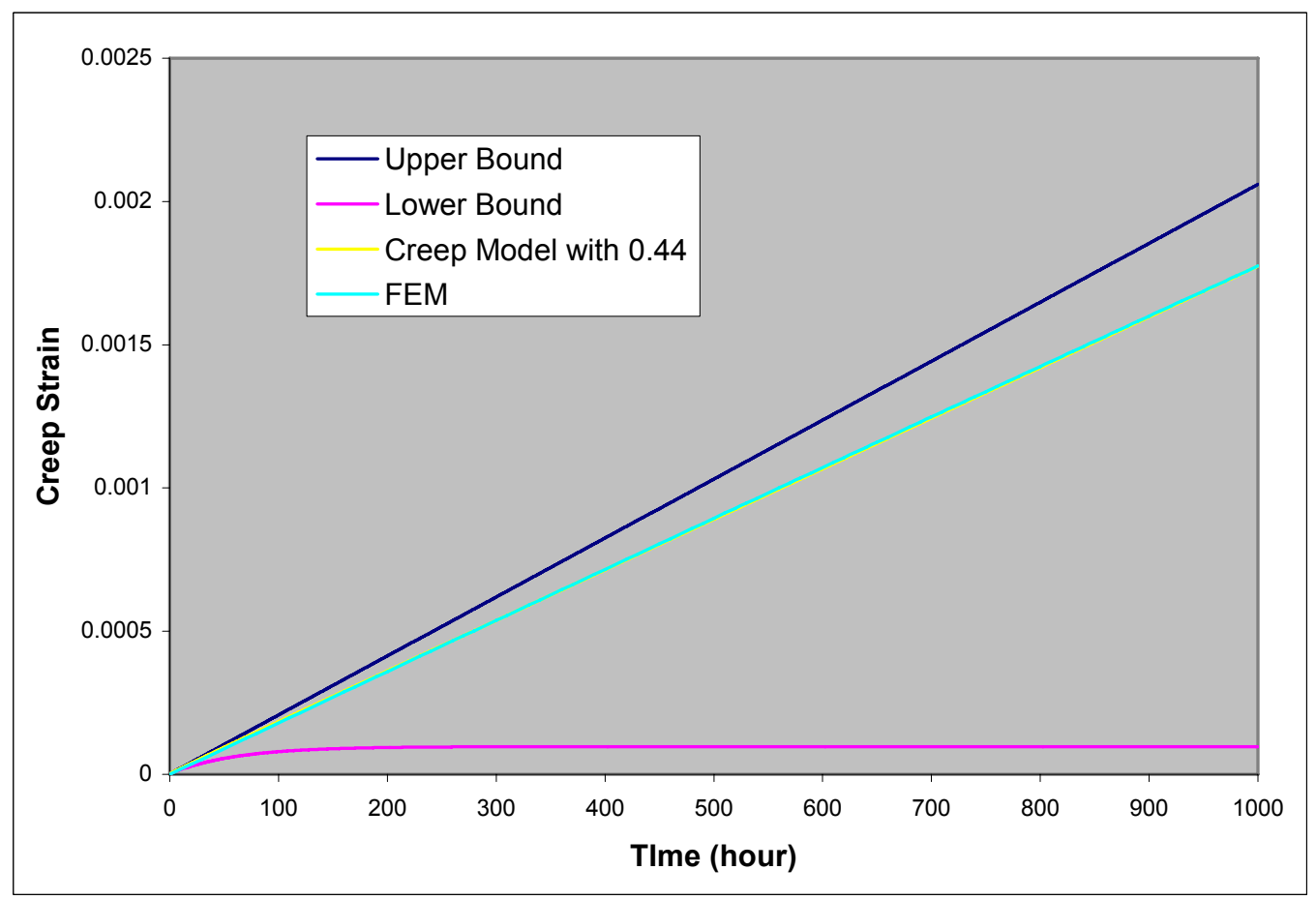

Figure 2-47: Curves of creep over time by modified creep model with $\beta=0.44$

\subsection{First Order Failure Criteria for SOFC PEN Structure}

This section summarizes the first order failure criteria to be used for the initial design against mechanical failure of the PEN structure in high temperature SOFCs. The meaning of terms "first order" used here is twofold. First, we consider only the initial failures occurred during either cell/stack assembly, or the first few cycles of operation. 
Second, we consider only the simplistic failure theories, i.e., theories neglecting the higher order effects such as thermal aging, rate dependency, multi-physics coupling, etc. The purposes of developing such first order failure criteria are to aid the initial design, material selection and optimization of SOFCs.

The first order failure criteria are classified into two groups, namely, local and global. The local failure criteria are formulated based on the knowledge of local stress fields. It is assumed here that the users have conducted stress analysis and obtained stress distribution in the PEN structure. Then, using the local failure criteria, the user can predict (estimate) the potential material failure. Presumably, such exercises are performed computationally so that if failure is predicted based on the first order failure criteria, changes can be made in the design to lower the stresses until the desired factor of safety is reached. The global failure criteria are formulated based on the warpage of the tri-layer PEN structure (a cell) after processing. Here, it is assumed that the warpage of each cell is measured before stack assembly. Then, using the global failure criteria, the user can predict whether a cell can survive the stacking assembly process. Clearly, such global failure criteria can also be used for designing the sintering processes in order to void excess warpage of the cells.

\subsubsection{Local Failure Criteria}

It is assumed that the stress fields in the PEN structure have been obtained (either numerical, or analytically, or experimentally). Thus, the corresponding principle stresses can be computed. The principal normal stresses will be denoted by $\sigma_{1} \geq \sigma_{2} \geq \sigma_{3}$, while the principal shear stresses will be denoted by

$$
\tau_{1}=\frac{\left|\sigma_{2}-\sigma_{3}\right|}{2}, \tau_{2}=\frac{\left|\sigma_{1}-\sigma_{3}\right|}{2}, \tau_{3}=\frac{\left|\sigma_{2}-\sigma_{1}\right|}{2}
$$

The effective stress can be written formally as

$$
\bar{\sigma}=f\left(\sigma_{1}, \sigma_{2}, \sigma_{3}\right),
$$

where $f$ is a function to be discussed below. The failure criteria can then be stated formally as follows. Failure occurs when

$$
\left.\bar{\sigma}=\sigma_{f} \quad \text { (at failure }\right),
$$

where $\sigma_{f}$ is the "strength" of the material to be discussed below. Failure is not expected if $\bar{\sigma}$ is less than $\sigma_{f}$, i.e.,

$$
\bar{\sigma}<\sigma_{f} \text { (no failure). }
$$

Also, the factor of safety against failure is given by

$$
X=\frac{\sigma_{f}}{\bar{\sigma}} .
$$

In what follows, we will summarize the functional forms for the function $f$ and the value to be used for $\sigma_{f}$. 
The above failure criterion is applicable when the material does not contain initial flaws. When initial crack-like flaws of appreciable size exist in the material, a fracture criterion should be used. It is assumed here that the stress field near the crack tip is obtained (either numerically, or analytically or experimentally). Thus, the stress intensity factors $K_{I}, K_{I I}$ and $K_{I I I}$ can be computed ${ }^{1}$. The stress intensity factors here play similar role as do the principle normal stresses in failure analysis. The corresponding energy release rate is given by

$$
G=\frac{1-v^{2}}{E}\left(K_{I}^{2}+K_{I I}^{2}+\frac{K_{I I I}^{2}}{1-v}\right)
$$

The fracture criterion can then be formally stated as follows. Fracture occurs when

$$
G=G_{c} \quad(\text { at fracture })
$$

where $G_{c}$ is the fracture toughness of the material to be discussed below. Fracture is not expected when $\mathrm{G}$ is less than $G_{c}$, i.e.

$$
G<G_{c} \text { (no fracture). }
$$

Similar, the factor of safety against fracture is defined as

$$
Y=\frac{G_{c}}{G}
$$

\subsection{2. $\underline{\text { YSZ Electrolyte }}$}

At temperature below $850^{\circ} \mathrm{C}$, YSZ can be considered as a brittle material and its thermomechanical behavior can be accurately represented by the linear elastic constitutive law (Hooke's law).

\section{Maximum Normal Stress Criterion}

Failure occurs when $\bar{\sigma}=\sigma_{f}$, where

$$
\begin{aligned}
& \bar{\sigma}=f\left(\sigma_{1}, \sigma_{2}, \sigma_{3}\right)=\max \left\{\left|\sigma_{1}\right|,\left|\sigma_{2}\right|,\left|\sigma_{3}\right|\right\} \\
& \sigma_{f}=\text { uniaxial tensile strength }=100 \sim 300 \mathrm{MPa}^{2}
\end{aligned}
$$

\section{Fracture Criterion}

Fracture occurs when $G=G_{c}$, where

$$
\begin{aligned}
& G=\frac{1-v^{2}}{E}\left(K_{I}^{2}+K_{I I}^{2}+\frac{K_{I I I}^{2}}{1-v}\right) \\
& G_{c}=7.8 \sim 13.7 \mathrm{~J} / \mathrm{m}^{2}
\end{aligned}
$$

\footnotetext{
${ }^{1}$ Computation of the stress intensity factor using the finite element method has been discussed in our previously reports.

${ }^{2}$ See SECA Material Database by Edgar Lara-Curzio of ORNL.
} 
These values are converted from the fracture toughness data in SECA Material Database (Edgar Lara-Curzio).

\subsubsection{YSZ/Ni Anode}

The YSZ/Ni cermet is a mixture of YSZ and Ni. Typical anodes consist of $40 \%$ volume fraction of Ni, 30\% YSZ and 30\% voids. Microscopic analysis shows that the YSZ phase forms a continuous skeleton of the cermet. Therefore, the YSZ/Ni cermet can be considered as a composite material with $\mathrm{Ni}$ being the matrix reinforced by a continuous YSZ phase. Because of the high volume fractions of the void and YSZ phases, it is reasonable to assume that the strength of the cermet is approximately the average stress in the cermet at the YSZ failure strain. Thus, the failure criterion can be stated as the following:

\section{Maximum Normal Stress Criterion}

Failure occurs when $\bar{\sigma}=\sigma_{f}$, where

$$
\begin{aligned}
& \bar{\sigma}=f\left(\sigma_{1}, \sigma_{2}, \sigma_{3}\right)=\max \left\{\left|\sigma_{1}\right|,\left|\sigma_{2}\right|,\left|\sigma_{3}\right|\right\} \\
& \sigma_{f}=\sigma_{Y S Z}\left[V_{Y S Z}+\frac{E_{N i}}{E_{Y S Z}\left(1-v_{N i}\right)}\left(1-V_{Y S Z}-V_{\text {Void }}\right)\right]
\end{aligned}
$$

and

$$
\begin{aligned}
& \sigma_{Y S Z}=100 \sim 300 \mathrm{MPa}=\text { YSZ tensile strength } \\
& E_{N i}=\text { Ni Young's modulus } \\
& E_{Y S Z}=\text { YSZ Young's modulus } \\
& v_{N i}=\text { Ni Poisson's ratio } \\
& V_{Y S Z}=\text { YSZ Volume fraction } \\
& V_{\text {Void }}=\text { Void Volume fraction }
\end{aligned}
$$

\subsubsection{Global Failure Criteria}

In this report, the global failure criteria are established based on local fracture failure, because such failure tends to be catastrophic. The types of facture considered here are schematically illustrated in Figure 2-48: Various cracks in a cell below. 


\section{A}

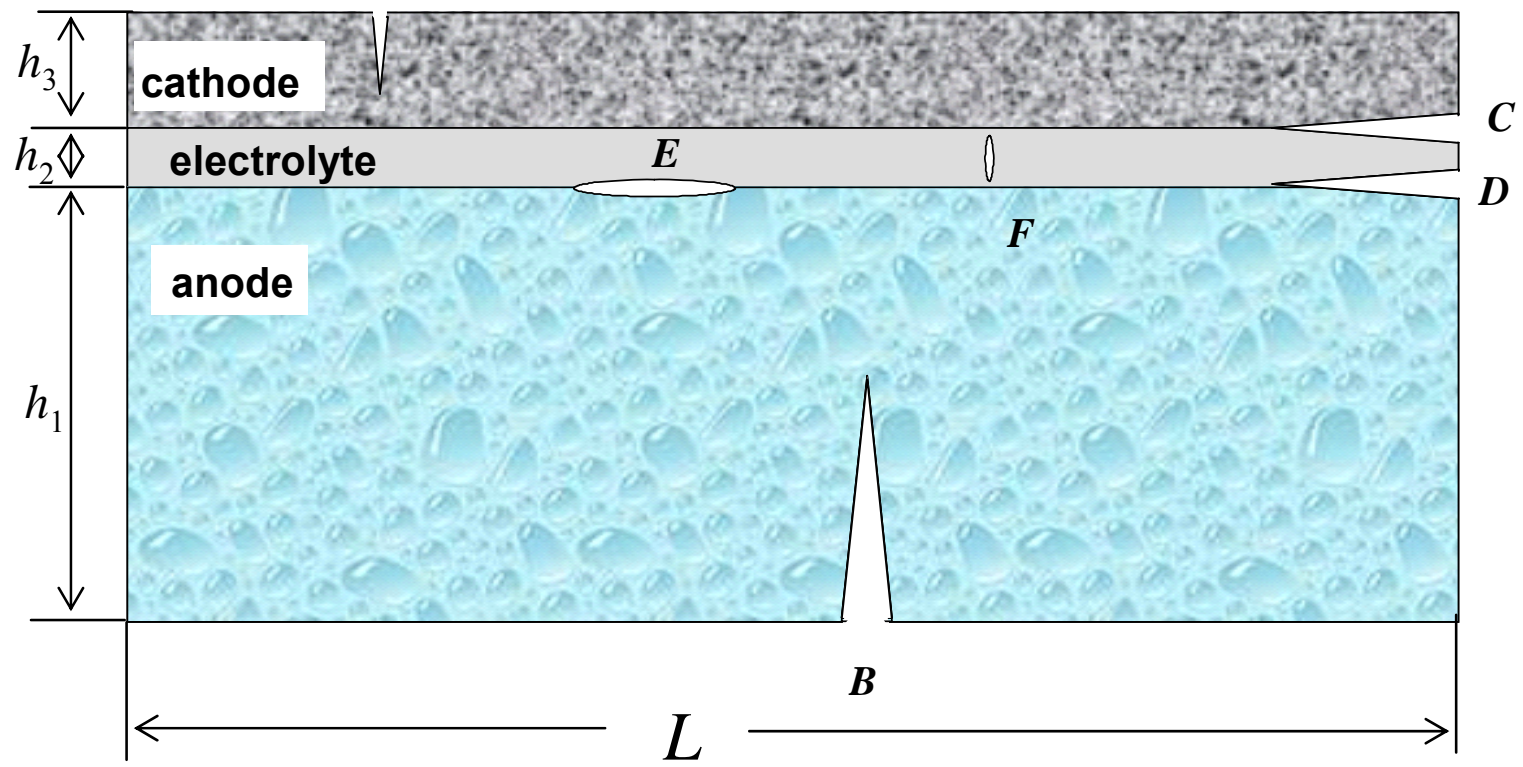

Figure 2-48: Various cracks in a cell
A - crack in the cathode
$\mathrm{B}$ - crack in the anode
$\mathrm{C}$ - delamination crack between the cathode and electrolyte
$\mathrm{D}$ - delamination crack between the anode and the electrolyte
$\mathrm{E}$ - blister crack on the anode/electrolyte interface
$\mathrm{F}$ - crack in the electrolyte

Although various techniques have been developed for sintering the cells, certain warpage of the cell remains after sintering. During the stacking process, cells are flattened in order to be fitted into the cell stack. Flattening exerts additional stresses to the warped cells. Such stress may fracture the cell during the flattening process, if the warpage is excess. For most anode-supported, YSZ based SOFCs, the crack types $A, B$, $C$ and $D$ are particularly vulnerable to the flattening process. The failure criterion below provides the first order estimate of the maximum allowable warpage without fracture and maximum allowable curvature during the stack assembly process,

$$
\begin{aligned}
\frac{W_{c}}{L} & =Y \sqrt{\frac{G_{c}}{h_{2} E_{2}}}\left(\frac{L}{h_{2}}\right) \\
\rho_{c} & =\bar{Y} \sqrt{\frac{G_{c}}{h_{2} E_{2}}}
\end{aligned}
$$

where $W$ is the maximum allowable warpage as illustrated in Figure 2-49, $L$ is the cell size, $E_{2}$ is the Young's modulus of the electrolyte, $h_{2}$ is the thickness of the electrolyte. In this equation, $G_{c}$ is the fracture toughness of the material in which the crack is located and $Y$ is a dimensionless constant that depends on the cell geometry, material constants, 
and crack size and location. The expressions of $Y$ for the cracks showing in Figure 2-48 are given in the Appendix C.
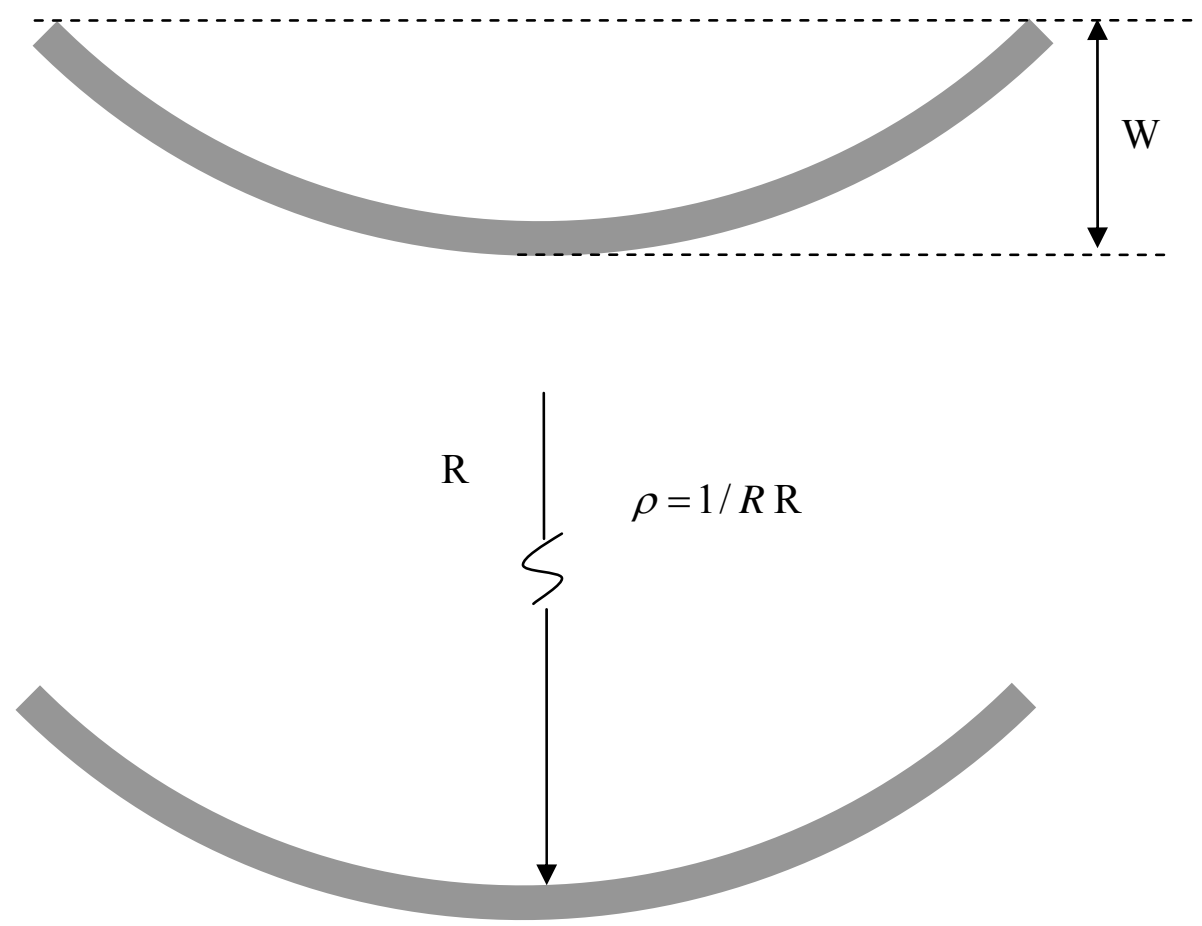

Figure 2-49: A cell with warpage $W$

As an example, consider a cell with geometry and material parameters listed in Table 2-13. For this example, the normal stresses are tensile in the cathode, and compressive in the electrolyte and anode. Therefore, cracks types $A, C$ and $D$ may propagate as the cell is flattened. The values of $Y$ corresponding to these cracks are listed in Table 2-14. For $L=10 \mathrm{~cm}$, the maximum allowable warpage $W$ is plotted in Figure 2-50 against the fracture toughness $G_{c}$ for these cracks. Figure 2-51 includes the maximum allowable curvature.

Table 2-13: Material properties and geometric parameters of a cell

\begin{tabular}{|c|c|c|c|c|}
\hline & $\begin{array}{c}\text { Young's Modulus } \\
(\mathrm{GPa})\end{array}$ & $\begin{array}{c}\text { Poisson's } \\
\text { ratio }\end{array}$ & $\mathrm{CTE}\left(10^{-6} /{ }^{\circ} \mathrm{C}\right)$ & $\begin{array}{c}\text { Thickness } \\
(\mu \mathrm{m})\end{array}$ \\
\hline Cathode & 90 & 0.3 & 11.7 & 75 \\
\hline Electrolyte & 200 & 0.3 & 10.8 & 15 \\
\hline Anode & 96 & 0.3 & 11.2 & 500 \\
\hline
\end{tabular}


Table 2-14: Values of the factor $Y$

\begin{tabular}{|c|c|c|c|c|c|c|}
\hline & \multicolumn{4}{|c|}{ Crack $A$} & \multirow{2}{*}{ Crack $C$} & \multirow{2}{*}{ Crack $D$} \\
\cline { 2 - 5 } & $a=0.01 h_{3}$ & $a=0.05 h_{3}$ & $a=0.1 h_{3}$ & $a=0.2 h_{3}$ & & \\
\hline $\mathrm{Y}$ & $4.63 \mathrm{e}-3$ & $2.08 \mathrm{e}-3$ & $1.48 \mathrm{e}-3$ & $1.06 \mathrm{e}-3$ & $3.87 \mathrm{e}-3$ & $2.01 \mathrm{e}-3$ \\
\hline
\end{tabular}

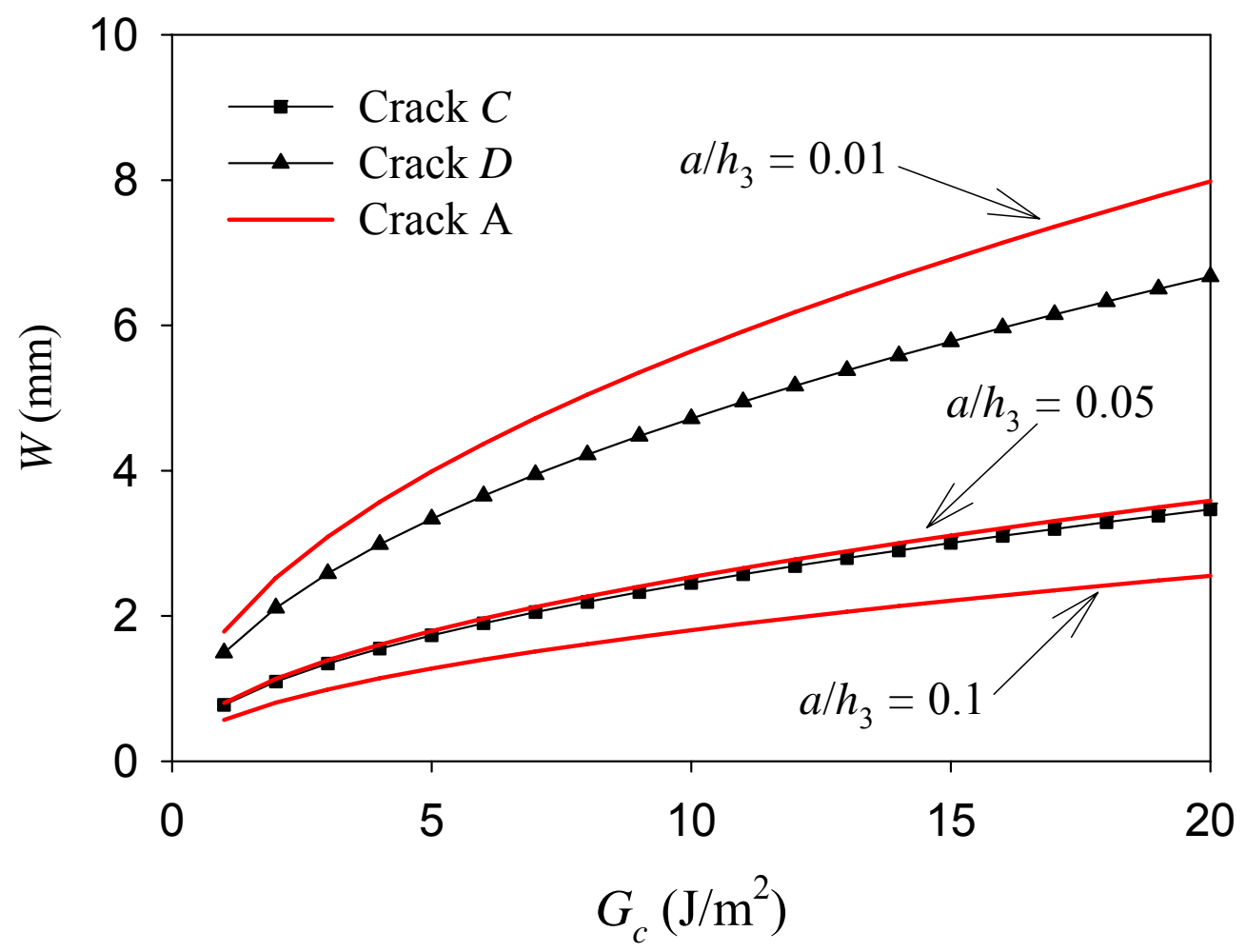

Figure 2-50: W vs. $G_{c}$ for the cell parameters given in Table 2-13 


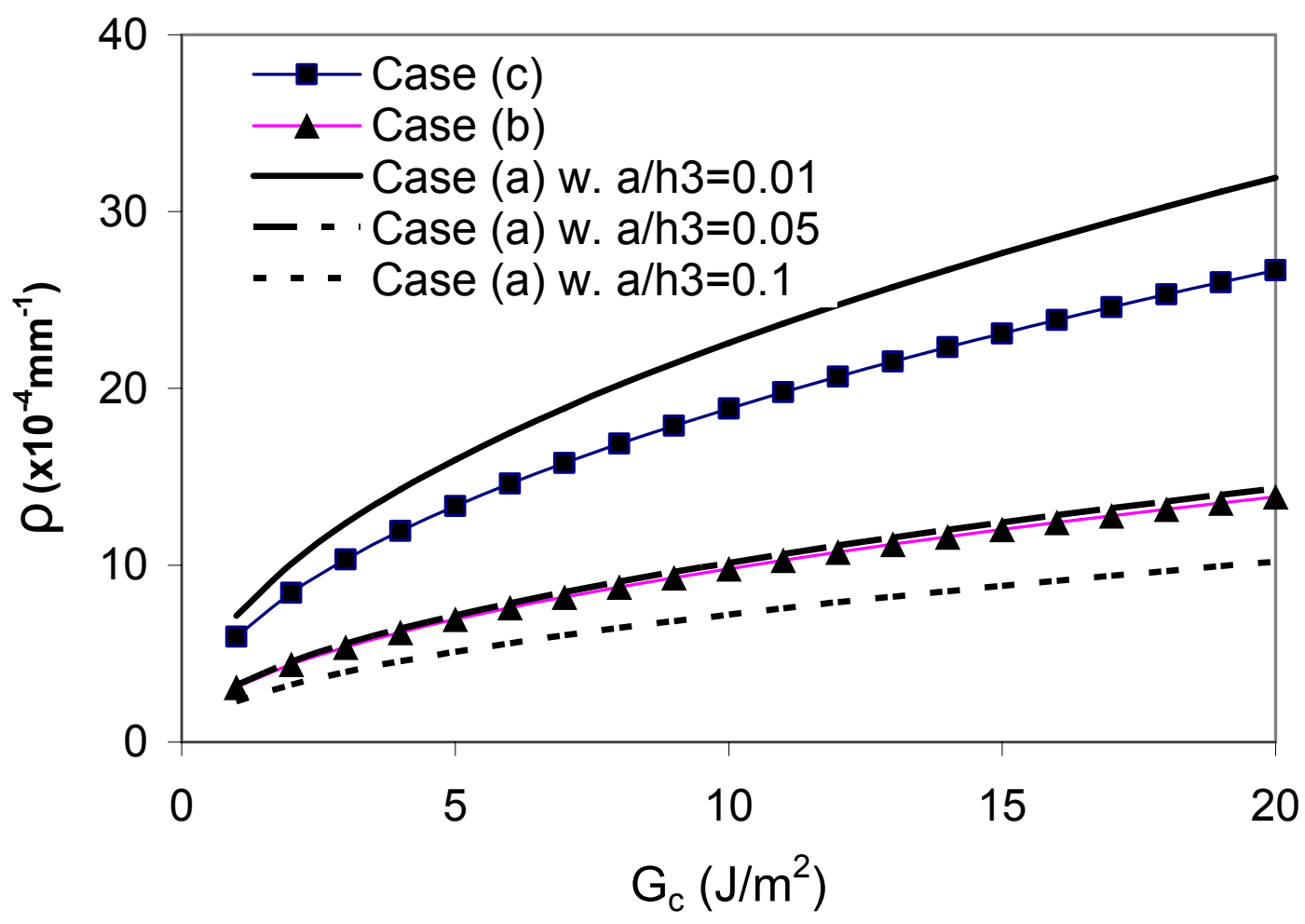

Figure 2-51 $\rho$ vs. $G_{c}$ for the cell parameters given in Table 2-13

In turn, it should be noted that the stress situation in the electrode and electrolyte is closely dependent on the combination of materials in the cell. If CTE of the electrolyte is larger than the electrodes, the temperature drop from the sintering to room atmosphere will cause the tensile stress and lead to the propagation of the crack in the electrolyte. If CTE of cathode is less than the ones of anode and electrolyte, the propagation of the blister crack in the interface will be possible. The expressions of Y for blister crack (case (e)) and crack in electrolyte (case (f)) are also given in the Appendix. C.

In the local and global failure criterions mentioned above, the failure strength and fracture toughness, allowable warpage, and allowable curvature are be considered as the deterministic values. In reality, however, the "strength" such as the failure strength, fracture toughness, and allowable warpage, and allowable curvature can be random variables with certain distributions, such as normal distribution, Weibull distribution, etc. In this case, the stress and strength discussed above should be considered as the average (mean) values and the failure criteria can only be stated as the probability of failure.

To illustrate how the statistical nature of the stresses and strength should be considered in the failure criteria, let $g(\sigma)$ and $g_{f}(\sigma)$ be the stress and strength distribution, respectively. The probability of failure at a given stress $\sigma$ is given by

$$
p_{f}=\int_{-\infty}^{\sigma} g_{f}(x) d x .
$$

For a given stress distribution $g(\sigma)$, the probability of failure is thus given by 


$$
p_{f}=\int_{-\infty}^{\infty} g(\sigma)\left[\int_{-\infty}^{\sigma} g_{f}(x) d x\right] d \sigma
$$

As an example, let's assume that both the stress and strength can be described by normal distributions, e.g., see Figure 2-52,

$$
\begin{aligned}
& g(\sigma)=\frac{1}{s \sqrt{2 \pi}} \exp \left[-\frac{1}{2}\left(\frac{\sigma-\bar{\sigma}}{s}\right)^{2}\right] \\
& g_{f}(\sigma)=\frac{1}{s_{f} \sqrt{2 \pi}} \exp \left[-\frac{1}{2}\left(\frac{\sigma-\bar{\sigma}_{f}}{s_{f}}\right)^{2}\right]
\end{aligned}
$$

where $s$ and $\bar{\sigma}$ represent standard deviation and mean value, respectively.

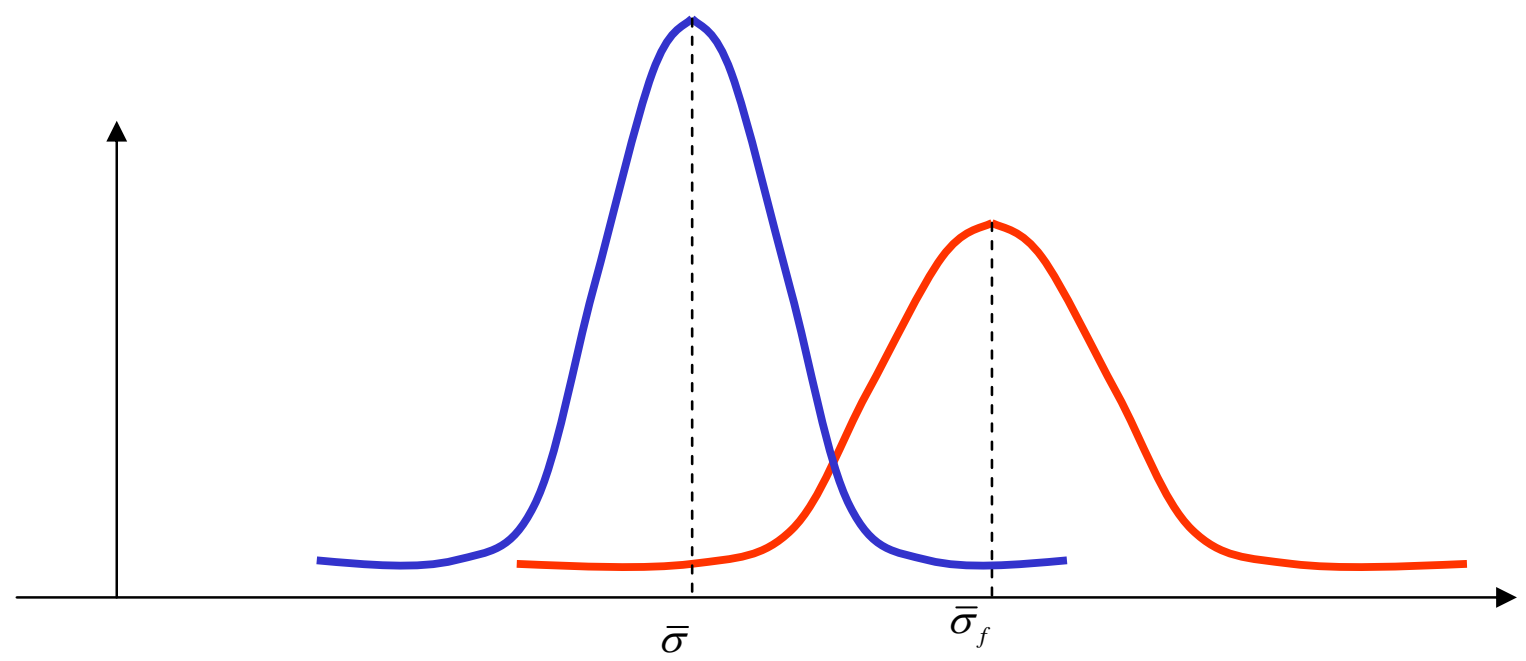

Figure 2-52: Illustration of normal distributions of stress and strength

For such given distributions, the probability of failure, according to Eq. (2.153) is given by

$$
p_{f}=\frac{1}{2 s \sqrt{2 \pi}} \int_{-\infty}^{\infty} \operatorname{Exp}\left[-\left(\frac{\sigma-\bar{\sigma}}{s \sqrt{2}}\right)^{2}\right] \operatorname{Erfc}\left[\frac{\bar{\sigma}_{f}-\sigma}{s_{f} \sqrt{2}}\right] d \sigma
$$

To see how the mean and deviation affect the probability of failure, Eq. (2.156) was evaluated for several different cases and the results are shown in Table 2-15. It is seen that the most critical parameter is the deviation. If the material strength or stress has large deviation, a large factor of safety must be used to ensure reliability.

Table 2-15: Probability of failure

\begin{tabular}{|l|l|l|}
\hline Factor of safety & Deviation & Failure probability \\
$\bar{\sigma}_{f} / \bar{\sigma}$ & $s / \bar{\sigma}_{f}=s_{f} / \bar{\sigma}_{f}$ & $p_{f}$ \\
\hline
\end{tabular}




\begin{tabular}{|l|l|l|}
\hline 1.0 & Any value & 0.5 \\
\hline 2.0 & 0.2 & $3.8 \times 10^{-2}$ \\
\hline 5.0 & 0.2 & $2.3 \times 10^{-4}$ \\
\hline 10.0 & 0.2 & $7.3 \times 10^{-5}$ \\
\hline 1.5 & 0.1 & $9.2 \times 10^{-3}$ \\
\hline 2.0 & 0.1 & $2.0 \times 10^{-4}$ \\
\hline 3.0 & 0.1 & $1.2 \times 10^{-6}$ \\
\hline 4.0 & 0.1 & $5.7 \times 10^{-8}$ \\
\hline 1.5 & 0.5 & $1.2 \times 10^{-6}$ \\
\hline 2.0 & 0.5 & $7.7 \times 10^{-13}$ \\
\hline 1.5 & 0.02 & $2.3 \times 10^{-32}$ \\
\hline
\end{tabular}

\subsection{Thermal Shock induced Failure of Anode Materials}

This is an initial investigation analysis of the thermal-shock test data conducted by ORNL on YSZ/Ni cermets. The analysis is further improved in this report by including the temperature-independent properties.

The temperature dependent Young's modulus of the sample material is shown in Figure 2-53. The temperature dependency of the Poisson's ratio and the CTE is neglected. Their values are taken as 0.25 and $11.8 \times 10^{-6}$, respectively.

To conduct statistical analysis of the data, the thermal shock tests conducted by ORNL are simulated numerically using the finite element method. The geometry of the samples used in the thermal shock test is illustrated in Figure 2-54.

In the thermal shock test, two batches of samples were used. In this report, they will be named Exp 1 and Exp 2, respectively. Each batch has 17 samples. The temperature distributions during the test were recorded for each sample just before the sample failed. Figure 2-55 shows the typical temperature distribution before fracture failure of the samples for the two batches, respectively. 


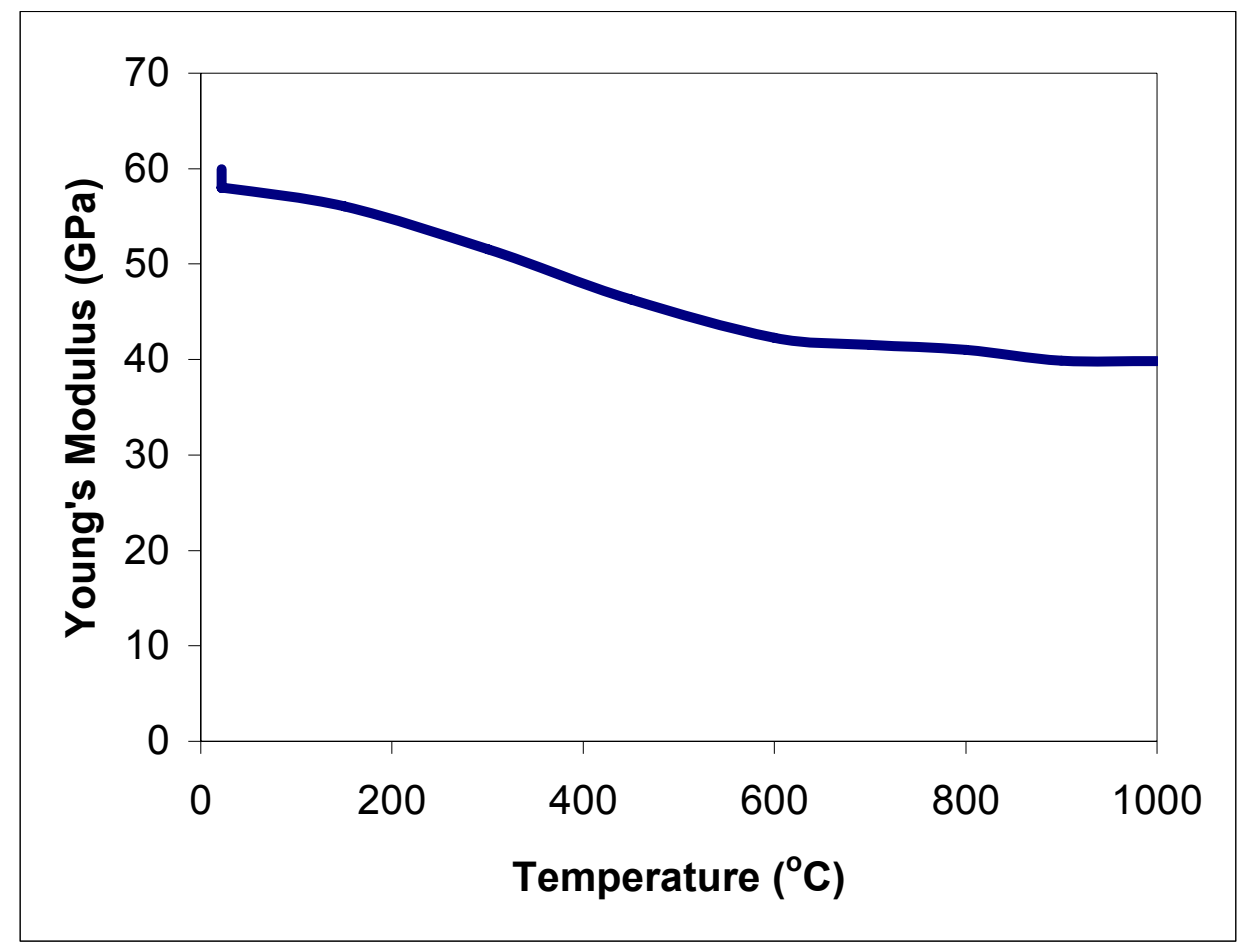

Figure 2-53: Curve of Young's modulus over temperature

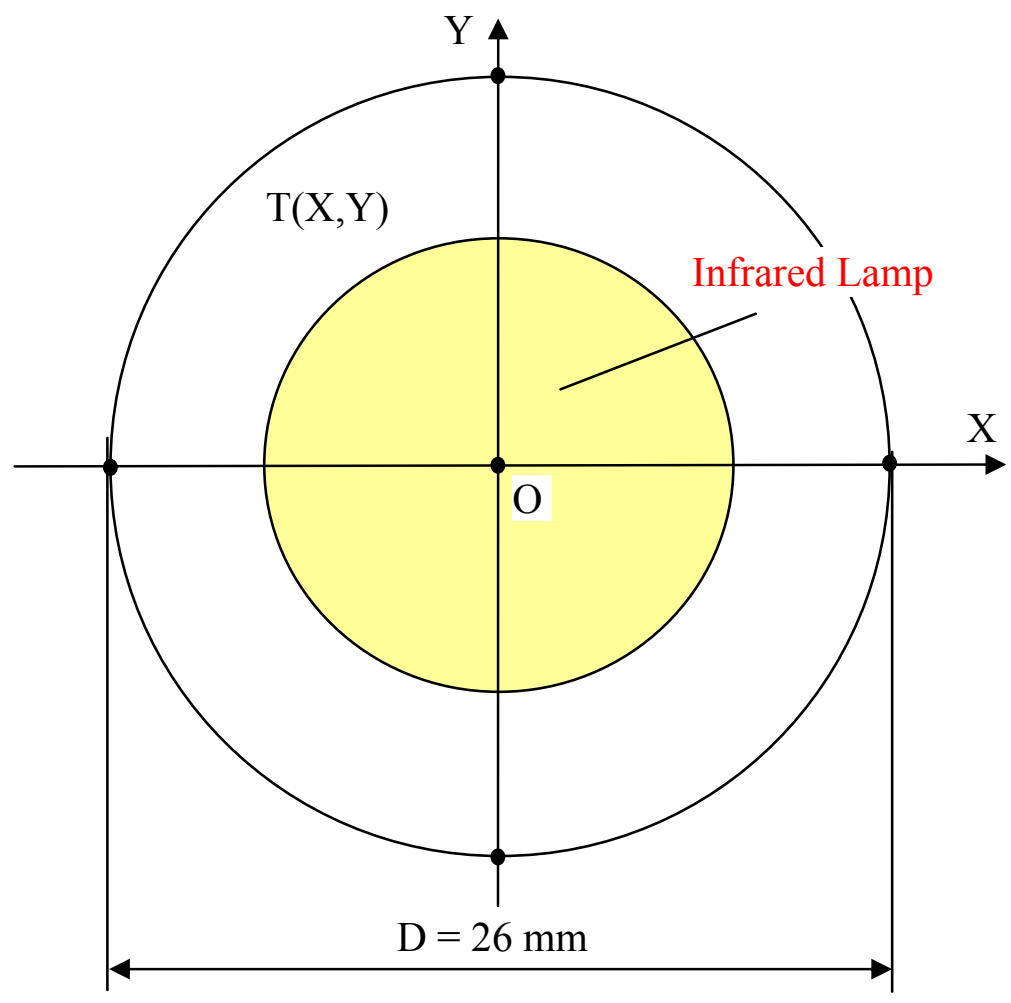

Figure 2-54: Illustration of geometry of sample used in thermal shock test 


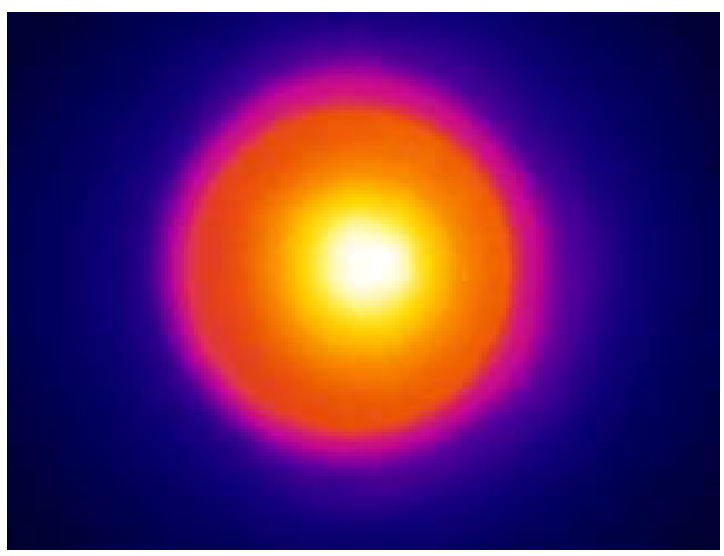

Exp 1: 03-11

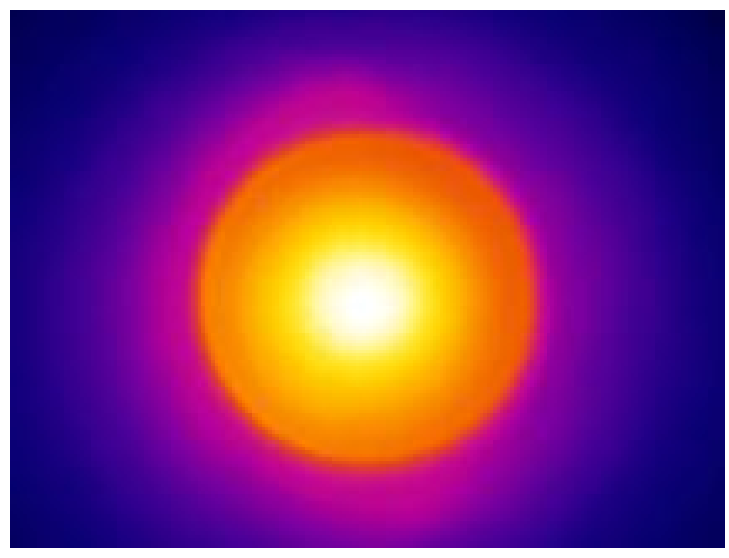

Exp 2: 06-09

Figure 2-55: Temperature field before just before the sample failed

Compared with the diameter, the thickness of the samples is very small. Therefore, the deformation may be considered as plane stress. Due to various factors during the test, the temperature field is not exactly axial-symmetric. Coupled thermalmechanical elements for 2D plane stress were used in the numerical analysis. The finite element mesh is shown in Figure 2-56. Figure 2-57 through Figure 2-58 show the nonsymmetric stress distribution. The unit used here for the stress is kilopascal.

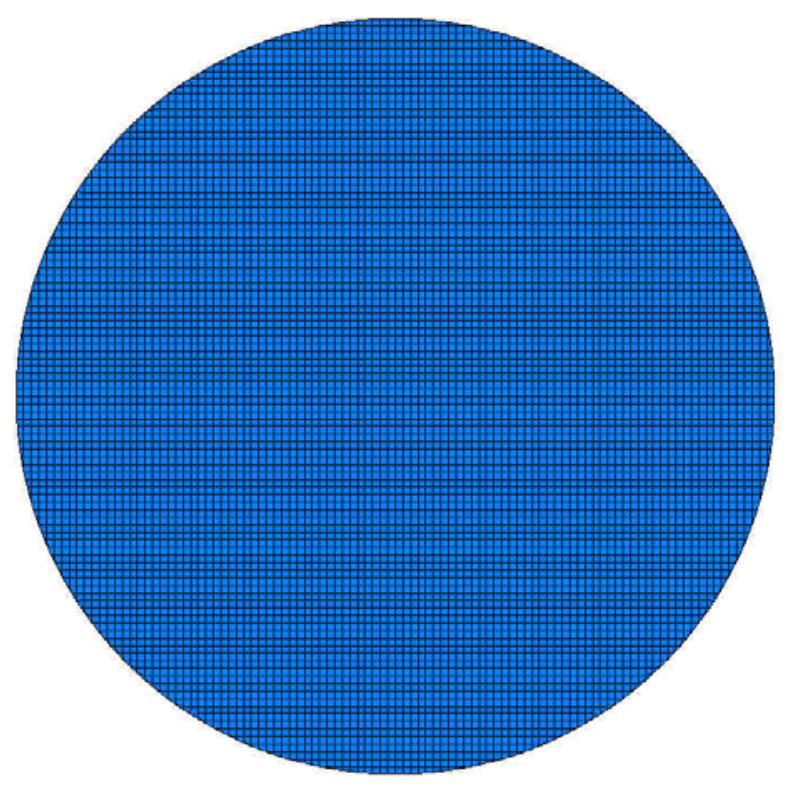

Figure 2-56: Finite element mesh 


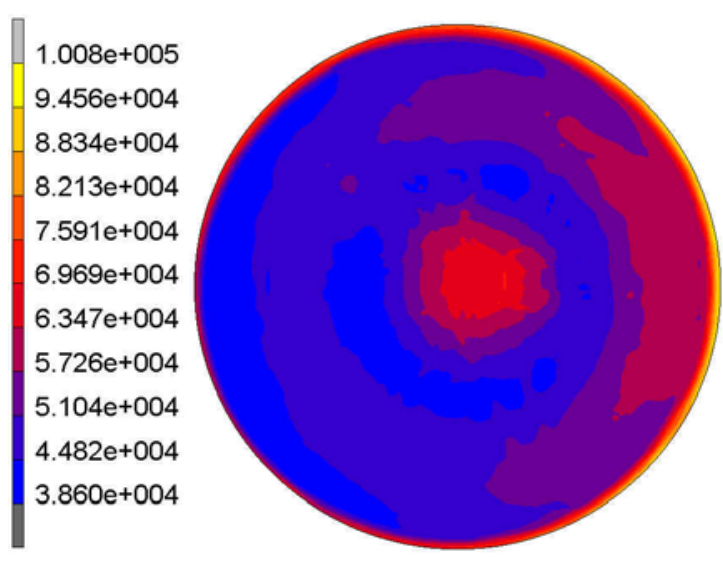

Von Mises Stress
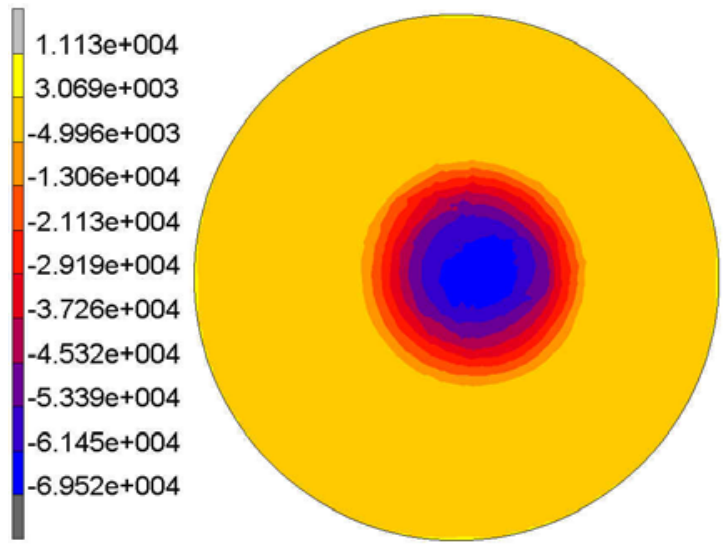

Intermediate Principal Stress

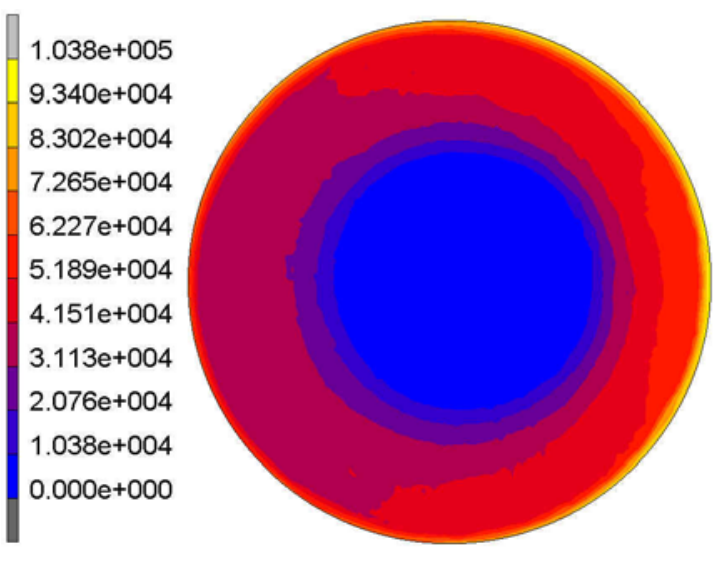

Maximum Principal Stress
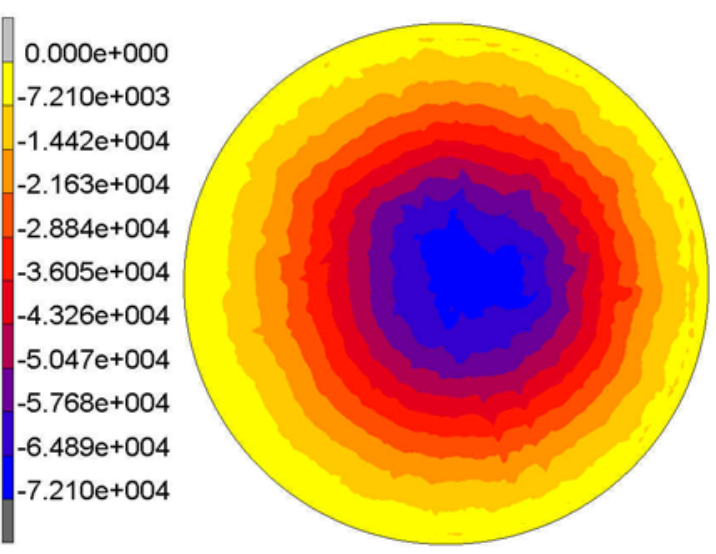

Minimum Principal Stress

Figure 2-57: Stress distribution obtained by FEM for Exp 1: 03-11

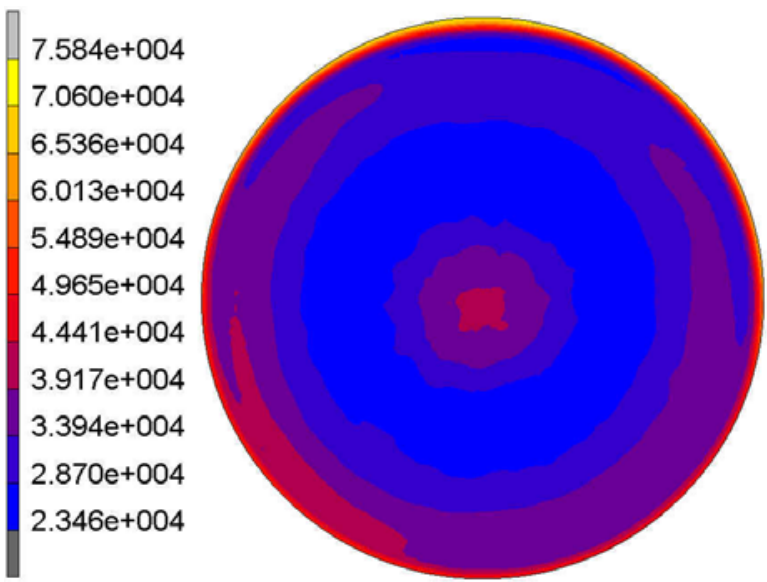

Von Mises Stress

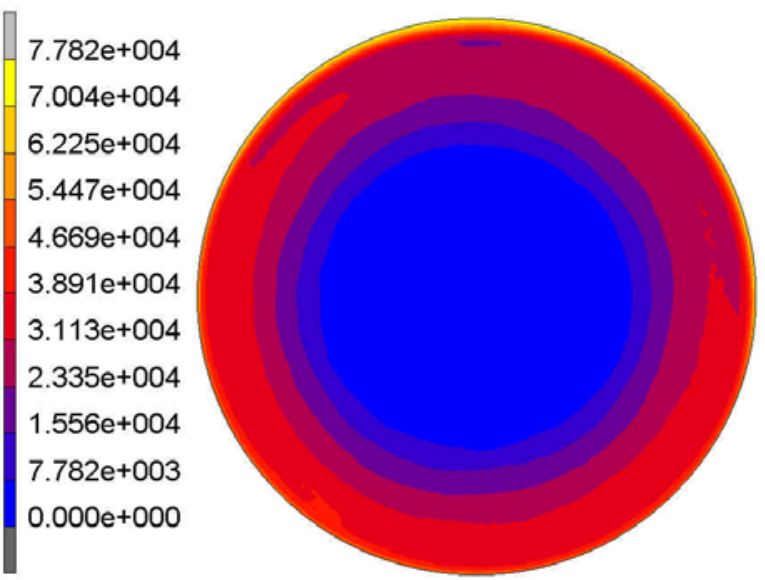

Maximum Principal Stress 


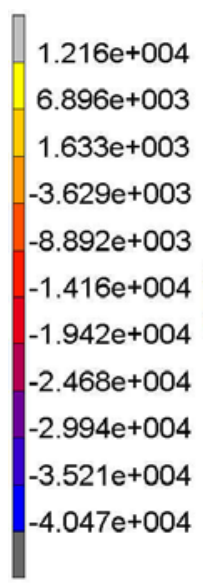

Intermediate Principal Stress

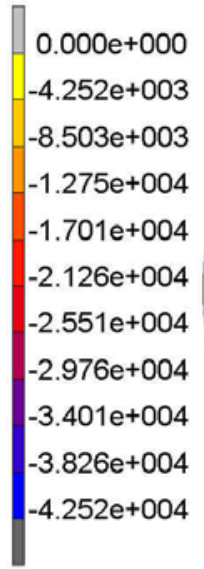

Minimum Principal Stress

Figure 2-58: Stress distribution obtained by FEM for Exp 2: 06-09

Now, consider a sample under stress. Let's assume the sample can be divided into $n$ elements spatially. We assume that the failure of any element leads to the failure of the entire sample. Let the probability of failure of the $\mathrm{i}^{\text {th }}$ element be $\Delta P_{f, i}$. Then, the probability of survival $P_{s}$ of this element is

$$
P_{s, i}=1-\Delta P_{f, i}
$$

Since the divided elements are independent and mutually exclusive, the resultant probability of survival of the entire sample under test is the product of the individual probability of survival of each element,

$$
P_{s}=\prod_{i=1}^{n}\left(1-\Delta P_{f, i}\right) \cong \exp \left(-\sum_{i=1}^{n} \Delta P_{f, i}\right)
$$

Next, we define the failure probability for a ceramic material of unit volume under a stress $\sigma$ to be $N_{V}(\sigma)$. Then the probability of failure of a representative volume $\Delta V$ under the stress, $\sigma$, is given by

$$
\Delta P_{f}=N_{V}(\sigma) \Delta V
$$

If the total volume of the sample is $V$, and the stress distribution within $V$ is given by $\sigma$, then, the probability of survival of this sample would be

$$
P_{s}=\exp \left[-\int_{V} N_{V}(\sigma) d V\right]
$$

The failure probability of the sample is then given by

$$
P_{f}=1-\exp \left[-\int_{V} N_{V}(\sigma) d V\right]
$$

For the Weibull two-parameter distribution, the function, $N_{V}(\sigma)$, is defined as 


$$
N_{V}(\sigma)=\left(\frac{\sigma}{\sigma_{o V}}\right)^{m_{V}}
$$

where the scale parameter $\sigma_{o V}$ corresponds to the stress level where 63.2 percent of the specimens with unit volume would fail, and $m_{V}$ is the shape parameter called Weibull modulus, a dimensionless parameter that measures the degree of strength variability.

For multi-axial stress states, each principal stress is treated as an independent driving force. Thus, for all $\sigma_{1} \geq \sigma_{2} \geq \sigma_{3}>0$, the probability of failure is

$$
P_{f}=1-\exp \left[-\int_{V}\left(N_{V}\left(\sigma_{1}\right)+N_{V}\left(\sigma_{2}\right)+N_{V}\left(\sigma_{3}\right)\right) d V\right]
$$

The strength distribution of Ni-YSZ cermet is determined by ORNL by means of Ring-on-ring test, as shown in Figure 2-59.

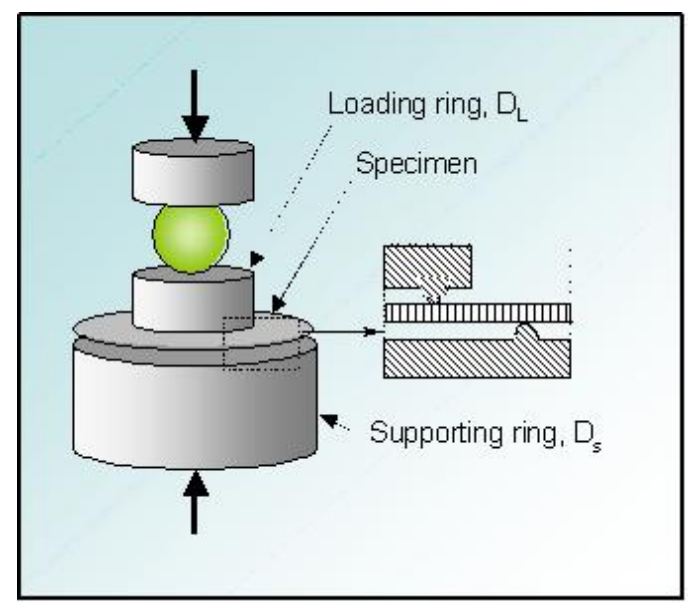

Figure 2-59: Schematic ring-on-ring test ${ }^{3}$

The equibiaxial strength is obtained as

$$
\sigma_{f}=\frac{3 F}{2 \pi h^{2}}\left[(1-v) \frac{D_{S}^{2}-D_{L}^{2}}{2 D^{2}}+(1+v) \ln \frac{D_{S}}{D_{L}}\right]
$$

The effective tensile surface area can be estimated by

$$
A e \cong \frac{\pi}{2} D_{L}^{2}\left\{1+\frac{44(1+v)}{3\left(1+m_{V}\right)} \frac{5+m_{V}}{2+m_{V}}\left(\frac{D_{S}-D_{L}}{D_{S} D}\right)^{2}\left[\frac{2 D^{2}(1+v)+\left(D_{S}-D_{L}\right)^{2}(1-v)}{(3+v)(1+3 v)}\right]\right\}
$$

For the sample with $39 \%$ of porosity, the parameters for Weibull distribution are obtained by ORNL and are given in Table 2-16. For any given temperature, the respective parameter will be calculated by linear interpolation as

\footnotetext{
${ }^{3}$ http://www.seca.doe.gov/ secamat/
} 


$$
f(T)=\frac{f(T=600)-f(T=22)}{600-22}(T-22)+f(T=22)
$$

Table 2-16: Parameters of strength distribution in Weibull analysis

\begin{tabular}{|c|c|c|}
\hline & $\begin{array}{c}\text { Characteristic } \\
\text { strength }(\mathrm{MPa})\end{array}$ & $\begin{array}{c}\text { Weibull } \\
\text { Modulus }\end{array}$ \\
\hline At $22^{\circ} \mathrm{C}$ & 55.42 & 4.49 \\
\hline At $600^{\circ} \mathrm{C}$ & 78.95 & 5.61 \\
\hline
\end{tabular}

For the normal distribution, the function $N_{V}(\sigma)$ is expressed as

$$
N_{V}(\sigma)=\frac{1}{s_{f} \sqrt{2 \pi}} \int_{0}^{\sigma} \exp \left[-\frac{1}{2}\left(\frac{x-\bar{\sigma}_{f}}{s_{f}}\right)^{2}\right] d x
$$

The corresponding parameter obtained by ORNL is included in Table 2-17

Table 2-17: Parameters of strength distribution in Normal distribution

\begin{tabular}{|c|c|c|}
\hline & $\begin{array}{c}\text { Average Strength } \\
(\mathrm{MPa})\end{array}$ & $\begin{array}{c}\text { Standard } \\
\text { Deviation }\end{array}$ \\
\hline At $22^{\circ} \mathrm{C}$ & 50.73 & 12.00 \\
\hline At $600^{\circ} \mathrm{C}$ & 72.92 & 15.02 \\
\hline
\end{tabular}

Based on the function $N_{V}(\sigma)$ and strength the distributions from the ring-on-ring tests, the resultant probability of failure may be calculated as

$$
P_{f}=1-\exp \left[-\frac{1}{A e} \int_{V} N_{V}(\sigma) d V\right]
$$

The results are shown in Table 2-18.

Table 2-18: Comparison between the predicted failure rate and the actual test data

\begin{tabular}{|c|c|c|c|c|c|}
\hline \multirow{2}{*}{} & \multirow{2}{*}{ Experiment } & \multicolumn{4}{|c|}{ Prediction } \\
\cline { 3 - 6 } & & \multicolumn{2}{|c|}{ Principal Stresses } & \multicolumn{2}{|c|}{ Von Mises Stress } \\
\cline { 3 - 6 } & Weibull & Normal & Weibull & Normal \\
\hline Exp 1 & $80 \%$ & $82 \%$ & $77 \%$ & $96 \%$ & $96 \%$ \\
\hline Exp 2 & $41 \%$ & $63 \%$ & $43 \%$ & $65 \%$ & $50 \%$ \\
\hline
\end{tabular}

It can be seen from the above results that the Mises stress criterion predicts much high failure rate than that predicted by the principal stress criterion. In comparison with the experimental data, the failure rates predicted by the principle stress criterion are much closer to the actual tested data. Therefore, we recommend that the principal stress criterion should be used for the YSZ/Ni cermets under thermal shock conditions. Further 
more, it appears that normal distribution is a better fit of the actual test data than the Weibull distribution, as least for the second batch of samples (Exp 2).

It should also be pointed out that the statistical strength used here is not for exactly the same material under the thermal shock tests analyzed here. ORNL is in the process of collecting the strength data. Analysis will be conducted when that data becomes available.

\subsection{Develop models for thermal shock-induced failure in SOFC}

During the start-up process, temperature gradient is created in the cell by the flow of heated air in channels. Such a temperature gradient is closely related to the heating rate of the air and flow velocity in the channel. The temperature gradient in cell typically causes stress, which in tern may cause the initiation and propagation of cracks and delamination. This section will study the stress caused by the heated air in the channel is investigated based on the non-uniform temperature obtained by the second-order model from the startup to steady state, and the effect of the heating rate and flow velocity on the stress is investigated.

The temperature distribution along the channel is expressed as

$$
\begin{aligned}
& T(z, t)=\theta^{*}(z, t)+\frac{4+2 P e-2 P e \cdot z}{(4+P e)}(K t) e^{\frac{P e}{2} z} \\
& \theta^{*}(z, t)=\sum_{n=1}^{\infty} C_{n}(t)\left[\sin \left(\omega_{n} z\right)+\frac{2 \omega_{n}}{P e} \cos \left(\omega_{n} z\right)\right] e^{\frac{P e}{2} z} \\
& C_{n}(t)=-\frac{2 K}{G_{n} \omega\left(4 \lambda_{n}^{2}+P e\right)^{2}}\left[\left(16 \lambda_{n}^{2}+P e^{2} t+4 P e \lambda_{n}^{2} t\right) e^{\frac{P e}{4} t}-16 \lambda_{n}^{2} e^{-\lambda_{n}^{2} t}\right]
\end{aligned}
$$

where

$$
\begin{aligned}
& K_{e f f}=\frac{K^{*} L}{u_{e f f}\left(T_{f}-T_{o}\right)} \\
& \frac{1}{P e}=\frac{\alpha_{e f f}}{u_{e f f} L}=\frac{\text { diffusion of thermal energy }}{\text { advection of thermal energy }} \\
& T=\frac{T^{*}-T_{o}}{T_{f}-T_{o}} ; \quad z=\frac{z^{*}}{L} ; \quad t=\frac{t^{*}}{\left(L / u_{e f f}\right)} \\
& u_{e f f}=\frac{\left(\rho c_{p} A\right)_{g}}{\left(\rho c_{p} A\right)_{I C_{1}}+\left(\rho c_{p} A\right)_{g}+\left(\rho c_{p} A\right)_{C}+\left(\rho c_{p} A\right)_{E}+\left(\rho c_{p} A\right)_{A}+\left(\rho c_{p} A\right)_{f}+\left(\rho c_{p} A\right)_{I C_{2}}} \cdot u \\
& \alpha_{e f f}=\frac{(k A)_{I C_{1}}+(k A)_{g}+(k A)_{C}+(k A)_{E}+(k A)_{A}+(k A)_{f}+(k A)_{I C_{2}}}{\left(\rho c_{p} A\right)_{I C_{1}}+\left(\rho C_{p} A\right)_{g}+\left(\rho c_{p} A\right)_{C}+\left(\rho C_{p} A\right)_{E}+\left(\rho C_{p} A\right)_{A}+\left(\rho C_{p} A\right)_{f}+\left(\rho C_{p} A\right)_{I C_{2}}}
\end{aligned}
$$




$$
\sigma_{\text {eff }}=\frac{(k A)_{I C_{1}}+(k A)_{C}+(k A)_{E}+(k A)_{A}+(k A)_{f}+(k A)_{I C_{2}}}{\left(\rho c_{p} A\right)_{g} u}
$$

As an example, consider the following case, where the initial temperature of the cell and air is taken as room temperature, $25^{\circ} \mathrm{C}$. The temperature of the air starts from the room temperature gradually increases to the steady-state temperature of $625^{\circ} \mathrm{C}$ at the rate of $1.0{ }^{\circ} \mathrm{C} / \mathrm{sec}$. The air flow velocity at the inlet controlled at $10 \mathrm{~m} / \mathrm{sec}$. The corresponding temperature distribution along the channel is shown in Figure 2-60 at different time.

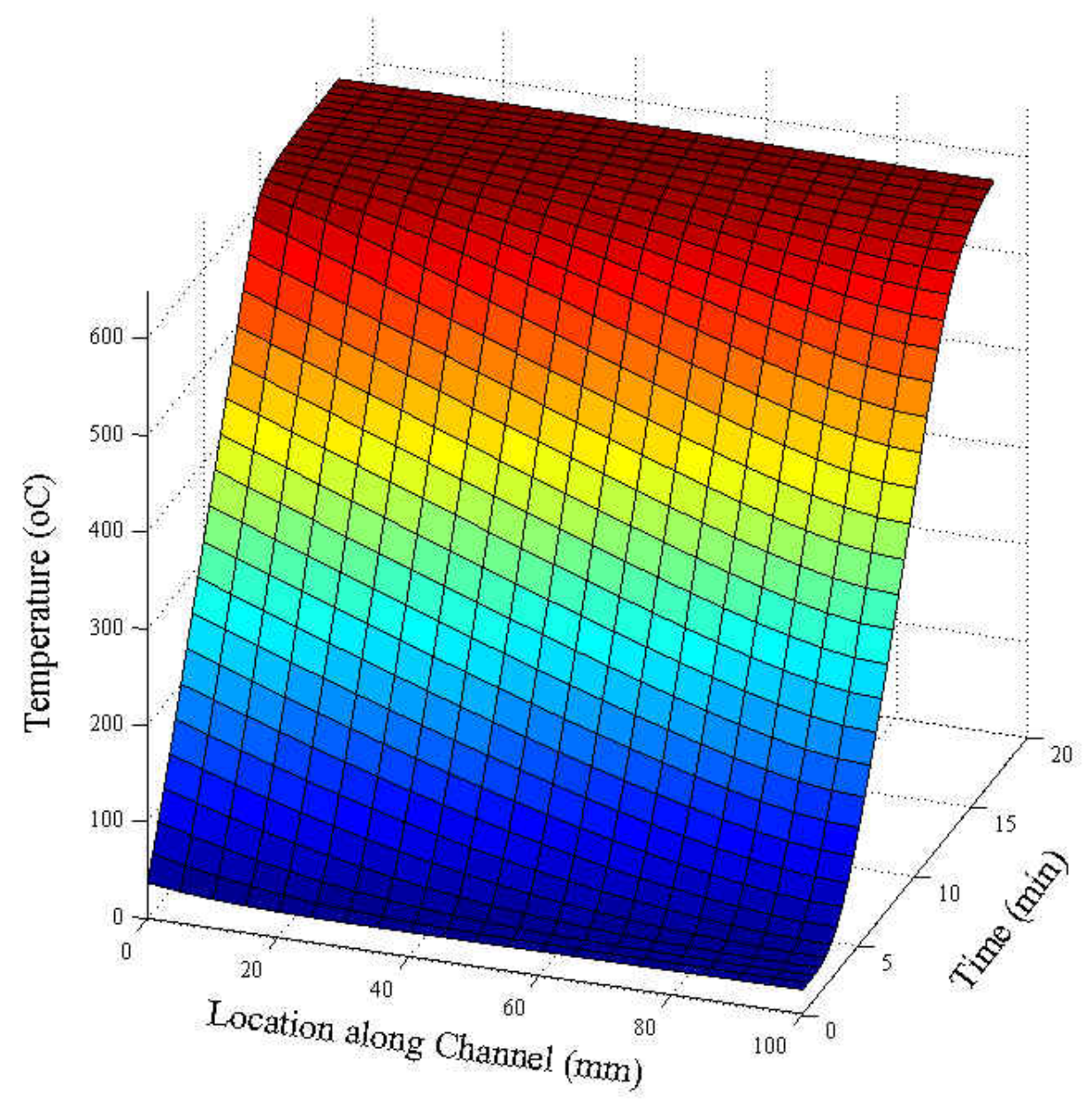

Figure 2-60: Temperature distribution along air channel

In the thermal analysis, the temperature along the thickness of the cell is assumed constant. For calculating the stress, we further assume that PEN structure is simulated by an axisymmetric temperature field in a half-space. This is certainly a rather gross assumption. But, it allows us the obtain an analytical solution to the stress field and provide certain insights of how the transient stress fields evolves during start-up. Under this assumption, the elastic strain due to temperature is obtained in the polar coordinate system 


$$
\begin{aligned}
& \varepsilon_{r}=\frac{1}{E}\left(\sigma_{r}-v \sigma_{\theta}\right)+\alpha T \\
& \varepsilon_{\theta}=\frac{1}{E}\left(\sigma_{\theta}-v \sigma_{r}\right)+\alpha T
\end{aligned}
$$

With the help of Hooke's law, $\sigma=E \varepsilon^{e}$, the above equations may be rewritten as

$$
\begin{aligned}
& \sigma_{r}=\frac{E}{1-v^{2}}\left[\varepsilon_{r}+v \varepsilon_{\theta}-(1-v) \alpha T\right] \\
& \sigma_{\theta}=\frac{E}{1-v^{2}}\left[\varepsilon_{\theta}+v \varepsilon_{r}-(1-v) \alpha T\right]
\end{aligned}
$$

After some simplification, the stresses are obtained as

$$
\begin{aligned}
& \sigma_{r}=\alpha E\left(\frac{1}{R^{2}} \int_{0}^{R} \operatorname{Trd} r-\frac{1}{r^{2}} \int_{0}^{r} \operatorname{Tr} d r\right) \\
& \sigma_{\theta}=\alpha E\left(\frac{1}{R^{2}} \int_{0}^{R} \operatorname{Tr} d r+\frac{1}{r^{2}} \int_{0}^{r} \operatorname{Tr} d r-T\right)
\end{aligned}
$$

To illustrate how the transient stress evolves, consider a numerical example based on the material properties listed in Table 2-19. Figure 2-61 and Figure 2-62 show the distribution of the stress for air flow velocity $1 \mathrm{~m} / \mathrm{s}$ with heating rate $0.1^{0} \mathrm{C} / \mathrm{s}$ and air flow velocity $10 \mathrm{~m} / \mathrm{s}$ with heating rate $1.0^{\circ} \mathrm{C} / \mathrm{s}$, respectively. Curve of maximal tensile stress over air flow velocity is illustrated in Figure 2-63 for at heating rate $0.5^{0} \mathrm{C} / \mathrm{s}$ and $0.8^{0} \mathrm{C} / \mathrm{s}$, respectively. Figure 2-64 show us the curve of maximal tensile stress over heating rate at air flow velocities $1 \mathrm{~m} / \mathrm{s}, 5 \mathrm{~m} / \mathrm{s}$ and $8 \mathrm{~m} / \mathrm{s}$, respectively.

It can be seen that the maximum stress appears at the middle of start-up process. During the start-up, the maximum tensile stress in the cell increases when temperature gradient increases as the heated air flowing through the channel, and the maximum tensile stress reaches the largest value when temperature gradient reaches its peak, and then, the maximum tensile stress starts to decrease when the temperature field is approaching steady state and temperature gradient disappears.

It is seen that the increasing the heating rate will always increase the maximum tensile stress in the cell. However, the dependency on the flow velocity is not monotonic. It appears from Figure 2-63 that for each heating rate, there is a particular flow velocity at which the stress is the highest. Away from this flow velocity, the maximum tensile stress actually is lower. This points out a need to optimize the heating rate and flow velocity in order to shorten the start-up time without damaging the cell.

Table 2-19: Material properties

\begin{tabular}{|c|c|c|}
\hline $\begin{array}{c}\text { Young's Modulus } \\
(\mathrm{GPa})\end{array}$ & Poisson's ratio & $\mathrm{CTE}\left(10^{-6} /{ }^{\circ} \mathrm{C}\right)$ \\
\hline 96 & 0.3 & 11.2 \\
\hline
\end{tabular}




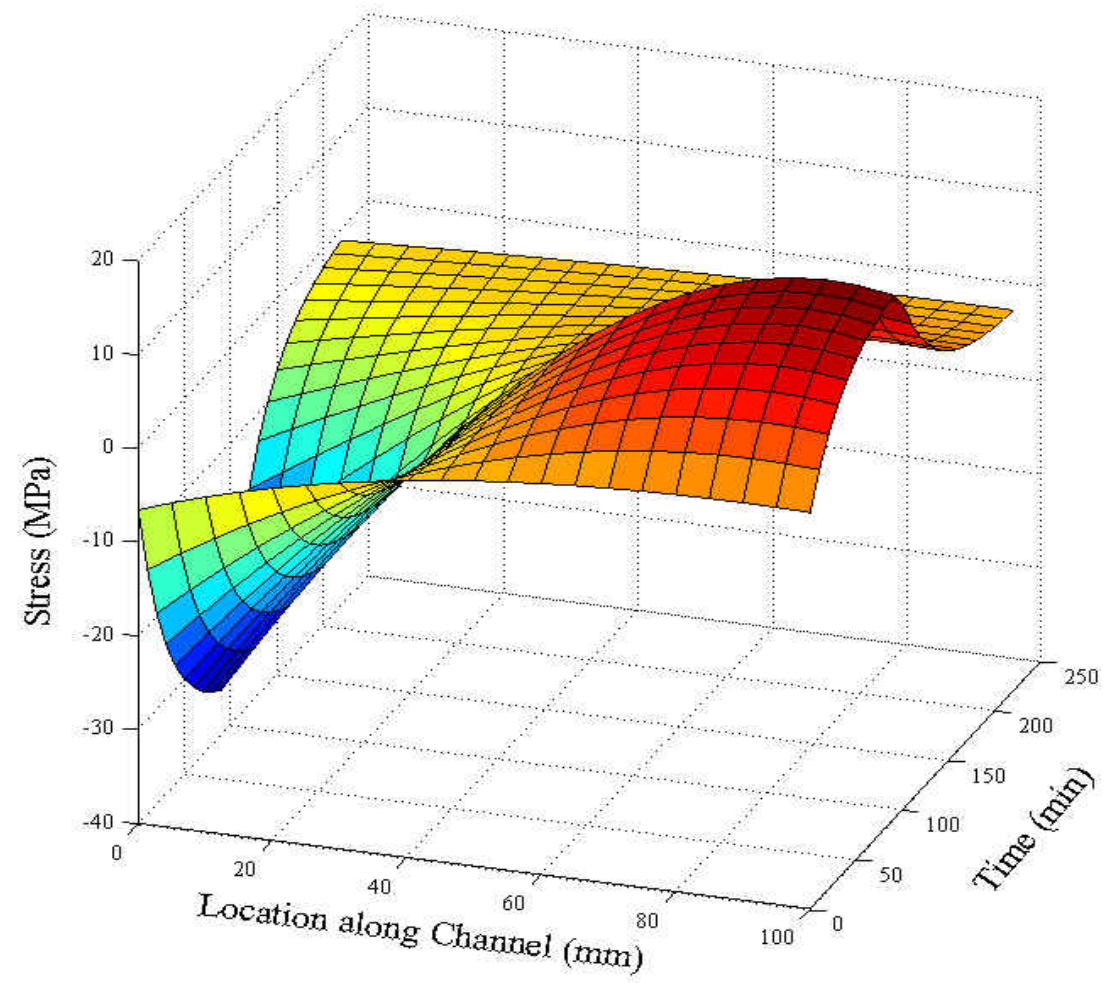

Figure 2-61: Distribution of stress: air flow velocity $1 \mathrm{~m} / \mathrm{s}$, heating rate $0.1^{\circ} \mathrm{C} / \mathrm{s}$ 


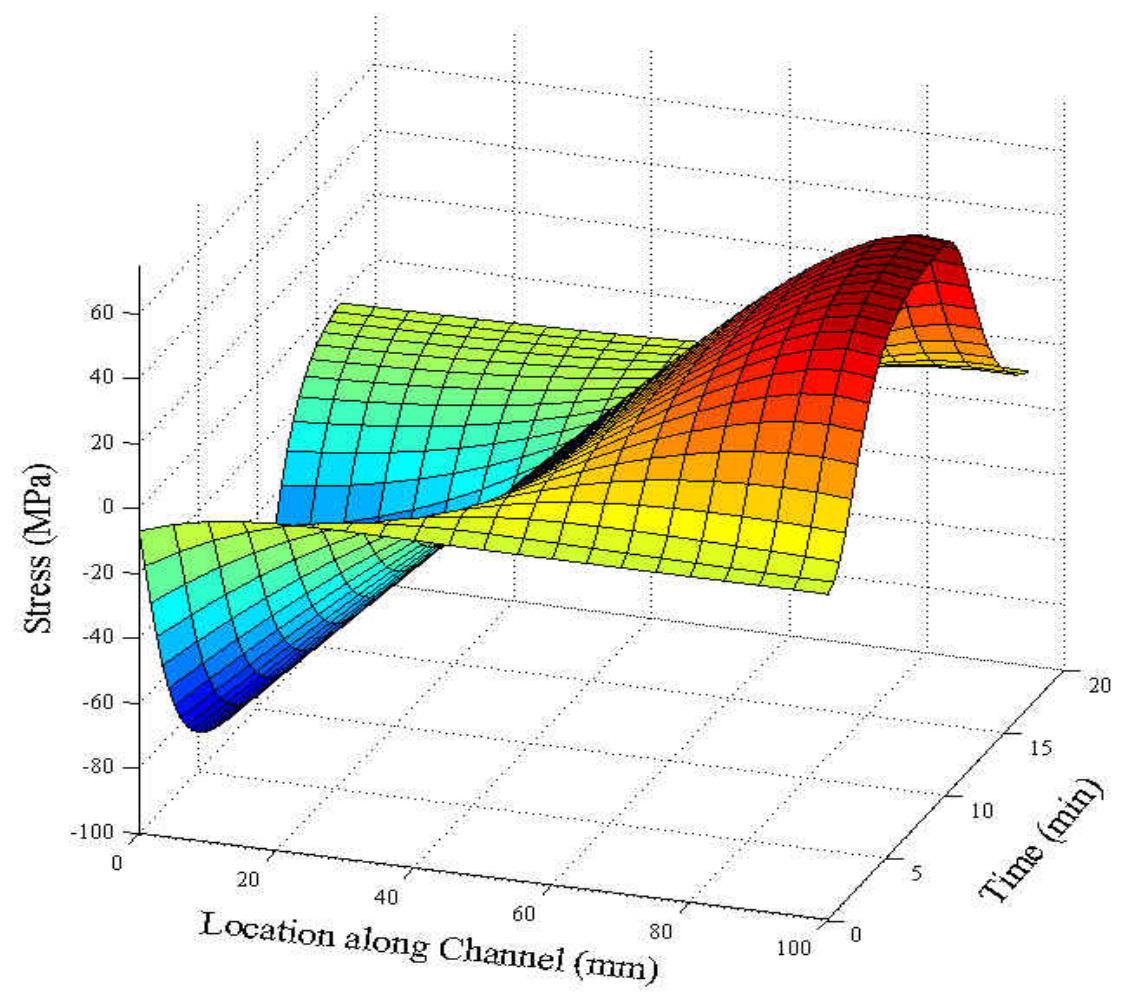

Figure 2-62: Distribution of stress: air flow velocity $10 \mathrm{~m} / \mathrm{s}$, heating rate $1.0^{\circ} \mathrm{C} / \mathrm{s}$

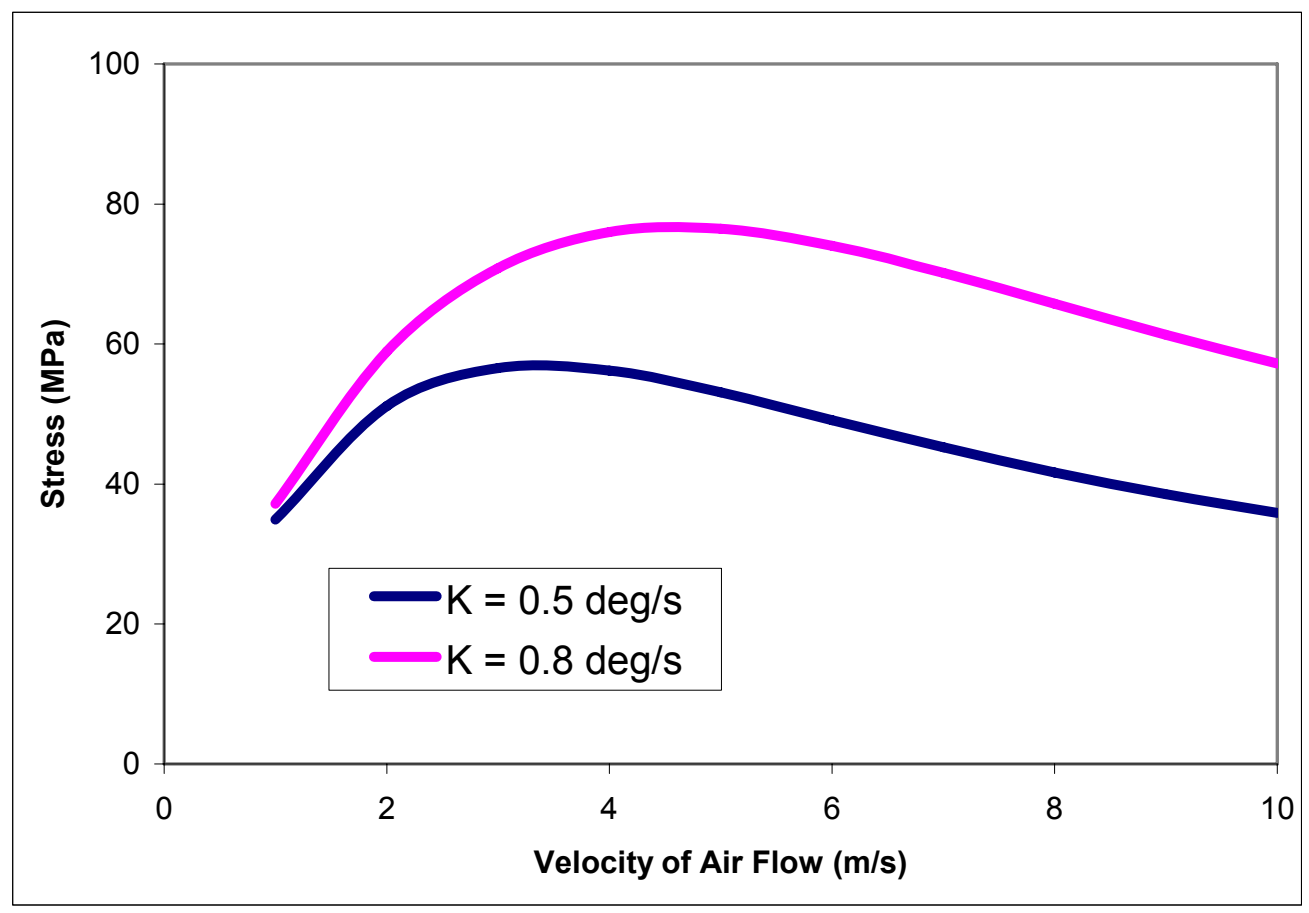

Figure 2-63: Curve of stress over air flow velocity at heating rate $0.5^{\circ} \mathrm{C} / \mathrm{s}$ and $0.8^{\circ} \mathrm{C} / \mathrm{s}$, respectively 


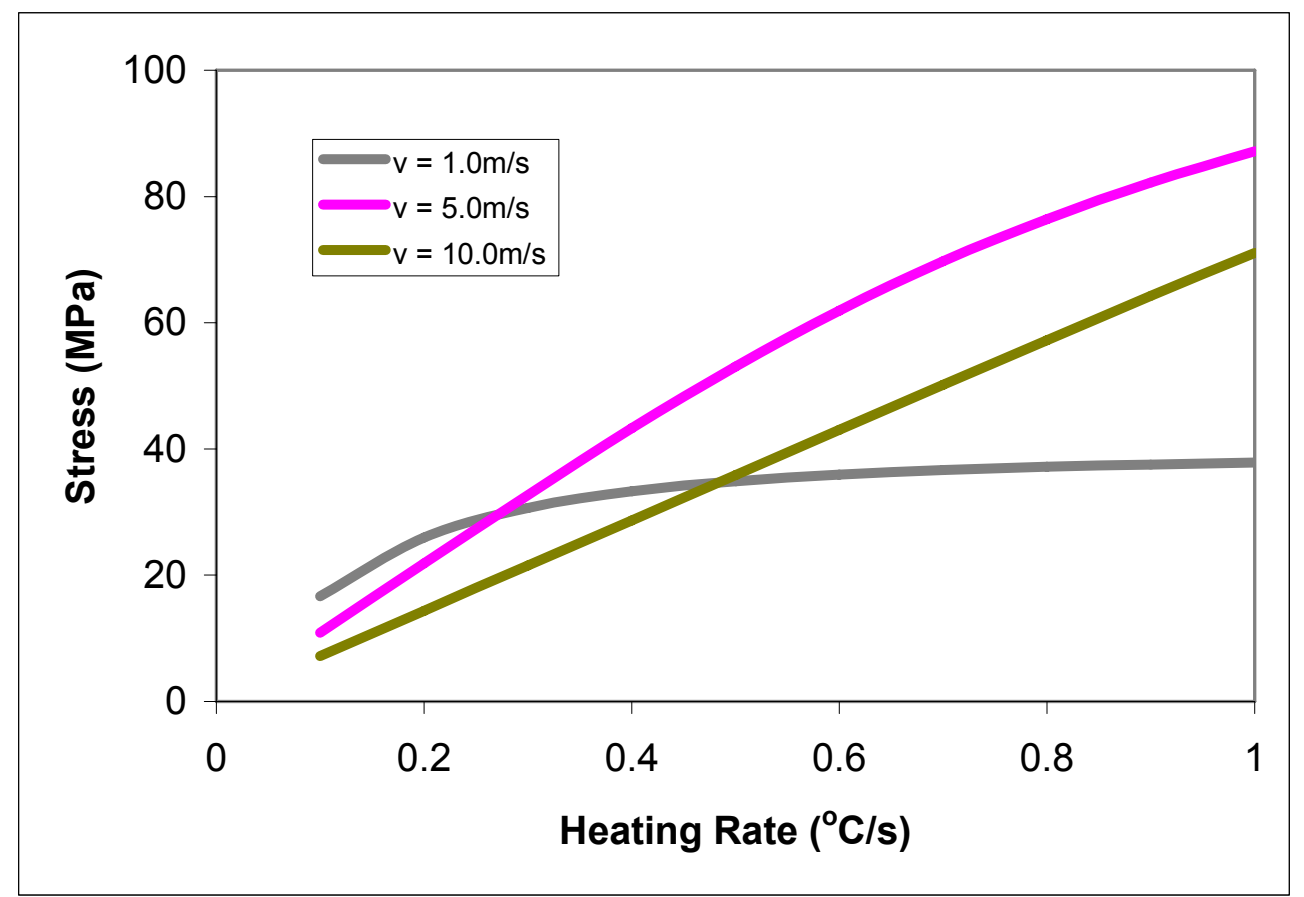

Figure 2-64: Curve of stress over heating rate at air flow velocity $1 \mathrm{~m} / \mathrm{s}, 5 \mathrm{~m} / \mathrm{s}$ and $8 \mathrm{~m} / \mathrm{s}$, respectively

\subsection{Develop models for thermal shock-induced failure in SOFC}

Previously, we have developed an analytical thermomechanical transient model to estimate the stresses in the PEN structure of a SOFC induced by thermal shock during start-up or shut-down. Based on this model, it was found that increasing the heating rate will always increase the maximum tensile stress in the cell. However, the dependency on the flow velocity is not monotonic. For each heating rate, there is a particular flow velocity at which the stress is the highest. Away from this flow velocity, the maximum tensile stress actually is lower. This points out a need to optimize the heating rate and flow velocity in order to shorten the start-up time without damaging the cell.

Because of the various assumptions made in developing the analytical model, predictions from this model needs to be verified. In this report, we present a comparison between the prediction of this model and that of a finite element solution using MARC with electrochemistry model developed at PNNL.

At the moment, MARC with the PNNL EC model can only analyze the case where the air temperature remains as a constant. This corresponds to infinite heating rate. In the case of infinite heating rate, our analytical model yields, 


$$
\begin{aligned}
& T(z, t)=\theta(z, t) \\
& \theta(z, t)=\theta^{*}(z, t)+\frac{4+2 P e-2 P e \cdot z}{(4+P e)} e^{\frac{P e}{2} z} \\
& \theta^{*}(z, t)=\sum_{n=1}^{\infty} C_{n}(t)\left[\sin \left(\omega_{n} z\right)+\frac{2 \omega_{n}}{P e} \cos \left(\omega_{n} z\right)\right] e^{\frac{P e}{2} z} \\
& C_{n}(t)=\frac{2}{G_{n} \omega}\left[-\left(J_{n}+M_{n}\right)\right] \\
& J_{n}=\frac{P e}{\left(4 \lambda_{n}^{2}+P e\right)}\left[1-e^{-\left(\lambda_{n}^{2}+\frac{P e}{4}\right) t}\right] \\
& M_{n}=4 e^{-\left(\lambda_{n}^{2}+\frac{P e}{4}\right) t}
\end{aligned}
$$

As an example, we consider the sudden cool down of a cell from $1073 \mathrm{~K}$ to $898 \mathrm{~K}$. This can be approximated by infinite cooling rate. Figure 2-65 shows the maximum stress vs. air flow velocity at infinite cooling rate. It is seen that the maximum tensile stress does not increase monotonically with the air flow velocity. There is a transition point $(\sim 18 \mathrm{~m} / \mathrm{s})$, above which the maximum tensile stress starts to decrease with increasing air flow velocity. This is consistent with our model predictions for the case when the heating rate is finite, as reported previously.

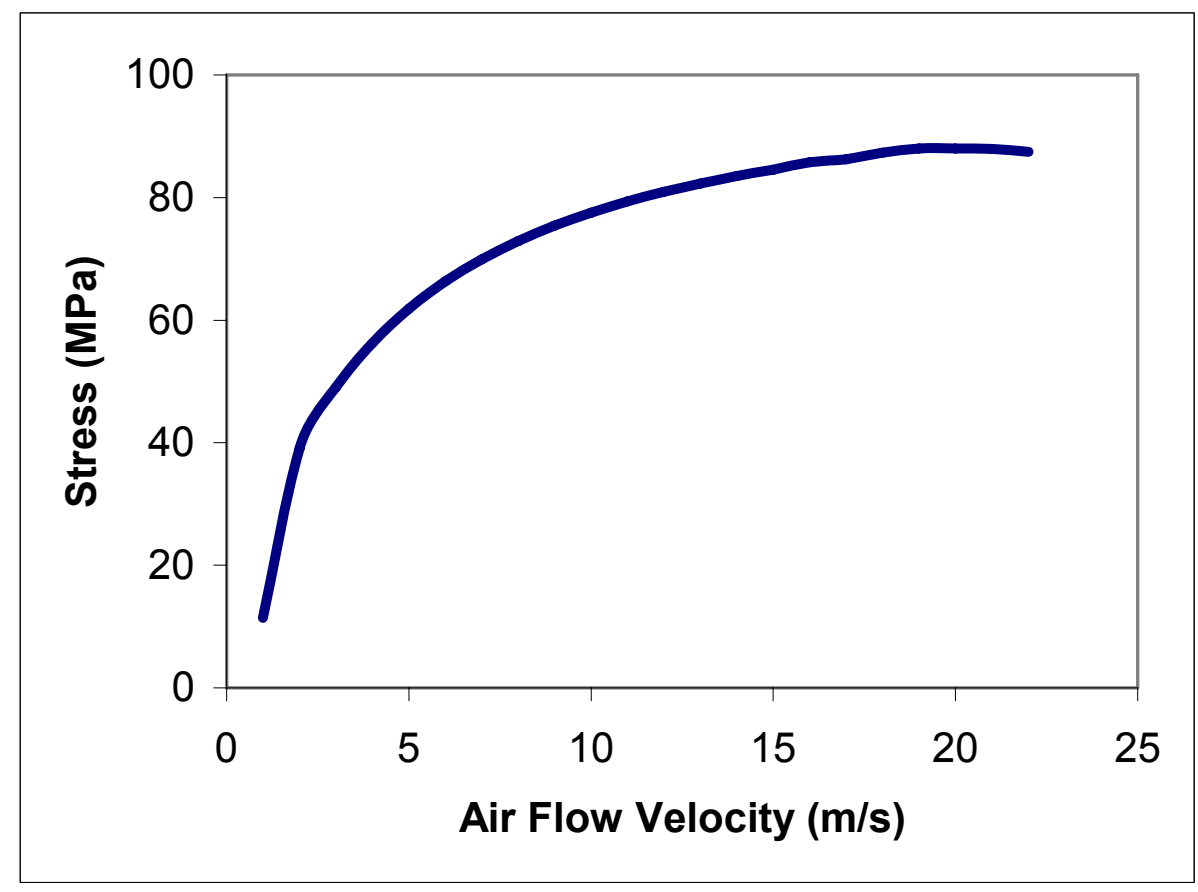

Figure 2-65: Curve of maximum tensile stress vs. air flow velocity with infinite cooling rate

Next, consider the same problem using the finite element code MARC. A single cell model defined by the default values in the MARC GUI EC presented by PNNL is used. 
The length, width, and thickness of the cell are $157.9 \mathrm{~mm}, 149.5 \mathrm{~mm}$, and $5.01 \mathrm{~mm}$, respectively. The finite element model is illustrated in Figure 2-66.
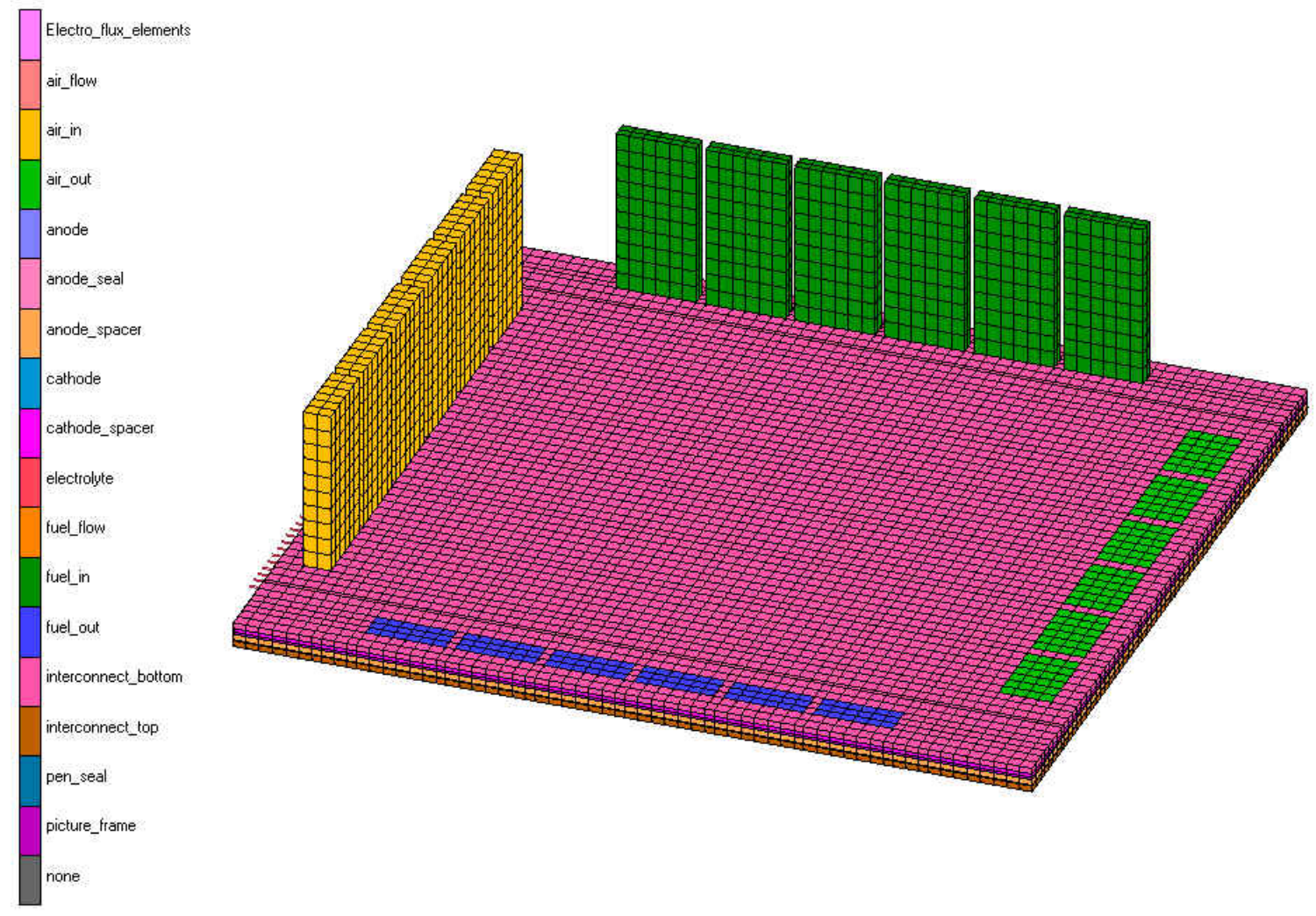

Figure 2-66: Illustration of finite element model of a single cell

In the EC model developed by PNNL, the Butler-Volmer relation is used. The electrolyte conductivity is expressed as

$$
k=A T^{3}+B T^{2}+C T+D
$$

with $\mathrm{A}=8.69 \mathrm{e}-8, \mathrm{~B}=-1.10 \mathrm{e}-4, \mathrm{C}=4.62 \mathrm{e}-2$, and $\mathrm{D}=-6.54$.

Table 2-20. Conductivity of anode, cathode, and interconnect

\begin{tabular}{|l|c|c|c|}
\hline & Anode & Cathode & Interconnect \\
\hline Conductivity & $10.0 \mathrm{e} 5$ & $5.76 \mathrm{e} 7$ & $8.8 \mathrm{e} 7$ \\
\hline
\end{tabular}

The exchange current is expressed as

$$
i=\gamma * e^{\frac{-W}{R T}}
$$

with $\gamma=8.29 \mathrm{e} 9$, and $W=1.3 \mathrm{e} 5$. For concentration polarization, the parameters are listed in Table 2-21. 
Table 2-21: Parameters for concentration polarization

\begin{tabular}{|l|l|l|}
\hline & anode & Cathode \\
\hline Porosity & 0.3 & 0.3 \\
\hline Tortuosity & 17 & 17 \\
\hline
\end{tabular}

The fuel and oxidant input parameters are listed in Table 2-22 below.

Table 2-22: Parameters for fuel and oxidant input

\begin{tabular}{|l|l|l|}
\hline & Fuel & Oxidant \\
\hline Pressure(atm) & 1.0 & 1.0 \\
\hline Temperature(K) & 898 & 898 \\
\hline File Coefficient & 1785.24 & 107.899 \\
\hline Effective Viscosity & $2.11319 \mathrm{e}-5$ & $4.46448 \mathrm{e}-5$ \\
\hline Density & 0.0336388 & 0.323574 \\
\hline Thermal Conductivity & 0.449992 & 0.0692296 \\
\hline Specific Heat & 15156.2 & 1117.88 \\
\hline \multirow{2}{*}{ Composition (molar \%) } & $\mathrm{H}_{2}: 0.97$ & $\mathrm{O}_{2}: 0.21$ \\
\cline { 2 - 3 } & $\mathrm{H}_{2} \mathrm{O}: 0.03$ & $\mathrm{~N}_{2}: 0.79$ \\
\hline
\end{tabular}

Boundary conditions are given in Table 2-23.

Table 2-23: Boundary conditions

\begin{tabular}{|l|l|l|}
\hline & Fuel & Oxidant \\
\hline Flow rate & $4.236 \mathrm{e}-4$ & $1.69 \mathrm{e}-2$ \\
\hline Inlet Temperature & 898 & 898 \\
\hline
\end{tabular}

Initial temperature of the cell/stack is $1073 \mathrm{~K}$.

The material properties are temperature dependent. The dependency of the Young's modulus and CTE on temperature is depicted in Figure 2-67 and Figure 2-68, respectively. 


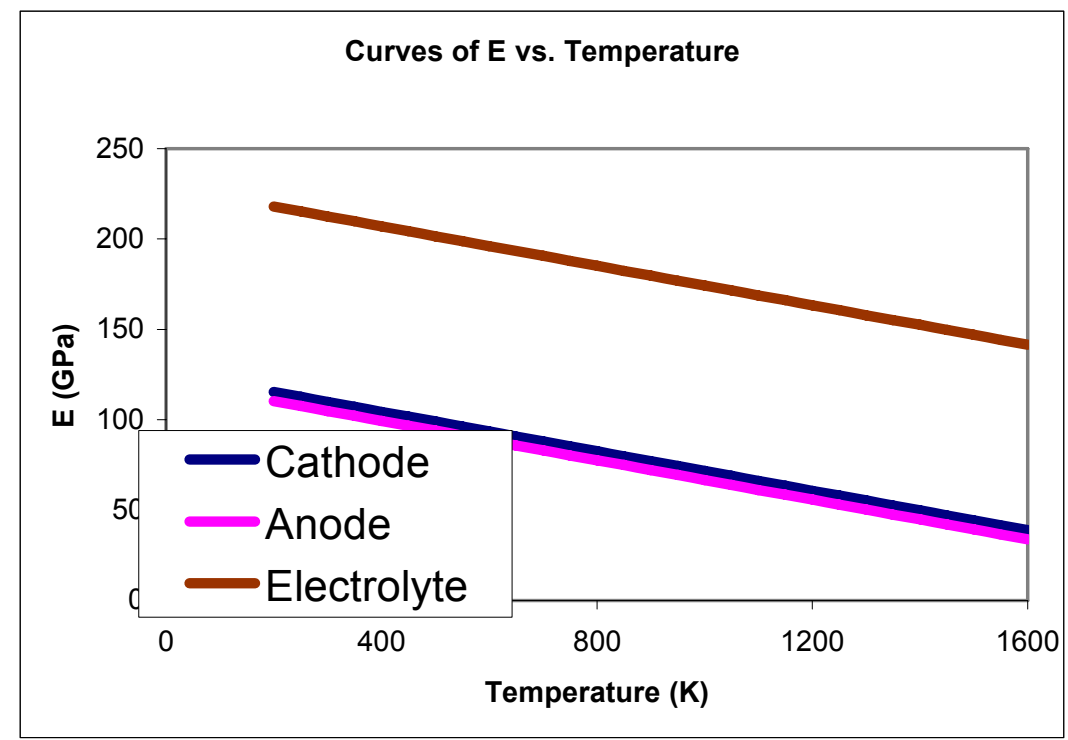

Figure 2-67: Temperature dependent Young's modulus

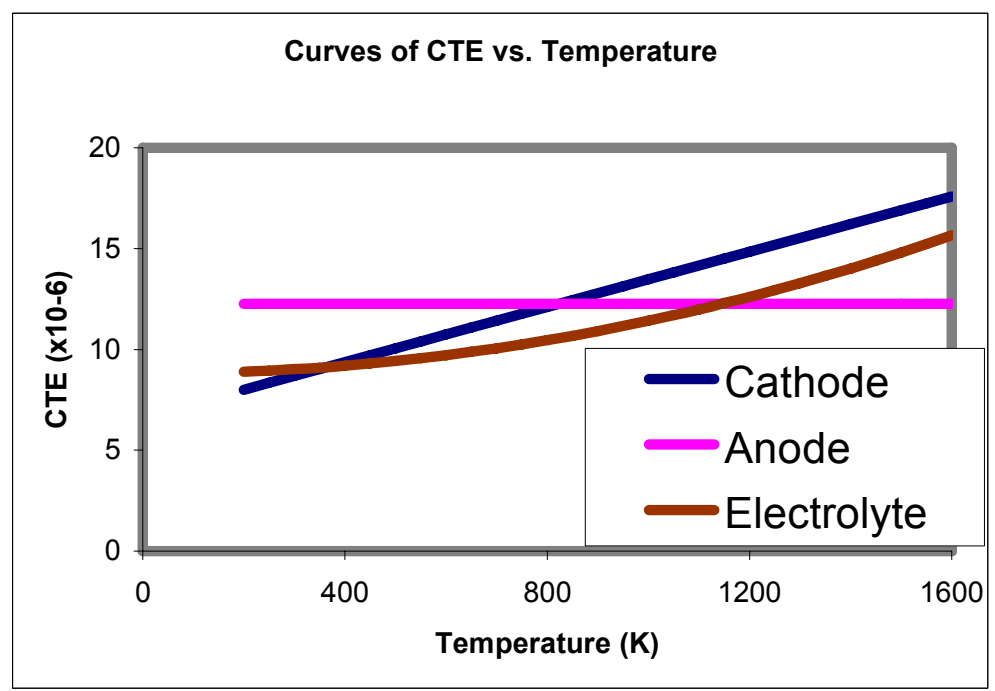

Figure 2-68: Temperature dependent CTE

With air flow velocity given in Table 2-23, the temperature distribution is shown in Figure 2-69 at various times. 

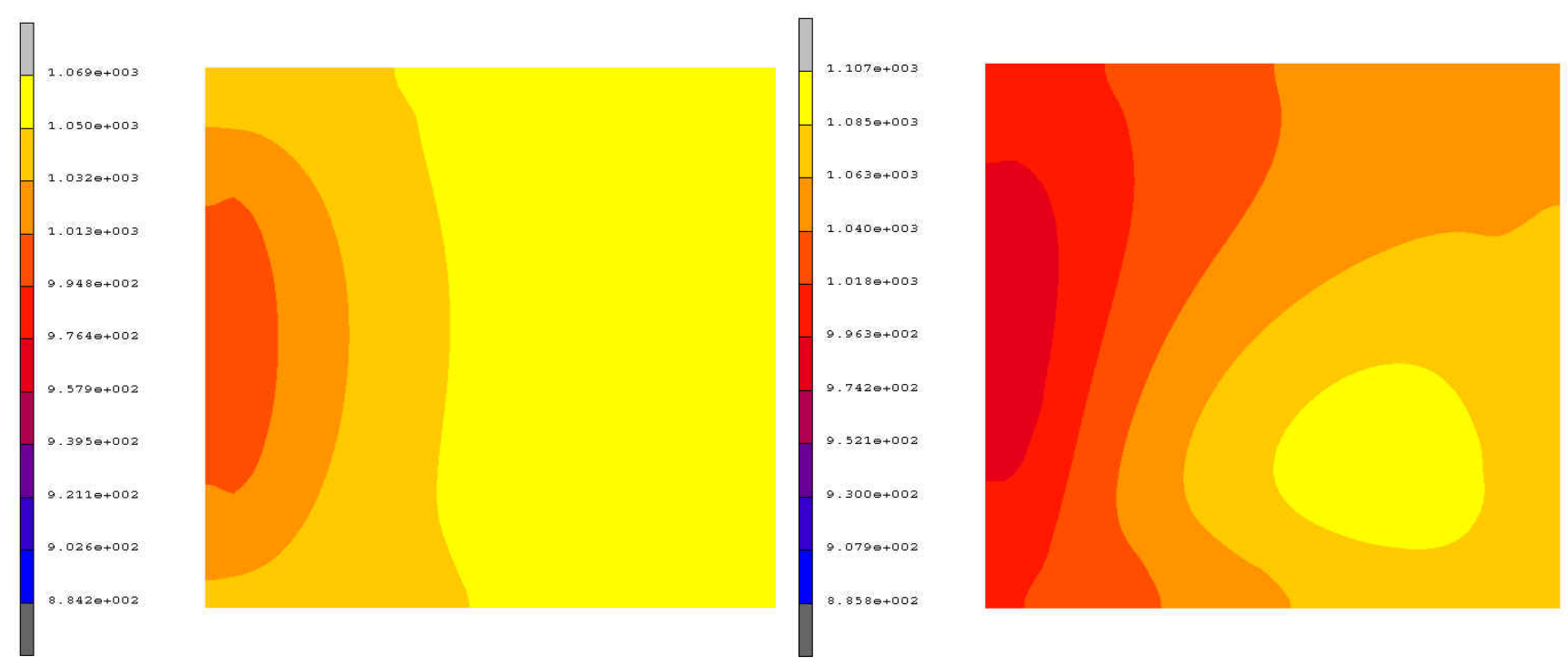

$$
\mathrm{t}=5.5 \mathrm{~min}
$$
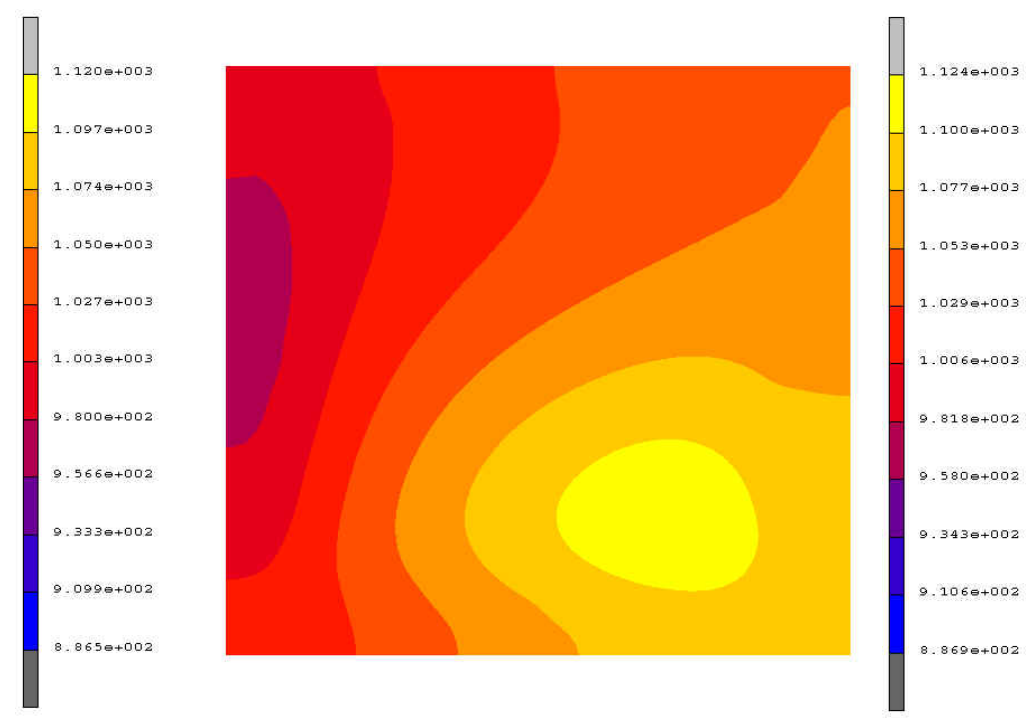

$\mathrm{t}=16.5 \mathrm{~min}$

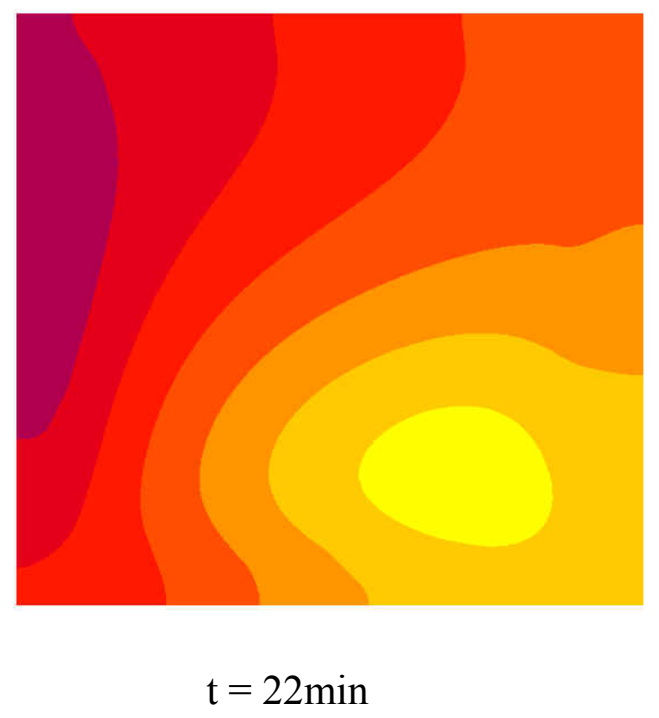



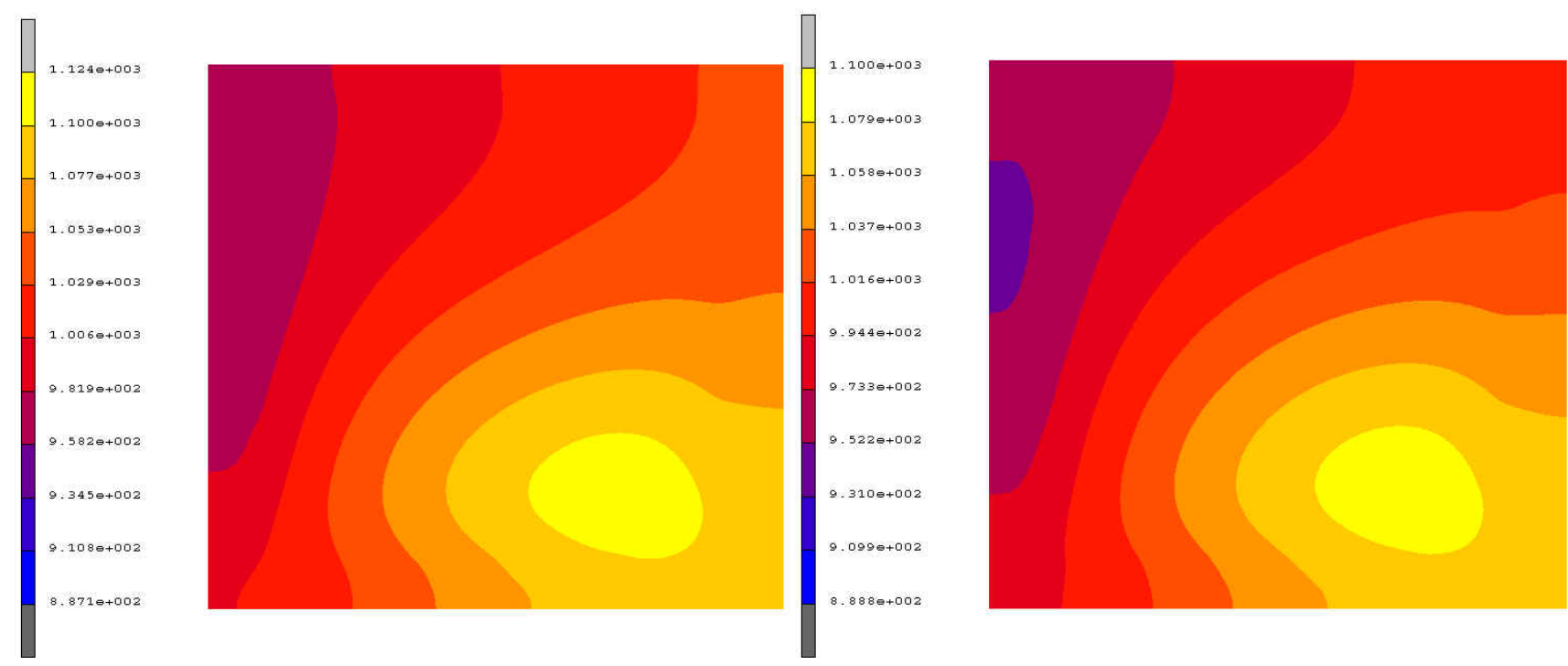

$\mathrm{t}=27.5 \mathrm{~min}$

$\mathrm{t}=160 \mathrm{~min}$

Figure 2-69: Temperature distribution at given air flow velocity $0.0169 \mathrm{~m} / \mathrm{s}$

The corresponding stress is obtained by means of thermal stress analysis with the temperature field from the heat transfer analysis by MARC EC software. The maximum Von Mises stress and maximum principal stress are shown in Figure 2-70 with respect to time. The maximum Von Mises stress and maximum principal stress does not occur at the steady state, but at the initial stage, and the maximum Von Mises stress and maximum principal stress are about twice of that at the steady state. It means that the initial state during the cool down process is much more dangerous than the steady state. Because the temperature of inlet air flow is lower than the initial temperature of the cell/stack, the air flow with the lower temperature will lead to a large temperature gradient at the initial state of the cool down. With the flow of the air in the channel, the temperature gradient will decrease and reach the steady state. The Figure 2-71 shows the effect of air flow velocity on the maximum von Mises stress. The dependency of stress on the flow velocity is not monotonic. Shown in Figure 2-72 is the maximum stress vs. the air flow velocity. It may is seen that the maximum stress occurs when the air velocity is about $5.5 \mathrm{~m} / \mathrm{s}$. Figure 2-73 shows us the maximum von Mises stress at various velocities at various times. This shows us that it will be necessary to optimize the flow velocity and other factors in order to shorten the start-up/cool down time without damaging the cell. 


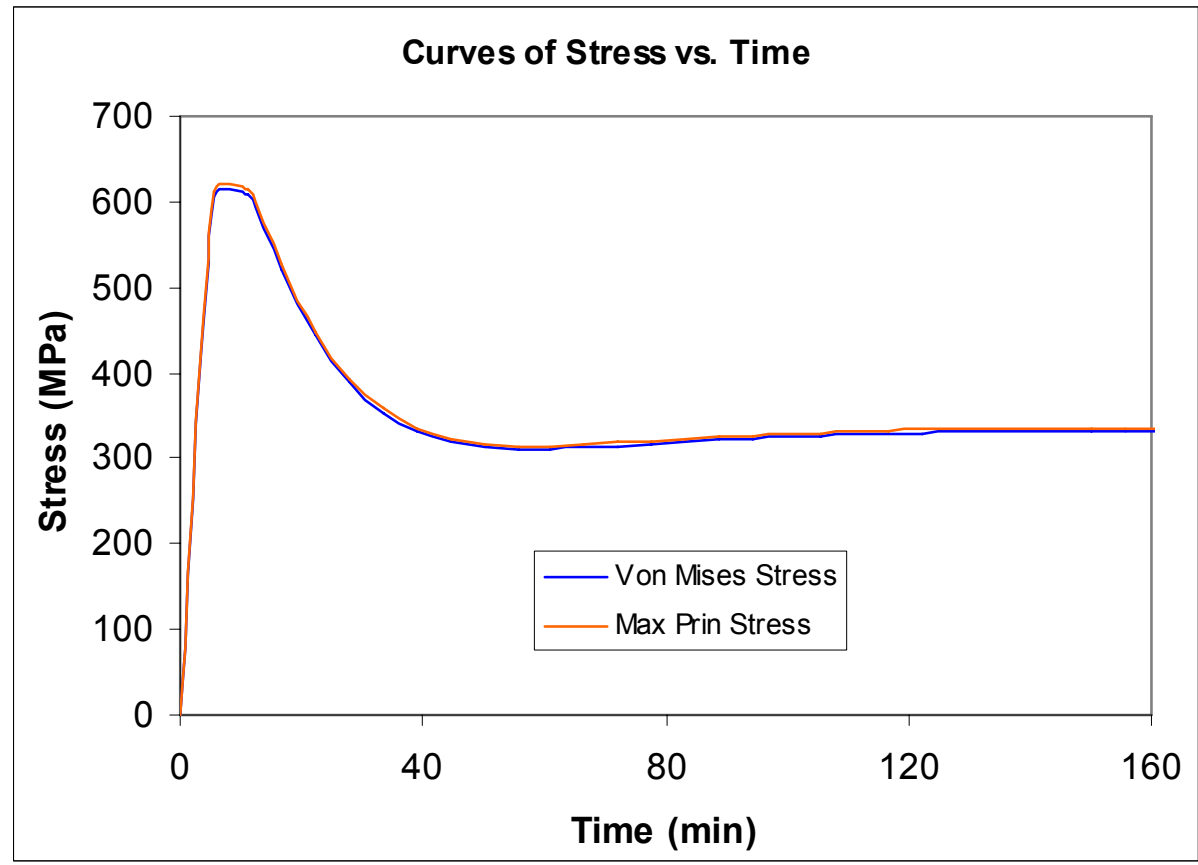

Figure 2-70: Curves of Von Mises stress and maximum principal stress with respect to time

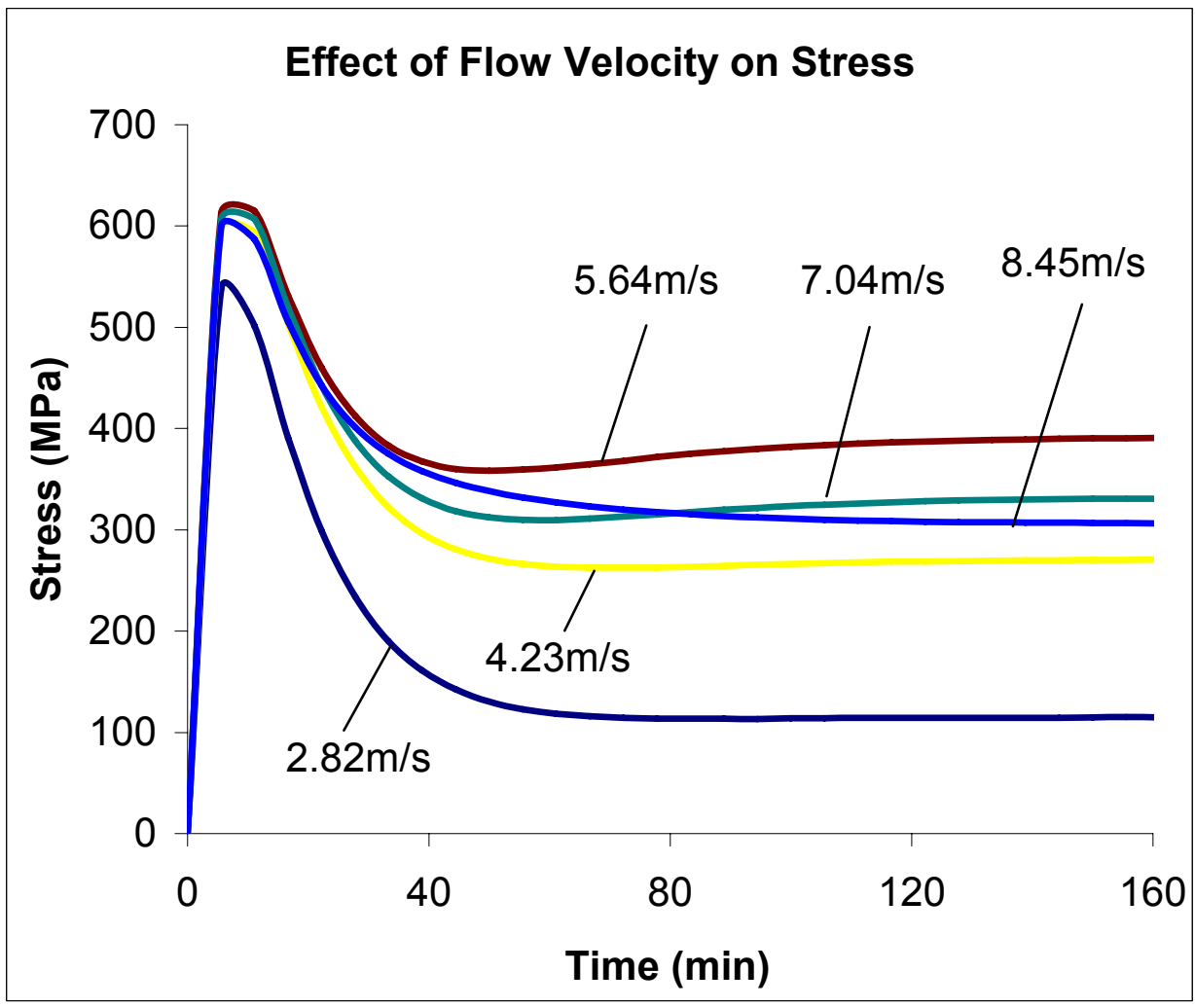

Figure 2-71: Effect of air flow velocity on stress 


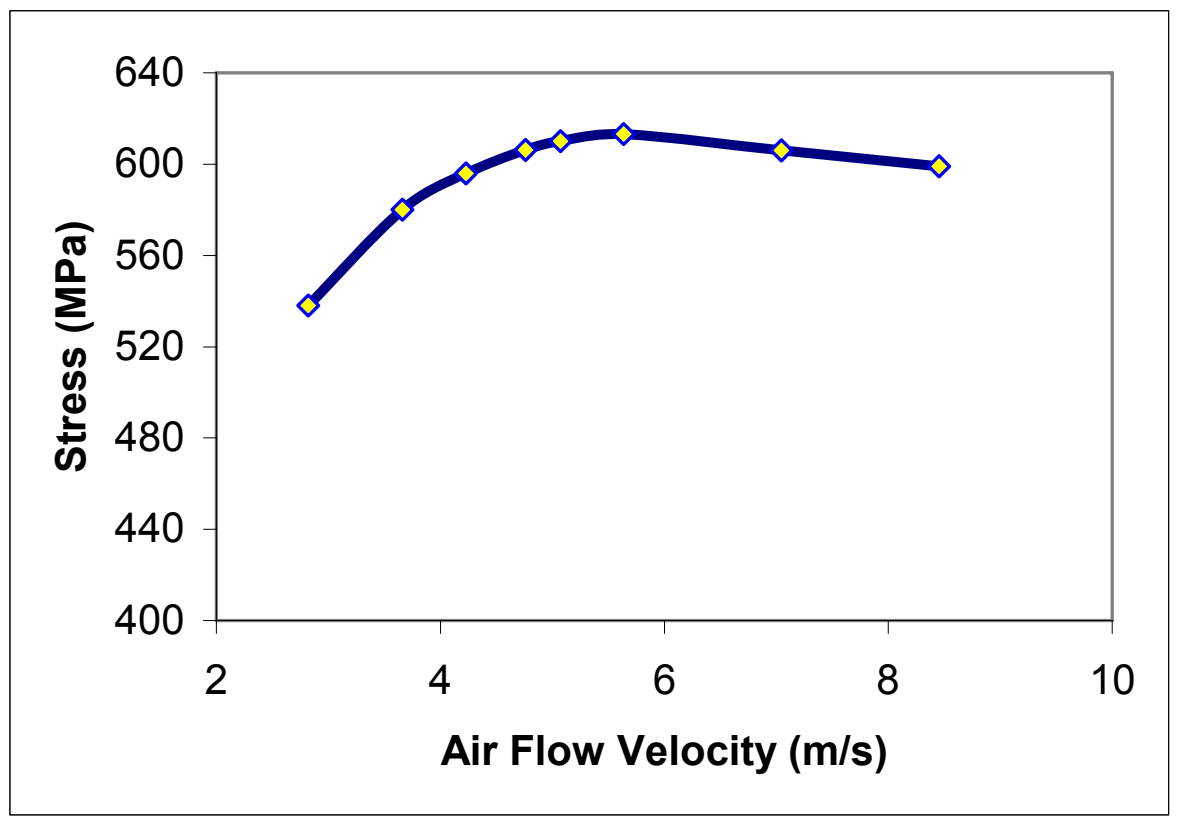

Figure 2-72: Curve of largest maximum stress over air flow velocity at infinite cooling rate

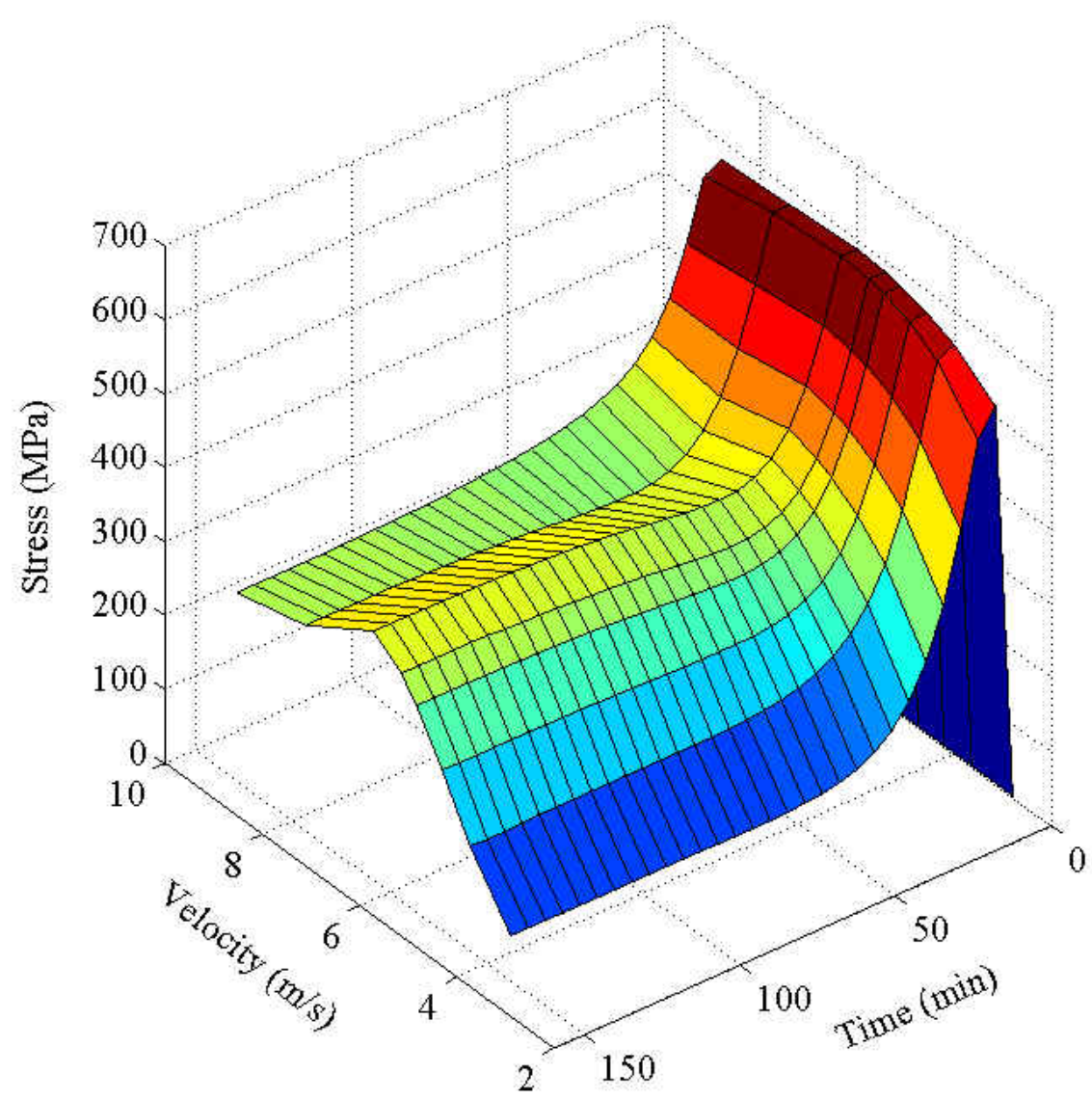

Figure 2-73: Maximum Von Mises stress at various velocities at various times 
Comparison of Figure 2-66 and Figure 2-73 indicates that both the analytical model and the FEM model show similar trend, although the magnitude of the stress and the velocity at which the maximum stress occurs are different. These differences are due to the simplifications used in the analytical model.

\section{Thermal Modeling}

\subsection{Importance of Thermal Radiation Heat Transfer}

As an initial approximation, the radiative heat flux within the cathode, electrolyte and anode was evaluated based on a diffusion model (Rosseland radiative model) valid for optically thick materials. The optical properties of Yttrium- Stabilized Zirconium (YSZ) and $\mathrm{Sr}$ doped $\mathrm{LaMnO}_{3}$ (LSM) were obtained from literature and employed in the Fluent model. Preliminary calculations show a drop of $\sim 50 \mathrm{~K}$ in the maximum temperature with the inclusion of radiation effects, see Figure 3-1. Moreover, inclusion of radiation results in a uniform distribution of the temperature field within the fuel cell due to higher effective thermal conductivity of the cathode and anode layers.

Unfortunately, the diffusion model is not applicable in the cathode and anode regions where the calculated optical properties indicate their failure to meet the optically thick material criteria. Currently, alternate radiation models, namely the "SchusterSchwarzschild" two-flux approximation and the Discrete Ordinate Method, are being evaluated. User Defined Functions (UDFs) need to be written in order to implement the "Schuster-Schwarzschild" two-flux radiation model in the fuel cell calculations, as Fluent does not have any in-built capabilities.

Familiarity with the Fluent code was achieved. Subsequently, radiative diffusion model calculations were carried out using the solver. Optical properties of the fuel cell material were used as inputs in the radiation model. Limitations with the present model were identified and future research plans were formulated.

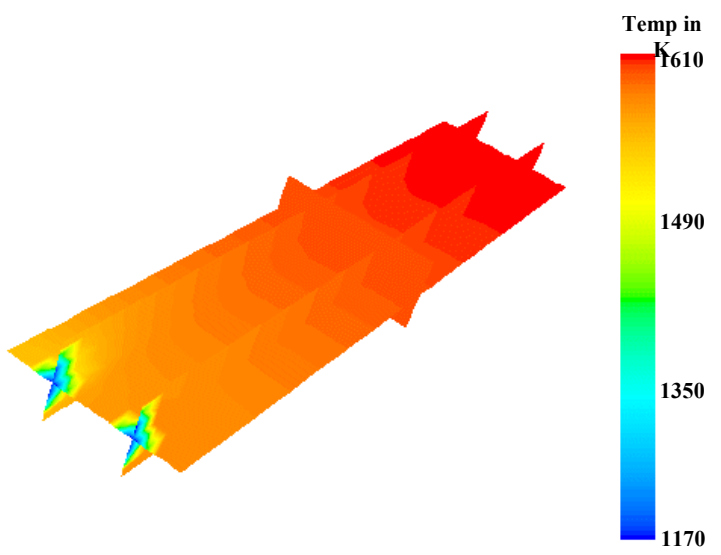

(a) without radiation

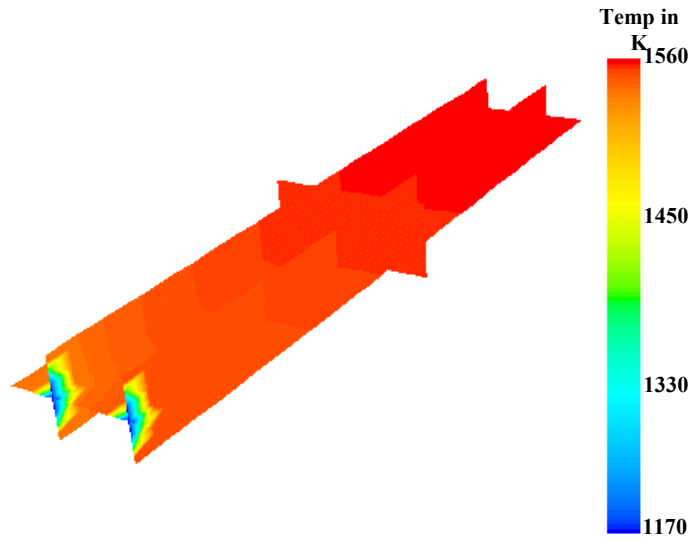

(b) with radiation

Figure 3-1: Temperature contours along (i) mid-oxidant-flow plane, (ii) mid-fuel-flow plane, (iii) mid-plane of fuel cell for Fluent runs with and without the radiative diffusion 
model. Inclusion of radiation effects result in more uniform distribution of the temperature field in the fuel cell.

\subsection{Two-flux Approximation for Numerical Calculation of Radiative Heat Transfer}

Figure 3-2 shows the temperature variation along lines passing through the center of the cathode-electrolyte and the anode-electrolyte walls, for the cases with and without inclusion of radiation effects. The radiation heat transfer is modeled using the Discrete Ordinates (DO) method with the representative optical properties of the electrodes and electrolyte obtained from the literature. As a first step, radiation in the solid electrolyte region is neglected. As expected, a drop in temperature is observed with the inclusion of radiation heat transfer in the electrodes of the fuel cell.

The inclusion of radiation effects in the solid electrolyte region is not entirely straightforward and is further complicated
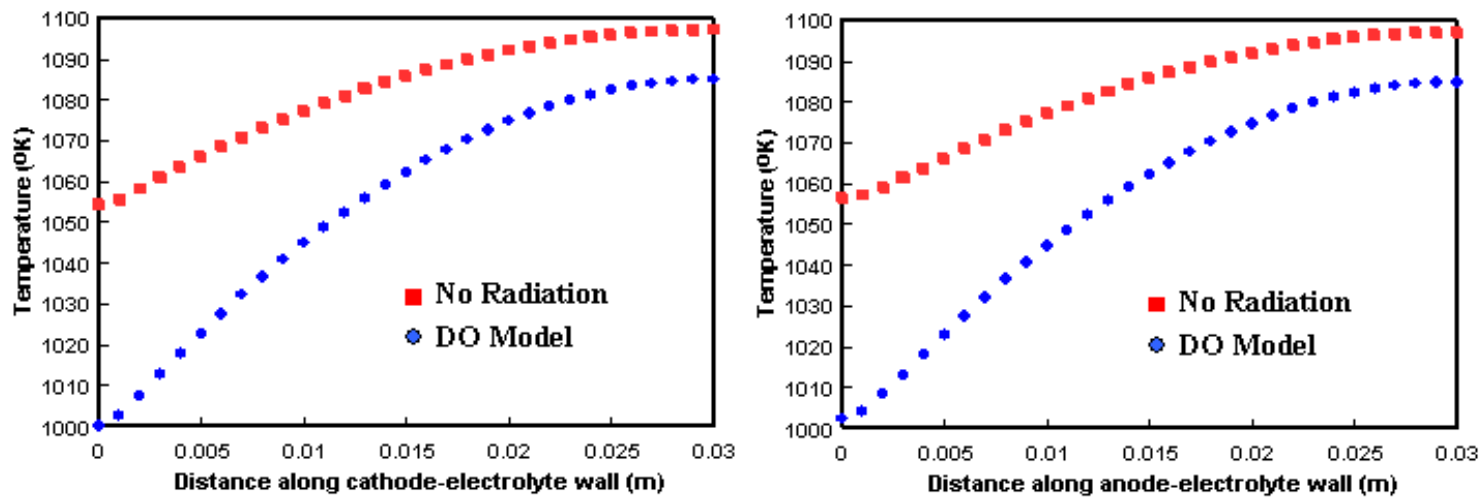

Figure 3-2: Temperature comparison for cases with and without radiation effects.

by the presence of optically thick electrodes. The high absorption coefficient values of the electrodes, coupled with the optical properties at the electrolyte interface walls result in poor convergence.

The problem with convergence using the DO method can be overcome by employing the Schuster-Schwartzchild two-flux approximation, detailed in the Appendix D, for treating thermal radiation transport in the optically thin YSZ electrolyte, with the Rosseland radiative thermal conductivity model accounting for radiation effects in the optically thick Ni-YSZ and LSM electrodes. The thermal radiation heat transfer is coupled to the overall energy conservation equations through the divergence of the local radiative flux.

Numerical experiments are currently being carried out to study the effectiveness of the two-flux modeling scheme in capturing the radiation heat transfer process in the fuel cell. Simultaneously, efforts are also on to address the convergence issues faced on inclusion of radiation in the solid electrolyte using the DO model.

\subsubsection{Porous Media Modeling:}

The currently implemented models for the fluid flow, species transport, and heat transfer in the porous electrodes have been deduced, although only based on the FLUENT source code developed by the NETL team. A preliminary literature survey was 
carried out to identify the modification of existing models, and to characterize the parameters that need to be accounted in the energy, momentum and species conservation equations for a an improved modeling of the physical processes taking place in the porous electrodes.

Specifically, the current FLUENT model uses the simplest and least accurate Darcy's model for simulation of the gas flow in the electrodes. It would be desirable to implement the Forchheimer-Brinkman extended D'Arcy model for momentum conservation which is given by

$$
\frac{1}{\varepsilon^{2}} \nabla \cdot(\rho \vec{V} \vec{V})=-\nabla p+\nabla \cdot\left(\mu_{e} \nabla \vec{V}\right)-\frac{\mu \vec{V}}{K}-\rho f \frac{|\vec{V}| \vec{V}}{\sqrt{K}}+\rho \vec{g} \beta\left(T-T_{\infty}\right)
$$

where $\rho, \mu, \mu_{e}, K, f$, and $\varepsilon$ are the fluid density, dynamic viscosity, effective viscosity, permeability, intertia coefficient, and porosity, respectively. The specific models for the latter four parameters will have to be developed for the porous materials used in the SOFC. The last term in equation (3.1) is the representation of the buoyancy driven natural convection effects through the Boussinesq-Oberbeck approximation. The natural convection flow may be very significant in the fuel cells owing to significant local thermal gradients within the electrodes, and thus need to be accounted for an accurate description of the local velocity field within the fuel cell electrodes.

The species transport is governed by the following mass conservation equation:

$$
\nabla \cdot\left(\rho \vec{V} Y_{i}\right)=\nabla \cdot\left(D_{i} \nabla Y_{i}\right)+S_{i}
$$

where $D_{i}$ and $S_{i}$ refer to the effective diffusion coefficient and production/depletion rate of species $i$ respectively. While $S_{i}$ is to be modeled through electrochemistry of relevant reactions, $D_{i}$ has to be obtained by accounting for molecular diffusion, Knudsen diffusion, dispersion, and thermal diffusion effects. Because of the small diameter of the electrode pores, the Knudsen diffusion may be very significant as it specifies the resistance to the transport of a component due to ballistic molecule-pore walls collisions and needs to be included in the diffusion modeling of the porous electrodes. Meanwhile, the tendency of a component to diffuse under the influence of the temperature gradients across the electrodes in the fuel cell, known as the Soret effect, can be captured through modeling the thermal diffusion effects. Finally, the species dispersion due to local gradients in the flow velocity results in further enhancement of the mass transfer and can be accounted for by using, for example, the Taylor dispersion model.

Heat transfer through porous media in Fluent is represented based on the assumption of thermal equilibrium between the solid structure and the gas flowing through it. The assumption of the local thermal equilibrium is only justified when

a) there is no significant heat generation in the porous media

b) the difference between the solid and fluid thermal conductivities is negligible

c) the Reynolds number or the flow velocities through the porous media is high 
Unfortunately, none of the above conditions are satisfied in the fuel cell electrodes thereby requiring the energy conservation to be carried out through generalized nonequilibrium thermal model:

$$
\begin{aligned}
& \nabla \cdot\left(\rho \vec{V} c_{p} T_{g}\right)=\nabla \cdot\left(k_{g, \text { eff }} \nabla T_{g}\right)-h_{v}\left(T_{g}-T_{s}\right)+\sum S_{i} \Delta H_{i} \quad \text { (Gas phase) } \\
& 0=\nabla \cdot\left(k_{s, \text { eff }} \nabla T_{s}\right)+h_{v}\left(T_{g}-T_{s}\right) \quad(\text { Solid phase })
\end{aligned}
$$

where $k_{g, e f f}, k_{s, e f f}, h_{v}$ and $\Delta H_{i}$ are the effective gas-phase thermal conductivity, effective solid-phase thermal conductivity, the local volumetric convective heat transfer coefficient and the enthalpy of reaction species, respectively. The models for the first three effective heat transport properties will have to be developed based on the expected flow regimes and the specific morphology of the porous electrodes. As in the case of species transport, he model for the effective gas-phase thermal conductivity will have to include the contributions due to molecular heat transport and Knudsen ballistic heat transport as well as the dispersion and diffusion-thermo (Dufort) effects.

\subsection{Engineering Code to Compare Performance and Reliability Trends.}

Figure 3-3 and Figure 3-4 show the temperature variation along lines passing through the center of the cathode-electrolyte and the anode-electrolyte bi-layers, respectively, for the cases with and without inclusion of radiation effects. The radiation transfer is treated using the discrete ordinate method (the most accurate but computationally very expensive) as well as using the simplified two-flux approximation which is applicable for the optically thin electrolyte of SOFC. Radiation transfer in optically thick electrodes is simulated using Schuster-Shwarzchild's diffusion approximation, which is detailed in the previous reports. The representative optical properties of the electrodes and electrolyte are those obtained from the literature. As expected, inclusion of radiative transfer leads to a significant drop in the temperature level (by as much as $150^{\circ} \mathrm{C}$ ) which is accompanied by an increase in the cell voltage by almost $20 \%$.

Further, we developed a computationally efficient sub-module for solving a radiative transfer problem using the two-flux approximation. The module was integrated into the overall NETL FLUENT code and validated by comparing to the results with those obtained using the most accurate,but computationally very expensive, discrete ordinate method. As it is indicated on Figure 3-3 and Figure 3-4, the maximum difference between the temperature predictions obtained using the most accurate discrete ordinate (DO) method and the approximate two-flux method we used is less than $10^{\circ} \mathrm{C}$. At the same time, use of the two-flux approximation resulted in tremendous computational savings (by one order of magnitude) or reduction in CPU time from 658 minutes (DO method, FLUENT implementation) to just 76 minutes (two-flux approximation). The results of these calculations are supported by the previous independent study of Ceramatec ( $\mathrm{J}$. Hartvigsen, S. Elangovan, and A. Khandkar, ZIRCONIA V paper number 105). 


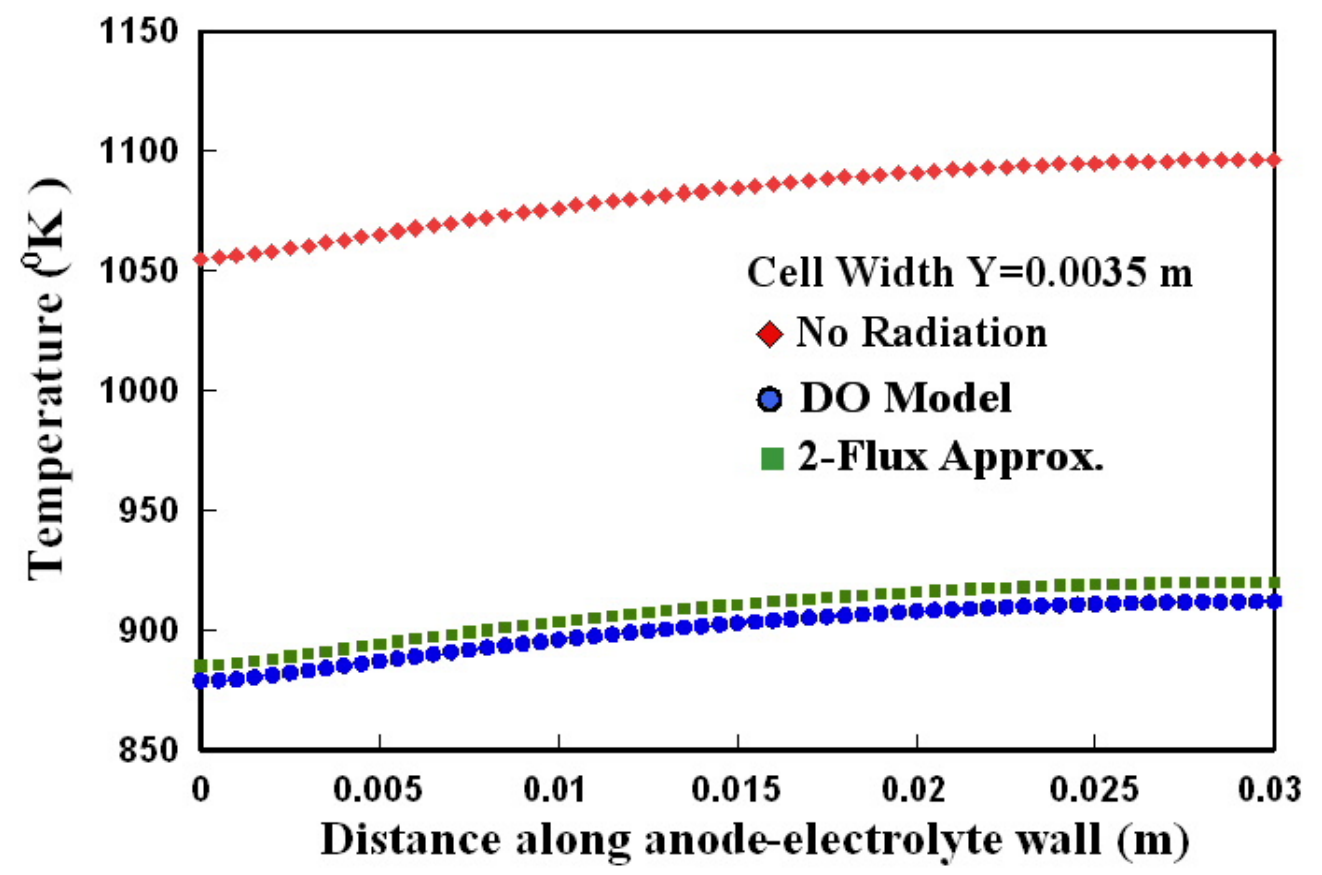

Figure 3-3: Effect of radiative transfer on the SOFC temperature (temperature along the anode-electrolyte interface)

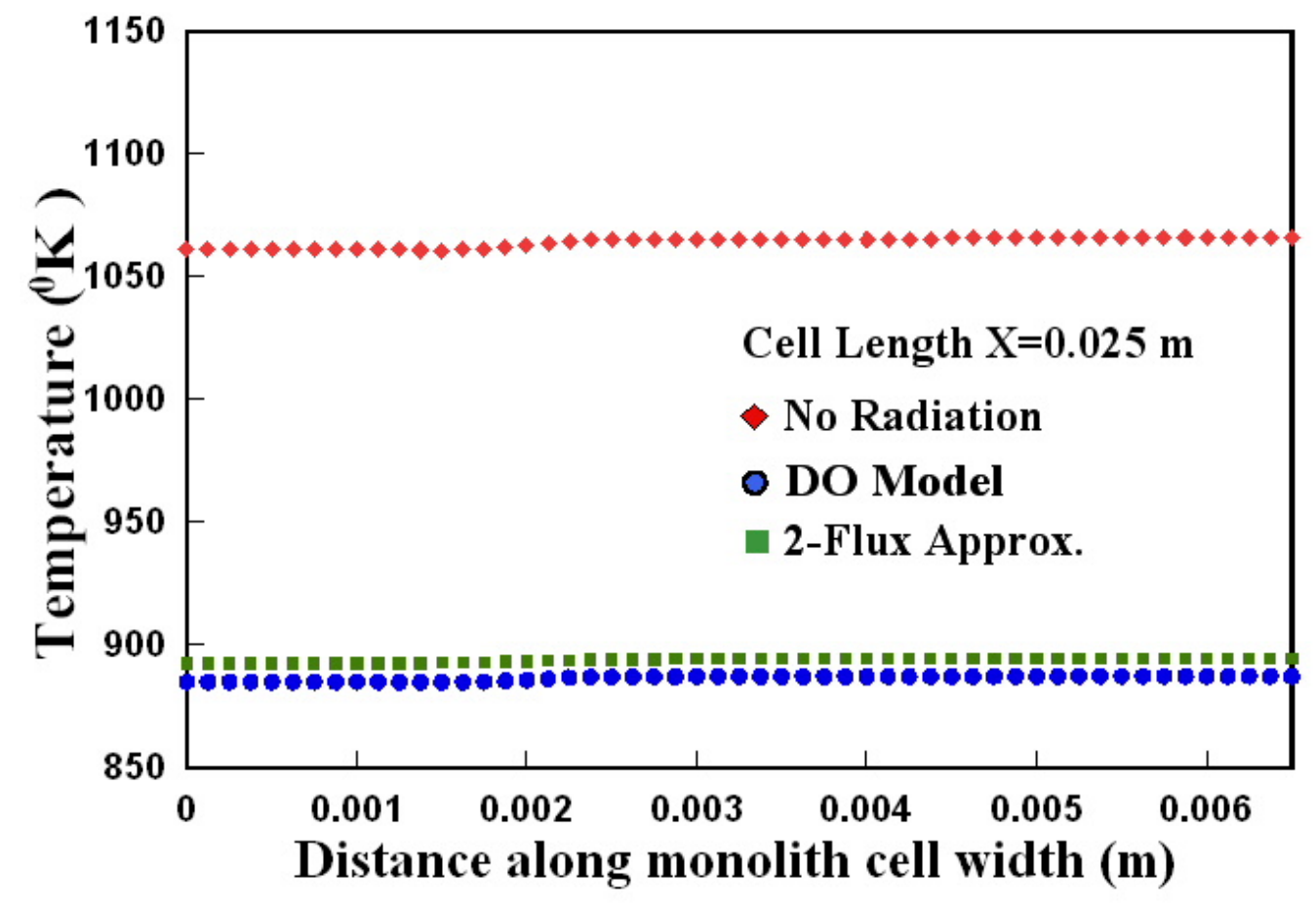

Figure 3-4: Effect of radiative transfer on the SOFC temperature (temperature along the cathode-electrolyte interface)

Numerical experiments are currently being carried out to study the effectiveness of the two-flux modeling scheme in capturing the radiation heat transfer process in the fuel 
cell. Simultaneously, efforts are also underway to address the convergence issues faced on inclusion of radiation in the solid electrolyte using the DO model.

Porous Media Mass Transport Modeling:

The species transport is governed by the following mass conservation equation:

$$
\nabla \cdot\left(\rho \vec{V} Y_{i}\right)=\nabla \cdot \rho\left(D_{i} \nabla Y_{i}-D_{i, T} \frac{\nabla T}{T}\right) \pm S_{i}
$$

where $D_{i}$ and $S_{i}$ refer to the effective diffusion coefficient and production/depletion rate of species $i$, respectively. While $S_{i}$ is to be modeled through the chemistry/electrochemistry of relevant reactions, $D_{i}$ has to be obtained by accounting for molecular diffusion, Knudsen diffusion, dispersion, and thermal diffusion effects.

$$
D_{i}=\frac{\varepsilon}{\tau}\left(\frac{1-\alpha_{i} x_{i}}{D_{i, m}}+\frac{1}{D_{i, K}}+\frac{1}{D_{i, d}}\right)^{-1}
$$

Here, $\varepsilon$ is the porosity, $\tau$ is tortuosity, $x_{i}$ is the mole fraction of i-th component, and $\alpha_{i}=1-\left(\frac{M_{i}}{M_{m}}\right)^{1 / 2}$ is the factor depending on the molar weights of the mixture components.

The molecular diffusion coefficient of the mixture can be expressed in terms of the pair-wise binary diffusion coefficients $\left(D_{i k}\right)$ for the mixture components:

$$
D_{i, m}=\frac{1-x_{i}}{\sum_{k \neq i} \frac{x_{k}}{D_{i k}}}
$$

Because of the small diameter of the electrode pores, the Knudsen diffusion may be very significant as it specifies the resistance to the transport of a component due to ballistic molecule-pore walls collisions and needs to be included in the diffusion modeling of the porous electrodes. The Knudsen diffusion coefficient for i-th component of the mixture is expressed in terms of the mean pore radius ( $r$ ), the universal gas constant $(\mathrm{R})$, and the local absolute temperature $(\mathrm{T})$ as follows:

$$
D_{i, K}=\frac{2}{3}\left(\frac{8 R T}{\pi M_{i}}\right)^{1 / 2} r
$$

Further, the species dispersion due to local gradients in the flow velocity results in further enhancement of the mass transfer and can be accounted for by using, for example, the Taylor dispersion model in which the dispersion coefficient is expressed as a function of the local flow Peclet number and porosity $\left(D_{i, d}=f(P e, \varepsilon)\right)$.

Finally, the last term in the brackets accounts for the tendency of a component to diffuse under the influence of the temperature gradients across the electrodes in the fuel cell, known as the Soret effect. It can be captured through modeling the thermal diffusion effects. The thermal diffusion coefficient is expressed in terms of the local temperature 
$(\mathrm{T})$, the molar weights $\left(\mathrm{M}_{\mathrm{i}}\right)$, molar fractions $\left(\mathrm{x}_{\mathrm{i}}\right)$, and mass fractions $\left(\mathrm{y}_{\mathrm{i}}\right)$ of the mixture components.

$$
D_{i, T}=-2.59 \times 10^{-7} T^{0.659}\left[\frac{M_{i}^{0.511} x_{i}}{\sum_{i=1}^{N} M_{i}^{0.511} x_{i}}-y_{i}\right] \bullet\left[\frac{\sum_{i=1}^{N} M_{i}^{0.511} x_{i}}{\sum_{i=1}^{N} M_{i}^{0.489} x_{i}}\right]
$$

Our on-going efforts are focused on quantifying the relative importance of all of the above-mentioned effects on mass transport of reagents within the electrodes of the SOFC in order to identify the rate-limiting step under the various operating conditions and electrode designs.

\subsection{Theoretical Methodology for Modeling Gas flow, Mass, and Heat transfer}

The species transport is governed by the following mass conservation equation:

$$
\nabla \cdot\left(\rho \vec{V} Y_{i}\right)=\nabla \cdot \rho\left(D_{i} \nabla Y_{i}-D_{i, T} \frac{\nabla T}{T}\right) \pm S_{i}
$$

where $D_{i, T}$ is the thermal diffusion coefficient, $D_{i}$ is the mass diffusion coefficient and $S_{i}$ production/depletion rate of species $i$. While $S_{i}$ is to be modeled through electrochemistry of relevant reactions, $D_{i}$ has to be obtained by accounting for molecular diffusion, Knudsen diffusion, dispersion, and thermal diffusion effects. Using the parallel pore model, the effective gas diffusion coefficient for component $i$ in the porous media can be expressed as,

$$
D_{i}=\frac{\varepsilon}{\tau}\left(\frac{1-\alpha_{i} x_{i}}{D_{i, m}}+\frac{1}{D_{i, K}}+\frac{1}{D_{i, d}}\right)^{-1}
$$

Here, $\varepsilon$ is the porosity, $\tau$ is tortuosity, $x_{i}$ is the mole fraction of $i$-th component, and $\alpha_{i}=1-\left(\frac{M_{i}}{M_{m}}\right)^{1 / 2}$ is the factor depending on the molar weights of the mixture components.

\subsubsection{Binary Mass Diffusion:}

In a multicomponent gas system, the molecular diffusion coefficient of the component $i$ is expressed in terms of the pair-wise binary diffusion coefficients $\left(D_{i k}\right)$ by:

$$
D_{i, m}=\frac{1-x_{i}}{\sum_{k \neq i} \frac{x_{k}}{D_{i k}}}
$$

The current empirical formulation for the binary diffusion coefficient, as defined in [Bird, R.B., Stewart, W.E., and Lightfoot, E.N., Transport Phenomena, John Wiley and Sons, 
New York, 1960], is replaced with a first order approximation of the Kinetic theory, and is given by [Reid, R. C. and Prausnitz, J M., The Properties of Gases and Liquids, McGraw-Hill, New York, 1977]:

$$
D_{i j}=1.8809 \times 10^{-7} \frac{\sqrt{T^{3}\left(\frac{1}{M_{i}}+\frac{1}{M_{j}}\right)}}{p \Omega_{D} \sigma_{i j}}
$$

where $T$ is the temperature $(\mathrm{K}), p$ is the pressure $(\operatorname{atm}), \sigma_{i j}$ is a characteristic length $\left(\mathrm{A}^{\circ}\right)$ and $\Omega_{D}$ is the collision integral for diffusion. Using the Lennard-Jones 12-6 potential model, $\Omega_{D}$ is given by:

$$
\Omega_{D}=\frac{A}{T^{* B}}+\frac{C}{e^{D T^{*}}}+\frac{E}{e^{F T^{*}}}+\frac{G}{e^{H T^{*}}}
$$

where the constants $A-H$ are, $A=1.06036, B=0.15610, C=0.19300, D=0.47635, E=$ $1.03587, F=1.52996, G=1.76474, H=3.89411$, and $T^{*}$ is defined by:

$$
T^{*}=\frac{k T}{\varepsilon_{i j}}
$$

The characteristic Lennard-Jones energy $\left(\varepsilon_{i j}\right)$ and length $\left(\sigma_{i j}\right)$ are given by:

$$
\begin{aligned}
& \sigma_{i j}=\frac{\sigma_{i}+\sigma_{j}}{2} \\
& \varepsilon_{i j}=\left(\varepsilon_{i} \varepsilon_{j}\right)^{1 / 2}
\end{aligned}
$$

The parameters used for simulation are given in Table 1 [Hirschfelder, J. O., Curtiss, C. F., and Bird, R. B., Molecular Theory of Gases and Liquids, John Wiley and Sons, Inc., New York, 1954].

Figure 3-5 plots the variation in mass fraction of $\mathrm{H}_{2}$ along the centerline of the fuelflow channel, predicted using the binary mass diffusion coefficients calculated through the Kinetic theory and compares with the results obtained from existing model in the NETL Fluent code. Significant changes in the diffusion of $\mathrm{H}_{2}$ at the entry region of the fuel-flow channel are observed on the implementation of the Kinetic theory.

Table 3-1: List of characteristic Lennard-Jones length and energy [Hirschfelder, J. O., Curtiss, C. F., and Bird, R. B., "Molecular Theory of Gases and Liquids", John Wiley and Sons, Inc., New York, 1954.]

\begin{tabular}{|c|c|c|c|c|c|c|}
\hline Species & $\mathrm{N}_{2}$ & $\mathrm{O}_{2}$ & $\mathrm{H}_{2}$ & $\mathrm{H}_{2} \mathrm{O}$ & $\mathrm{CO}_{2}$ & $\mathrm{CO}$ \\
\hline$\sigma_{i}\left(\mathrm{~A}^{\circ}\right)$ & 3.681 & 3.433 & 2959 & 2.641 & 3.996 & 3.59 \\
\hline$\varepsilon_{i}(\mathrm{~K})$ & 91.5 & 113 & 36.7 & 809.1 & 190 & 110 \\
\hline
\end{tabular}




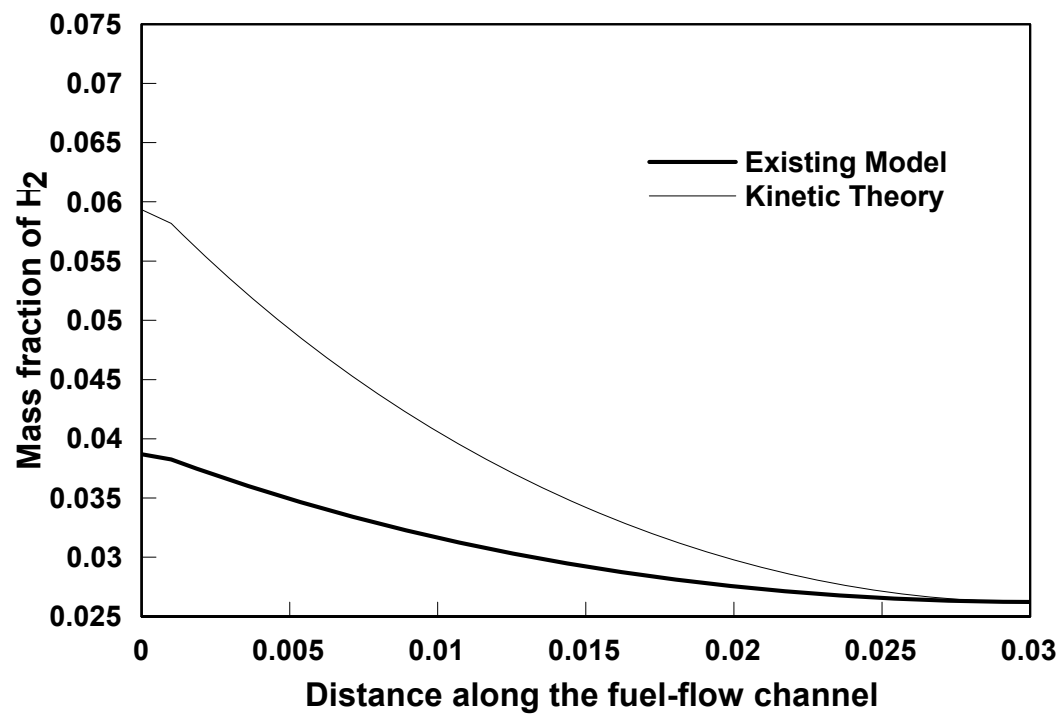

Figure 3-5: Comparison of variation in mass fraction of $\mathrm{H}_{2}$ along the centerline of fuel-flow channel.

\subsubsection{Knudsen Diffusion:}

The Knudsen number, given by:

$$
K n=\frac{\lambda(\text { mean free path of gas molecules })}{C \text { (average pore size })}
$$

is a measure of resistance to the transport of a component due to ballistic molecule-pore walls collisions. The Knudsen diffusion coefficient, expressed in terms of the mean pore radius $(r)$, the universal gas constant $(R)$, and the local temperature $(T)$ as:

$$
D_{i, k}=\frac{2}{3}\left(\frac{8 R T}{\pi M_{i}}\right)^{1 / 2} r
$$

becomes important for Knudsen numbers larger than 10. The mean free path of $\mathrm{H}_{2}$ at $1000{ }^{\circ} \mathrm{K}$ and atmospheric pressure is $0.23 \mu \mathrm{m}$. An average pore size of $2.6 \mu \mathrm{m}$ for the anode substrate results in a Knudsen number of 0.08. Table 3-2 shows the percentage change in cell output voltage with the inclusion of Knudsen diffusion model when compared to mass transport without the model. As expected, the Knudsen diffusion effect is negligible for the size ranges considered.

Table 3-2: Variation of cell output voltage with average pore size due to Knudsen diffusion.

\begin{tabular}{|c|c|c|}
\hline Size $(\boldsymbol{\mu m})$ & Cell Voltage $(\mathbf{V})$ & \% Change \\
\hline 0.26 & 0.6724 & 1.800 \\
\hline 2.60 & 0.6621 & 0.240 \\
\hline 26.0 & 0.6606 & 0.015 \\
\hline
\end{tabular}




\subsubsection{Dispersion Effect:}

The species dispersion due to local gradients in the flow velocity results in further enhancement of the mass transfer and can be accounted for by using, for example, the Taylor dispersion model in which the dispersion coefficient is expressed as a function of the local flow Peclet number and porosity $\left(D_{i, d}=f(P e, \varepsilon)\right)$. Peclet number, defined in terms of liquid velocity $(u)$ and radius of pore diameter $(R)$ as:

$$
P e=\frac{u R}{D_{i, m}}
$$

is a measure of the mass dispersion effect caused due to flow when compared to molecular diffusion process. It is expected that for low Peclet numbers $(<1)$, dispersion effects do not contribute significantly to the total diffusivity as molecular diffusion dominates. Table 3-3 lists the Peclet number for $\mathrm{H}_{2}, \mathrm{O}_{2}$ and $\mathrm{H}_{2} \mathrm{O}$ in the anode and cathode regions based on the maximum velocity $\mathrm{u}$ and an average pore radius diameter of $60 \mu \mathrm{m}$. The low values of Peclet numbers suggest that the dispersion effect can be neglected without much loss in accuracy in mass transport of the species.

Table 3-3: Species Peclet number at anode and cathode regions based on maximum flow velocity

\begin{tabular}{|c|c|c|c|}
\hline Species & $\mathbf{H}_{\mathbf{2}}$ & $\mathbf{O}_{\mathbf{2}}$ & $\mathbf{H}_{\mathbf{2}} \mathbf{O}$ \\
\hline Peclet \# (Anode) & 0.012 & 0.041 & 0.034 \\
\hline Peclet \#(Cathode) & 0.048 & 0.170 & 0.138 \\
\hline
\end{tabular}

\subsubsection{Thermal Diffusion:}

The tendency of a component to diffuse under the influence of the temperature gradients across the electrodes in the fuel cell, known as the Soret effect, is captured through the thermal diffusion effects. Fluent incorporates an empirically based composition dependent thermal diffusion coefficient expressed in terms of the local temperature $(T)$, the molar weights $\left(M_{i}\right)$, molar fractions $\left(x_{i}\right)$, and mass fractions $\left(y_{i}\right)$ of the mixture components as:

$$
D_{i, T}=-2.59 \times 10^{-7} T^{0.659}\left[\frac{M_{i}^{0.511} x_{i}}{\sum_{i=1}^{N} M_{i}^{0.511} x_{i}}-Y_{i}\right] \bullet\left[\frac{\sum_{i=1}^{N} M_{i}^{0.511} x_{i}}{\sum_{i=1}^{N} M_{i}^{0.489} x_{i}}\right]
$$

The above form of Soret diffusion coefficient causes heavy molecules to diffuse less rapidly, and light molecules to diffuse more rapidly, towards heated surfaces. Figure 3-6 plots the temperature variation across the thickness of the fuel cell, at cross-sections near the fuel-air entry and exit regions. The lack of large temperature gradients across the electrodes results in negligible thermal diffusion effect. Implementation of Soret effect results in a $0.03 \%$ change in the cell output voltage. 


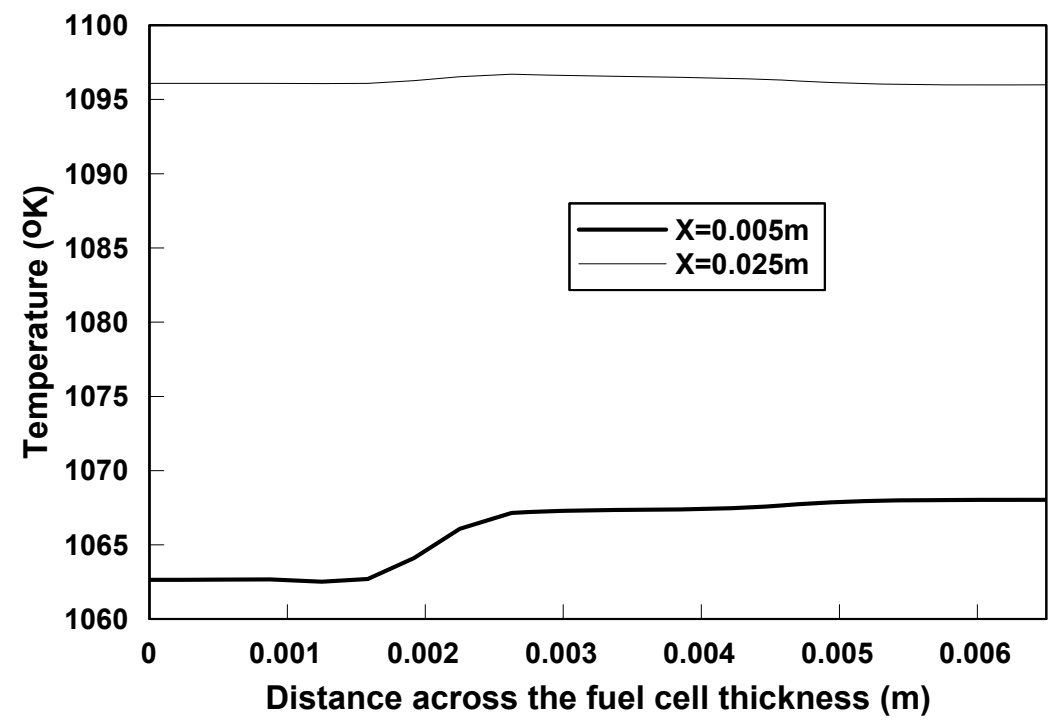

Figure 3-6: Variation of temperature across the fuel cell thickness

\subsubsection{Flow Transport Modeling}

The Forchheimer-Brinkman extended D'Arcy model for momentum conservation is given by:

$$
\frac{1}{\varepsilon^{2}} \nabla \cdot(\rho \vec{V} \vec{V})=-\nabla p+\nabla \cdot(\mu \nabla \vec{V})-\frac{\mu \vec{V}}{K}-\rho f \frac{|\vec{V}| \vec{V}}{\sqrt{K}}+\rho \vec{g} \beta\left(T-T_{\infty}\right)
$$

where $\rho, \mu, K, f$, and $\varepsilon$ are the fluid density, dynamic viscosity, permeability, inertia coefficient, and porosity, respectively. The permeability and inertia coefficient are tough to predict and usually modeled through semi-heuristic formulations. It is possible to replace the Darcy $\left(\frac{\mu \vec{V}}{K}\right)$ and the Inertia loss $\left(\rho f \frac{|\vec{V}| \vec{V}}{\sqrt{K}}\right)$ terms with a single source term in the momentum equations. Molerus and Schweinzer [Molerus, O. and Schweinzer, J.,

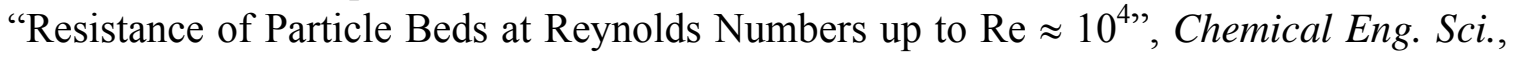
vol. 44, pp. 1071-1079, 1989] showed that the pressure drop for flows through a spherical particulate media with porosities in the range, $0.35 \leq \varepsilon \leq 0.64$, can be modeled through the momentum equation source term $\mathrm{S}$, given by:

$$
S=\frac{-18(1-\varepsilon) \rho U^{2}}{\operatorname{Re} \varepsilon^{2} d}\left\{1+0.692\left[A+0.5 A^{2}\right]+\frac{4}{\sqrt{\operatorname{Re}}}\left[1+0.12 A^{1.5}\right]+0.4+\frac{0.891 A}{\operatorname{Re}^{0.1}}\right\}
$$

where $A$ is a function of the porosity and Re refers to the Reynolds number of the flow. The above formulation is in the process of being implemented to study the effectiveness on the SOFC parameters.

\subsection{Summary of Radiative Properties Evaluation}

As previously reported the electrolyte material, $8 \mathrm{~mol} \%$ Yttria Stabilized Zirconia (8YSZ), is semitransparent in the near and mid-infrared spectra. It was also found that the 
extinction coefficient is a strong function of the wavelength of radiation. Thus, the twoflux approximation for optically thin media developed in Phase- 1 can still be used, but it has to be adapted to include the radiative heat transfer within this non-gray medium with spectrally varying optical properties.

Thinner samples of the anode and cathode materials were obtained from PNNL, and we measured their spectral reflectance and transmittance with FTIR spectrometer. The electrode (anode made of $40 \mathrm{vol} \% \mathrm{Ni} ; 60 \mathrm{vol} \%$ Y YSZ and cathode made of Sr-doped Lanthanum ferrite) samples appear to be opaque over the entire near and mid infrared spectra.

\subsubsection{Technical Description of the Experimental Work}

Figure 3-7 shows the data obtained from the FTIR measurements, namely the transmittance $(\operatorname{Tr})$ and reflectance $(R)$ obtained from $200 \mu \mathrm{m}$ thick samples of electrode materials. The surface reflectivity and absorption coefficient can be found in terms of reflectance and transmittance of the sample for the normal beam incidence from the following relations:

$$
\begin{aligned}
& R=\rho+\frac{\rho(1-\rho)^{2} e^{-2 \kappa L}}{1-\rho^{2} e^{-2 \kappa L}} \\
& \operatorname{Tr}=\frac{(1-\rho)^{2} e^{-\kappa L}}{1-\rho^{2} e^{-2 \kappa L}}
\end{aligned}
$$

where $\rho$ is the reflectivity, $\kappa$ is the absorption coefficient and $L$ is the thickness of the medium. The refractive index of the medium $(n)$ in turn can be obtained from the reflectivity through Fresnel's equation for an unpolarized light:

$$
\begin{aligned}
\rho & =\frac{\left(n-n_{\text {air }}\right)^{2}+k^{2}}{\left(n+n_{\text {air }}\right)^{2}+k^{2}} \\
k & =-\frac{1}{4} \frac{\ln (\kappa) \lambda}{\pi L}
\end{aligned}
$$

The measured transmittance shows that the samples are essentially opaque over the entire near and mid infrared spectra, and solution of equations 3.1 and 3.2 with values of $\operatorname{Tr}=0$ confirms infinitely large extinction coefficient over the entire IR spectrum of interest. This is because the electrodes are heavily doped with metals to increase their electronic conductivity, and the presence of free electrons in metals makes these electrode materials very efficient radiation absorbers over the entire spectrum. Further experiments could be performed with even thinner samples of electrode materials, however, it is not a high priority at this time because it is apparent that radiation heat transfer within the electrodes is fairly insignificant and only surface radiation must be accounted for. 


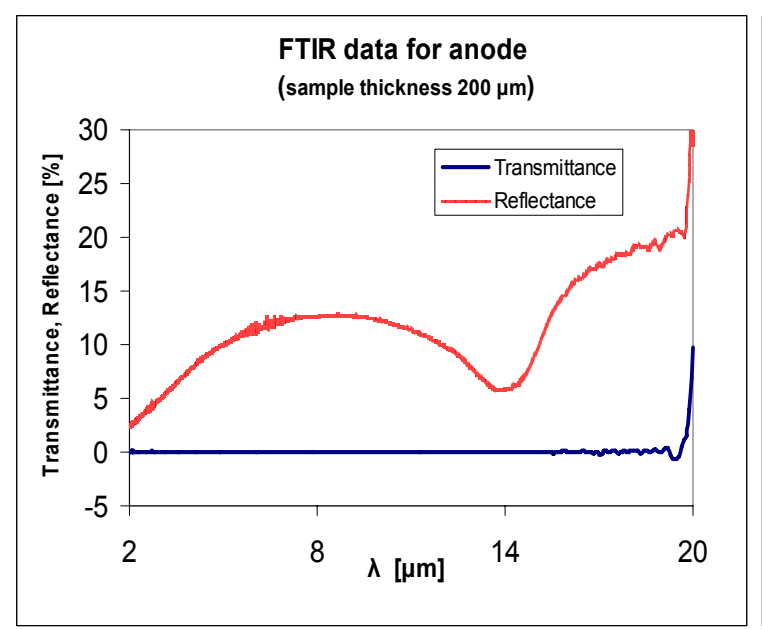

(a)

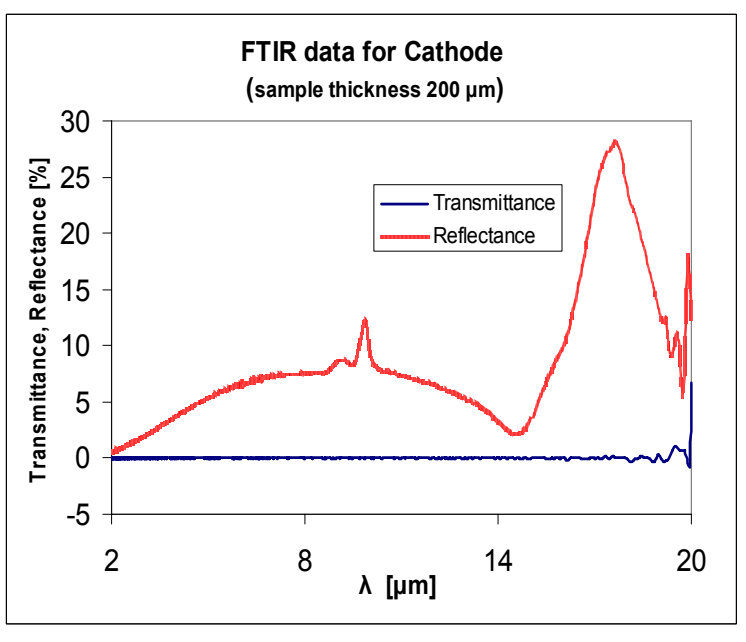

(b)

Figure 3-7: FTIR data for $200 \mu \mathrm{m}$ thick samples of anode (a) and cathode (b) material shows zero transmittance in the near and mid-infrared spectra.

\subsection{Code validation for solution of radiative heat flux}

The 2-flux formulation of spectral radiative heat flux in the optically thin yttriastabilized zirconia (YSZ) electrolyte (which was previously verified against an analytical solution $^{4}$ ) has been incorporated into FLUENT as a user defined function (UDF). To validate the UDF, it is applied to a simple model (Figure 3-8) of a plane-parallel medium with temperatures fixed at the top and bottom. The results from the UDF are compared to results from the discrete ordinates (DO) method.

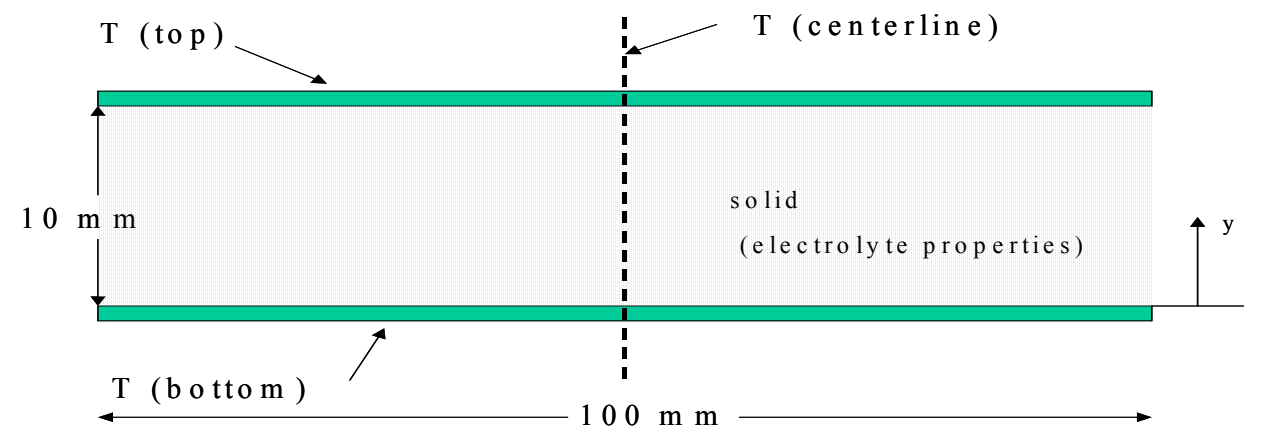

Figure 3-8: Model used to validate the 2-flux UDF in FLUENT.

First, the 2-flux UDF was compared to the DO method for various optical thicknesses in a case where radiative heat flux was dominant over conductive heat flux (by an order of magnitude). This was done to ensure that any problems with the code or formulation were not masked by other modes of heat transfer. A gray, non-scattering medium, with refractive index $\mathrm{n}=1.8$, between isothermal black plates was considered.

\footnotetext{
${ }^{4}$ Modest, M., Radiative Heat Transfer, $1{ }^{\text {st }}$ Edition, McGraw-Hill, 1993.
} 
Figure 3-9 (a-c) shows results for optical thicknesses $\left(\tau_{\mathrm{L}}\right)$ of $0.001,0.1$, and 1.0. The temperature of the upper and lower surfaces was $1200 \mathrm{~K}$ and $800 \mathrm{~K}$ respectively.

Next, the 2-flux UDF was compared to the DO method for the case of spectrallydependent extinction coefficient ( $\beta$ ). A 3-band model was implemented in the isotropically scattering medium (single scattering albedo $(\omega)=0.1$ ), bounded by diffuse, gray walls (emissivity $=0.9$ ). From experimental measurements of the extinction coefficient of the electrolyte material, the 3-band model used was:

$$
\beta=\left\{\begin{array}{cl}
160 \mathrm{~cm}^{-1} ; & 0.0<\lambda<3.5 \\
110 \mathrm{~cm}^{-1} ; & 3.5 \leq \lambda<5.0 \\
50 \mathrm{~cm}^{-1} ; & 5.0 \leq \lambda<\infty
\end{array}\right\}
$$

which, for electrolyte thickness of $30 \mu \mathrm{m}$, corresponds to $\tau_{\mathrm{L}}$ of $0.48,0.33$, and 0.15 . This model was solved, once again, for the case in which radiation was the dominant heat transfer mode (Figure 3-9d), showing excellent agreement between the DO method and the 2-flux UDF.

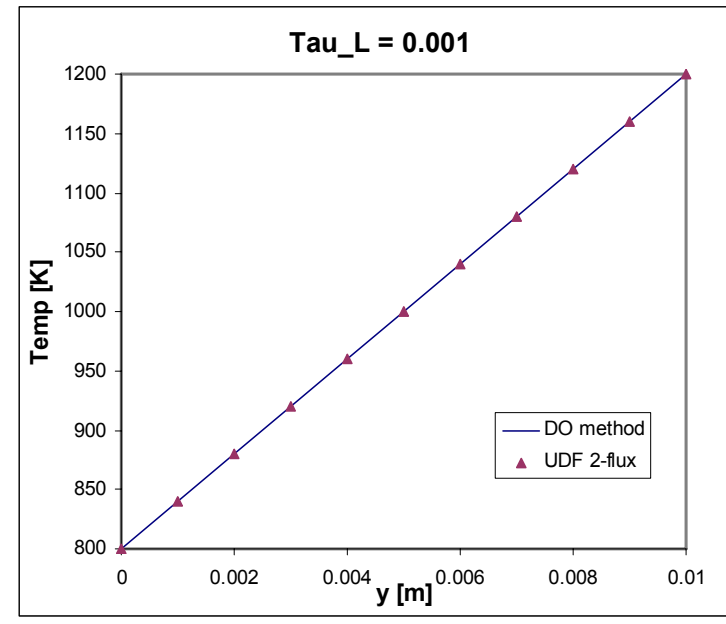

(a)

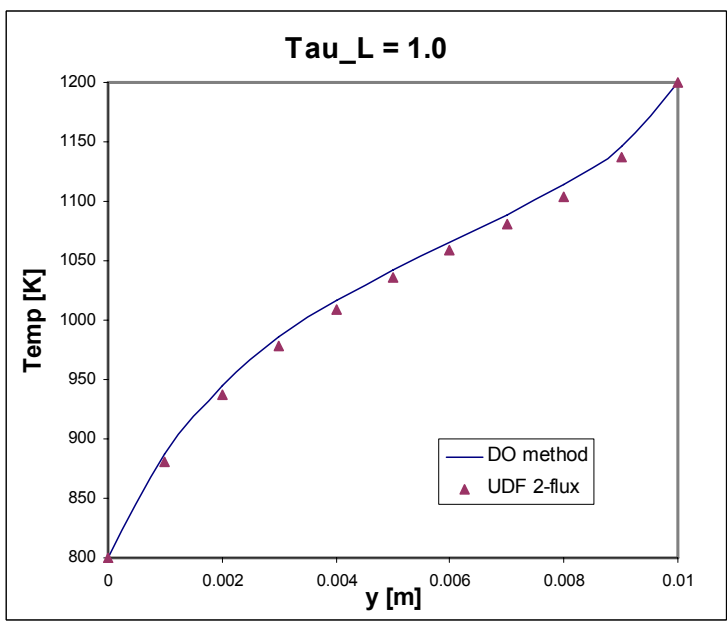

(c)

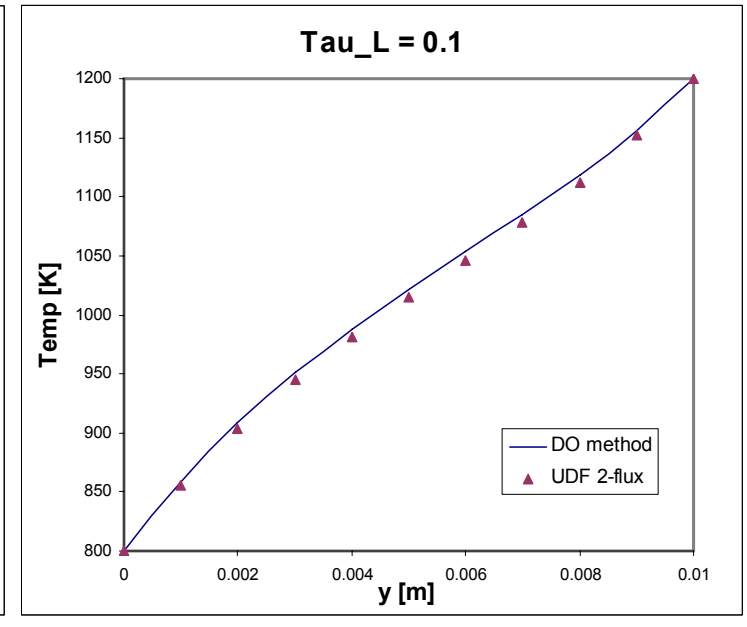

(b)

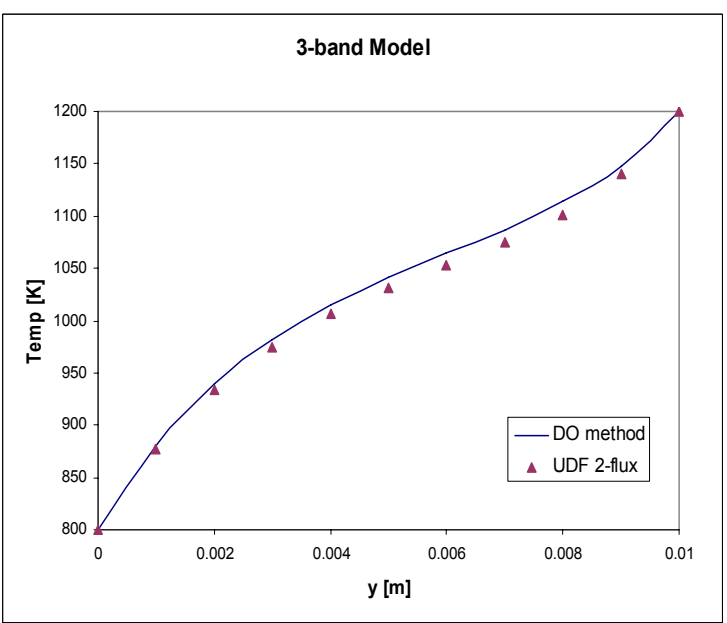

(d) 
Figure 3-9: (a-c) Comparison of temperature distributions in a plane-parallel, gray, non-scattering medium of various optical thickness $\left(\tau_{\mathrm{L}}\right)$ bounded by black, isothermal plates. (d) Results for 3-band model implementation of spectral-dependent extinction coefficient.

\subsubsection{Implementation of 2-flux method in SOFC model}

Implementation of the 2-flux UDF into the SOFC model requires adaptation of the code to the 3-D electrolyte "layer". Because the current formulation for calculating the heat flux on a single element within the layer depends on the temperature in surrounding cells, the entire 3-D temperature field must be retrieved from FLUENT and broken down into 1-D arrays. Then, once the divergence of the heat flux is calculated and added to the energy equation as a source term (using the DEFINE_SOURCE macro), the heat flux at the boundaries must be returned to FLUENT (using the DEFINE HEAT FLUX macro). This is required because FLUENT only calculates the diffusive heat flux across boundaries and interfaces when no radiation models are activated.

To verify that the 2-flux UDF is applicable to 3-D geometries and correctly defines the heat flux across interfaces, a simple model of three layers was solved (Figure 3-10). The upper surface of the top layer was maintained at $900 \mathrm{~K}$ and the lower surface of the bottom layer was maintained at $800 \mathrm{~K}$. All other external boundaries were adiabatic. The 2-flux UDF source term was added to the elements in the middle layer, and the radiative heat flux calculated by the UDF was imposed at the interfaces between layers. The top and bottom layers did not participate in radiation. The area weighted average heat flux from the top and bottom surface was compared to results from implementation of the DO method (Figure 3-11). For optical thickness of 0.1-1.0 (range of the YSZ electrolyte), the 2-flux UDF is reasonably accurate. The effect of mesh size in the y-direction (the only direction of radiative heat transfer, according to this formulation of the 2-flux approximation) is an area of concern. In the SOFC model, the electrolyte is very thin (approx. 15-30 $\mu \mathrm{m}$ ) and only 2 or 3 nodes in the y-direction are used. (The case from Figure 3-9 used 11 nodes and the case below used 6 nodes. The physical dimensions of elements are not as important to this analysis as optical thickness of each element). Although the UDF is sensitive to mesh size (due to numerical integration of emissive power over optical length), it is believed that the UDF will provide accurate solutions within the electrolyte layer because relatively low temperature gradients in the $y$ - direction are expected.

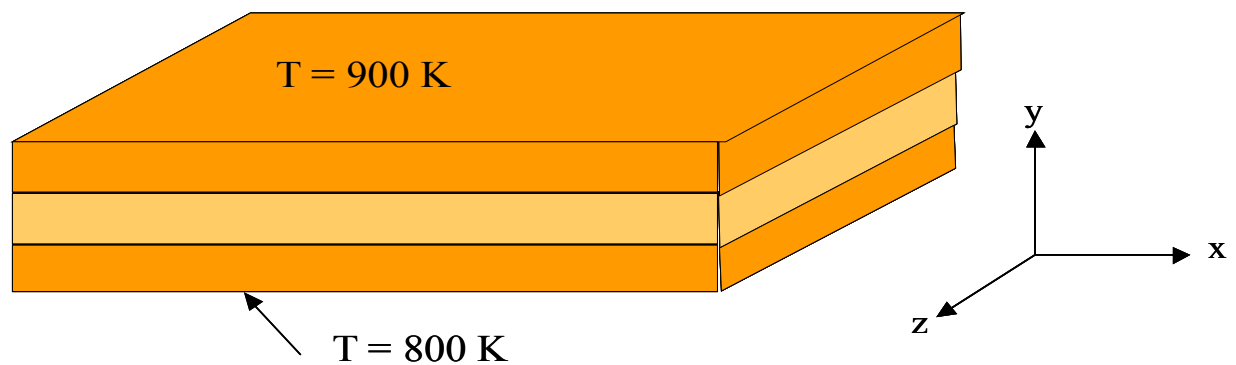

Figure 3-10: 3-D model used to validate 2-flux UDF. 


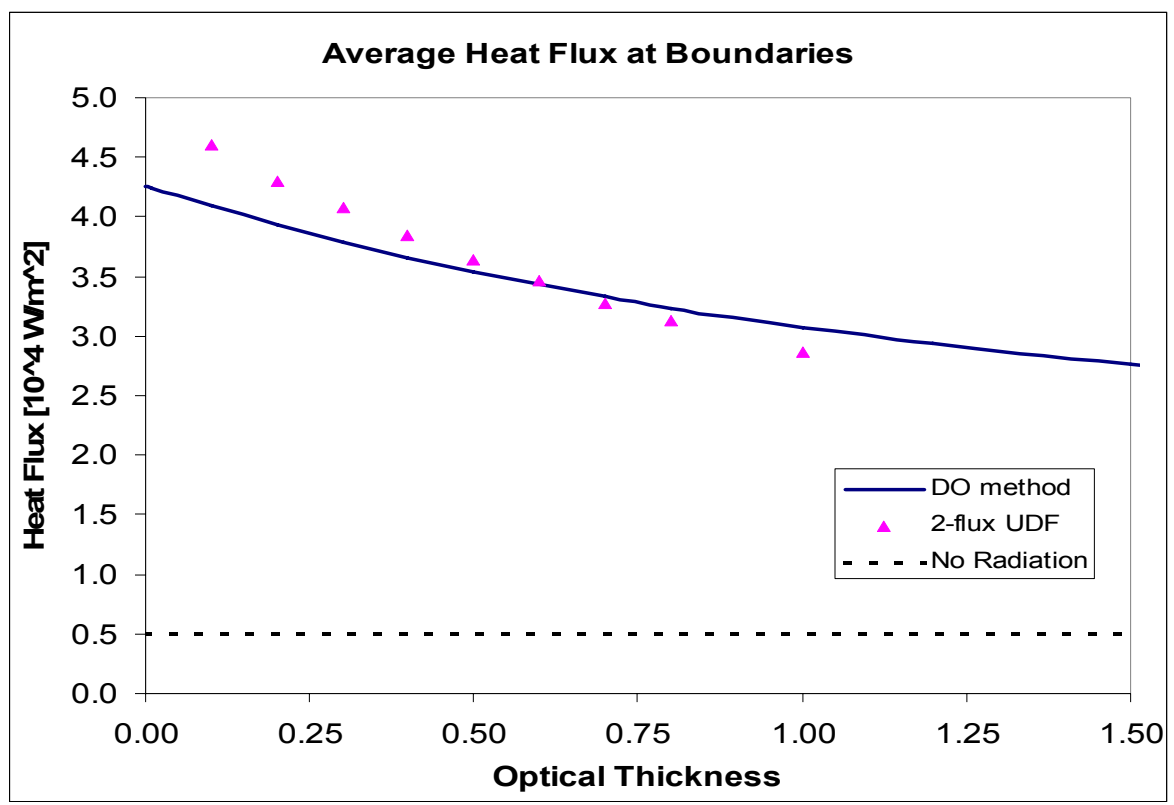

Figure 3-11: Comparison of total heat flux from top and bottom surfaces, as calculated by (1) activating DO model in FLUENT, and (2) addition of source term from 2-flux UDF. The dotted line is diffusive heat flux only (no radiation).

\subsubsection{Concluding Remarks}

The 2-flux UDF has been validated and implemented into the SOFC model. Results from this model will be obtained shortly, yielding a quantitative assessment of the effects of radiation on the SOFC model, and an overall comparison of the 2-flux method vs. DO method for this application.

\subsection{Constitutive Models for Applications of the Two-temperature Non-equilibrium Models}

Transient modeling of the SOFC unit cell is a prerequisite to mitigating thermomechanical failure caused by thermal gradients and cycling at start-up/shut-down. At start-up, it is desirable to heat the cell as quickly as possible under the constraint of some maximum allowable temperature gradient. Thermal modeling will focus on correlating the heating rate and the observed temperature gradients, and results will be presented in a manner that easily yields this information for the cell designed.

Three models of increasing complexity and accuracy will be developed. The first two models assume that the cell is thermally thin, that is, the cell materials have high thermal diffusivity so thermal field development in the solid intimately follows that in the heating air. The third model relaxes this assumption and gives consideration to the thermal resistance and latency (thermal energy storage) of the cell materials. Results from these models will be compared to the transient solution obtained by the fully 3-D transient Fluent model to identify the "best" model featuring the least degree of complexity and computational expense yet resulting in sufficient accuracy of simulation results. 


\subsubsection{Formulation of first-order transient heating model}

A first approach to analyzing transient heating of the SOFC unit cell is to assume the cell materials are thermally thin and the cell unit cell is adiabatic. The cell is initially at some temperature, $T_{\text {initial }}$, and is heated by hot air piped into the air channel at a constant velocity, $u$, and at some temperature, $T_{h o t}$, which is a function of time. The governing equation for this problem (derived in Appendix E) is expressed as,

$$
\frac{\delta T}{\delta t}+u \frac{\delta T}{\delta z}=0
$$

where, $u$ is the velocity of the air in the air channel and $z$ is the flow direction. The boundary and initial conditions are,

$$
\begin{aligned}
& T(z=0, t)=T_{\text {hot }}(t) \\
& T(z, t=0)=T_{\text {initial }}(z)
\end{aligned}
$$

The method of characteristics is employed to obtain the following analytical solution,

$$
T(z, t)=\left\{\begin{array}{lll}
T_{\text {initial }}(z-u t) & \text { for } & z>u t \\
\mathrm{~T}_{\text {hot }}\left(t-\frac{z}{u}\right) & \text { for } & z \leq u t
\end{array}\right.
$$

Thus the time-dependent temperature gradients in the z-direction of the cell can be found if the initial temperature and inlet temperature functions are known. It will be assumed that the initial temperature is constant along the cell. Thus, the temperature gradients will depend only on the variation of the inlet temperature as function of time.

\section{Linear Temperature Sweep}

Given a transient inlet (hot) air temperature variation of the form,

$$
T_{\text {hot }}=T_{o}+A t,
$$

where $A$ is a constant, a and $T_{o}$ is equal to $T_{\text {initial, }}$, the temperature from eqn (3.26) becomes,

$$
T(z, t)=\left\{\begin{array}{lll}
T_{\text {initial }} & \text { for } \quad z>u t ; & 0 \leq t<\frac{z}{u} \\
T_{\text {initial }}+A\left(t-\frac{z}{u}\right) & \text { for } \quad z \leq u t ; & 0 \leq t \leq t_{\max }
\end{array}\right.
$$


where, $t_{\max }$ is the total time required to reach the desired steady-state operating temperature:

$$
t_{\max }=\frac{T_{\text {steady }}-T_{\text {initial }}}{A}+\frac{L_{z}}{u} .
$$

Thus, the resulting temperature gradient is,

$$
\frac{\delta T}{\delta z}= \begin{cases}0 & \text { for } \quad z>u t \\ -A / u & \text { for } \quad z \leq u t\end{cases}
$$

at every time instant of the heating process. Spatially-temporal development of the temperature profile is shown schematically in Figure 3-12.

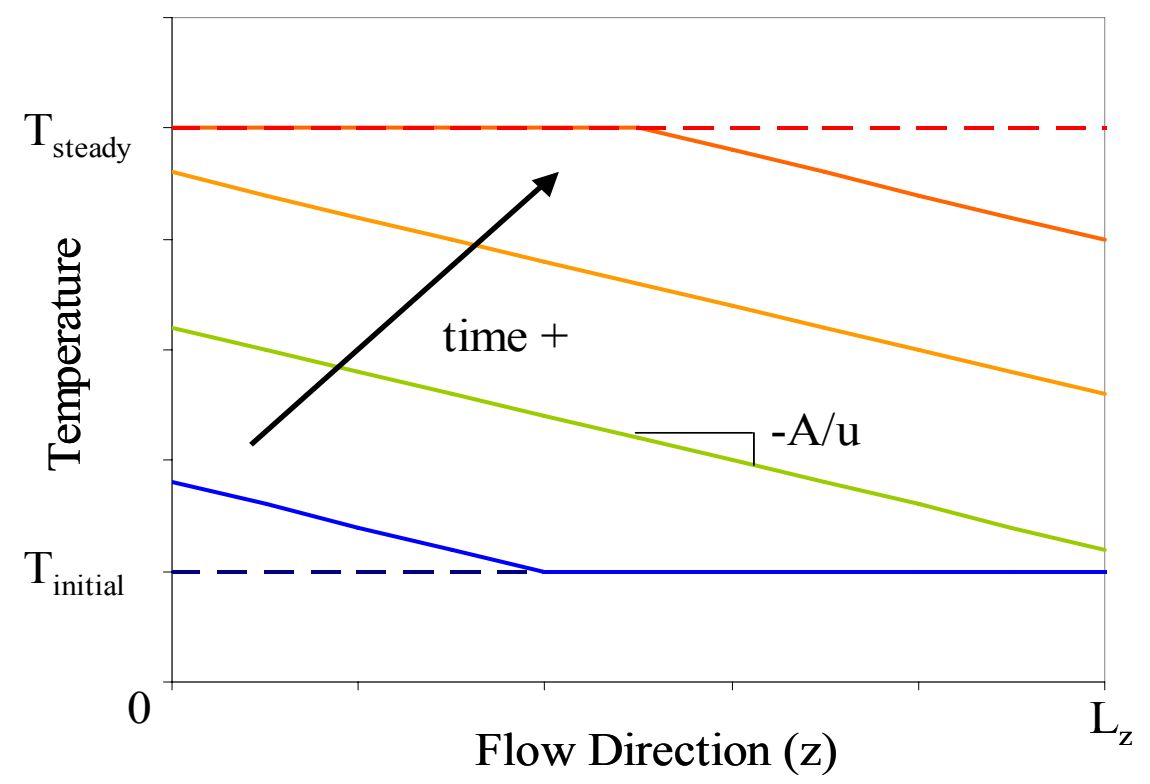

Figure 3-12: Development of the 1-D temperature profiles of the unit cell for a linear sweep inlet temperature boundary condition.

\section{Second-order Inlet Temperature}

Given temporal variation in an inlet hot air temperature of the form,

$$
T_{h o t}=T_{o}+B t^{2}
$$

the resulting temperature distribution is, 


$$
T(z, t)= \begin{cases}T_{\text {initial }} & \text { for } \quad z>u t ; \quad 0 \leq t<\frac{z}{u} \\ T_{\text {initial }}+B\left(t-\frac{z}{u}\right)^{2} & \text { for } \quad z \leq u t ; \quad 0 \leq t \leq t_{\max }\end{cases}
$$

with heating time to reach the steady-state,

$$
t_{\max }=\sqrt{\frac{T_{\text {steady }}-T_{\text {initial }}}{B}}+\frac{L_{z}}{u}
$$

The resulting temperature gradient is time-dependent and given by

$$
\frac{\delta T}{\delta z}=-\frac{2 B}{u}\left(t-\frac{z}{u}\right) \quad \text { for } \quad z \leq u t
$$

Upon inspection of Eq. (3.34), the magnitude of the temperature gradient increases monotonically with time. The shape of the temperature profile is shown in Figure 3-13.

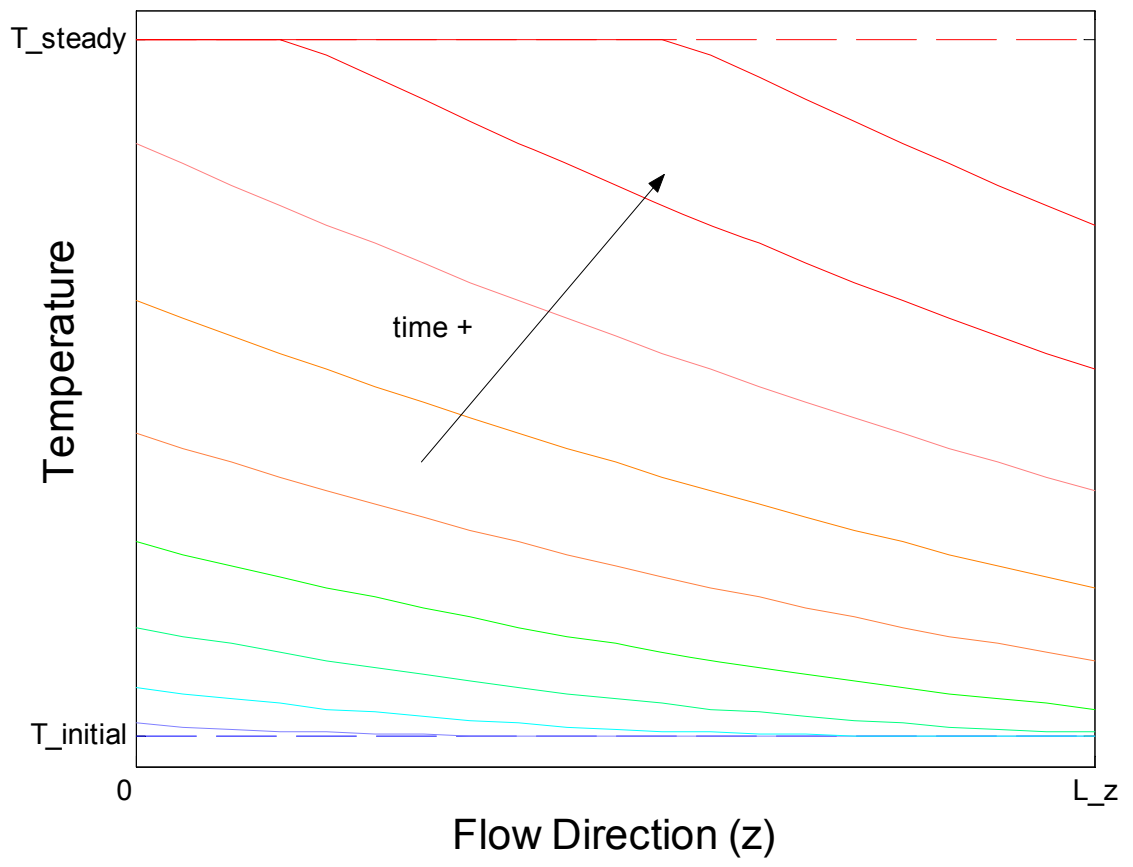

Figure 3-13: Development of the temperature profiles within the unit cell for a second order inlet temperature rise boundary condition.

Previously, the first order, purely convective transient heating model was formulated for linear sweep inlet temperature and second order inlet temperature functions. Here, results from these two cases are presented in terms of the key design 
criteria: time required for heating, and maximum temperature gradient developed in the cell.

\subsubsection{Further Development Formulation of first-order transient heating model}

The variables of interest in transient heating of the cell are the total time required for heating, and the maximum temperature gradient developed in the cell. Figure 3-14 is a map showing the temperature gradient versus required heating time for several inlet velocities and rates of temperature rise. For this particular case (linear sweep inlet temperature function), the initial temperature was taken to be $25^{\circ} \mathrm{C}$ and the final temperature $600^{\circ} \mathrm{C}$, with a channel length of $0.1 \mathrm{~m}$. The physical implication of is that to achieve lower heating times, with lower temperature gradients, the air stream must have an increasingly higher velocity and rate of temperature rise. In reality, practical and physical limits to this trend must exist, but the current model provides little or no insight into where those limits might be. However, since the model assumes infinitely quick response of the cell, it provides a most optimistic estimate of the heating time that will be required under the constraint of a maximum allowable temperature gradient.

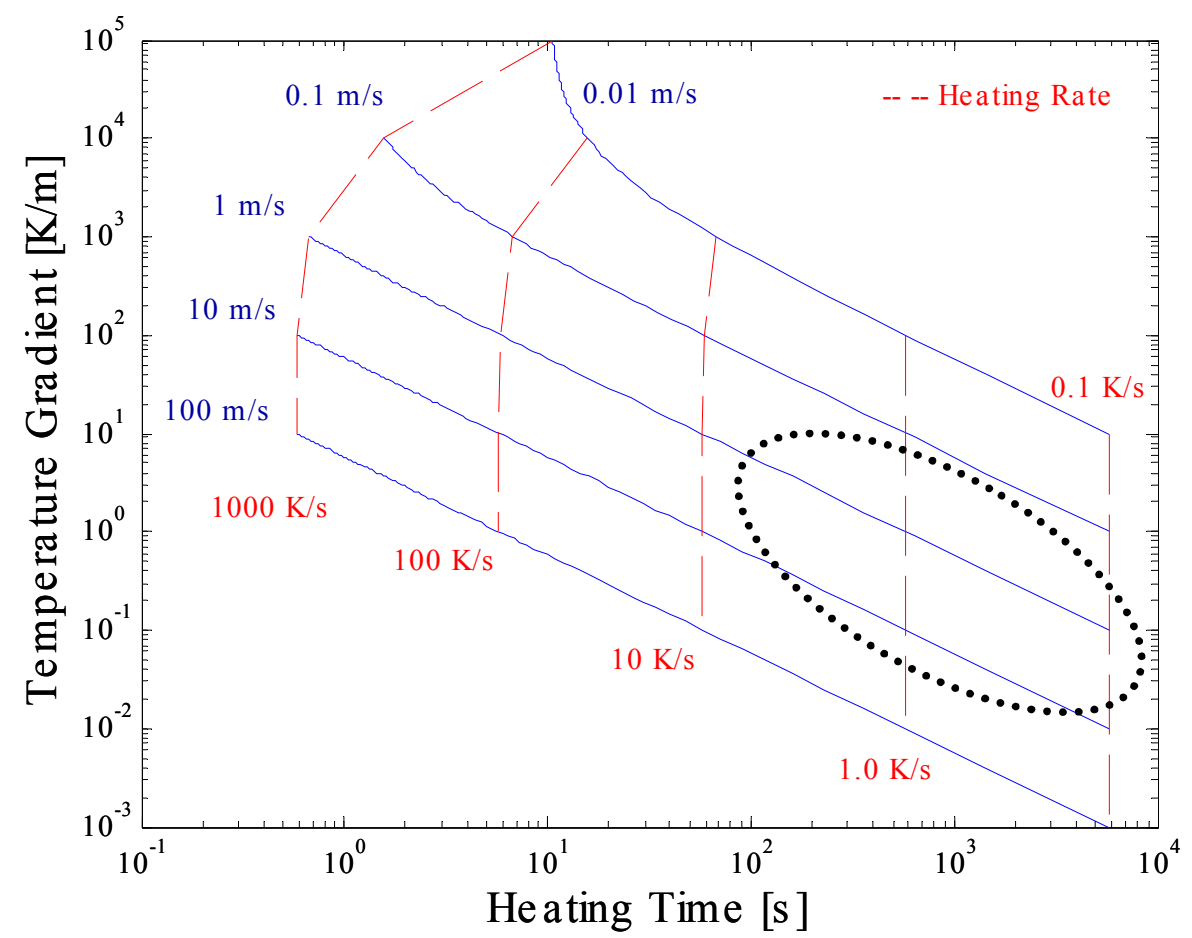

Figure 3-14: Maximum temperature gradient as a function of heating time for various air stream velocities.

Also, shown are various rates of temperature rise of the inlet air stream. The circled area represents physically realistic parameters that a system would be expected to operate under.

In a similar manner to the case above, the total required heating time is compared to the maximum temperature gradients for the case of a second order inlet temperature function. In Figure 3-15 below, results from this second case are superimposed over the map from Figure 3-14. In general, the curves are shifted in an undesirable direction from the case above, resulting in longer heating times and higher temperature gradients for 
similar sets of operating parameters. The reason for this is that the second order function has a lower rate of temperature rise at small times (i.e. wasting time), and then induces excessive temperature gradients as time increases.

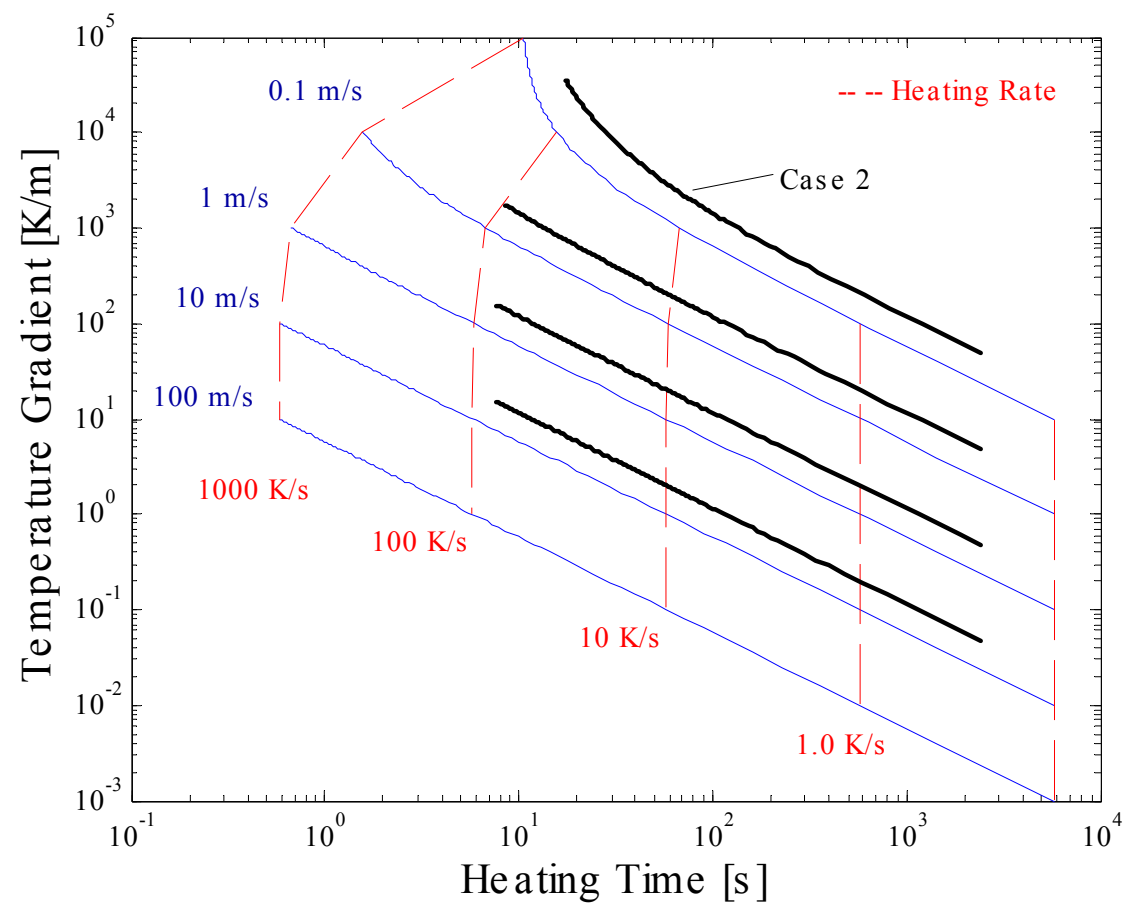

Figure 3-15: A comparison of temperature gradient versus heating time for case 1 and 2. The initial and final temperatures were $25^{\circ}$ and $600^{\circ} \mathrm{C}$.

Because the linear temperature sweep case results in lower heating times and temperature gradients, it will be discussed in more detail. In general, the following scaling relationship exists between the SOFC operating parameters:

$$
\frac{A t_{\max }}{\Delta T}=1+\frac{L_{z} A}{\Delta T u}
$$

In designing a transient heating scheme, it will be assumed that the air/fuel channel length, $L_{z}$, and required temperature rise, $\Delta T$, are given. The air stream velocity, $u$, and rate of temperature rise of the inlet air stream, $A$, are taken as the variables that can be controlled by the system operator. The variables that the designer will optimize are the total time to steady state, $t_{\max }$, (must be minimized for rapid start-up) and maximum temperature gradient, $\frac{\delta T}{\delta z}$ (must be below the threshold value imposed by thermomechanical failure considerations).

A special case can be derived by investigating the various time scales involved in transient heating under typical SOFC operating condtions. In particular, the time scale for air to travel from the inlet to exit of the channel is $L_{z} / u$, which is of the order $<1 \mathrm{~s}$ for cell length of $0.1 \mathrm{~m}$ and velocity greater than $0.1 \mathrm{~m} / \mathrm{s}$. On the other hand, the 
characteristic time for heating the cell, given by $\Delta T / A$, is likely to be of the order $>100$ $\mathrm{s}$, for a $1 \mathrm{~K} / \mathrm{s}$ rate of temperature rise and a total temperature rise of $500 \mathrm{~K}$. Thus, effects related to the length of the channel can be neglected if the following criteria is satisfied:

$$
\frac{L_{z} A}{\Delta T u}<<1
$$

which, in turn, reduces to,

$$
\frac{A t_{\max }}{\Delta T} \simeq 1
$$

Equation (3.37) constitutes a simple design rule, which is expected to apply to the vast majority of cases that would be of interest to SOFC system designers. The percent error introduced by this approximation is simply, $\frac{L_{z} A}{\Delta T u} x 100 \%$. Further, the relationship between temperature gradient, rate of temperature rise, and velocity,

$$
\frac{\delta T}{\delta z}=\frac{A}{u}
$$

showing that increasing velocity of the air stream is generally favorable. It is up to the designer to use eqns. (3.37) and (3.38) to minimize heating time while maintaining the temperature gradient below an allowed failure limit. In general, the following design rules apply: higher air velocities tend to decrease temperature gradients, and higher heating rates tend to decrease the total time requirements for startup. The magnitude of these two parameters will likely be limited by physical capabilities of the plant, long before temperature gradients become a thermo-mechanical failure issue. It should be noted that although the discussion thus far has focused on startup, this model also applies to shutdown, which mirrors the results of startup. Also, it should be emphasized that all the results are valid only in the limit of thermally thin cell stack analysis.

In view of the approximate nature of the above analysis, it is reasonable to suggest that this simplistic model might provide an envelope for solutions of future models. Figure 3-16 shows what this envelope looks like, plotting the results against temperature gradient normalized by the required temperature rise. The limiting behavior described is clearly seen in this plot as a straight vertical line, and fortunately this behavior represents the region of interest for transient startup/shutdown. The validity and usefulness of the current model, will be addressed after the next generation of improved models will have been formulated and implemented. 


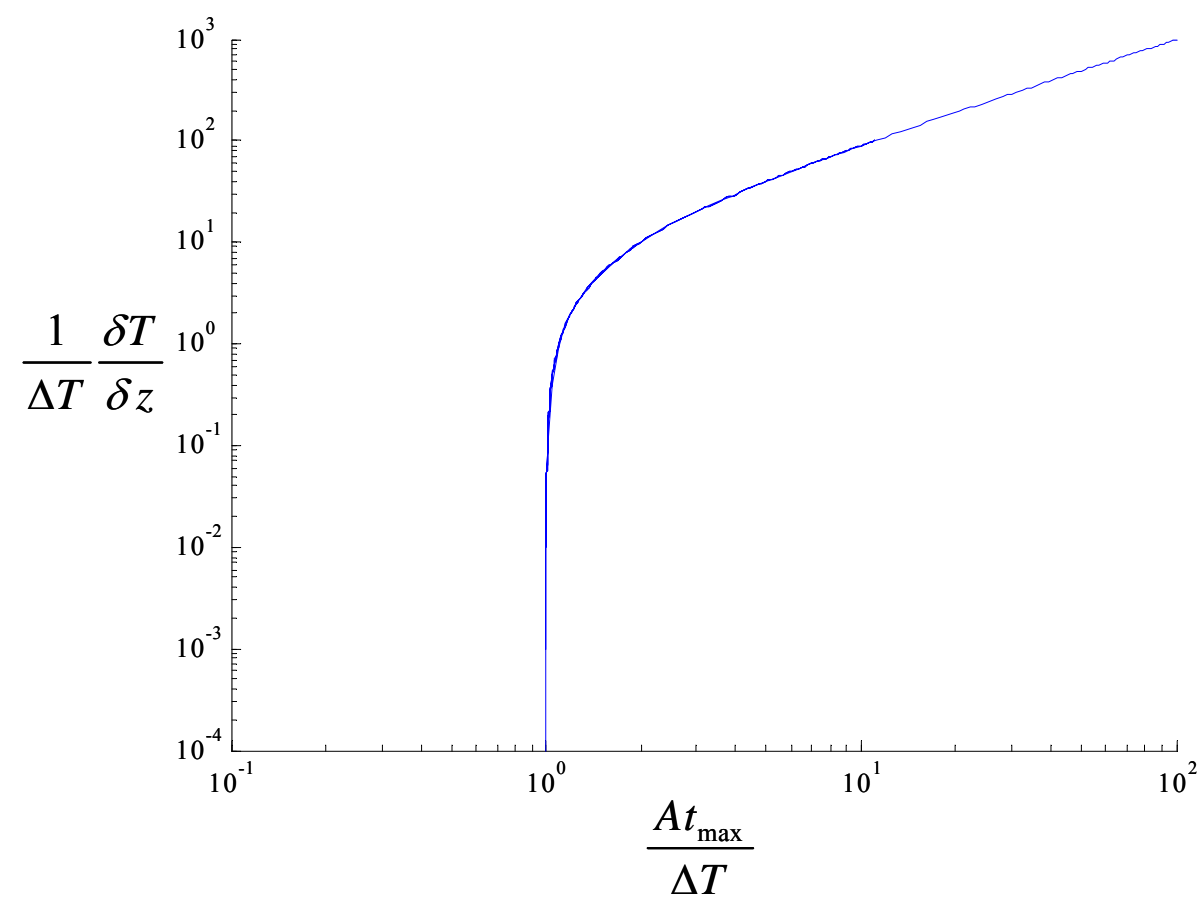

Figure 3-16: Solutions of the first order purely convective heating model for a wide range of operating conditions and parameters relevant to SOFC startup scenarios.

\subsubsection{Concluding Remarks}

The first and simplest 1-D transient heating model has been formulated and implemented, and results have been obtained and discussed for two inlet temperature functions. A framework for presenting transient analysis results in a clear and concise manner has been developed, emphasizing the variables of interest from a design/optimization perspective and presenting the results of this first model. Before judgments are made concerning the validity of the model, more detailed and accurate models will be developed and implemented for comparison.

\subsection{Second-order transient heating model}

\subsubsection{Formulation and solution of second-order transient heating model}

In the second-order model currently under development, this assumption is relaxed; however, the assumption of local thermal equilibrium between the hot air flowing in the air channel and the cell materials is applied to simplify the analysis. This allows the governing equations to be solved analytically, while providing more physically realistic results, as compared to the simplest first-order model, by accounting for thermal energy storage of the cell materials.

The formulation begins by writing the energy conservation equation for each layer and component of the cell. The governing energy conservation equations for the most general, non-equilibrium model are a set of PDE's, coupled by source terms in the 
right hand side that depend on the local temperature difference between adjacent (thermally interacting) layers of the cell unit cell. These are:

InterConnect ${ }_{1}$ :

$$
\left(\rho C_{p} A\right)_{I C_{1}} \frac{\partial T_{I C_{1}}}{\partial t}=(k A)_{I C_{1}} \frac{\partial^{2} T_{I C_{1}}}{\partial z^{2}}+h P_{g-I C_{1}}\left(T_{g}-T_{I C_{1}}\right)-\frac{P_{I C_{1}-C}}{R_{I C_{1}-C}}\left(T_{I C_{1}}-T_{C}\right)
$$

Air Channel (gas):

$$
\left(\rho c_{p} A\right)_{g}\left[\frac{\partial T_{g}}{\partial t}+u \frac{\partial T_{g}}{\partial z}\right]=(k A)_{g} \frac{\partial^{2} T_{g}}{\partial z^{2}}-h P_{g-C}\left(T_{g}-T_{C}\right)-h P_{g-I C_{1}}\left(T_{g}-T_{I C_{1}}\right)
$$

Cathode:

$$
\left(\rho c_{p} A\right)_{C} \frac{\partial T_{C}}{\partial t}=(k A)_{C} \frac{\partial^{2} T_{C}}{\partial z^{2}}+h P_{g-C}\left(T_{g}-T_{C}\right)+\frac{P_{I C_{1}-C}}{R_{I C_{1}-C}}\left(T_{I C_{1}}-T_{C}\right)-\frac{P_{C-E}}{R_{C-E}}\left(T_{C}-T_{E}\right)
$$

Electrolyte:

$$
\left(\rho c_{p} A\right)_{E} \frac{\partial T_{E}}{\partial t}=(k A)_{E} \frac{\partial^{2} T_{E}}{\partial z^{2}}+\frac{P_{C-E}}{R_{C-E}}\left(T_{C}-T_{E}\right)-\frac{P_{E-A}}{R_{E-A}}\left(T_{E}-T_{A}\right)
$$

Anode:

$$
\left(\rho c_{p} A\right)_{A} \frac{\partial T_{A}}{\partial t}=(k A)_{A} \frac{\partial^{2} T_{A}}{\partial z^{2}}+\frac{P_{E-A}}{R_{E-A}}\left(T_{E}-T_{A}\right)-h P_{f-A}\left(T_{f}-T_{A}\right)-\frac{P_{A-I C_{2}}}{R_{A-I C_{2}}}\left(T_{A}-T_{I C_{2}}\right)
$$

FuelChannel:

$$
\left(\rho c_{p} A\right)_{\text {fuel }} \frac{\partial T_{\text {fuel }}}{\partial t}=(k A)_{\text {fuel }} \frac{\partial^{2} T_{\text {fuel }}}{\partial \mathbf{z}^{2}}+h P_{f-A}\left(T_{f}-T_{A}\right)+h P_{f-I C_{2}}\left(T_{f}-T_{I C_{2}}\right)
$$

InterConnect ${ }_{2}$ :

$$
\left(\rho c_{p} A\right)_{I C_{2}} \frac{\partial T_{I C_{2}}}{\partial t}=(k A)_{I C_{2}} \frac{\partial^{2} T_{I C_{2}}}{\partial z^{2}}+\frac{P_{A-I C_{2}}}{R_{A-I C_{2}}}\left(T_{A}-T_{I C_{2}}\right)-h P_{f-I C_{2}}\left(T_{f}-T_{I C_{2}}\right)
$$

It is assumed that each layer is at a uniform (average) temperature across the thickness of the layer as well as the thermophysical properties are constant for each layer of the modeled SOFC unit cell. The boundary and initial conditions are, respectively,

$\underline{\text { B.C.'s }}$

$$
\begin{aligned}
& T_{g}(0, t)=f(t) ; \quad \frac{\partial T_{g}}{\partial z}(L, t)=\frac{\partial T_{I C}}{\partial z}(0, t)=\frac{\partial T_{I C}}{\partial z}(L, t)=\frac{\partial T_{C}}{\partial z}(0, t)=\frac{\partial T_{C}}{\partial z}(L, t)=0 \\
& \frac{\partial T_{E}}{\partial z}(0, t)=\frac{\partial T_{E}}{\partial z}(L, t)=\frac{\partial T_{A}}{\partial z}(0, t)=\frac{\partial T_{A}}{\partial z}(L, t)=\frac{\partial T_{f}}{\partial z}(0, t)=\frac{\partial T_{f}}{\partial z}(L, t)=0
\end{aligned}
$$

\section{I.C.'s}

$$
T_{g}(z, 0)=T_{I C}(z, 0)=T_{C}(z, 0)=T_{E}(z, 0)=T_{A}(z, 0)=T_{f}(z, 0)=T_{o}
$$

This model is the third-order model, as was described in the proposal, which can be only numerically solved, as it is going to be done in the future. For now, however, the 
assumption of the local thermal equilibrium between layers is employed in order to simplify the governing equations. To accomplish this task correctly (i.e., without violating energy conservation even locally), the following procedure is employed: First, all equations are added together, which cancels the coupling terms. Also, because of local thermal equilibrium assumptions, the temperatures of different layers are the same (by definition of the local thermal equilibrium), leading to the following governing equation describing transient heating dynamics of the cell unit cell:

$$
\frac{\partial T}{\partial t}+u_{e f f} \frac{\partial T}{\partial z}=\alpha_{e f f} \frac{\partial^{2} T}{\partial z^{2}}
$$

subject to the boundary and initial conditions,

$$
\begin{aligned}
& T(0, t)-\sigma_{\text {eff }} \frac{\partial T}{\partial z}(0, t)=f(t) ; \quad \frac{\partial T}{\partial z}(L, t)=0 \\
& T(z, 0)=T_{o}
\end{aligned}
$$

where the effective velocity and thermal diffusivity are defined as,

$$
\begin{aligned}
& u_{\text {eff }}=\frac{\left(\rho c_{p} A\right)_{g}}{\left(\rho c_{p} A\right)_{I C_{1}}+\left(\rho c_{p} A\right)_{g}+\left(\rho c_{p} A\right)_{C}+\left(\rho c_{p} A\right)_{E}+\left(\rho c_{p} A\right)_{A}+\left(\rho c_{p} A\right)_{f}+\left(\rho c_{p} A\right)_{I C_{2}}} \cdot u \\
& \alpha_{e f f}=\frac{(k A)_{I C_{1}}+(k A)_{g}+(k A)_{C}+(k A)_{E}+(k A)_{A}+(k A)_{f}+(k A)_{I C_{2}}}{\left(\rho c_{p} A\right)_{I C_{1}}+\left(\rho c_{p} A\right)_{g}+\left(\rho c_{p} A\right)_{C}+\left(\rho c_{p} A\right)_{E}+\left(\rho c_{p} A\right)_{A}+\left(\rho c_{p} A\right)_{f}+\left(\rho c_{p} A\right)_{I C_{2}}}
\end{aligned}
$$

and,

$$
\sigma_{\text {eff }}=\frac{(k A)_{I C_{1}}+(k A)_{C}+(k A)_{E}+(k A)_{A}+(k A)_{f}+(k A)_{I C_{2}}}{\left(\rho C_{p} A\right)_{g} u}
$$

The non-dimensional form of eqn. (3.41) is,

$$
\frac{\delta T}{\delta t}+\frac{\delta T}{\delta z}=\frac{1}{P e} \frac{\delta^{2} T}{\delta z^{2}}
$$

with the Peclet number $(\mathrm{Pe})$ defined in terms of the effective air velocity and thermal diffusivity of the cell,

$$
\frac{1}{P e}=\frac{\alpha_{e f f}}{u_{e f f} L}=\frac{1}{\operatorname{RePr}}
$$


The Peclet number represents the ratio of advection to conduction of thermal energy in the stack. It is the non-dimensional group expressed in terms of the effective transport (air velocity) and thermophysical properties (thermal diffusivity), which govern the transient dynamics of the cell stack. In non-dimensionalizing the governing equation, the following scales have been used for temperature, length and time, respectively,

$$
T=\frac{T^{*}}{T_{o}} ; \quad z=\frac{z^{*}}{L} ; \quad t=\frac{t^{*}}{(L / u)}
$$

The analytical solution to eqn. (3.45) is a series solution, which is derived in some detail in Appendix F.

\subsubsection{Results of second-order transient heating model}

In the transient analysis of the unit cell, the three quantities of interest are temperature gradients within the PEN materials, time rate of change of temperature within these materials, and total time required to reach the desired operating temperature. At first, we look at the case when the cell, initially at some constant temperature, is heated by flowing hot air into the air channel at a constant velocity, while the temperature of the air stream is increased linearly by some rate, $K^{*}$. The second-order model, that simulates this process, has been solved in non-dimensional form in terms of the Peclet number, $P e$, final temperature, $T_{f}$, and rate of inlet temperature rise.

Figure 3-17 is a dimensionless plot of time-varying temperature profiles within the cell, from a cold start-up to a final operating temperature. Temperature, length, and time are scaled by initial temperature, length of the channel, and residence time of the air in the channel (given by length divided by effective velocity), respectively,

$$
T=\frac{T^{*}}{T_{o}} ; \quad z=\frac{z^{*}}{L} ; \quad t=\frac{t^{*}}{\left(L / u_{e f f}\right)}
$$

where the superscript $*$ indicates a dimensional variable. As expected, the maximum temperature gradients occur near the inlet and decrease along the flow direction.

Because the maximum temperature gradient plays a role in the development of thermal stresses, it is calculated and shown in Figure 3-18. The Peclet number, Pe, is a non-dimensional group that gives the ratio of advection of thermal energy by flow in the air channel, to diffusion of thermal energy in the cell materials. For realistic cell operating conditions, we estimated that $P e$ varies between 0.1 and 10. This is a transition region between diffusion dominated heat transfer at small $P e$ and advection-dominated regimes at high $\mathrm{Pe}$. (The latter has already been considered, to some extent, in the firstorder, thermally thin model.)

An interesting feature of the profiles in Figure 3-18 is that the limiting value of temperature gradient for a given rate of inlet temperature rise, $K$, is approached at high $P e$. We can say in general that for any Pe number case, 


$$
\begin{aligned}
& \frac{\partial T}{\partial z} \leq K \quad(\text { nondimensional }) \\
& o r \\
& \frac{\partial T^{*}}{\partial z^{*}} \leq \frac{K^{*}}{u_{e f f}} \quad(\text { dimensional })
\end{aligned}
$$

This looks very familiar, and in fact, compares favorably to the result from the first-order model at high Pe,

$$
\frac{\partial T^{*}}{\partial z^{*}}=\frac{K^{*}}{u}
$$

Notice, however, that the velocity has been replaced with the effective velocity, which is several orders of magnitude lower than the real velocity, and thus gives a temperature gradient several orders higher. As expected, the temperature gradient becomes lower with decreasing Pe. As the velocity decreases, the dynamics of the heating process is dominated by thermal diffusion in the cell materials which tends to smear the sharp temperature gradients. There is caveat to this conclusion - although the thermal-diffusion limited operation with smaller flow velocity (Peclet number) is favorable from the thermal gradient minimization prospective, the total time required to reach the steadystate operating temperature is also increasing with a decrease in the heating air velocity (Peclet number). Thus, there must exist an optimum heating regime that results in the fastest possible heating of the stack, yet does not produce unacceptable temperature gradients leading to thermomechanical cell failure.

The next quantity of interest that arises in transient operation of a cell is the time rate of change of temperature (temperature derivative in respect to time) in cell materials. This is important because the cell is composed of layers of different material with different thermal expansion coefficients and characteristic time scales for creep. If the temperature derivative exceeds some value, interfacial stress due to thermal expansion mismatch can cause delamination. The temperature derivative has negligible dependence on position in the z-direction. Figure 3-19 shows the maximum time-derivative of the temperature in the cell for various rates of inlet temperature rise. Notice the lack of dependence on $\mathrm{Pe}$ and the one-to-one relationship between temperature derivative and rate of inlet temperature rise. This yields the simple relationship,

$$
\begin{aligned}
& \frac{\partial T}{\partial t} \sim K \quad \text { (dimensionless) } \\
& \text { or, } \\
& \frac{\partial T^{*}}{\partial t^{*}} \sim K^{*} \quad \text { (dimensional) }
\end{aligned}
$$

which is particularly useful because of its simplicity. This result is a direct outcome of the local thermal equilibrium assumption that we used in deriving the second-order model. The slight deviation from the dynamics occurs only at higher values of the K-parameter, when Pe-dependance becomes noticeable. 
The results for total time required to achieve operating temperature are shown in Figure 3-20. on a linear scale vs. rate of inlet temperature rise for various final temperatures. The final temperature is scaled by the initial temperature, and time is scaled as given by eqn. (3.48). On this plot it is difficult to see the $P e$ dependence of the total time requirement. Figure 3-21 is the same plot on a log-log scale, which shows this more clearly. It is expected that the total time should depend on the final temperature and the rate of temperature rise in the following fashion,

$$
\begin{aligned}
& t_{\max } \sim \frac{T_{f}-1}{K} \quad \text { (dimensionless) } \\
& \text { or, } \\
& t_{\max }^{*} \sim \frac{T_{f}^{*}-T_{o}^{*}}{K^{*}} \quad \text { (dimensional) }
\end{aligned}
$$

which assumes the residence time scale of flow passing through the channel is negligible compared to the overall time scale to reach operating temperature. This would occur when either the flow velocity is very high or thermal diffusivity of the stack materials is very low, leading to the high Peclet number limit. However, this is not the case if effective velocity (Peclet number) is moderate, which yields a characteristic time for flow on the order of $10^{3}$ seconds does not appropriately describe the maximum time behavior. Clearly, there is a deviation in curves for different Peclet number cases at higher heating rates (K-values), with higher Peclet numbers leading to shorter start-up time (lower $\mathrm{t}_{\mathrm{max}}$ ). Further analysis of this dynamics is required to fully understand and predict the time requirements for startup and shutdown of the SOFC, and it will be reported in the followup reports.

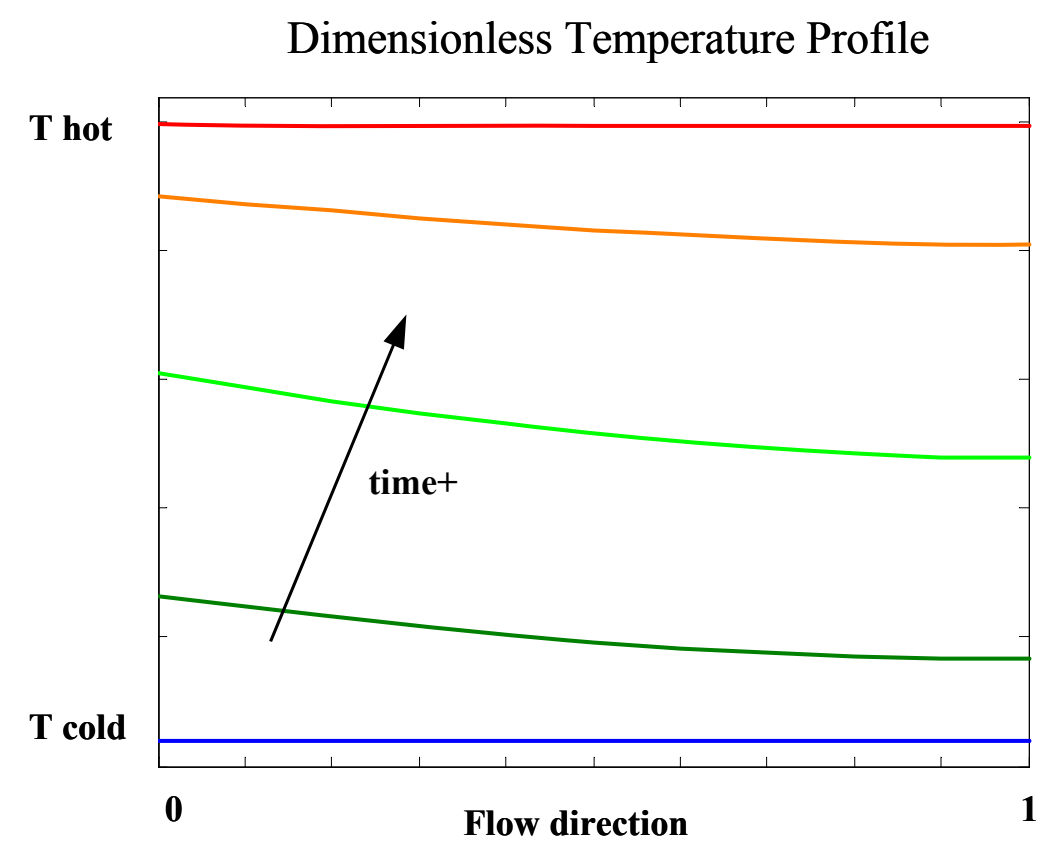


Figure 3-17: Time-varying dimensionless temperature profiles from an initial temperature, $T$ cold, to a final temperature, $T$ hot. The arrow shows the direction of increasing time.

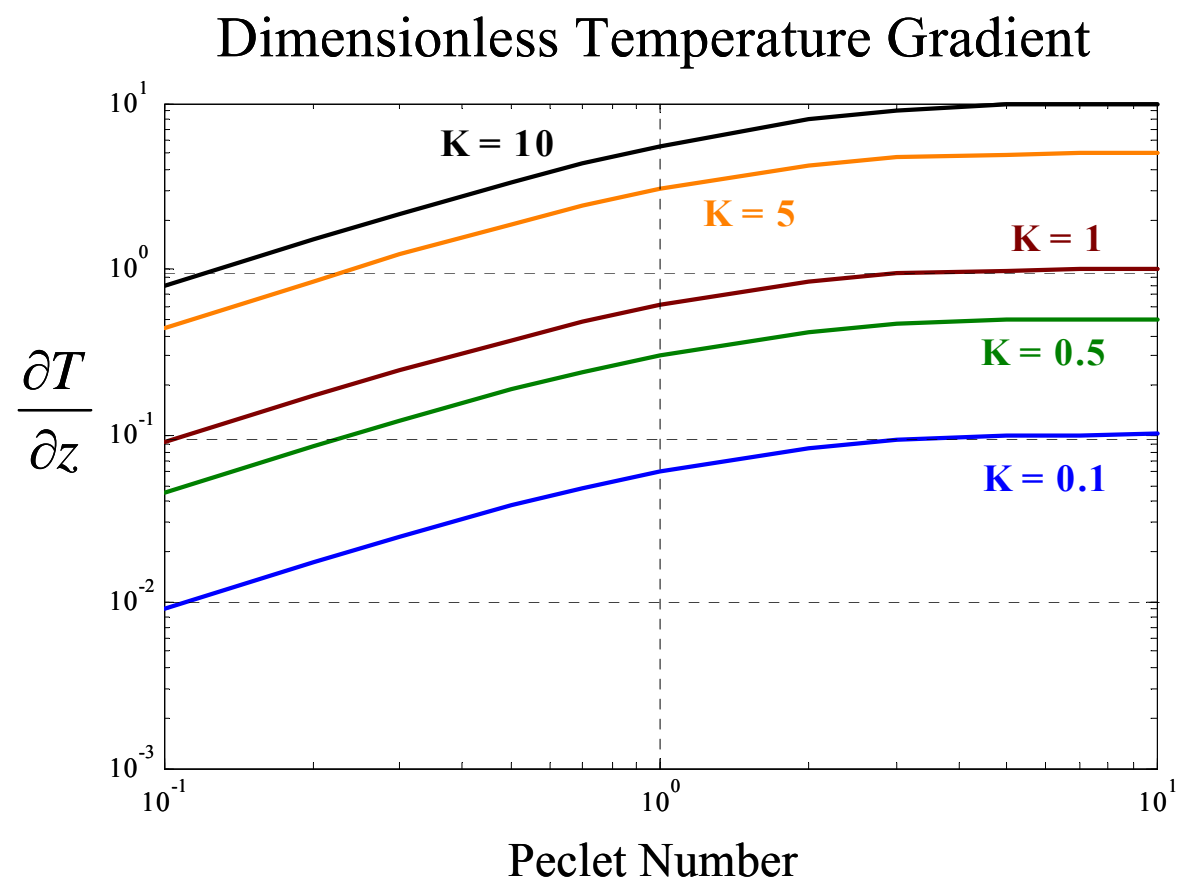

Figure 3-18: Temperature gradient vs. Peclet number for various values of rate of inlet temperature rise. 


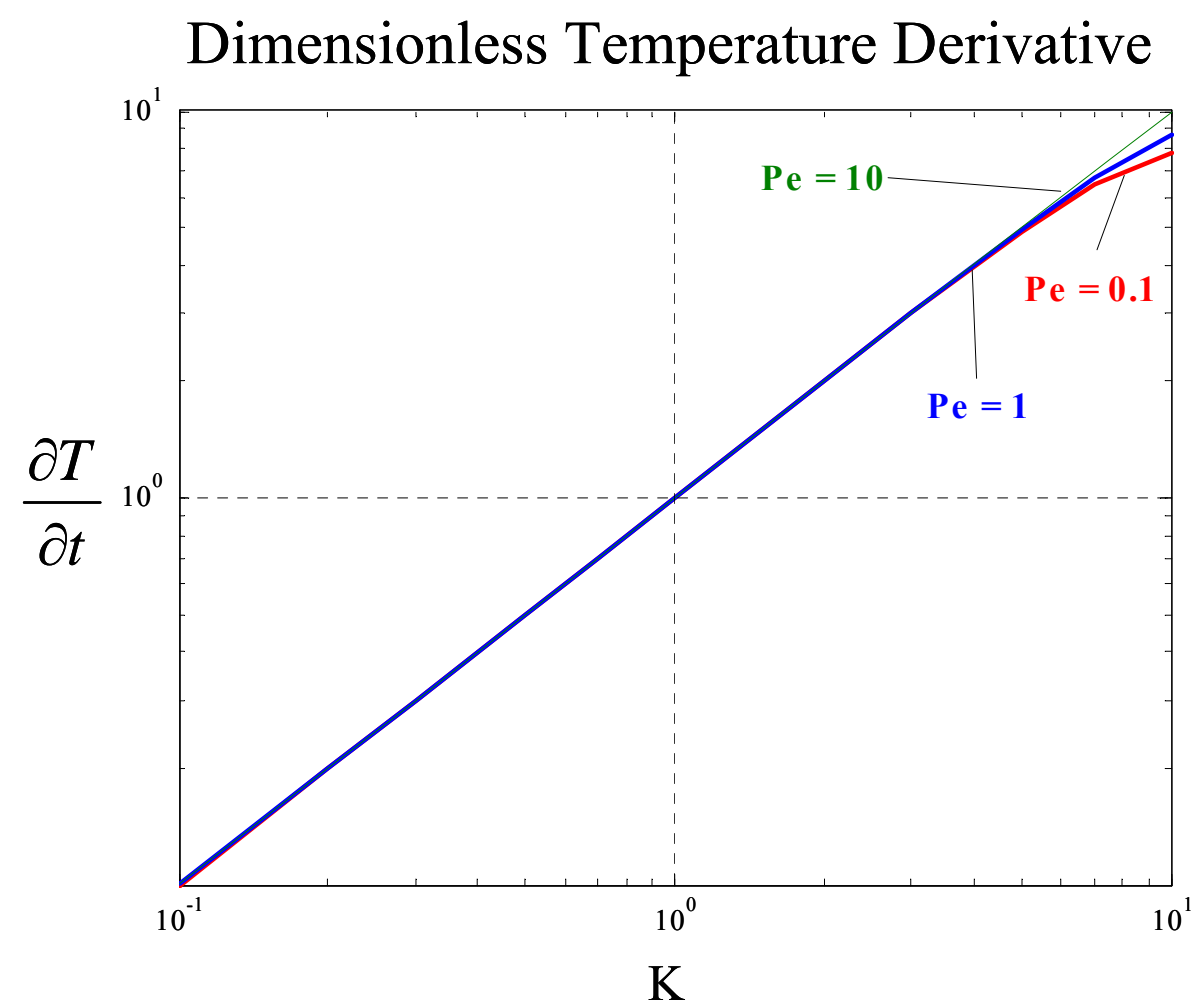

Figure 3-19: Temperature derivative vs. rate of inlet temperature rise for various values of Peclet number.

\section{Total Time for Startup/Shutdown}

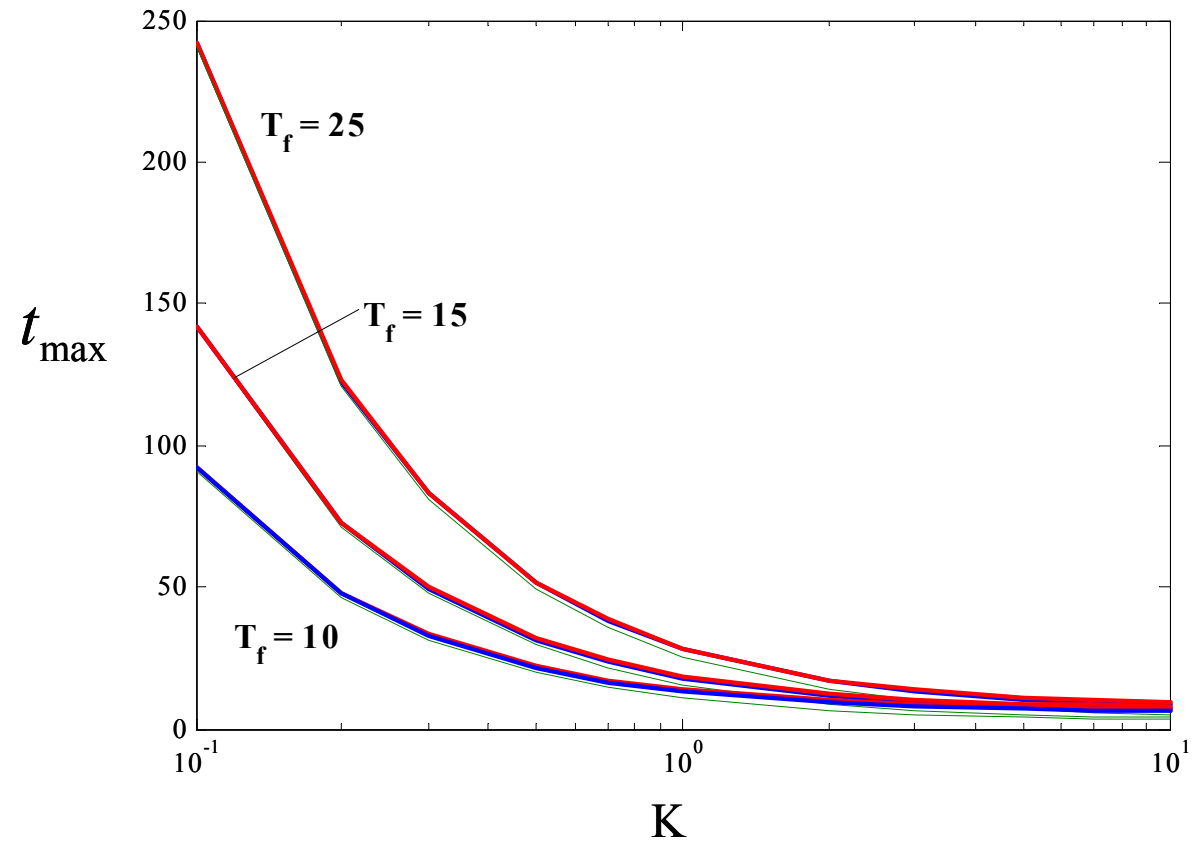


Figure 3-20: Time required to reach operating temperature vs. rate of inlet temperature rise for various final temperatures (dimensionless).

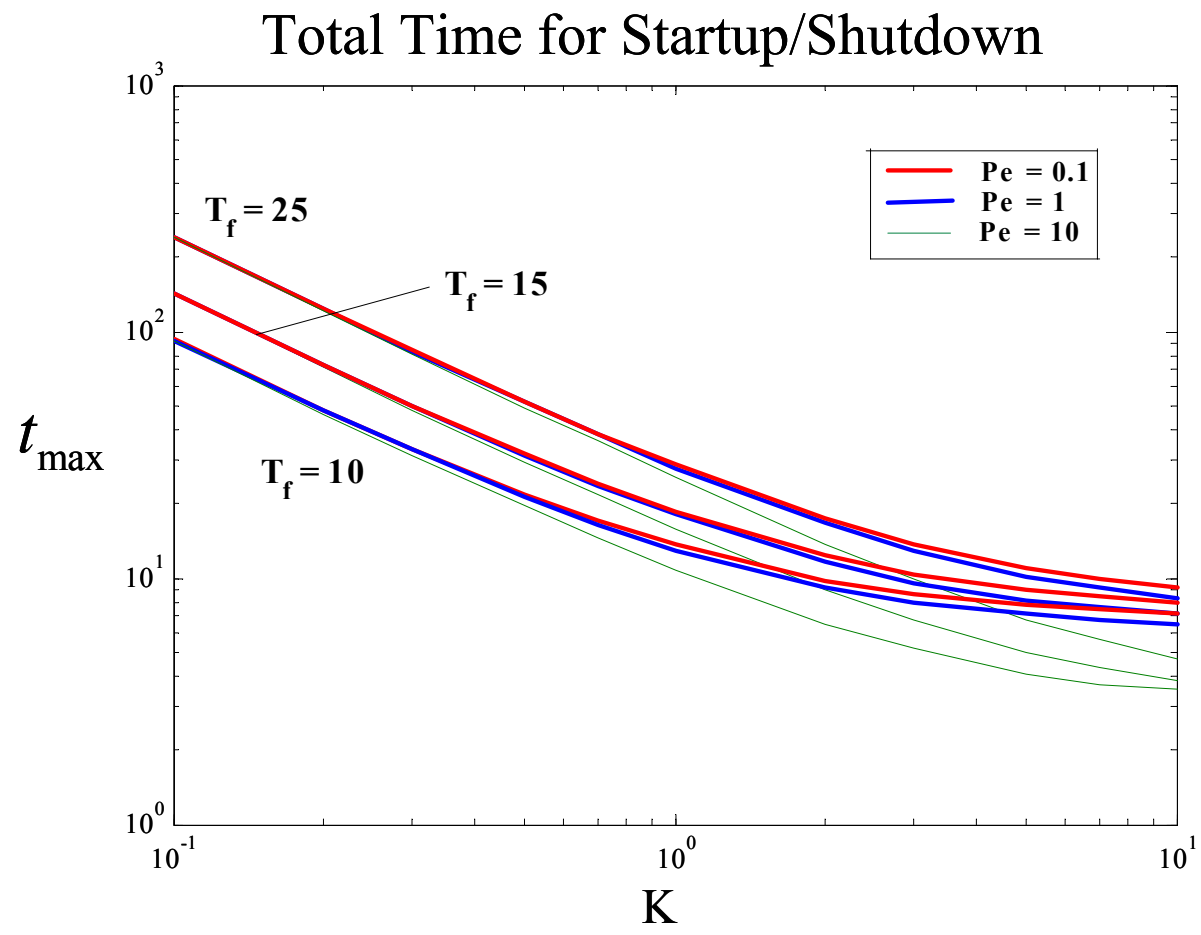

Figure 3-21: Time required to reach operating temperature vs. rate of inlet temperature rise for various final temperatures and Peclet numbers.

\subsubsection{Concluding Remarks}

The second-order convective conductive model has been formulated as the limiting case of the most general, third-order transient thermal model by applying the assumption of local thermal equilibrium in the direction perpendicular to the flow. This allows for a single governing equation to be written for the entire cell, while still accounting for thermal energy storage in the cell materials. The equation is solved in non-dimensional form yielding a closed-form, analytical solution. The information we are most interested in is the maximum temperature gradient developed in the cell, the maximum temperature derivative (time rate of change of temperature) in the cell, and the total time required to reach a specified operating temperature. These results are presented in terms of the Peclet number, dimensionless final temperature, and dimensionless rate of inlet temperature rise.

\subsection{Formulation and Analysis of CFD model}

Several results from detailed 3-dimensional simulations of transient heating of the unit cell are compared to results from the previously developed purely convective $\left(1^{\text {st }}\right.$ model) and convective-conductive $\left(2^{\text {nd }}\right.$ model $)$ models. These results indicate that the simplified analytical models are able to correctly predict general trends and relationships between design variables in the transient process. 
The unit cell is heated from an initial temperature of $298 \mathrm{~K}$ to a final temperature of $898 \mathrm{~K}$ by flowing hot air into the oxidizer channel. The inlet air velocity, $U$, and rate of inlet temperature rise, $K$, are the design variables that influence the total heating time and the spatial and temporal temperature gradients that occur. The 3-D discretized model described in the previously was used here. The flow field in the channel was solved under the assumption of steady, laminar, fully-developed flow, and then this flow field was preserved and used at every time step in calculation of the unsteady temperature field subject to the transient inlet temperature boundary condition.

In the cases that were run, the velocity, $U$, was varied from 1 to $20 \mathrm{~m} / \mathrm{s}$ and the rate of inlet temperature rise, $K$, was varied from 0.01 to $1 \mathrm{~K} / \mathrm{s}$. This is considered a realistic range for these parameters (based on a total heating time of $1 \mathrm{hr}$, and typical air stream velocities found during steady-state operation). The inlet air temperature was increased linearly from $298 \mathrm{~K}$ to $898 \mathrm{~K}$ and then held constant until the normalized temperature of the solid at the exit was within $5 \%$ of its steady-state value.

Figure 3-22-Figure 3-24 show heating time and spatial temperature gradient as a function of $K$ for various inlet velocities. At $U=20 \mathrm{~m} / \mathrm{s}$, the effective Peclet number, $P e$, is high and the Fluent results closely match the predictions of the $1^{\text {st }}$ order analytical model. The $2^{\text {nd }}$ order model predictions are not shown because they are nearly identical to the $1^{\text {st }}$ model. (Remember, the $1^{\text {st }}$ model is the limiting case of the $2^{\text {nd }}$ model as $P e$ grows arbitrarily large.) In Figure $3-23, U=10 \mathrm{~m} / \mathrm{s}$, resulting in $P e=8.355$, and the analytical models again correctly predict heating time and temperature gradient, especially at very low values of $K$. In Figure 3-24, for $U=1 \mathrm{~m} / \mathrm{s}$, the effective Peclet number is less than 1 , and although the analytical models generally predict the correct trends, the error is substantial for large $K$.

Figure 3-25 shows temporal temperature gradient as a function of $K$ for the cases of $U=1$ and 10. At high velocity, both models are accurate, and at low velocity (low $P e$ ) the $2^{\text {nd }}$ model is more accurate as expected.
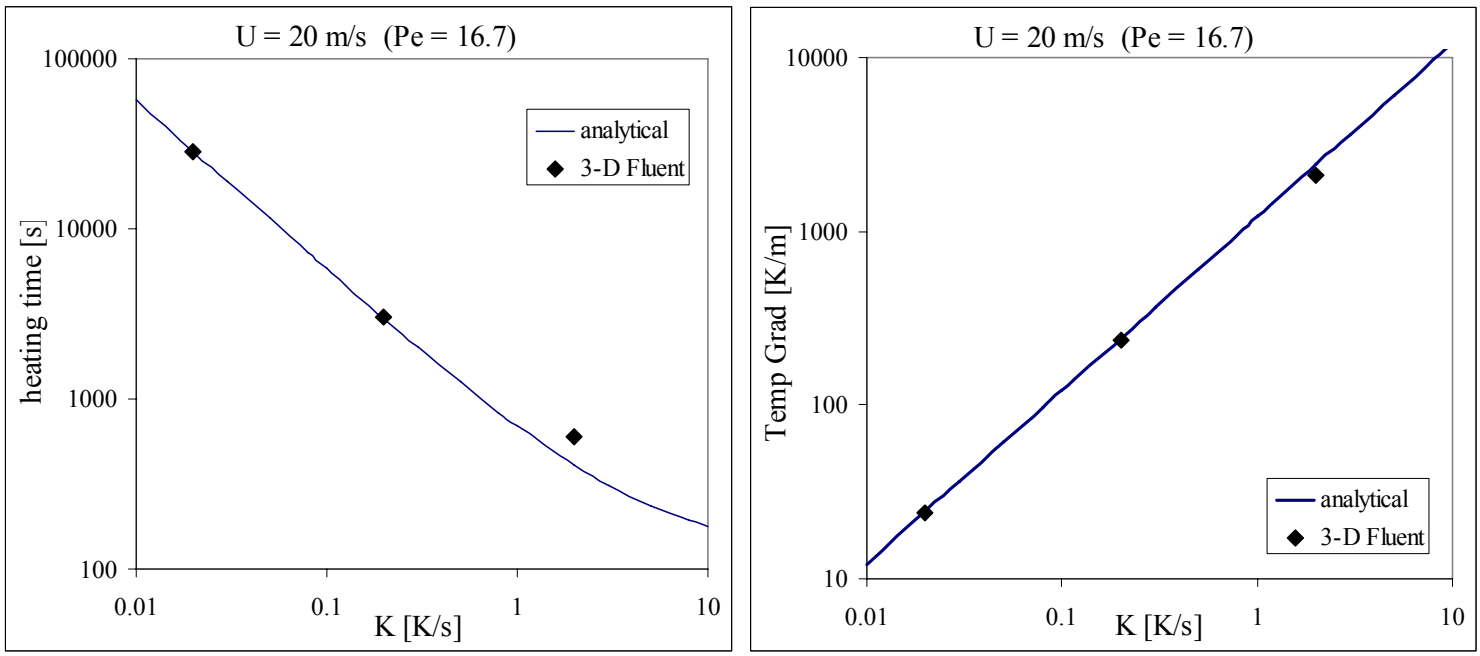
Figure 3-22: Heating time and spatial temperature gradient are shown as a function of rate of inlet temperature rise, $K$. The analytical prediction (solid line) is from the $1^{\text {st }}$ model
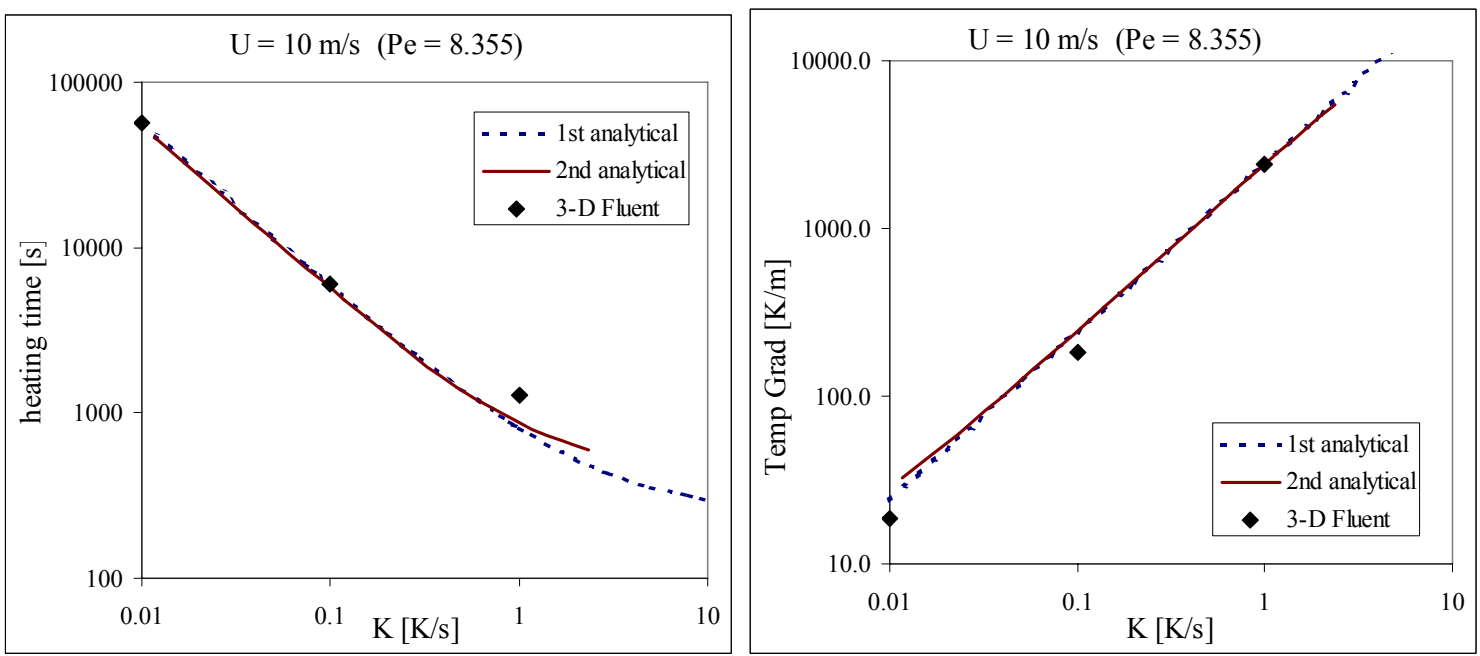

Figure 3-23: Fluent results are compared to the $1^{\text {st }}$ (dotted line) and $2^{\text {nd }}$ (solid line) model predictions of heating time and spatial temperature gradient. The analytical models are accurate within approximately $30 \%$.
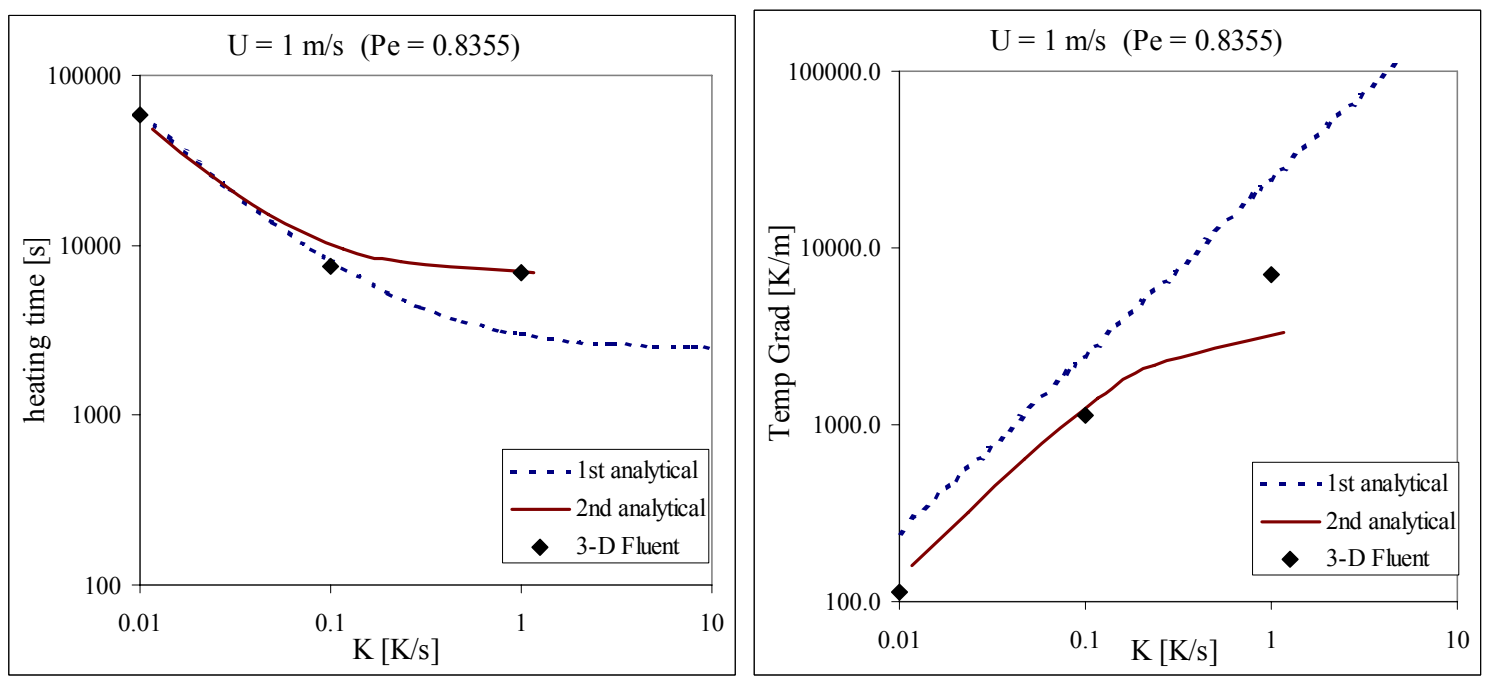

Figure 3-24: Fluent results compared to the $1^{\text {st }}$ (dotted line) and $2^{\text {nd }}$ (solid line) model predictions of heating time and spatial temperature gradient. The $1^{\text {st }}$ model is not expected to be valid for small Peclet number and is shown for reference only. 

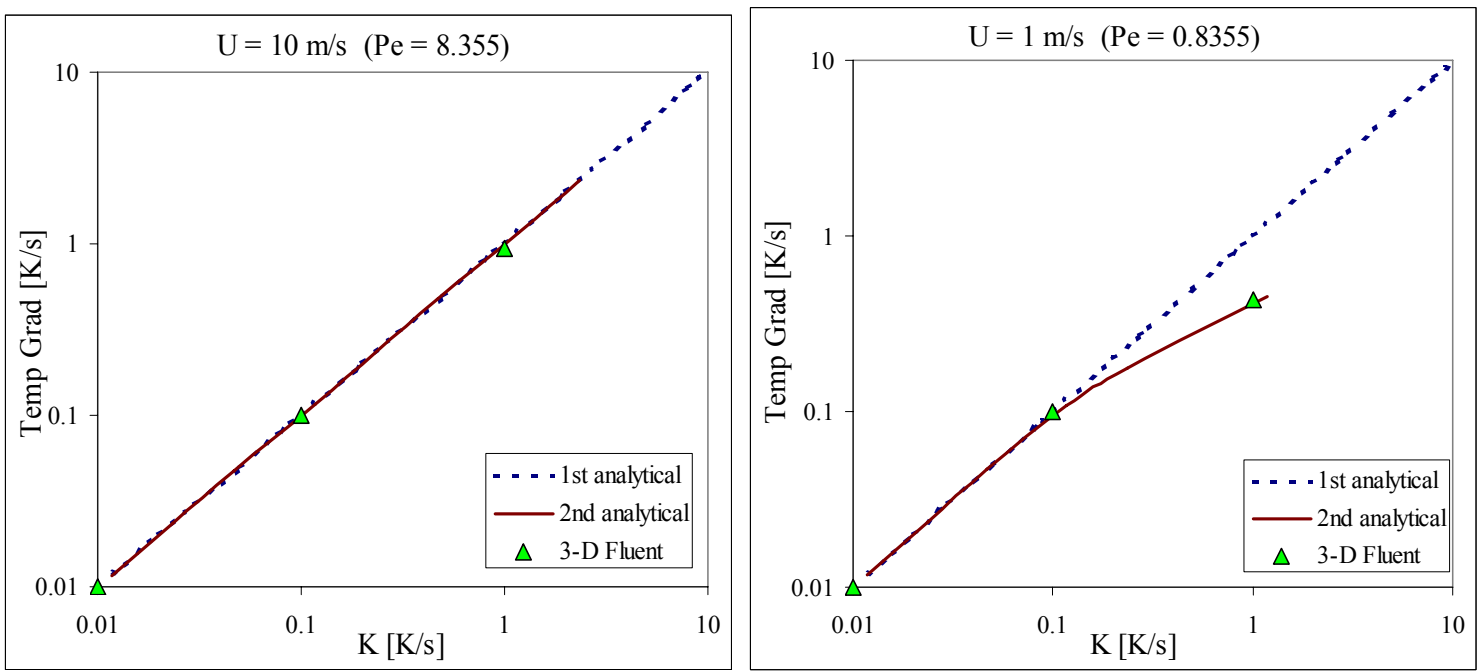

Figure 3-25: Temporal temperature gradient (degrees/second) is plotted vs. rate of inlet temperature rise, $K$, for large and small Peclet number. The analytical model predictions compare favorably to the Fluent results.

A feature common to all of these results is that at low values of $K$, the analytical models are quite accurate for the entire range of $P e$. At very low rates of inlet temperature rise, heating time is almost exclusively determined by the time required for the inlet to reach operating temperature, with the thermal response time of the cell being negligible. Thus, knowledge of the heat transfer mechanisms within the cell is not required in order to predict total heating time. On the other hand, accurate prediction of temperature gradients at very low velocity is best accomplished by the $2^{\text {nd }}$ model, which accounts for diffusion of thermal energy in the solid. From a design point of view it is unfavorable to operate at low velocity due to increased heating time and thermal gradients. For example, with $K=0.1$, the heating time increases by $25 \%$ and maximum temperature gradient increases an order of magnitude when velocity is lowered from 10 to $1 \mathrm{~m} / \mathrm{s}$.

The transient heating of the unit cell has been simulated in FLUENT using a 3-D model. Several cases of various air velocities and rates of inlet temperature rise were compared to predictions of the previously developed analytical models. It is clear that the analytical models capture the overall trends in relationships between parameters, but with somewhat less accuracy than the CFD model. However, the simplicity and lack of computational expense ( $1^{\text {st }}$ order model is a single algebraic equation!) are enormous advantages enjoyed by the analytical models. Thus, it is reasonable that the analytical models could be used to identify an approximate range of operating parameters for a transient process, and then the CFD model could be used as the final design step to precisely pinpoint the ideal operating point. 


\subsubsection{Temperature Gradient Field}

The time-dependent temperature field within the unit cell during transient heating, as found by solution of the CFD model described in April 2005 report, is used to approximately calculate the temperature gradient in the solid PEN structure. Because temperature gradients in the directions normal to flow are minimal compared to the axial direction, they are not included here. The maximum temperature gradients for a given simulation typically occur as the inlet temperature reaches the desired operating temperature. At that point, the inlet temperature is held constant, leading to a gradual decline in gradients as the cell becomes isothermal. This maximum gradient field along the centerline of the cathode is plotted in Figure 3-26 for mean inlet air velocities, U, of 1 and $10 \mathrm{~m} / \mathrm{s}$, and rate of inlet temperature rise, $\mathrm{K}$, of $1{ }^{\circ} \mathrm{C} / \mathrm{s}$. The maximum gradient is significantly reduced as air velocity increases. In Figure $3-27$, the effect of varying $\mathrm{K}$ is shown by fixing $\mathrm{U}=10 \mathrm{~m} / \mathrm{s}$. The temperature gradients are seen to scale directly with $\mathrm{K}$ as predicted by the $1^{\text {st }}$ and $2^{\text {nd }}$ order analytical models. This information is central to the design of an optimal heating or cooling scheme, which minimizes the process time requirements while avoiding excessive, thermally induced stress.

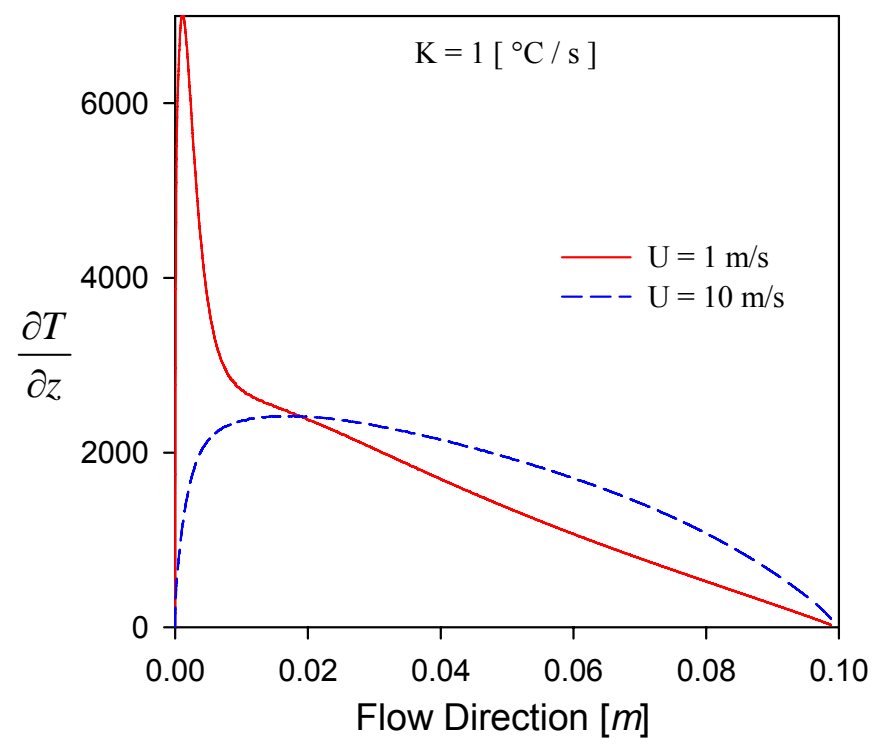

Figure 3-26: Maximum temperature gradient in the axial direction for various inlet air velocities. The rate if inlet temperature rise, $\mathrm{K}$, is $1{ }^{\circ} \mathrm{C} / \mathrm{s}$. 


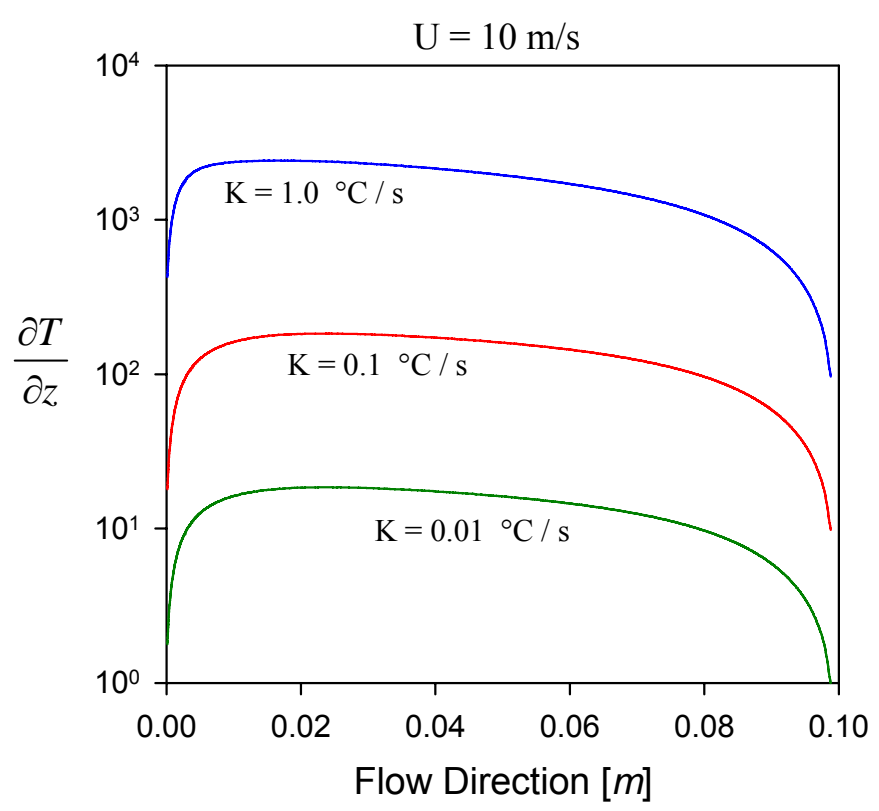

Figure 3-27: Maximum temperature gradient in the axial direction is plotted on a log scale for fixed velocity and various rates of inlet temperature rise.

\subsection{Simplification of $3^{\text {rd }}$ Order Model: 2-eqn coupled solid-gas model}

In addition to validating the simplified analytical models, the results of the CFD model also indicate that lumping the solid equations of the $3^{\text {rd }}$ order numerical model is a reasonable simplifying assumption. Thus, the seven-equation model formulated separately is reduced to a two-equation model - one solid and one gas, coupled through the convective terms:

gas:

$$
\frac{\partial T_{g}}{\partial t}+u \frac{\partial T_{g}}{\partial z}=\alpha_{g} \frac{\partial^{2} T_{g}}{\partial z^{2}}+\frac{h P_{g-s}}{C_{g} A_{c}}\left(T_{s}-T_{g}\right)
$$

solid :

$$
\frac{\partial T_{s}}{\partial t}=\alpha_{s} \frac{\partial^{2} T_{s}}{\partial z^{2}}+\frac{h P_{g-s}}{C_{s} A_{c}}\left(T_{g}-T_{s}\right)
$$

where, the definitions of effective volumetric heat capacity and effective thermal diffusivity are,

$$
C_{g}=\frac{\left(\rho c_{p} A\right)_{g}}{A_{c}} ; \quad C_{s}=\frac{\sum_{i}\left(\rho c_{p} A\right)_{i}}{A_{c}} ; \quad \alpha_{s}=\frac{\sum_{i}(k A)_{i}}{\sum_{i}\left(\rho c_{p} A\right)_{i}} ;
$$

The subscript, $i$, indicates to sum over every solid layer ( 2 interconnects, anode, cathode, electrolyte, and fuel channel). The boundary and initial conditions are, 
gas:

$$
\begin{array}{ll}
\text { B.C.' } s: & T_{g}(0, t)=f(t) ; \quad \frac{\partial T_{g}}{\partial z}(L, t)=0 \\
\text { I.C. }: & T_{g}(z, 0)=T_{o}
\end{array}
$$

solid :

$$
\begin{array}{ll}
\text { B.C.' } s: & \frac{\partial T_{s}}{\partial z}(0, t)=\frac{\partial T_{s}}{\partial z}(L, t)=0 \\
\text { I.C. }: & T_{s}(z, 0)=T_{o}
\end{array}
$$

The discretized gas equation, based on a finite-difference scheme, (with $0 \leq \beta \leq 1$ as a weighting factor between fully implicit and explicit) is,

$$
\begin{aligned}
& -\frac{\beta \Delta t \alpha_{g}}{\Delta x^{2}} T_{i+1}^{n+1}-\frac{\beta \Delta t\left(\alpha_{g}+u\right)}{\Delta x^{2}} T_{i-1}^{n+1}+\left[1+\beta \Delta t\left(\frac{2 \alpha_{g}+u}{\Delta x^{2}}+\frac{h P_{g-s}}{C_{g} A_{c}}\right)\right] T_{i}^{n+1} \\
& =\beta \Delta t \frac{h P_{g-s}}{C_{g} A_{c}} T_{i, s}^{n+1}+T_{i}^{n}+(1-\beta) \Delta t\left[\alpha_{g} \frac{T_{i+1}-2 T_{i}+T_{i-1}}{\Delta x^{2}}+u \frac{T_{i-1}-T_{i}}{\Delta x^{2}}-\frac{h P_{g-s}}{C_{g} A_{c}}\left(T_{i}-T_{i, s}\right)\right]^{n}
\end{aligned}
$$

and the discretized solid equation is,

$$
\begin{aligned}
& -\frac{\beta \Delta t \alpha_{s}}{\Delta x^{2}}\left(T_{i-1}^{n+1}+T_{i+1}^{n+1}\right)+\left[1+\beta \Delta t\left(\frac{2 \alpha_{s}}{\Delta x^{2}}+\frac{h P_{g-s}}{C_{s} A_{c}}\right)\right] T_{i}^{n+1} \\
& =\beta \Delta t \frac{h P_{g-s}}{C_{s} A_{c}} T_{i, g}^{n+1}+T_{i}^{n}+(1-\beta) \Delta t\left[\alpha_{s} \frac{T_{i+1}-2 T_{i}+T_{i-1}}{\Delta x^{2}}+\frac{h P_{g-s}}{C_{s} A_{c}}\left(T_{i, g}-T_{i}\right)\right]^{n}
\end{aligned}
$$

These equations are more easily written in matrix form,

gas :

$$
\left[\mathbf{C}_{1}\right] \mathbf{T}_{g}^{n+1}=\lambda_{1} \mathbf{T}_{s}^{n+1}+\left[\mathbf{C}_{2}\right] \mathbf{T}_{g}^{n}+\lambda_{2} \mathbf{T}_{s}^{n}
$$

solid :

$$
\left[\mathbf{C}_{3}\right] \mathbf{T}_{s}^{n+1}=\lambda_{3} \mathbf{T}_{g}^{n+1}+\left[\mathbf{C}_{4}\right] \mathbf{T}_{s}^{n}+\lambda_{4} \mathbf{T}_{g}^{n}
$$

where, $\mathbf{C}_{1}, \mathbf{C}_{2}, \mathbf{C}_{3}, \mathbf{C}_{4}$ are coefficient matrices, and the constants are, 


$$
\begin{aligned}
& \lambda_{1}=\Delta t \frac{\beta h P_{g-s}}{C_{g} A_{c}} ; \quad \lambda_{2}=-\Delta t \frac{(1-\beta) h P_{g-s}}{C_{g} A_{c}} \\
& \lambda_{3}=\Delta t \frac{\beta h P_{g-s}}{C_{s} A_{c}} ; \quad \lambda_{4}=-\Delta t \frac{(1-\beta) h P_{g-s}}{C_{s} A_{c}}
\end{aligned}
$$

The solid-gas heat transfer coefficient, $h$, is not well-known, but as a first approximation, the Nusselt number for laminar flow with constant wall temperature will be used,

$$
N u_{D}=3.66=\frac{h D}{k} \rightarrow h \approx 61 \quad\left[W m^{-2} K^{-1}\right]
$$

Fortunately, the matrices are tri-diagonal allowing use of the TDMA algorithm for efficient solution. The computer code for this has already been developed by Press, et al. ${ }^{5}$, and their subroutine "tridag" written in the C language has been obtained and implemented. The procedure for solving the equations is as follows:

1. populate the coefficient matrices

2. guess the temperature in the solid for the future time step

3. calculate the rhs of the gas equation

4. calculate the future temperature in the gas using "tridag"

5. using the results of the previous step (4), calculate the rhs of the solid equation

6. calculate the future temperature in the solid using "tridag"

7. compare the results of the previous step (6) to the guess used in step (2).

8. Repeat steps (2) - (7) using the calculated solid temperature as the new guess in step (2) until convergence is obtained.

9. Proceed to the next time step and return to step (1).

The best initial guess for step (2) is probably the solid temperature at the current time step.

Maximum temperature gradient fields developed during transient heating of the unit cell have been presented. These are of vital importance in predicting stress fields and failure probability of the solid components. CFD results indicate that temperature gradients normal to the axial direction are minimal. Thus, the $3^{\text {rd }}$ order transient model has been reduced from seven equations to a 2-equation, coupled solid-gas model. A computer code to numerically solve this model is under development.

\subsubsection{Validation 2-eqn coupled solid-gas model}

Following the development of the two-equation solid-gas model a finite difference code has been developed and used to obtain preliminary results. Figure 3-28 and Figure 3-29 show the development of the temperature field in the channel walls

\footnotetext{
${ }^{5}$ Press, W.H., Teukolsky, S.A., Vetterling, W.T., Flannery, B.P., (1992), Numerical Recipes in C, $2^{\text {nd }}$ ed., Cambridge University Press, pp. 50-51.
} 
during the initial stages of heating and during the final stages as the steady state temperature is approached. The heating air velocity used for this simulation is $5 \mathrm{~m} / \mathrm{s}$ and the rate of inlet temperature rise is $0.5^{\circ} \mathrm{C} / \mathrm{s}$. These representative plots demonstrate the capability of the code, which has not yet been validated.

Validation of the code will include several tasks. First, a mesh and time step analysis will insure the results are mesh and time-step independent. Second, the model will be validated against the analytical solution provided by the $2^{\text {nd }}$ order, local thermal equilibrium model. As the heat transfer coefficient coupling the solid and gas equations becomes arbitrarily large, the temperature difference between the two equations vanishes and the solution should converge on the analytical model. Finally, temperature profiles will be compared to the results of highly detailed Fluent simulations to determine the range of applicability and validity of the $3^{\text {rd }}$ order model.

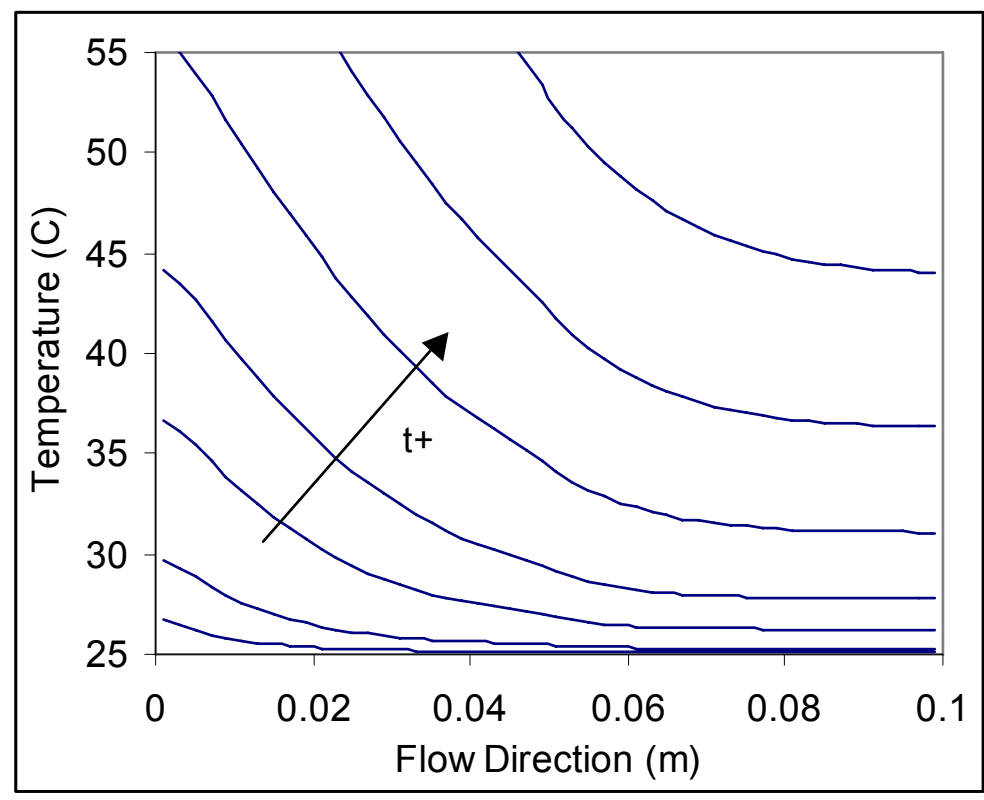

Figure 3-28: Time-varying temperature profiles in the unit cell during the initial stages of a heating process. 


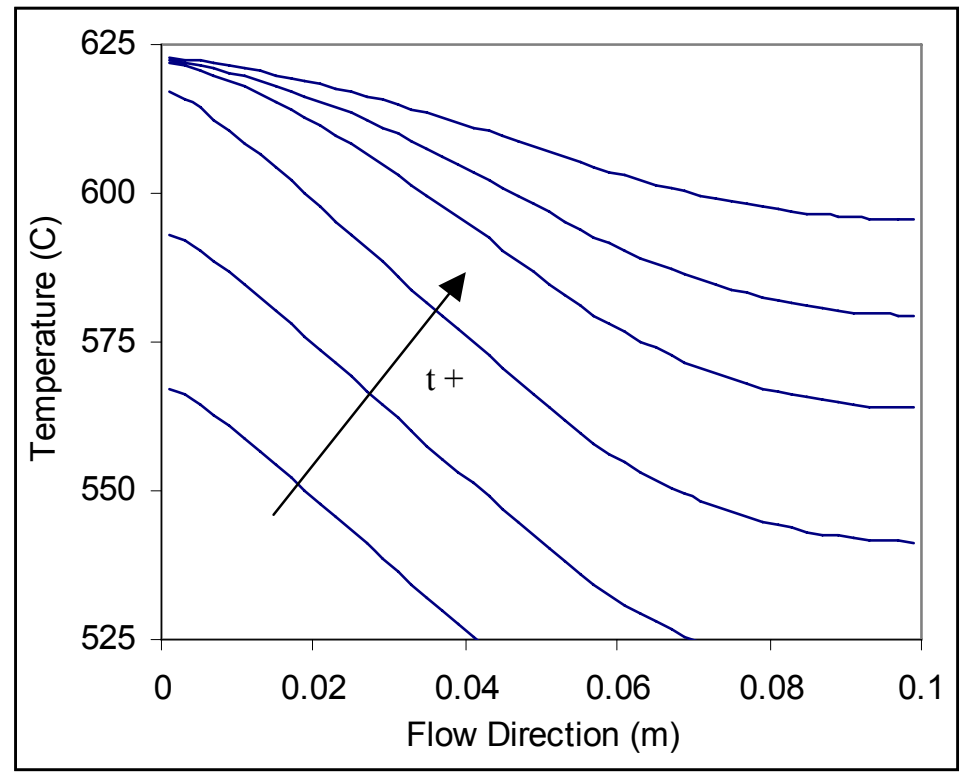

Figure 3-29: Time-varying temperature profiles during the latter stages of a heating process as the cell approaches steady-state operating temperature.

\subsubsection{Concluding Remarks}

A computer code to numerically solve the $3^{\text {rd }}$ order transient coupled solid-gas model has been developed. After validation of the code, the model results will be analyzed and compared to the $1^{\text {st }}$ and $2^{\text {nd }}$ order analytical models, and 3-D Fluent results.

\subsubsection{Two-Equation Coupled Solid-Gas Model}

After validation of the $3^{\text {rd }}$ order coupled heating model code, we present model results for a range of key operating parameters - heating air velocity, $\mathrm{U}$, and heating rate, $\mathrm{K}$. In an optimized transient heating process, the heating time is minimized under the constraint of maximum allowable temperature gradients in the solid. Thus, these quantities are calculated and presented in Figure 3-30 through Figure 3-32 for $U=1,10$, and $20 \mathrm{~m} / \mathrm{s}$, and various $\mathrm{K}$. In addition, the analytical model and 3-D Fluent results are included for a side-by-side comparison.

Heating times predicted by all models are in good agreement, especially at low values of $\mathrm{K}$. The agreement is better, over a larger range of $\mathrm{K}$, as velocity increases. However, maximum temperature gradient developed during the heating process is generally over- predicted by the 3rd order numerical model. Closer investigation has indicated that this is a strong function of a specific empirical correlation for heat transfer coefficient, $h$, that is required for coupling the solid and gas equations. The correlation currently being used, over-estimates the rate at which $h$ increases near the inlet compared to its thermally-fully developed value downstream. In reality, $h$ varies with air velocity, distance from the inlet, and time. This relationship is not known explicitly, requiring solution of a much more complicated conjugate heat transfer problem. Adding this level of complexity to the 2-eqn model would cut into its advantage of being much simpler and more computationally efficient than the Fluent model. And because the $3^{\text {rd }}$ order, 2-eqn 
numerical model shows little or no improvement in accuracy over the simpler analytical models which we have developed earlier, its utility is severely limited.
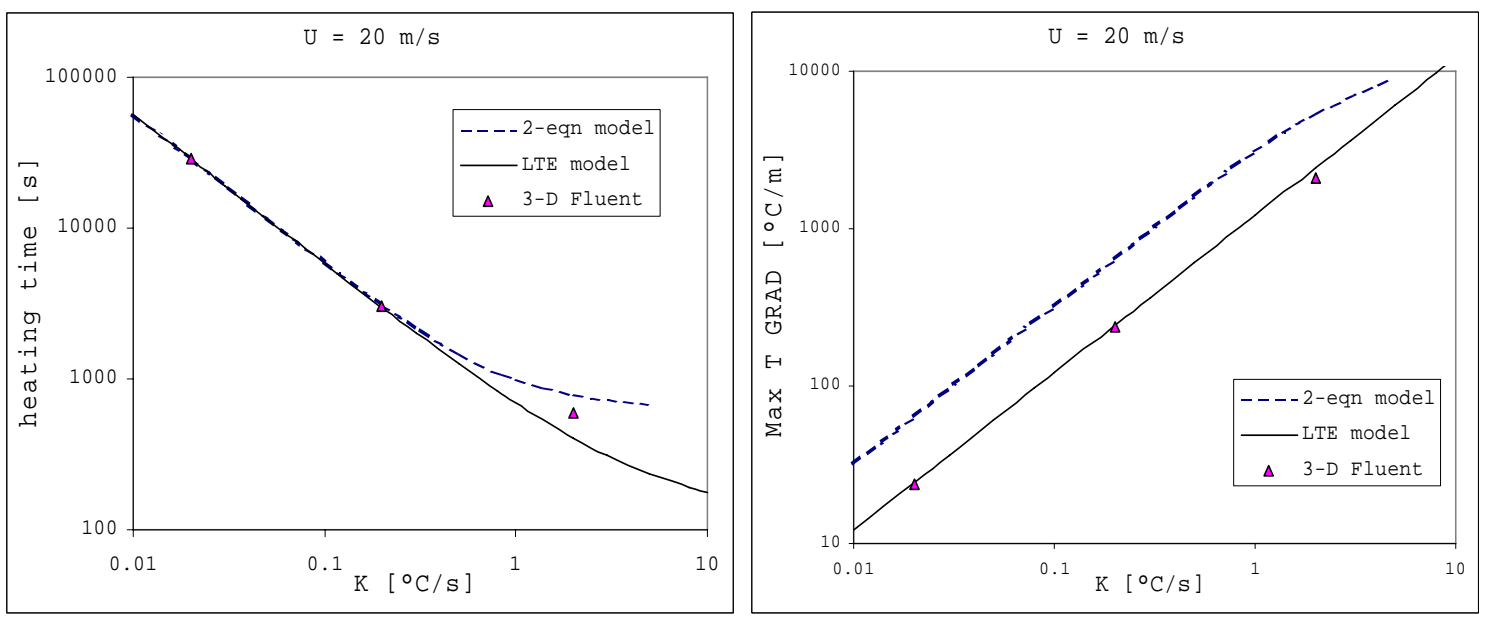

Figure 3-30: Heating time and maximum temperature gradient for various heating rate, $\mathrm{K}$, and fixed hot air velocity, $\mathrm{U}=20 \mathrm{~m} / \mathrm{s}$. The $3^{\text {rd }}$ order, 2-eqn model (dashed line) is compared to the $1^{\text {st }}$ order LTE analytical model (solid line) and 3-D Fluent (triangle) results.
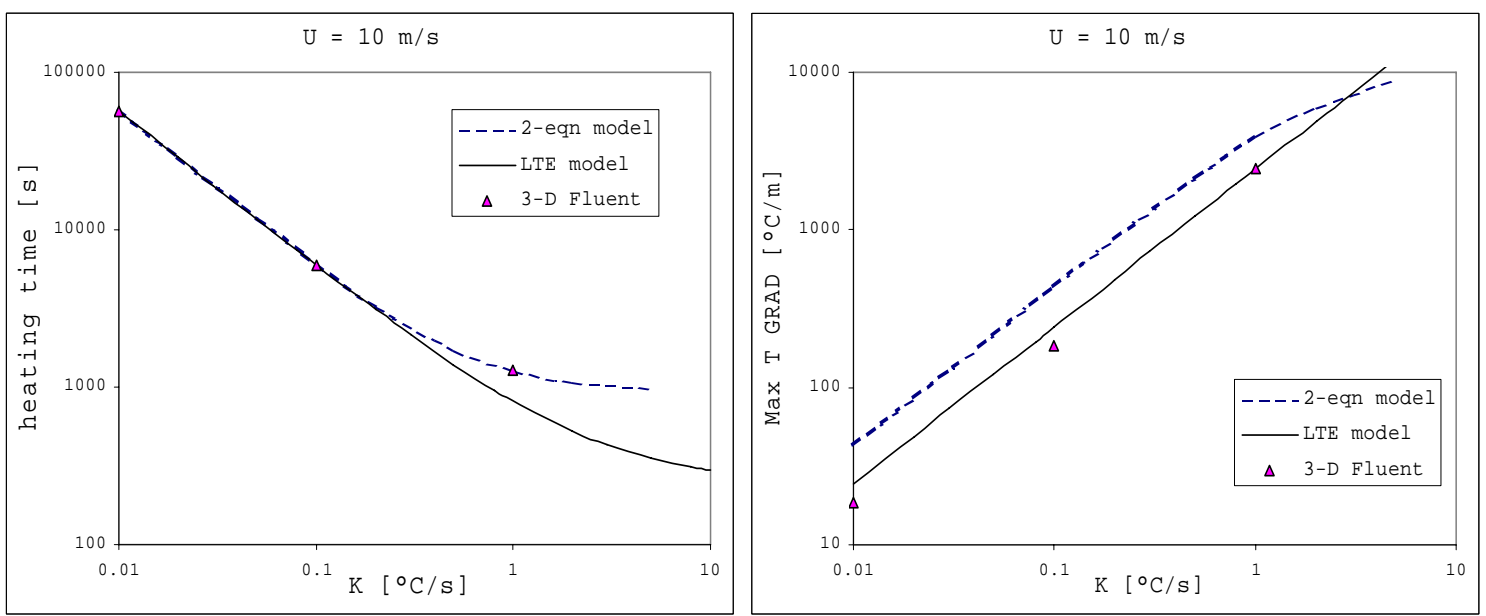

Figure 3-31: Heating time and maximum temperature gradient for hot air velocity, $\mathrm{U}=$ $10 \mathrm{~m} / \mathrm{s}$. 

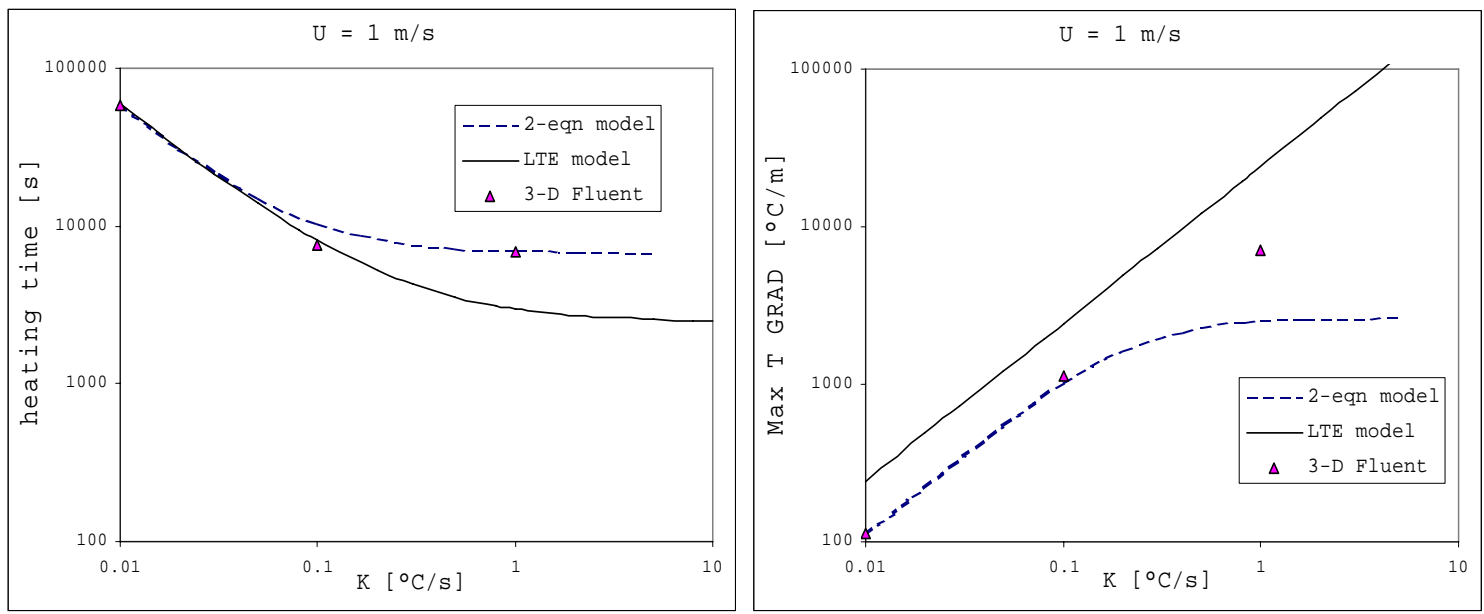

Figure 3-32: Heating time and maximum temperature gradient for hot air velocity, $U=1$ $\mathrm{m} / \mathrm{s}$.

\subsubsection{Concluding Remarks}

Results of the $3^{\text {rd }}$ order, 2-eqn, non-equilibrium model for transient heating/cooling of the cell have been presented, and a direct comparison to the analytical and CFD models has been made. The 2-eqn model, while significantly simpler and less computationally expensive than the CFD model, does not show a sufficiently significant improvement in accuracy to warrant its use over the analytical models that allow closedform solution of the problem. The primary source of limited success of the $3^{\text {rd }}$ order model is in the strong dependence of predicted maximum temperature gradient on the heat transfer coefficient, $h$, coupling the solid and gas equations. In the future, a complete objective comparison of the analytical models, the $3^{\text {rd }}$ order, 2-eqn numerical model, and the Fluent model will allow us to 1) identify the model that is best suited for industry use and 2) recommend the direction of future research in this area.

\subsection{Evaluation of transient thermal models}

After reviewing the results and analysis of the simplified analytical transient heating models (April 2005 report) and the numerical, 2-eqn, non-equilibrium model (August 2005), we now provide guidance concerning model selection and limits of applicability. Thermal modeling efforts thus far have focused on correlating the heating rate and the observed temperature gradients in a unit cell heated by flowing hot air into the oxidizer channel. The linear rate of temperature rise, $K$, and mean velocity, $U$, of the heating air are design variables that can be controlled in maintaining temperature gradients below a given threshold while minimizing heating time requirements. In the analysis of each simplified model, the predictions of heating time and maximum temperature gradient developed in the cell were compared to results from 3-D, CFD Fluent model simulations. Thus, we assessed the capability of these models to predict these global quantities while making no judgment concerning the ability of the models to accurately predict detailed time-varying temperature fields. 
Since the $1^{\text {st }}$ order transient heating model (in the thermally thin limit) yields simple algebraic relations between temperature gradient, heating time, heating rate, and air velocity, we establish its range of validity and then investigate the $2^{\text {nd }}$ and $3^{\text {rd }}$ order models to determine if they provide additional improvements over this baseline case. Early on, it was discovered that the air velocity, $U$, in the $1^{\text {st }}$ order model should be replaced by effective velocity, defined as,

$$
u_{\text {eff }}=\frac{\left(\rho c_{p} A\right)_{g}}{\sum_{i}\left(\rho c_{p} A\right)_{i}} \cdot U
$$

where the summation in the denominator is over every component of the cell, and $\rho, c_{p}$, and $A$, are the density, specific heat, and cross-sectional area, respectively of each material. Thus, effective velocity is the real velocity scaled by the ratio of heat capacity in the air stream to thermal energy storage in the walls. The model predictions for heating time, spatial temperature gradient, and temporal temperature gradient are,

$$
\tau_{h}=\frac{T_{f}-T_{o}}{K}+\frac{L}{u_{e f f}} ; \quad\left(\frac{\partial T}{\partial z}\right)_{\max }=\frac{K}{u_{e f f}} ; \quad\left(\frac{\partial T}{\partial t}\right)_{\max }=K ;
$$

where $T_{f}$ and $T_{o}$ are the final steady state temperature and initial temperature, respectively. The range of values of $U$ and $K$ for which Eqs. (3.61)-(3.62) are valid is shown in Figure 3-33-Figure 3-35. The model predicts heating time and temporal temperature gradient to a high degree of accuracy ( $\sim 2 \%$ error) compared to CFD results. Model predictions of maximum spatial temperature gradient (along the flow direction) are less precise $\left(<20 \%\right.$ error). For the values of $\mathrm{K}$ and $\mathrm{U}$ given, the $1^{\text {st }}$ and $2^{\text {nd }}$ order models yield almost identical results and outside this range the $2^{\text {nd }}$ order model does not improve accuracy except in the case of predicting temperature gradients at very low velocity $\left(<1 \mathrm{~m} / \mathrm{s}\right.$ ) and $\mathrm{K}<0.1{ }^{\circ} \mathrm{C} / \mathrm{s}$ (see Figure 3-34). The $3^{\text {rd }}$ order, 2-eqn model is also not an improvement over the $1^{\text {st }}$ model, and, in fact, has limited ability to predict temperature gradients due to its strong dependence on the solid-gas heat transfer coefficient in the air channel. 


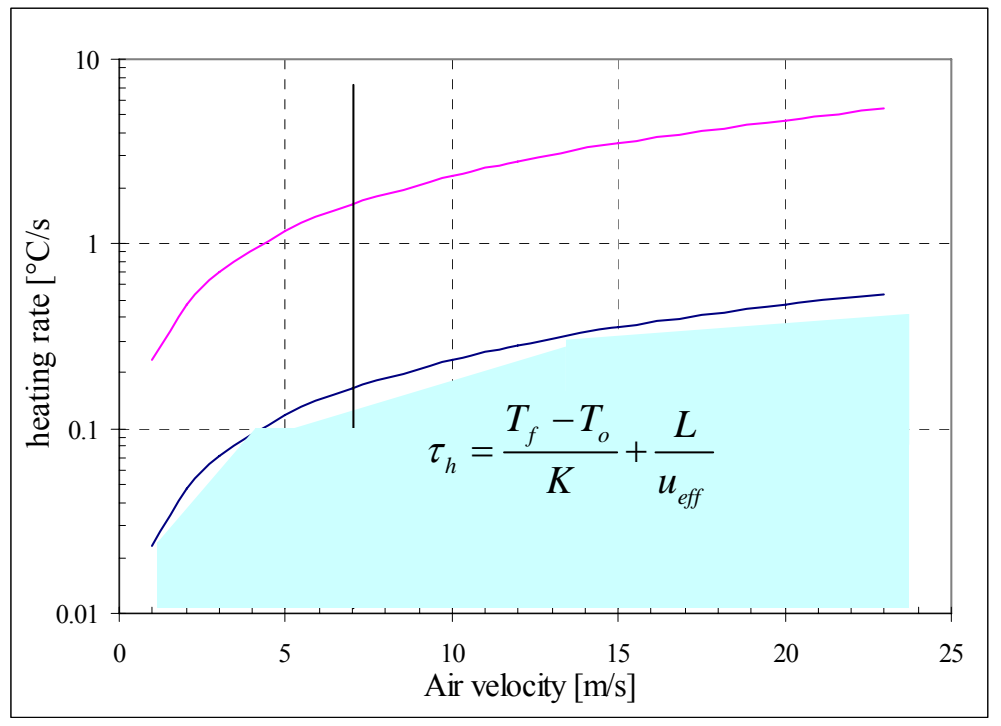

Figure 3-33: The range of values (lightly shaded area) of heating rate, $K$, and mean air velocity, $U$, for which the $1^{\text {st }}$ order heating model predicts heating time accurate to within $\sim 2 \%$ of CFD predictions.

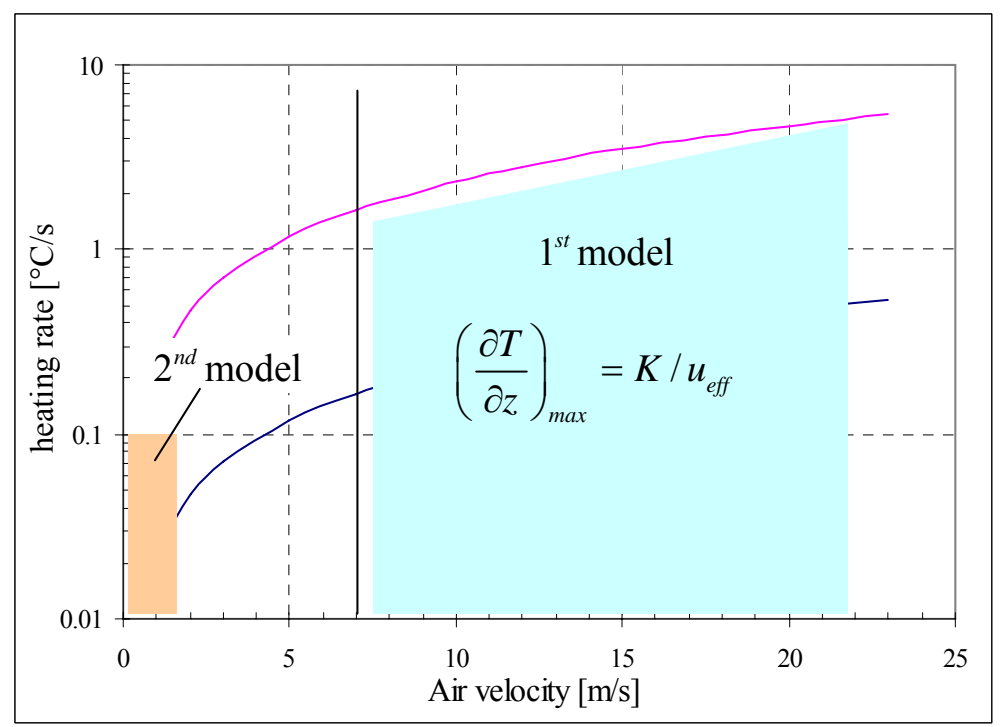

Figure 3-34: Range of values of heating rate, $\mathrm{K}$, and mean air velocity, $\mathrm{U}$, for which the $1^{\text {st }}$ and $2^{\text {nd }}$ order heating models predicts maximum temperature gradient to within $\sim 20 \%$ of CFD predictions. 


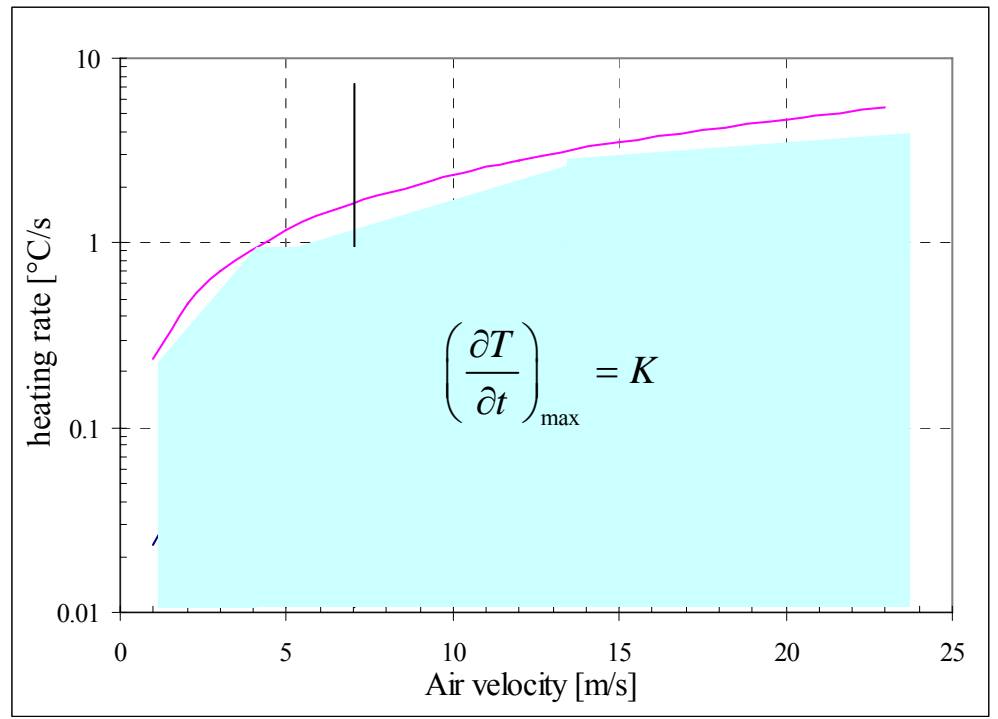

Figure 3-35: The range of values of heating rate, $\mathrm{K}$, and mean air velocity, $U$, for which the $1^{\text {st }}$ order heating model predicts maximum temporal temperature gradient accurate to within $\sim 2 \%$ of CFD predictions.

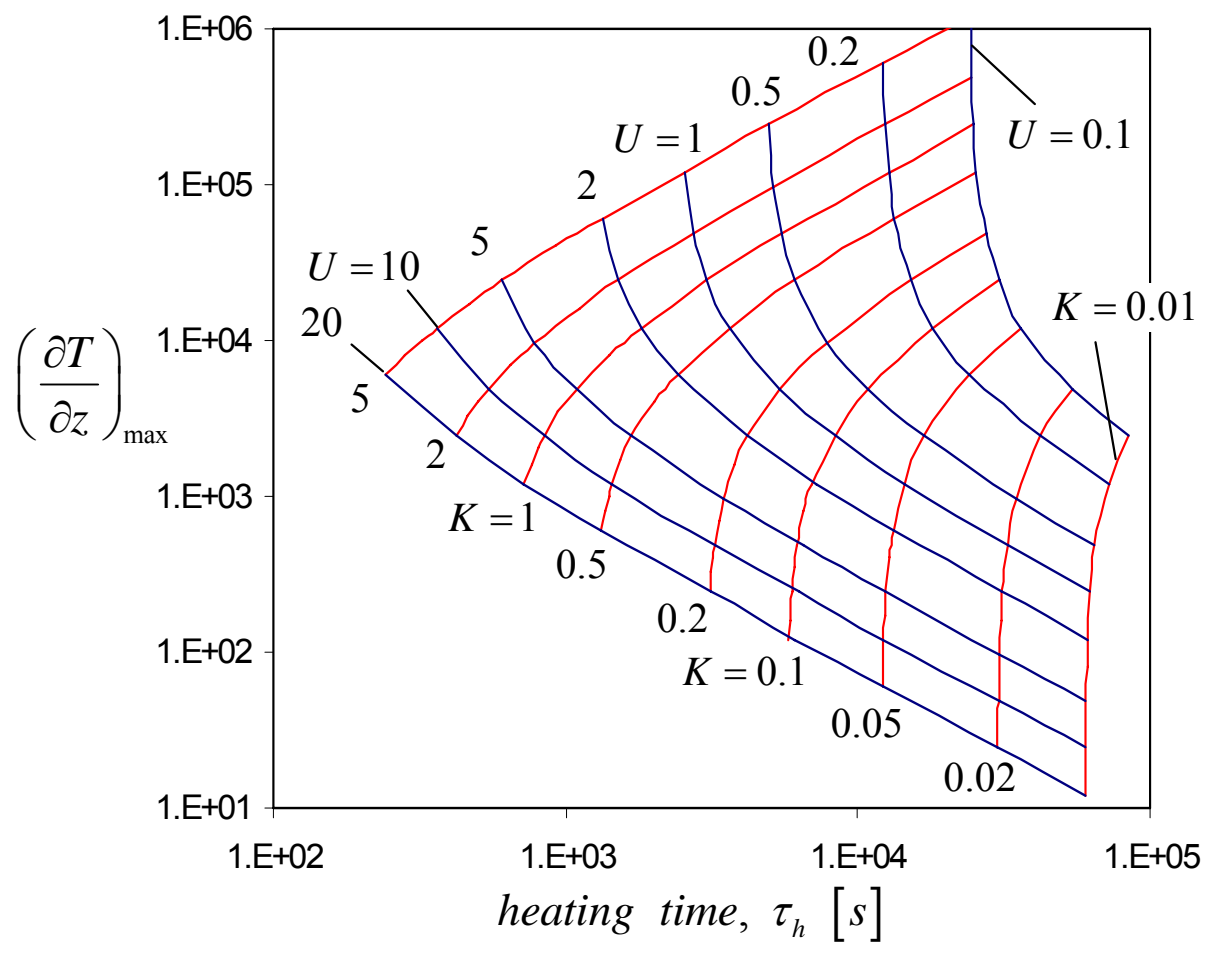

Figure 3-36: Design map showing maximum temperature gradient vs. heating time for various values of $K$ and $U$. Given the maximum allowable temperature gradient, $K$ and $U$ can be selected such that heating time is minimized. This map is specific to the unit cell geometry and materials under consideration, and a temperature rise of $600{ }^{\circ} \mathrm{C}$ from ambient to operating temperature. 
Equations (3.62) provide simple, concise design rules for optimizing a heating/cooling process. Figure 3-36 is a design map, based on these equations, for the specific unit cell geometry and materials described in the March 2004 report. One way to use the map is to draw a horizontal line corresponding to the maximum allowable temperature gradient. Values of $K$ and $U$ below this line can be selected such that the heating time (horizontal axis) is minimized. This gives some flexibility in choosing $K$ and $U$ but these parameters may be limited by other system considerations such as pumping power, or heater size. Alternatively, the heating time requirement could be imposed, and then $K$ and $U$ selected such that temperature gradient is minimized. Use of the map in this way gives approximate values of $K$ and $U$, which are an excellent starting point for performing CFD simulations of the heating/cooling process if detailed results are required.

\subsubsection{Concluding Remarks}

The simplified transient heating models that were developed have been evaluated based on accuracy, simplicity, and computational expense. The simplest $1^{\text {st }}$ order model performs remarkably well for a range of heating rates and hot air velocities that is believed to be relevant to SOFC operation. This model yielded simple algebraic relationships between heating time, temperature gradient, heating rate, and air velocity. The $2^{\text {nd }}$ and $3^{\text {rd }}$ order models did little to improve beyond the $1^{\text {st }}$ order model in terms of accuracy or range of validity, and are significantly more complex and add a small amount of computational expense. If details of the 3-dimensional time-varying temperature fields are desired, then transient CFD modeling is required (at significant computational expense). The $1^{\text {st }}$ order model may safely be used within the range of validity that was established and for the purpose of predicting total heating time, and maximum temperature gradients developed during a heating/cooling process.

\section{REFERENCES}

1. Khaleel, M.A., et al., A finite element analysis modeling tool for solid oxide fuel cell development: coupled electrochemisty, thermal, and flow analysis in MARC. Journal of Power Sources, 2004. 130: p. 136-148.

2. Yakabe, H., et al., 3-D model calculation for planar SOFC. Journal of Power Sources, 2001. 102: p. 144-154.

3. Johnson, J., Fracture failure of solid oxide fuel cells, in Mechanical Engineering. 2004, Georgia Institute of Technology: Atlanta. p. 114.

4. Anderson, T.L., Fracture Mechanics: Fundamentals and Applications. 2 ed. 1995: CRC Press.

5. Rice, J.R., Z. Suo, and J.-S. Wang, Mechanics and thermodynamics of brittle interfacial failure in bimaterial systems, in Metal-Ceramic Interfaces, M. Ruhle, et al., Editors. 1990, Pergamon Press: New York. p. 269-294.

6. Hutchinson, J.W. and Z. Suo, Mixed Mode Cracking in Layered Materials. Advances in Applied Mechanics, 1991. 29: p. 64-187. 
7. Nakamura, T., Three-dimensional stress fields of elastic interface cracks.

Transactions of the ASME. Journal of Applied Mechanics, 1991. 58(4): p. 939-

46.

8. Rice, J.R., A path independent integral and the approximate analysis of strain concentration by notches and cracks. Journal of Applied Mechanics, 1968. 35: p. 379-386.

9. Li, F.Z., C.F. Shih, and A. Needleman, A comparison of methods for calculating energy release rates. Engineering Fracture Mechanics, 1985. 21(2): p. 405-21.

10. Nahta, R. and B. Moran, Domain integrals for axisymmetric interface crack problems. International Journal of Solids and Structures, 1993. 30(15): p. 202740.

11. Shih, C.F. and R.J. Asaro, Elastic-Plastic Analysis of Cracks on Bimaterial Interfaces .1. Small-Scale Yielding. Journal of Applied Mechanics-Transactions of the Asme, 1988. 55(2): p. 299-316.

12. Gosz, M. and B. Moran, An interaction energy integral method for computation of mixed-mode stress intensity factors along non-planer crack fronts in three dimensions. Engineering Fracture Mechanics, 2002. 69: p. 299-319.

13. Kassir, M.K. and A.M. Bregman, Stress-Intensity Factor for a Penny-Shaped Crack between Two Dissimilar Materials. Journal of Applied Mechanics, 1972. 39(1): p. 308-\&.

14. Tada, H., P.C. Paris, and G.R. Irwin, The Stress Analysis of Cracks Handbook. 3rd Edition ed. 2000, New York: ASME Press.

15. Wilson, W.K. and I.W. Yu, Use of the J-Integral in Thermal-Stress Crack Problems. International Journal of Fracture, 1979. 15(4): p. 377-387. 


\section{Appendix A: Thermal Residue Stress Induced Deformation in Functionally Graded Multilayers}

Consider a multilayered laminate of $2 a \times 2 b$, as shown in Fig. A.1. The cross-section of the laminate is shown in schematically shown in Fig. A.2. Each the thermomechanical properties of each layer may very through its thickness.

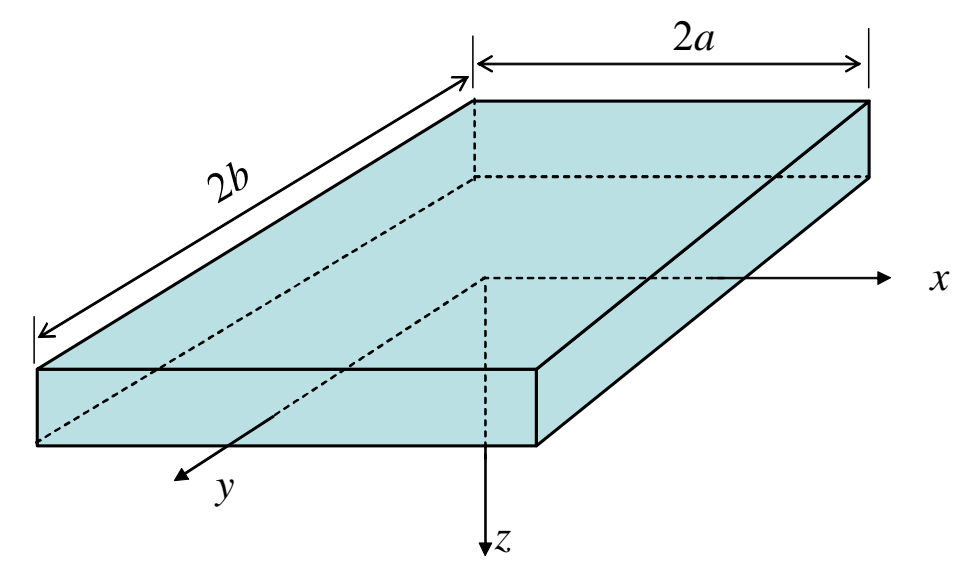

Fig. A.1 A functionally graded multiple layer laminate

\section{Lamina Number}

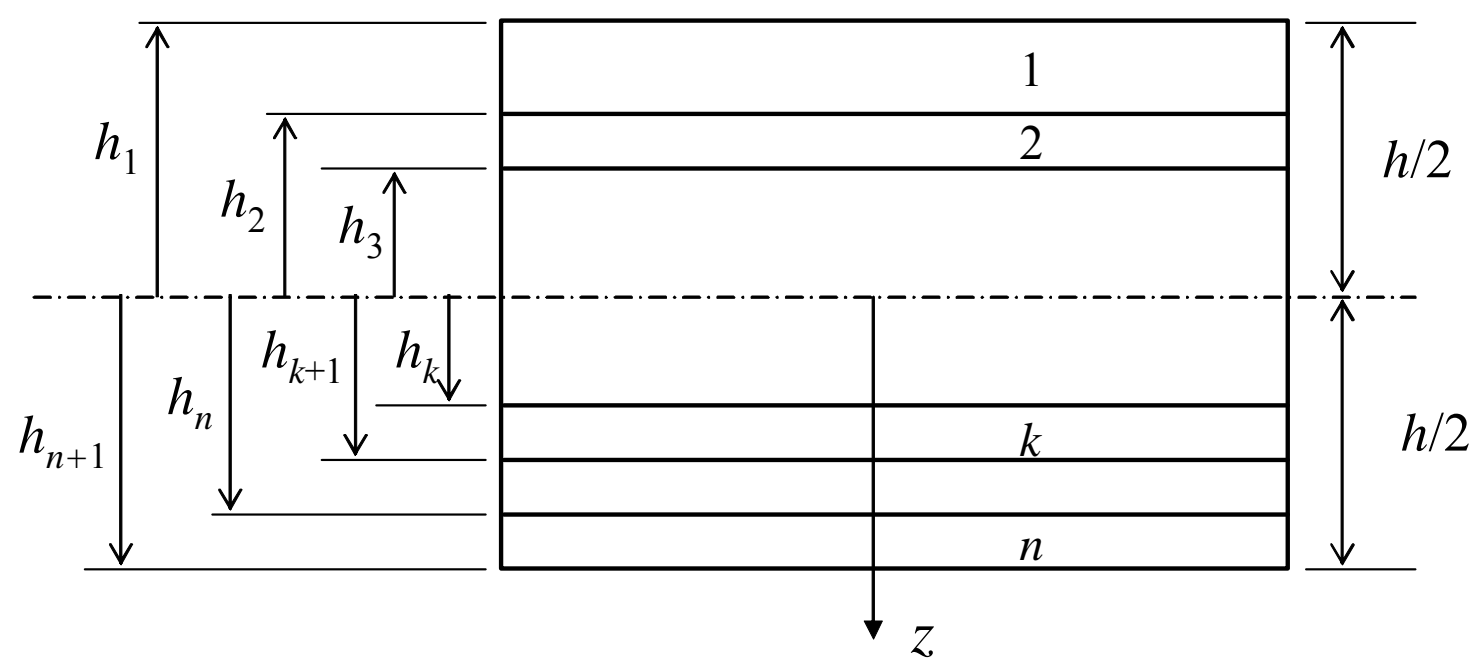

Fig. A.2 Cross section of a multilayer laminate

Let $u, v$ and $w$ be the displacement in the $\mathrm{x}$-direction, $\mathrm{y}$-direction and z-direction, respectively. Then, the strain in the laminate can be written as

$$
\varepsilon_{x}=\frac{\partial u}{\partial x}=\frac{\partial u_{0}}{\partial x}-z \frac{\partial^{2} w_{0}}{\partial x^{2}}
$$




$$
\begin{aligned}
& \varepsilon_{y}=\frac{\partial v}{\partial y}=\frac{\partial v_{0}}{\partial y}-z \frac{\partial^{2} w_{0}}{\partial y^{2}} \\
& \gamma_{x y}=\frac{\partial u}{\partial y}+\frac{\partial v}{\partial x}=\frac{\partial u_{0}}{\partial y}+\frac{\partial v_{0}}{\partial x}-2 z \frac{\partial^{2} w_{0}}{\partial x \partial y}
\end{aligned}
$$

where $u_{0}, v_{0}$ and $w_{0}$ are the displacements of the middle plane $z=0$. The preceding strain-displacement relationship can be written in terms of the midplane strains and the plate curvature as follows:

$$
\left[\begin{array}{c}
\varepsilon_{x} \\
\varepsilon_{y} \\
\gamma_{x y}
\end{array}\right]=\left[\begin{array}{c}
\varepsilon_{x}^{0} \\
\varepsilon_{y}^{0} \\
\gamma_{x y}^{0}
\end{array}\right]+z\left[\begin{array}{c}
\kappa_{x} \\
\kappa_{y} \\
\kappa_{x y}
\end{array}\right]
$$

where

$$
\left[\begin{array}{c}
\varepsilon_{x}^{0} \\
\varepsilon_{y}^{0} \\
\gamma_{x y}^{0}
\end{array}\right]=\left[\begin{array}{c}
\partial u_{0} / \partial x \\
\partial v_{0} / \partial y \\
\partial u_{0} / \partial y+\partial v_{0} / \partial x
\end{array}\right],\left[\begin{array}{c}
\kappa_{x} \\
\kappa_{y} \\
\kappa_{x y}
\end{array}\right]=-\left[\begin{array}{c}
\partial^{2} w_{0} / \partial x^{2} \\
\partial^{2} w_{0} / \partial y^{2} \\
2 \partial^{2} w_{0} / \partial x \partial y
\end{array}\right]
$$

Assuming each layer is isotropic, linearly elastic. Then, Hooke's law yields the stresses in each layer

$$
\left[\begin{array}{c}
\sigma_{x} \\
\sigma_{y} \\
\tau_{x y}
\end{array}\right]=\left[\begin{array}{ccc}
Q_{11} & Q_{12} & 0 \\
Q_{12} & Q_{11} & 0 \\
0 & 0 & Q_{33}
\end{array}\right]\left\{\left[\begin{array}{c}
\varepsilon_{x} \\
\varepsilon_{y} \\
\gamma_{x y}
\end{array}\right]-\alpha \Delta T\left[\begin{array}{l}
1 \\
1 \\
0
\end{array}\right]\right\}
$$

where $\alpha$ is the coefficient of thermal expansion (CTE) and $\Delta T$ is the temperature change. The above can be recast into the following form,

$$
\left[\begin{array}{c}
\sigma_{x} \\
\sigma_{y} \\
\tau_{x y}
\end{array}\right]=\mathbf{Q}\left[\begin{array}{c}
\varepsilon_{x}^{0} \\
\varepsilon_{y}^{0} \\
\gamma_{x y}^{0}
\end{array}\right]+z \mathbf{Q}\left[\begin{array}{c}
\kappa_{x} \\
\kappa_{y} \\
\kappa_{x y}
\end{array}\right]-\alpha \Delta T \mathbf{Q}\left[\begin{array}{l}
1 \\
1 \\
0
\end{array}\right]
$$

where

$$
\mathbf{Q}=\left[\begin{array}{ccc}
Q_{11} & Q_{12} & 0 \\
Q_{12} & Q_{11} & 0 \\
0 & 0 & Q_{33}
\end{array}\right]=\frac{E}{1-v^{2}}\left[\begin{array}{ccc}
1 & v & 0 \\
v & 1 & 0 \\
0 & 0 & (1-v) / 2
\end{array}\right]
$$

with $E$ and $v$ being the Young's modulus and Poisson's ratio.

Next, we define the resultant forces and moments acting on a layer, as shown in Fig. A.3, 


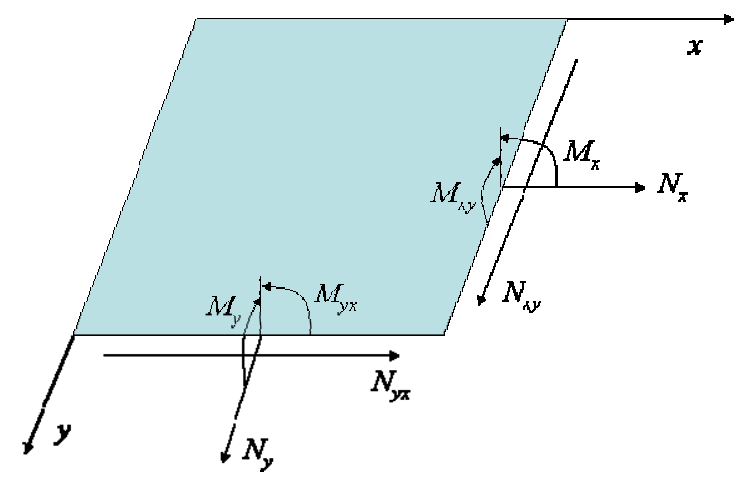

Fig. A.3 Positive sense of resultant forces and moments

$$
\left[\begin{array}{c}
N_{x} \\
N_{y} \\
N_{x y}
\end{array}\right]=\int_{-h / 2}^{h / 2}\left[\begin{array}{c}
\sigma_{x} \\
\sigma_{y} \\
\tau_{x y}
\end{array}\right] d z \quad, \quad\left[\begin{array}{c}
M_{x} \\
M_{y} \\
M_{x y}
\end{array}\right]=\int_{-h / 2}^{h / 2}\left[\begin{array}{c}
\sigma_{x} \\
\sigma_{y} \\
\tau_{x y}
\end{array}\right] z d z
$$

Substitution of (C.69) into (C.71) yields

$$
\begin{aligned}
& {\left[\begin{array}{l}
N_{x} \\
N_{y} \\
N_{x y}
\end{array}\right]=\mathbf{A}\left[\begin{array}{c}
\varepsilon_{x}^{0} \\
\varepsilon_{y}^{0} \\
\gamma_{x y}^{0}
\end{array}\right]+\mathbf{B}\left[\begin{array}{c}
\kappa_{x} \\
\kappa_{y} \\
\kappa_{x y}
\end{array}\right]-\left[\begin{array}{c}
N_{0} \\
N_{0} \\
0
\end{array}\right]} \\
& {\left[\begin{array}{c}
M_{x} \\
M_{y} \\
M_{x y}
\end{array}\right]=\mathbf{B}\left[\begin{array}{c}
\varepsilon_{x}^{0} \\
\varepsilon_{y}^{0} \\
\gamma_{x y}^{0}
\end{array}\right]+\mathbf{D}\left[\begin{array}{c}
\kappa_{x} \\
\kappa_{y} \\
\kappa_{x y}
\end{array}\right]-\left[\begin{array}{c}
M_{0} \\
M_{0} \\
0
\end{array}\right]}
\end{aligned}
$$

where

$$
\begin{aligned}
& \mathbf{A}=\sum_{k=1}^{n} \int_{h_{k}}^{h_{k+1}} \mathbf{Q} d z, \mathbf{B}=\sum_{k=1}^{n} \int_{h_{k}}^{h_{k+1}} \mathbf{Q} z d z, \mathbf{D}=\sum_{k=1}^{n} \int_{h_{k}}^{h_{k+1}} \mathbf{Q} z^{2} d z \\
& N_{0}=\sum_{k=1}^{n} \int_{h_{k}}^{h_{k+1}} \frac{\alpha \Delta T E}{1-v} d z, \quad M_{0}=\sum_{k=1}^{n} \int_{h_{k}}^{h_{k+1}} \frac{\alpha \Delta T E}{1-v} z d z
\end{aligned}
$$

Note that for functionally graded materials, both the elastic constant matrix $\mathbf{Q}$ and the CTE $\alpha$ are functions of the coordinate $z$. Furthermore, if the temperature distribution is not uniform across the thickness, $\Delta T$ is also a function of $z$.

Now, consider the equilibrium of the infinitesimal element in the laminate. The fact that the total forces and total moment acting on the element must add up to zero gives us the following equations of equilibrium

$$
\frac{\partial N_{x}}{\partial x}+\frac{\partial N_{x y}}{\partial y}=0 \quad, \frac{\partial N_{y}}{\partial y}+\frac{\partial N_{x y}}{\partial x}=0
$$




$$
\frac{\partial^{4} w_{0}}{\partial x^{4}}+2 \frac{\partial^{4} w_{0}}{\partial x^{2} \partial y^{2}}+\frac{\partial^{4} w_{0}}{\partial y^{4}}=-\left(q+N_{x} \frac{\partial^{2} w_{0}}{\partial x^{2}}+N_{y} \frac{\partial^{2} w_{0}}{\partial y^{2}}+2 N_{x y} \frac{\partial^{2} w_{0}}{\partial x \partial y}\right)
$$

where $q$ is the transverse loading.

Several types of boundary conditions can be prescribed for the above governing equations. For example, at the boundary $x=a$, one may have the following boundary conditions.

\section{Free Edge}

For a free edge, the bending moment and the shear force must vanish. Thus

$$
M_{x}(a, y)=0, \frac{\partial M_{x}}{\partial x}+2 \frac{\partial M_{x y}}{\partial y}=0
$$

Simply Supported Edge

For a simply supported edge, the deflection and the moment are zero. Thus,

$$
w_{0}(a, y)=0, M_{x}(a, y)=0
$$

\section{Rigidly Clamped Edge}

In this case, the deflection and the rotation are zero. Thus,

$$
w_{0}(a, y)=0,\left.\frac{\partial w_{0}(x, y)}{\partial x}\right|_{x=a}=0, \quad \varepsilon_{x}^{0}(a, y)=0
$$

\section{Elastically Clamped Edge}

In this case, the deflection is zero. But, the shafting and rotation are allowed due to the elasticity of the clamp. Thus,

$$
w_{0}(a, y)=0, N_{x}(a, y)=-E_{s} h \varepsilon_{x}^{0}(a, y), M_{x}(a, y)=\left.E_{s} h^{2} \frac{\partial w_{0}(x, y)}{\partial x}\right|_{x=a}
$$

where $E_{s}$ is the modulus of the clamp and $h$ is the total thickness of the plate. The products $E_{s} h$ and $E_{s} h^{2}$ can be viewed as the extensional and rotational stiffness of the clamp. When $C \rightarrow \infty$, (C.81) reduces to (C.80), the rigidly clamped case. 


\section{Appendix B: Thermal Shock Induced Microcrack Initiation}

We consider a body of infinite extent. The thermal elastic properties of the body are defined as follows:

$$
\begin{aligned}
& E=\text { Elastic Young's modulus } \\
& v=\text { Poisson's ratio } \\
& k=\text { Thermal conductivity } \\
& \kappa=\text { Thermal diffusivity } \\
& \alpha=\text { Coefficient of linear thermal expansion } \\
& \rho=\text { Mass density } \\
& c=\text { Specific heat capacity }
\end{aligned}
$$

If heat is liberated at the rate $q_{0}$ per unit time at the point $\mathbf{x}_{1}$, the temperature at $\mathbf{x}$ at time $t$ is

$$
T_{0}\left(\mathbf{x}, \mathbf{x}_{1}, t\right)=\frac{q_{0}}{4 \pi \rho c \kappa r} \operatorname{erfc}\left(\frac{r}{\sqrt{4 \kappa t}}\right)
$$

where $\operatorname{erfc}(x)$ is the complimentary error function, and

$$
r=\left|\mathbf{x}-\mathbf{x}_{1}\right|=\sqrt{r^{2}+r_{1}^{2}-2 r r_{1} \cos \varphi}
$$

Now consider the case when heat is librated at the rate $q \rho c$ over the entire body with the spatial distribution

$$
f(\mathbf{r})=\frac{q}{\rho c\left(\sqrt{\pi} r_{0}\right)^{3}} \exp \left(-\frac{r^{2}}{r_{0}^{2}}\right)
$$

The corresponding temperature distribution can be obtained by integrating (B.1),

$$
T(\mathbf{x}, t)=\frac{q}{\rho c\left(\sqrt{\pi} r_{0}\right)^{3}} \iiint_{\infty} \frac{1}{4 \pi \kappa\left|\mathbf{x}-\mathbf{x}_{1}\right|} \operatorname{erfc}\left(\frac{\left|\mathbf{x}-\mathbf{x}_{1}\right|}{\sqrt{4 \kappa t}}\right) \exp \left(-\frac{\left|\mathbf{x}_{1}\right|^{2}}{r_{0}^{2}}\right) d \mathbf{x}_{1}
$$

To carry out the integral, consider 


$$
\begin{aligned}
\frac{\partial T(\mathbf{x}, t)}{\partial t} & =\frac{q}{8 \rho c\left(\pi r_{0} \sqrt{\kappa t}\right)^{3}} \iiint_{\infty} \exp \left(-\frac{\left|\mathbf{x}_{1}\right|^{2}}{r_{0}^{2}}-\frac{\left|\mathbf{x}-\mathbf{x}_{1}\right|^{2}}{4 \kappa t}\right) d \mathbf{x}_{1} \\
& =\frac{q}{8 \rho c\left(\pi r_{0} \sqrt{\kappa t}\right)^{3}} \int_{0}^{\infty} \exp \left(-\frac{r_{1}^{2}}{r_{0}^{2}}\right) r_{1}^{2}\left[\int_{0}^{\pi} \exp \left(-\frac{r^{2}+r_{1}^{2}-2 r r_{1} \cos \varphi}{4 \kappa t}\right) \sin \varphi d \varphi\right] d r_{1} \\
& =\frac{q}{2 \pi^{2} r r_{0}^{3} \rho c \sqrt{\kappa t}} \int_{0}^{\infty}\left[\exp \left(\frac{r r_{1}}{\kappa t}\right)-1\right] \exp \left(-\frac{r_{1}^{2}}{r_{0}^{2}}-\frac{\left(r-r_{1}\right)^{2}}{4 \kappa t}\right) r_{1} d r_{1}
\end{aligned}
$$

This integral can be carried out to yield the rate of temperature change

$$
\dot{T}=\frac{\partial T(\mathbf{x}, t)}{\partial t}=\frac{q}{\rho c \pi^{3 / 2}\left(r_{0}^{2}+4 \kappa t\right)^{3 / 2}} \exp \left(\frac{-r^{2}}{r_{0}^{2}+4 \kappa t}\right)
$$

It can be shown that the maximum rate of temperature change occur at $t=0$ and $r=0$,

$$
\dot{T}_{\text {max }} \equiv \max \left\{\frac{\partial T(\mathbf{x}, t)}{\partial t}\right\}=\frac{q}{\rho c \pi^{3 / 2} r_{0}^{3}}
$$

The temperature is obtained by integrating (B.6),

$$
T(r, t)=\frac{q}{4 \pi r \rho c \kappa}\left[\operatorname{erf}\left(\frac{r}{r_{0}}\right)-\operatorname{erf}\left(\frac{r}{\sqrt{r_{0}^{2}+4 \kappa t}}\right)\right]+T_{0}
$$

where $T_{0}$ is the initial (uniform) temperature of the body, and $\operatorname{erf}(x)$ is the error function.

In the spherical coordinate system $(r, \theta, \varphi)$, the non-zero stresses induced by a spherically symmetric temperature field $T(r, t)$ are given by

$$
\begin{aligned}
& \sigma_{r}(r, t)=-\frac{2 \alpha E}{1-v} \frac{1}{r^{3}} \int_{0}^{r} T(s, t) s^{2} d s \\
& \sigma_{\theta}(r, t)=\sigma_{\varphi}(r, t)=\frac{\alpha E}{1-v}\left[\frac{1}{r^{3}} \int_{0}^{r} T(s, t) s^{2} d s-T(r, t)\right]
\end{aligned}
$$

Substitution of (B.8) into (B.9) - (B.10) yields

$$
\begin{aligned}
& \sigma_{r}(r)=\frac{-q \alpha E}{8 \pi^{3 / 2}(1-v) r^{3} \rho c \kappa}\left[2 r r_{0} e^{-r^{2} / r_{0}^{2}}+\sqrt{\pi}\left(2 r^{2}-r_{0}^{2}\right) \operatorname{erf}\left(\frac{r}{r_{0}}\right)\right] \\
& \sigma_{\theta}(r)=\sigma_{\varphi}(r)=\frac{q \alpha E}{18 \pi^{3 / 2}(1-v) r^{3} \rho c \kappa}\left[-2 r r_{0} e^{-r^{2} / r_{0}^{2}}+\sqrt{\pi}\left(2 r^{2}+r_{0}^{2}\right) \operatorname{erf}\left(\frac{r}{r_{0}}\right)\right]
\end{aligned}
$$


It can be easily shown that the stresses are a maximum at the center of the heating zone $(r=0)$, and decay rapidly away from it. The maximum values are

$$
\sigma_{r}(0)=\sigma_{\theta}(0)=\sigma_{\varphi}(0)=\frac{-q \alpha E}{3 \pi^{3 / 2}(1-v) \rho c \kappa r_{0}}
$$

The corresponding mechanical strain (total strain minus the thermal strain) components can be computed from the Hooke's law

$$
\varepsilon_{r}(0)=\varepsilon_{\theta}(0)=\varepsilon_{\varphi}(0)=\frac{-q \alpha(1-2 v)}{3 \kappa(1-v) \pi^{3 / 2} r_{0}}
$$

The strain energy density near the heating center can then be computed from (B.13) (B.14),

$$
U_{b}=\frac{q^{2} \alpha^{2} E(1-2 v)}{6 \rho^{2} c^{2} \kappa^{2}(1-v)^{2} \pi^{3} r_{0}^{2}}
$$

Next, consider that, under this thermal shock, microcracks are formed near the heated center. Let the number of microcracks per unite volume be $N$. For simplicity, we assume all the microcracks are penny-shaped cracks with radius $b$. Due to the microcracks, the effective modulus of the material near the heating center changes. If all the microcracks are randomly oriented, the effective Young's modulus of the cracked body can be approximated by

$$
E=E_{0}\left[1+\frac{16\left(1-v^{2}\right) N b^{3}}{9(1-2 v)}\right]^{-1}
$$

where $E_{0}$ is the Young's modulus of the un-cracked body.

The creation of microcracks reduces the amount of strain energy in the body. The amount of strain energy reduction should equal to the energy associated with all microcracks. Assuming the material in consideration is very brittle (no plastic deformation) and heat generation due to cracking is negligible, then the only energy associated with the creation of microcracks is the surface energy of the newly created crack surfaces,

$$
U_{s}=2 \pi N b^{2} \gamma
$$

where $\gamma$ is the surface energy per unit area of the material, which is related to fracture toughness $G_{c}$ through

$$
\gamma=\frac{1}{2} G_{c}=\frac{1-v^{2}}{2 E} K_{I c}^{2}
$$

The Griffith fracture criterion then requires

$$
\frac{d\left(U_{s}+U_{b}\right)}{d a}=0
$$

This yields 


$$
q=\frac{3 \pi^{2} k r_{0}}{2 \alpha}\left[1+\frac{16\left(1-v^{2}\right) N b^{3}}{9(1-2 v)}\right] \sqrt{\frac{2 \gamma \pi(1-v)}{E_{0} b(1+v)}}
$$

or

$$
q=\frac{3 \pi^{2} k r_{0}}{2 \alpha}\left[1+\frac{16\left(1-v^{2}\right) N b^{3}}{9(1-2 v)}\right] \sqrt{\frac{G_{c} \pi(1-v)}{E_{0} b(1+v)}}
$$




\section{Appendix C: Expressions of $\boldsymbol{Y}$ for Various Crack Configurations}

In the expressions of $Y$ for various cracks, the following denotations are used.

$$
\begin{aligned}
& Q=\frac{N_{0}\left(B_{11}+B_{12}\right)-M_{0}\left(A_{11}+A_{12}\right)}{\left(B_{11}+B_{12}\right)^{2}-\left(D_{11}+D_{12}\right)\left(A_{11}+A_{12}\right)} \\
& Q_{1}=\frac{\left(A_{11}+A_{12}\right)}{\left(B_{11}+B_{12}\right)^{2}-\left(D_{11}+D_{12}\right)\left(A_{11}+A_{12}\right)} \\
& A_{11}=\sum_{k=1}^{3} \int_{t_{k}}^{t_{k+1}} \frac{E_{k}}{1-v_{k}^{2}} d z \quad, \quad B_{11}=\sum_{k=1}^{3} \int_{t_{k}}^{t_{k+1}} \frac{E_{k}}{1-v_{k}^{2}} z d z \quad, \quad D_{11}=\sum_{k=1}^{3} \int_{t_{k}}^{t_{k+1}} \frac{E_{k}}{1-v_{k}^{2}} z^{2} d z \\
& A_{12}=\sum_{k=1}^{3} \int_{t_{k}}^{t_{k+1}} \frac{E_{k} v_{k}}{1-v_{k}^{2}} d z \quad, \quad B_{12}=\sum_{k=1}^{3} \int_{t_{k}}^{t_{k+1}} \frac{E_{k} v_{k}}{1-v_{k}^{2}} z d z \quad, \quad D_{12}=\sum_{k=1}^{3} \int_{t_{k}}^{t_{k+1}} \frac{E_{k} v_{k}}{1-v_{k}^{2}} z^{2} d z \\
& M_{0}=\sum_{k=1}^{3} \int_{t_{k}}^{t_{k+1}} \frac{\alpha_{k} E_{k}}{1-v_{k}} z d z, \quad N_{0}=\sum_{k=1}^{3} \int_{t_{k}}^{t_{k+1}} \frac{\alpha_{k} E_{k}}{1-v_{k}} d z \\
& t_{1}=-t_{4}=-\frac{h_{1}+h_{2}+h_{3}}{2}, t_{2}=\frac{h_{1}-h_{2}-h_{3}}{2}, t_{3}=\frac{h_{1}+h_{2}-h_{3}}{2} \\
& \Delta \alpha_{i}=\alpha_{i}-\bar{\alpha}_{i} \\
& \bar{\alpha}_{i}=\frac{\sum_{j \neq i} \alpha_{j} E_{j} h_{j}}{\sum_{j \neq i} E_{j} h_{j}} \\
& E_{a e}=\frac{h_{1}}{h_{a e}} E_{1}+\frac{h_{2}}{h_{a e}} E_{2} \\
& v_{a e}=\frac{h_{1}}{h_{a e}} v_{1}+\frac{h_{2}}{h_{a e}} v_{2} \\
& h_{a e}=h_{1}+h_{2} \\
& E_{c e}=\frac{h_{2}}{h_{c e}} E_{1}+\frac{h_{3}}{h_{c e}} E_{2} \\
& v_{c e}=\frac{h_{2}}{h_{c e}} v_{1}+\frac{h_{3}}{h_{c e}} v_{2} \\
& h_{c e}=h_{2}+h_{3} \\
& F_{1}=\frac{d_{2}^{2}}{\Gamma}+\frac{C_{3}^{2} d_{1}^{2}}{I}+12 C_{3} d_{1} d_{2} d_{3} \\
& F_{2}=\frac{C_{2}^{2}}{\Gamma}+\frac{C_{3}^{2}}{I}+12 C_{2} C_{3} d_{3} \\
& \lambda=\frac{1+\alpha}{1-\alpha} \\
& \delta=\frac{1+2 \lambda \eta+\lambda \eta^{2}}{2 \eta(1+\lambda \eta)}
\end{aligned}
$$




$$
\begin{aligned}
& A_{0}=\frac{1}{\eta}+\lambda \\
& I_{0}=\frac{1}{3}\left\{\lambda\left[3\left(\delta-\frac{1}{\eta}\right)^{2}-3\left(\delta-\frac{1}{\eta}\right)+1\right]+3 \frac{\delta}{\eta}\left(\delta-\frac{1}{\eta}\right)+\frac{1}{\eta^{3}}\right\} \\
& C_{1}=\frac{\lambda}{A_{0}} \\
& C_{2}=\frac{\lambda}{I_{0}}\left(\frac{1}{\eta}-\delta+\frac{1}{2}\right) \\
& C_{3}=\frac{\lambda}{12 I_{0}} \\
& d_{1}=\frac{1}{\eta}-\delta+\frac{1}{2} \\
& d_{2}=1-C_{1}-C_{2} d_{1} \\
& d_{3}=\lambda \eta^{2}(1+\eta) \\
& \Gamma=\frac{1}{1+\lambda\left(4 \eta+6 \eta^{2}+3 \eta^{3}\right)} \\
& I=\frac{1}{12\left(1+\lambda \eta^{3}\right)}
\end{aligned}
$$

\section{Crack A:}

$$
Y=\left(\frac{h_{2}^{3} E_{2}}{16 H^{2} E_{3}}\right)^{1 / 2}\left[\left(\frac{\Delta \alpha}{Q\left(1-v_{3}\right)}\right)^{2}+\left(t_{4}-\frac{a}{2}\right)^{2}\right]^{-1 / 2}
$$

where

$$
H=1.1215 \sqrt{h_{3}} \sqrt{\pi}\left(a / h_{3}\right)^{1 / 2}\left(1-a / h_{3}\right)^{1 / 2-s}\left(1+\lambda a / h_{3}\right)
$$

Crack $C$ :

$$
Y=\left(\frac{h_{2}^{3} E_{2}}{16}\right)^{1 / 2}\left(\frac{c_{3} F_{2}}{16 h_{3}^{3}} Q_{1}^{-2}+\frac{4 h_{3}\left(\Delta \alpha_{2}\right)^{2} F_{1}}{c_{3}} Q^{-2}\right)^{-1 / 2}
$$

where

$$
\begin{aligned}
& \eta=\frac{h_{3}}{h_{a e}} \\
& c_{3}=\frac{\kappa_{3}+1}{\mu_{3}} \\
& \alpha=\frac{\mu_{3}\left(\kappa_{a e}+1\right)-\mu_{a e}\left(\kappa_{3}+1\right)}{\mu_{3}\left(\kappa_{a e}+1\right)+\mu_{a e}\left(\kappa_{3}+1\right)}
\end{aligned}
$$


Crack D:

$$
Y=\left(\frac{h_{2}^{3} E_{2}}{16 h_{2}}\right)^{1 / 2}\left(\frac{c_{c e} F_{2}}{16 h_{c e}^{3}} Q_{1}^{-2} \rho^{2}+\frac{4 h_{c e}(\Delta \alpha)^{2} F_{1}}{c_{c e}} Q^{-2}\right)^{-1 / 2}
$$

where

$$
\begin{aligned}
& \eta=\frac{h_{c e}}{h_{3}} \\
& C_{c e}=\frac{\kappa_{c e}+1}{\mu_{c e}} \\
& \alpha=\frac{\mu_{c e}\left(\kappa_{1}+1\right)-\mu_{1}\left(\kappa_{c e}+1\right)}{\mu_{c e}\left(\kappa_{1}+1\right)+\mu_{1}\left(\kappa_{c e}+1\right)}
\end{aligned}
$$

Crack E:

$$
Y=\left(\frac{h_{2}^{3}}{16 \pi a E_{2}}\right)^{1 / 2}\left[\left(\frac{\Delta \alpha}{Q\left(1-v_{2}\right)}\right)^{2}+\left(\frac{h_{1}-h_{3}}{2}\right)^{2}\right]^{-1 / 2}
$$

Crack F:

$$
Y=\frac{Q h_{2} \sqrt{h_{2} E_{2}}}{4 \Delta \alpha} \sqrt{\left(\frac{1}{P_{1}}+\frac{P_{1} P_{2}^{2}}{G_{c}}\right)}
$$




\section{Appendix D: Schuster-Schwartzchild Two-Flux Approximation}

Theory:

The Schuster-Schwartzchild two-flux method provides an accurate description of one-dimensional radiation heat transfer in optically thin medium which is non-scattering or isotropically scattering. The model assumes the radiative intensity to be isotropic, but different over the upper and lower hemisphere, that is

$$
\mathrm{I}(\tau, \mu)=\left\{\begin{array}{c}
\mathrm{I}^{+}(\tau), 0<\theta<\pi / 2 \\
\mathrm{I}^{-}(\tau), \quad \pi / 2<\theta<\pi
\end{array}\right.
$$

Based on the above assumptions, the radiative heat flux $q_{r}$ reduces to the form

$$
q_{r}=C_{1} e^{2 \sqrt{1-\omega}}+C_{2} e^{-2 \sqrt{1-\omega}}
$$

where $\omega$ is the scattering albedo and $C_{1}$ and $C_{2}$ are constants obtained from the boundary conditions. The divergence of the radiative flux thus can be obtained and coupled to the energy equation through user-defined functions in Fluent.

\section{Validation of Two-Flux Approximation Scheme:}

Figure D.1 shows the boundary conditions on a rectangular enclosure used for validating the two-flux appoximation. For a non-scattering fluid $(\omega=0)$ enclosed in the long rectangular cavity modeled as a one-dimensional infinitely long slab with nonreflective walls $(\rho=0, \varepsilon=1)$, equation A2 reduces to the form

$$
q_{r}(z)=C_{1} e^{2 \beta z}+C_{2} e^{-2 \beta z}
$$

where $\beta$ is the absorption coefficient of the fluid with the constants $C_{1}$ and $C_{2}$ given by

$$
\begin{aligned}
& C_{1}=-\sigma\left(T_{\text {top }}^{4}-T^{4}\right) e^{-2 \beta L} \\
& C_{2}=\sigma\left(T_{\text {bottom }}^{4}-T^{4}\right)
\end{aligned}
$$

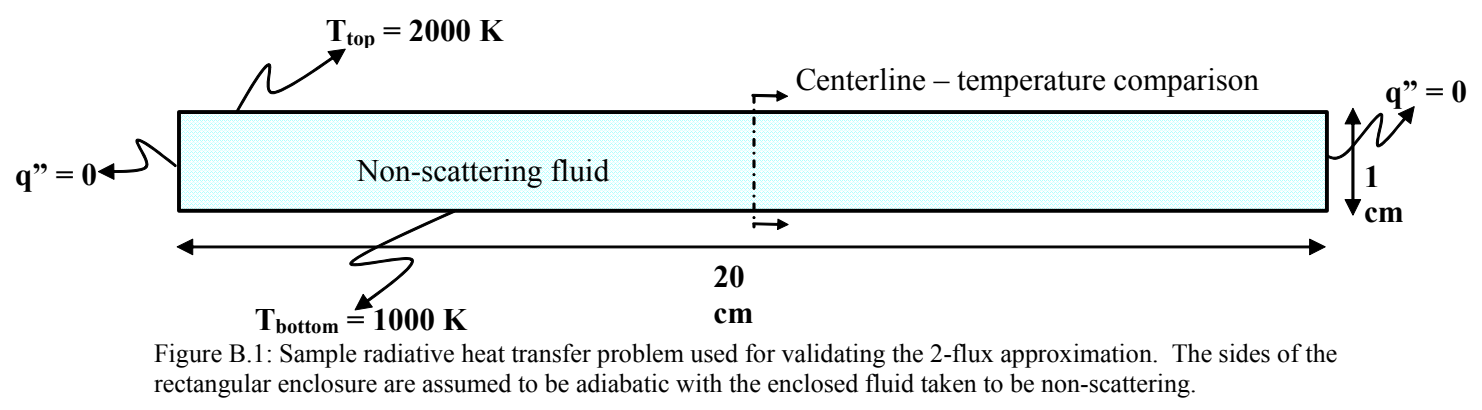


In equation D.4, T, $L$ and $\sigma$ refer to the temperature of the fluid, thickness of the enclosure and Stefan-Boltzman constant respectively.

Figure D.2 shows the temperature variation across the thickness of the enclosure along a line passing through the center and compares the results obtained from those using the Discrete Ordinates (DO) model in FLUENT. While good agreement in the temperature predictions is obtained for optically thin materials, deviations between the 2flux scheme and DO model predictions are observed for larger optical thickness beyond 0.01 .
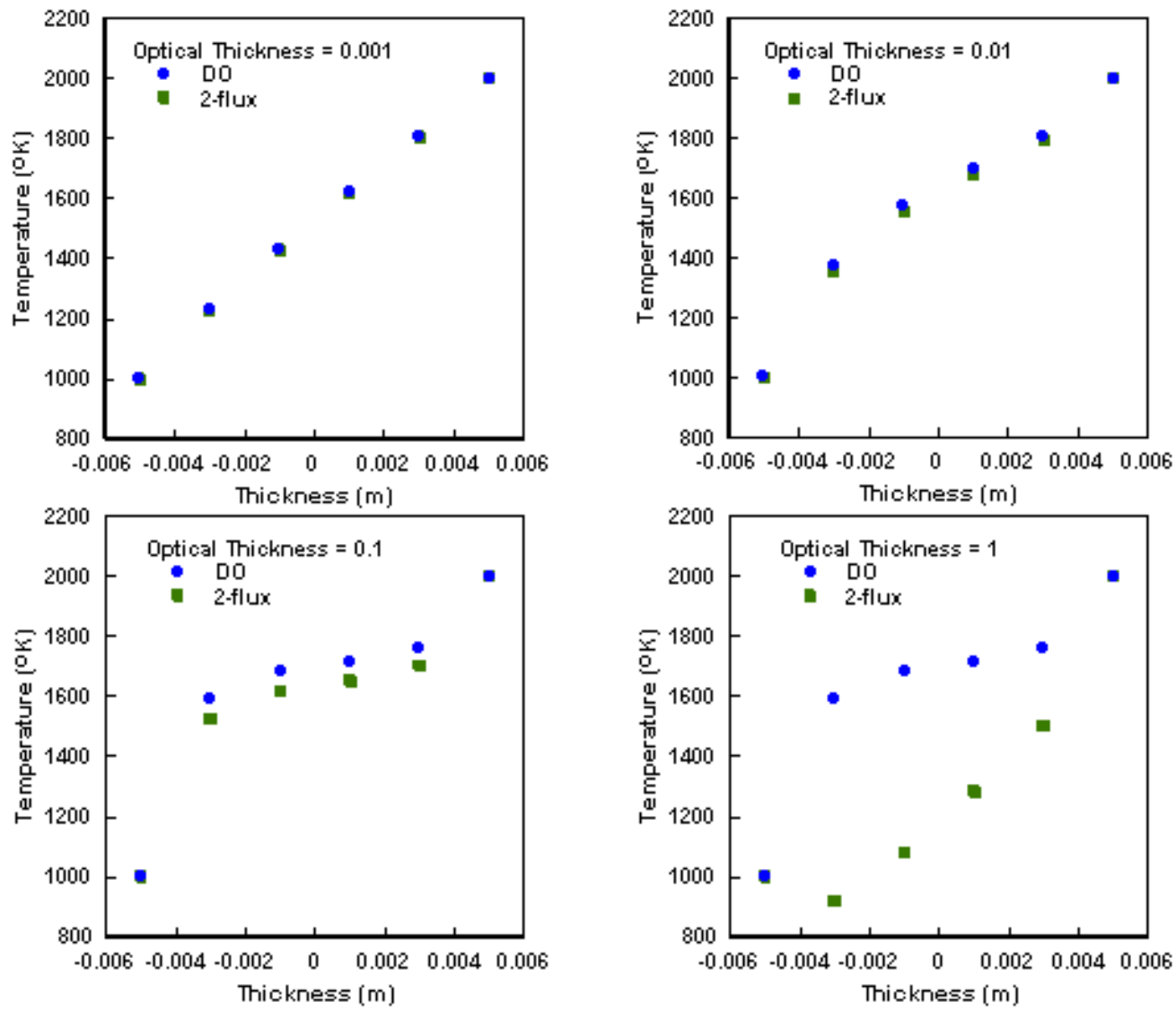

Figure B.2: Comparison of temperature variation along the centerline of the rectangular enclosure using an udf implementing 2-flux scheme with those obtained through Fluent's Discrete Ordinates (DO) model.

\section{Implementation of Two-Flux Approximation Scheme:}

Using Wien's law, it can be shown that $80 \%$ of fractional emissive power is contained within $1.4 \mu \mathrm{m}<\lambda<6.1 \mu \mathrm{m}$ for a temperature of $1000 \mathrm{~K}$. From Fig. D.4, it is seen that the transmittance of YSZ is independent of wavelength in the region of interest $(1.4 \mu \mathrm{m}<\lambda<6.1 \mu \mathrm{m})$. The absorption coefficient, assuming a non-scattering medium, is found to be $496.92 \mathrm{~m}^{-1}$. In the monolith type fuel cell model, the electrolyte thickness is $500 \mu \mathrm{m}$ or smaller. Subsequently, the optical thickness of the electrolyte, computed as the product of absorption coefficient times the material thickness, turns out to be 0.25 or smaller. Thus, radiation heat transfer in the electrolyte can be approximately modeled 
using the two-flux approximation method by assuming the electrolyte to be optically thin. Radiation is assumed to exist only along the ' $\mathrm{Y}$ ' direction and is neglected along the other two directions. These assumptions reduce the problem to the one considered in Fig. D.2.

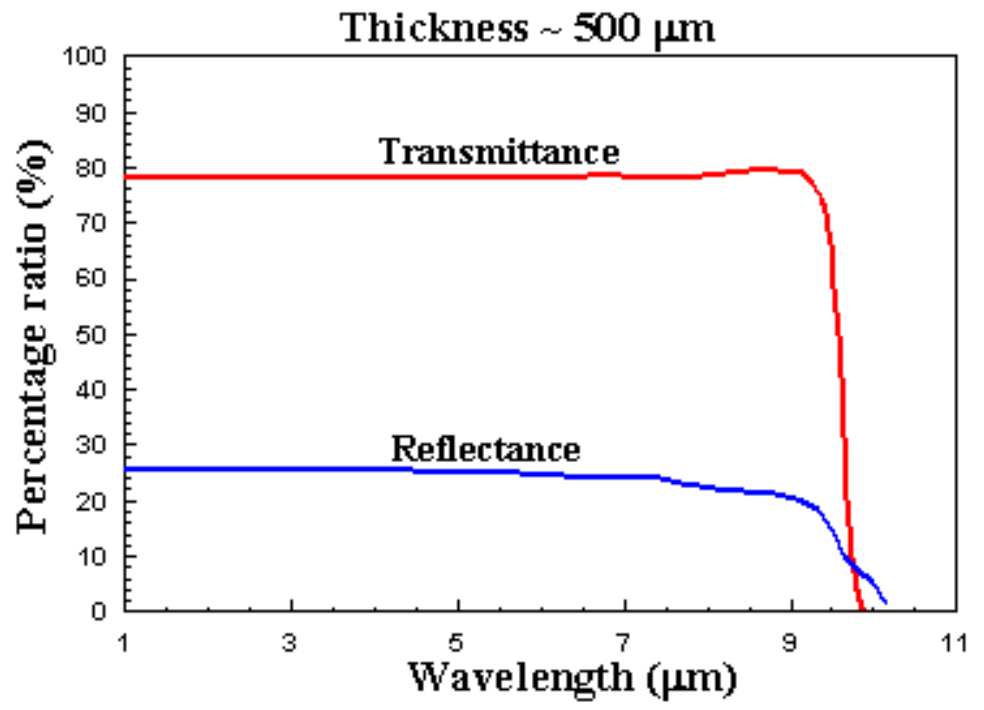

Figure D.4: Optical property of Yttria-Stabilized Zirconia (YSZ)

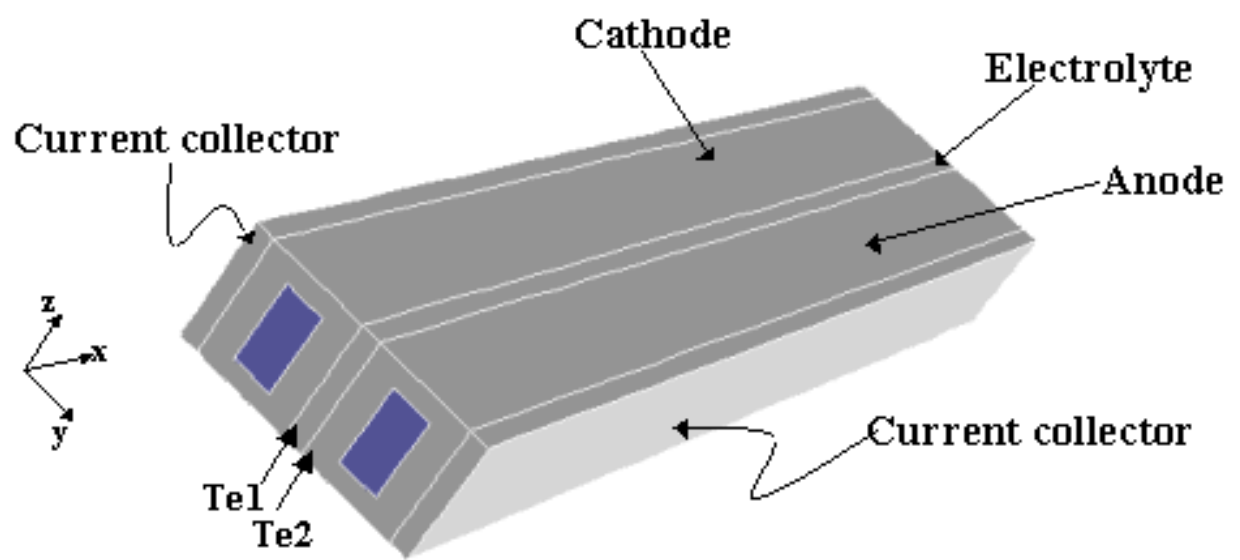

Figure D.5: Shows a schematic of a monolith type fuel cell with the 2-flux model implemented to account for radiation effects in the electrolyte. 


\section{Appendix E: 1D Derivation of Transient Solution}

The governing equation for the simplified 1-D, thermally-thin approximation, can be derived from conservation of energy on a control volume in the unit cell. Because the cell is thermally-thin and adiabatic, the control volume includes only the air in the airchannels as shown in Fig. 1. The temperature of the channel walls at any given point in the z-direction is equal to the air temperature at that point. Conservation of energy on this control volume requires that the rate of energy storage equal the rate of energy inflow minus outflow,

$$
\dot{E}_{\text {storage }}=\dot{E}_{\text {in }}-\dot{E}_{\text {out }}
$$

which is also written as,

$$
\rho c_{p} \frac{\delta T}{\delta t}=-\frac{\delta}{\delta \mathrm{z}}\left(-k \frac{\delta T}{\delta \mathrm{z}}+\rho c_{p} u T\right)
$$

or, more commonly,

$$
\frac{\delta T}{\delta t}+u \frac{\delta T}{\delta z}=\alpha \frac{\delta^{2} T}{\delta z^{2}}
$$

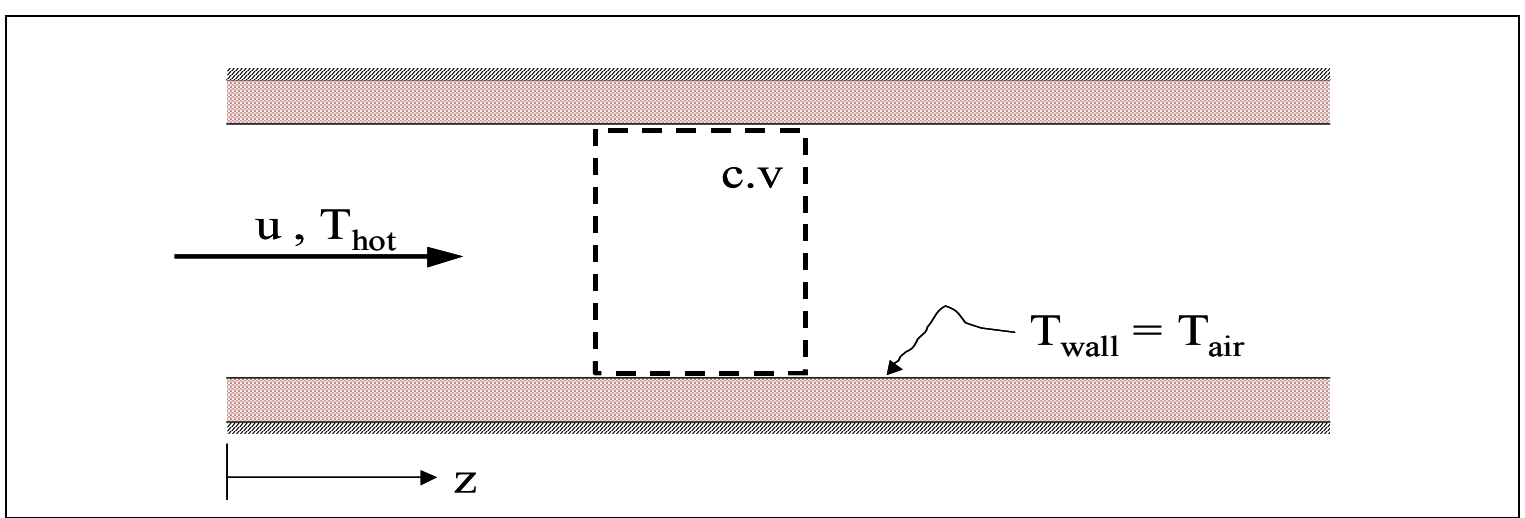

Figure 1. Schematic of air channel in unit cell shows 1-D control volume, assuming the channel walls are thermally thin and adiabatic.

A further simplifying assumption is to neglect heat diffusion in the air (right-hand side of equation E.3). Thus, the governing equation (E.3) reduces to,

$$
\frac{\delta T}{\delta t}+u \frac{\delta T}{\delta z}=0
$$




\section{Appendix F: 2D Transient Derivation}

We seek a solution of the governing equation,

$$
\frac{\delta T}{\delta t}+\frac{\delta T}{\delta z}=\frac{1}{P e} \frac{\delta^{2} T}{\delta z^{2}}
$$

subject to the boundary and initial conditions,

$$
\begin{aligned}
& T^{*}(0, t)-\sigma_{e f f} \frac{\partial T^{*}}{\partial z^{*}}(0, t)=F^{*}(t) \\
& \rightarrow T(0, t)-\sigma \frac{\partial T}{\partial z}(0, t)=F(t) \\
& \left.\frac{\delta T}{\delta z}\right|_{z=1}=0 \\
& T(z, 0)=1
\end{aligned}
$$

where, $\sigma=\sigma_{\text {eff }} / L$.

We expect the solution to take the form,

$$
T(z, t)=\theta(z, t) \cdot e^{A z+B t}
$$

Since $A$ and $B$ are arbitrary constants, they can be specified in such a manner that greatly simplifies the work,

$$
A=\frac{P e}{2} ; \quad B=-\frac{P e}{4}
$$

reducing the equation to,

$$
\frac{\delta \theta}{\delta t}=\frac{1}{P e} \frac{\delta^{2} \theta}{\delta \mathrm{z}^{2}}
$$

The boundary and initial conditions of the original problem are also transformed, 


$$
\begin{aligned}
& T(0, t)-\sigma \frac{\partial T}{\partial z}(0, t)=F(t)=\theta(0, t) \cdot e^{B t}-\sigma\left[\left.\frac{\delta \theta}{\delta z}\right|_{z=0}+\theta(0, t)\right] e^{B t} \\
& \rightarrow \theta(0, t)-\frac{\sigma}{1-\sigma} \frac{\delta \theta}{\delta z}(0, t)=\frac{1}{1-\sigma} F(t) \cdot e^{-B t}=f(t) \\
& \left.\frac{\delta T}{\delta z}\right|_{z=1}=0=\left.\frac{\delta \theta}{\delta z}\right|_{z=1} \cdot e^{A+B t}+\theta(1, t) \cdot A \cdot e^{A+B t} \\
& \rightarrow \frac{\delta \theta}{\delta z}(1, t)+A \theta(1, t)=0 \\
& T(z, 0)=1=\theta(z, 0) \cdot e^{A z} \\
& \rightarrow \theta(z, 0)=e^{-A z}=f^{*}(z)
\end{aligned}
$$

Next, assume

$$
\theta(z, t)=\theta^{*}(z, t)+D_{1}(t) \cdot z+D_{2}(t)
$$

and the boundary conditions yield the functions $D_{1}(t)$ and $D_{2}(t)$,

$$
\begin{aligned}
& \theta(0, t)-\frac{\sigma}{1-\sigma} \frac{\delta \theta}{\delta z}(0, t)=f(t)=\theta^{*}(0, t)+D_{2}(t)-\frac{\sigma}{1-\sigma} \frac{\delta \theta^{*}}{\delta z}(0, t)-\frac{\sigma}{1-\sigma} D_{1}(t) \\
& \text { if } D_{2}(t)-\frac{\sigma}{1-\sigma} D_{1}(t)=f(t) \text { then } \theta^{*}(0, t)-\frac{\sigma}{1-\sigma} \frac{\delta \theta^{*}}{\delta z}(0, t)=0 \\
& \frac{\delta \theta}{\delta z}(1, t)+A \theta(1, t)=0=\theta_{z}^{*}(1, t)+D_{1}(t)+A \theta^{*}(1, t)+A D_{1}(t)+A D_{2}(t) \\
& \text { if } \theta_{z}^{*}(1, t)+A \theta^{*}(1, t)=0 \text { then }[1+A] D_{1}(t)+A D_{2}(t)=0 \\
& \left.[1+A] D_{1}(t)=-A D_{2}(t)=-A f(t)-\frac{\sigma A}{1-\sigma} D_{1}(t)\right] \\
& \quad \rightarrow D_{1}(t)=\frac{-A(1-\sigma) f(t)}{1+A-\sigma} D_{2}(t)=\frac{(1-\sigma)(1+A)}{1+A-\sigma} f(t)
\end{aligned}
$$

Thus derivation becomes,

$$
\begin{gathered}
\theta(z, t)=\theta^{*}(z, t)+[1+A-A z] \frac{(1-\sigma)}{1+A-\sigma} f(t) \\
\theta(z, 0)=f^{*}(z)=\theta^{*}(z, 0)+[1+A-A z] \frac{(1-\sigma)}{1+A-\sigma} f(0) \\
\rightarrow \theta^{*}(z, 0)=f^{*}(z)-[1+A-A z] \frac{(1-\sigma)}{1+A-\sigma} f(0)
\end{gathered}
$$


Taking the derivatives of eqn and substituting yields,

$$
\begin{aligned}
& \frac{\delta \theta}{\delta t}=\frac{1}{\operatorname{Pe}} \frac{\delta^{2} \theta}{\delta z^{2}}=\theta_{t}^{*}(z, t)+[1+A-A z] \frac{(1-\sigma)}{1+A-\sigma} f^{\prime}(t)=\frac{1}{P e} \theta_{z z}^{*}(z, t) \\
& \rightarrow \theta_{t}^{*}(z, t)=\frac{1}{P e} \theta_{z z}^{*}(z, t)-S(z, t) \\
& \text { where } S(z, t)=[1+A-A z] \frac{(1-\sigma)}{1+A-\sigma} f^{\prime}(t)
\end{aligned}
$$

The homogenous portion of can be solved using separation of variables,

$$
\theta^{*}(z, t)=Z(z) \Psi(t)
$$

yielding the following ODE in space,

$$
Z^{\prime \prime}+\lambda^{2} P e Z=0
$$

which has a solution of the form,

$$
Z(z)=C_{1} \sin (\lambda \sqrt{P e} z)+C_{2} \cos (\lambda \sqrt{P e} z)
$$

subject to the boundary conditions,

$$
\begin{aligned}
& \theta^{*}(0, t)-\frac{\sigma}{1-\sigma} \frac{\delta \theta^{*}}{\delta z}(0, t)=0=\left[Z(0)-\frac{\sigma}{1-\sigma} Z^{\prime}(0)\right] \Psi(t) \rightarrow Z(0)-\frac{\sigma}{1-\sigma} Z^{\prime}(0)=0 \\
& \frac{\delta \theta^{*}}{\delta z}(L, t)+A \theta^{*}(L, t)=0=\Psi(t)\left[Z^{\prime}(L)+A \cdot Z(L)\right] \\
& \rightarrow Z^{\prime}(L)+A \cdot Z(L)=0
\end{aligned}
$$

The eigenvectors for this problem are,

$$
C_{1}\left[\sin \left(\lambda_{n} \sqrt{P e z}\right)+\frac{\sigma}{1-\sigma} \lambda_{n} \sqrt{P e} \cos \left(\lambda_{n} \sqrt{P e z}\right)\right] \quad n=1,2,3 \ldots
$$

where the eigenvalues are the solutions of the eigencondition,

$$
\left(\lambda_{n} \sqrt{P e}+\frac{A \sigma}{1-\sigma}\right) \cos \left(\lambda_{n} \sqrt{P e}\right)+\left(A-\frac{\sigma}{1-\sigma} \lambda_{n}^{2} P e\right) \sin \left(\lambda_{n} \sqrt{P e}\right)=0
$$

which must be solved numerically. 
Next, assume a solution of the form,

$$
\theta^{*}(z, t)=\sum_{n=1}^{\infty} C_{n}(t)\left[\sin \left(\lambda_{n} \sqrt{P e} z\right)+\frac{\sigma}{1-\sigma} \lambda_{n} \sqrt{P e} \cos \left(\lambda_{n} \sqrt{P e} z\right)\right]
$$

where,

$$
C_{n}(t)=\frac{\int_{0}^{1} \theta^{*}(z, t)\left[\sin \left(\lambda_{n} \sqrt{P e} z\right)+\frac{\sigma}{1-\sigma} \lambda_{n} \sqrt{P e} \cos \left(\lambda_{n} \sqrt{P e} z\right)\right] d z}{\int_{0}^{1}\left[\sin \left(\lambda_{n} \sqrt{P e z}\right)+\frac{\sigma}{1-\sigma} \lambda_{n} \sqrt{P e} \cos \left(\lambda_{n} \sqrt{P e} z\right)\right]^{2} d z}
$$

The denominator is the norm, which we will call $G_{n}$, thus,

$$
C_{n}(t)=\frac{1}{G_{n}} \int_{0}^{1} \theta^{*}(z, t)\left[\sin \left(\lambda_{n} \sqrt{P e z}\right)+\frac{\sigma}{1-\sigma} \lambda_{n} \sqrt{P e} \cos \left(\lambda_{n} \sqrt{P e z}\right)\right] d z
$$

The next step is to write an ODE for $C_{n}$,

$$
\frac{d C_{n}}{d t}=\frac{1}{G_{n}} \int_{0}^{1} \frac{d \theta^{*}}{d t}(z, t)\left[\sin \left(\lambda_{n} \sqrt{P e} z\right)+\frac{\sigma}{1-\sigma} \lambda_{n} \sqrt{P e} \cos \left(\lambda_{n} \sqrt{P e z}\right)\right] d z
$$

which, from eqn (C.21) can be rewritten as,

$$
\begin{aligned}
\frac{d C_{n}}{d t}= & \frac{1}{G_{n} P e} \int_{0}^{1} \frac{d^{2} \theta^{*}}{d z^{2}}(z, t)\left[\sin \left(\lambda_{n} \sqrt{P e} z\right)+\frac{\sigma}{1-\sigma} \lambda_{n} \sqrt{P e} \cos \left(\lambda_{n} \sqrt{P e} z\right)\right] d z \\
& -\frac{1}{G_{n}} \int_{0}^{1} S(z, t)\left[\sin \left(\lambda_{n} \sqrt{P e} z\right)+\frac{\sigma}{1-\sigma} \lambda_{n} \sqrt{P e} \cos \left(\lambda_{n} \sqrt{P e} z\right)\right] d z
\end{aligned}
$$

The first integral on the RHS is integrated by parts twice, and sparing the details, eqn (F.22) is conveniently rewritten as,

$$
\begin{aligned}
\frac{d C_{n}}{d t}= & -\frac{\lambda_{n}^{2}}{G_{n}} \int_{0}^{1} \theta^{*}(z, t)\left[\sin \left(\lambda_{n} \sqrt{P e} z\right)+\frac{\sigma}{1-\sigma} \lambda_{n} \sqrt{P e} \cos \left(\lambda_{n} \sqrt{P e} z\right)\right] d z \\
& -\frac{1}{G_{n}} \int_{0}^{1} S(z, t)\left[\sin \left(\lambda_{n} \sqrt{P e} z\right)+\frac{\sigma}{1-\sigma} \lambda_{n} \sqrt{P e} \cos \left(\lambda_{n} \sqrt{P e} z\right)\right] d z
\end{aligned}
$$

or,

$$
\frac{d C_{n}}{d t}+\alpha \lambda_{n}^{2} C_{n}=-\frac{1}{G_{n}} \int_{0}^{1} S(z, t)\left[\sin \left(\lambda_{n} \sqrt{P e z}\right)+\frac{\sigma}{1-\sigma} \lambda_{n} \sqrt{P e} \cos \left(\lambda_{n} \sqrt{P e} z\right)\right] d z
$$


for which the solutions is,

$$
\begin{aligned}
C_{n}(t)= & C_{n}(0) e^{-\lambda_{n}^{2} t} \\
& -e^{-\lambda_{n}^{2} t} \frac{1}{G_{n}} \int_{0}^{t} e^{\lambda_{n}^{2} \tau} \int_{0}^{1} S(z, \tau)\left[\sin \left(\lambda_{n} \sqrt{P e z}\right)+\frac{\sigma}{1-\sigma} \lambda_{n} \sqrt{P e} \cos \left(\lambda_{n} \sqrt{P e z}\right)\right] d z d \tau
\end{aligned}
$$

where,

$$
C_{n}(0)=\frac{1}{G_{n}} \int_{0}^{1} \theta^{*}(z, 0)\left[\sin \left(\lambda_{n} \sqrt{P e} z\right)+\frac{\sigma}{1-\sigma} \lambda_{n} \sqrt{P e} \cos \left(\lambda_{n} \sqrt{P e} z\right)\right] d z
$$

